
Destroy this report when no longer needed. Do not return it to the originator.

The findings in this report are not to be construed as an official

Department of the Army position unless so designated by other authorized documents.

The contents of this report are not to be used for advertising, publication, or promotional purposes. Citation of trade names does not constitute an official endorsement or approval of the use of such commercial products. 


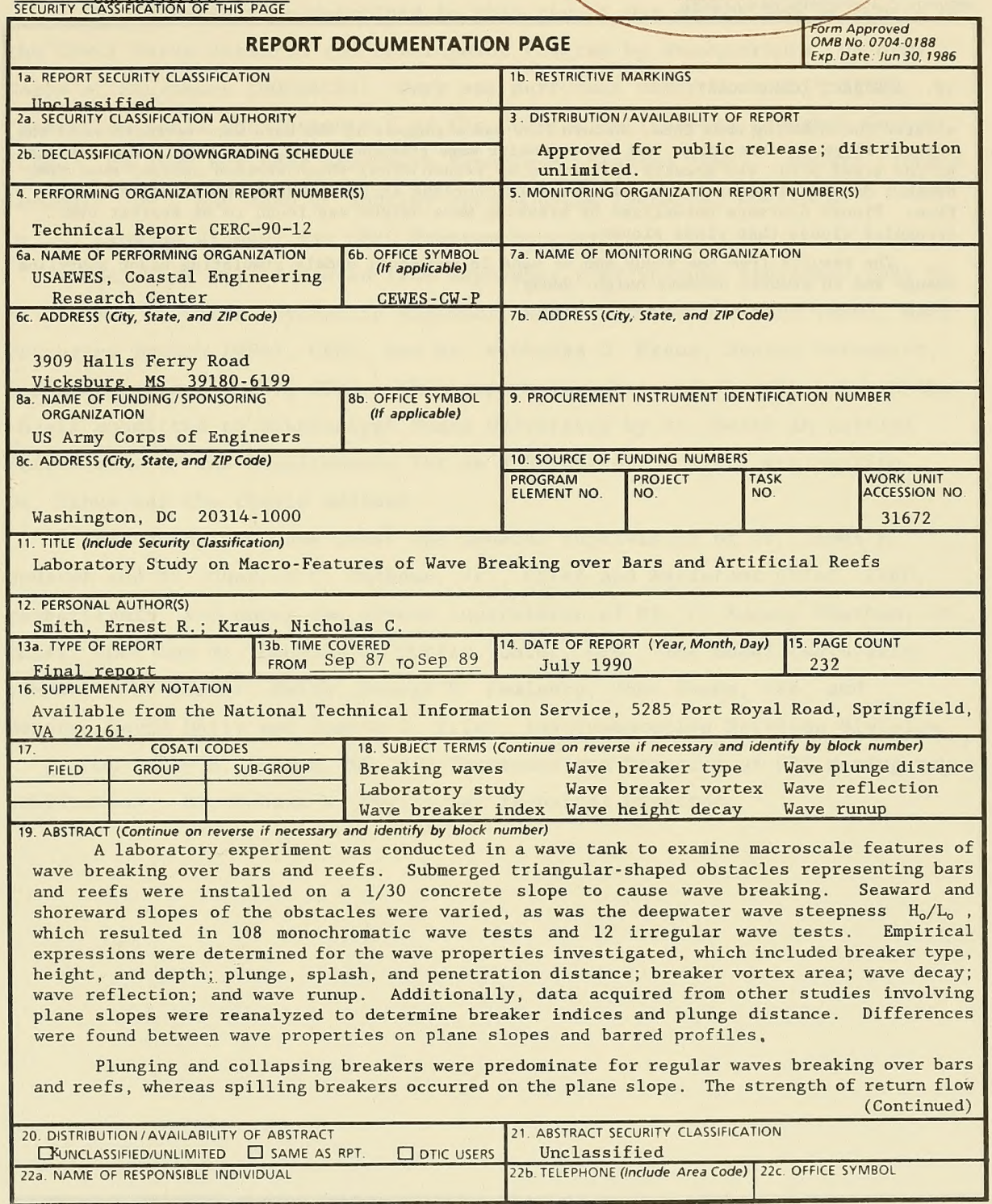




\section{ABSTRACT (Continued).}

altered the breaking wave form. Return flow was strongest if the bars were terraced or if the seaward slope $\beta_{1}$ was steep, and the deepwater wave steepness $\mathrm{H}_{\mathrm{o}} / \mathrm{L}_{\mathrm{o}}$ was small. The position of the break point was greatly influenced by return flow, which exerted control over the breaker depth. Breaker height was found to increase in the presence of strong return flow. Plunge distance normalized by breaking wave height was found to be shorter over irregular slopes than plane slopes.

The results from the study can be used in numerical models simulating beach shoreline change and in studies of wave height decay. 
The investigation described in this report was authorized as a part of the Civil Works Research and Development Program by Headquarters, US Army Corps of Engineers (HQUSACE). Work was performed under Work Unit 31672 , "Nearshore Waves and Currents," at the Coastal Engineering Research Center (CERC), US Army Engineer Waterways Experiment Station (WES). Messrs. John H. Lockhart, Jr., and John G. Housley were HQUSACE Technical Monitors.

Dr. C. Linwood Vincent was CERC Program Manager.

The study was conducted from September 1987 through September 1989 by Mr. Ernest R. Smith, Hydraulic Engineer, Wave Dynamics Division (WDD), Wave Processes Branch (WPB), CERC, and Dr. Nicholas C. Kraus, Senior Scientist, Research Division (RD), CERC. This report is substantially the same as the thesis submitted to Mississippi State University by Mr. Smith in partial fulfillment of the requirements for an M.S. degree in civil engineering. Dr. Kraus was the thesis advisor.

This study was done under the general supervision of Dr. James R. Houston and Mr. Charles C. Calhoun, Jr., Chief and Assistant Chief, CERC, respectively, and under the direct supervision of $\mathrm{Mr}$. C. Eugene Chatham, Jr., Chief, WDD; and Mr. Douglas G. Outlaw, Chief, WPB. The model tests were conducted by Messrs. Smith, George M. Kaminsky, John Evans, WPB, and Messrs. David Daily and Lonnie L. Friar, Instrumentation Services Division. COL Larry B. Fulton, EN, was Commander and Director of WES during report publication. Dr. Robert W. Whalin was Technical Director. 
LIST OF TABLES

LIST OF FIGURES

CONVERSION FACTORS, NON-SI TO SI (METRIC)

UNITS OF MEASUREMENTS . . . . . . . . . . . . . . . . . . . . . . . . . 8

PART I: INTRODUCTION . . . . . . . . . . . . . . . . . . . . . . . 9

Background . . . . . . . . . . . . . . . . . . 9

Purpose . . . . . . . . . . . . . . . . . . . . . . 10

Content . . . . . . . . . . . . . . . . . . . . . 11

PART II: REVIEW OF RELATED STUDIES . . . . . . . . . . . . . . . . . . . 13

Breaker Type . . . . . . . . . . . . . . . . . . . . . . . 13

Wave Reflection... . . . . . . . . . . . . . . . . . 17

Breaker Index. . . . . . . . . . . . . . . . . . . . . . . 19

Plunge Distance. . . . . . . . . . . . . . . . . . . . . . . 33

Breaker Vortex . . . . . . . . . . . . . . . . . . . . . 34

Wave Height Decay . . . . . . . . . . . . . . . . . . . . . . 35

Wave Runup . . . . . . . . . . . . . . . . . . . . . . 40

Analysis of Previous Data. . . . . . . . . . . . . . . . 42

Summary . . . . . . . . . . . . . . . . . . . . . . 58

PART III: EXPERIMENT ARRANGEMENT . . . . . . . . . . . . . . . . . . . . 59

Facility and Equipment . . . . . . . . . . . . . . . . . . 59

Design Wave Conditions . . . . . . . . . . . . . . . . . . 62

Bar Design . . . . . . . . . . . . . . . . . . . . . 66

Test Procedure . . . . . . . . . . . . . . . . . . . 70

PART IV: RESUlTS . . . . . . . . . . . . . . . . . . . . . . . . 75

Breaker Type . . . . . . . . . . . . . . . . . . . . 76

Wave Reflection... . . . . . . . . . . . . . . . . 82

Breaker Index . . . . . . . . . . . . . . . . . . . . . . . . 84

Plunge Distance. . . . . . . . . . . . . . . . . . . . . . . . . 99

Splash Distance. . . . . . . . . . . . . . . . . . . . . 101

Penetration Distance . . . . . . . . . . . . . . . . . . 104

Breaker Vortex . . . . . . . . . . . . . . . . . . . . . . . 106

Wave Height Decay . . . . . . . . . . . . . . . . . . . . . 109

Wave Runup . . . . . . . . . . . . . . . . . . . . . . . 109

Wave Steepness Scaling . . . . . . . . . . . . . . . 115

Irregular Waves . . . . . . . . . . . . . . . . . . . . 120

Measurement Errors . . . . . . . . . . . . . . . . . . . . . . . 141

PART V: SUMMARY AND RECOMMENDATONS . . . . . . . . . . . . . . . . . . . 144

Monochromatic Tests . . . . . . . . . . . . . . . . . . . . 144

Irregular Wave Tests . . . . . . . . . . . . . . . . . . . 147

Recommendations for Future Study . . . . . . . . . . . . . . . . . 147

REFERENCES . . . . . . . . . . . . . . . . . . . . . 149

APPENDIX A: BREAKER DATA . . . . . . . . . . . . . . . . . . . . . . . . A1 
Page

APPENDIX B: TRACINGS OF WAVE FORMS . . . . . . . . . . . . . . . . . . B1

APPENDIX C: WAVE HEIGHT DATA . . . . . . . . . . . . . . . . . C1

APPENDIX D: NOTATION . . . . . . . . . . . . . . . . . D1 


\section{LIST OF TABLES}

No.

$\underline{\text { Page }}$

1 Summary of Coefficients and Exponents for Breaker Depth Index . . . . . . . . . .

2 Summary of Coefficients and Exponents for

Breaker Height Index

28

Data Summary for Breaker Index and

Plunge Distance on Plane Slopes... . . . . . . . . . . . . 44

Maximum Wave Height Observed in the 18-In. Tank

Range of Wave Conditions . . . . . . . . . . . . . . . . . . . 64

Prototype and Model Conditions of Case CE400 (Pilot Test) . . . 72

List of Design Parameters for Base Tests . . . . . . . . . . . 72

List of Design Parameters for Base Test Variations . . . . . . 73

Irregular Wave Test Design Parameters . . . . . . . . . . . . . 74

Summary of Monochromatic Base Tests . . . . . . . . . . . . . . 77

Summary of Irregular Wave Tests.. . . . . . . . . . . . . . . 80

Summary of Scaled Base Tests . . . . . . . . . . . . . . 116

A1

Monochromatic Wave Base Tests . . . . . . . . . . . . . . . A2

Summary of Plane-Slope Tests . . . . . . . . . . . . . . . . . A7

A3

Results of Iversen (1952) . . . . . . . . . . . . . . . . . . A8

A4

A5

Results of Horikawa and Kuo (1967) . . . . . . . . . . . . . A10

Results of Galvin (1969) . . . . . . . . . . . . . . . . . . A13

A6

A7

Results of Saeki and Sasaki (1973) . . . . . . . . . . . . A13

Results of Iwagaki et al. (1974) . . . . . . . . . . . . . A14

Results of Walker (1974b) . . . . . . . . . . . . . . . . A15

A10

Results of Singamsetti and Wind (1980) . . . . . . . . . . . A16

Results of Mizuguchi (1981). . . . . . . . . . . . . . . . . . A19

A11

\section{LIST OF FIGURES}

Spilling wave

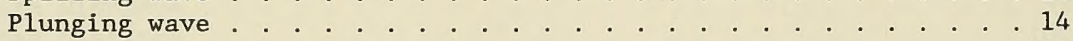

Collapsing wave . . . . . . . . . . . . . . . . . . . . . . 15

Definition sketch of wave at incipient breaking . . . . . . . . . 19

Breaker depth index predictions . . . . . . . . . . . . . . . . 26

Breaker height index predictions . . . . . . . . . . . . . . . . 29

Definition sketch of plunge distance and vortex area . . . . . . 33

Definition sketch of wave runup . . . . . . . . . . . . . . . . . 40

Breaker depth index as a function of $\mathrm{H}_{\mathrm{o}} / \mathrm{L}_{\mathrm{o}}$. . . . . . . . . . . 45

Function $\mathrm{a}(\mathrm{m})$. . . . . . . . . . . . . . . . . . 48

Function $\mathrm{b}(\mathrm{m})$. . . . . . . . . . . . . . . . . . . 48

Calculated values of $\gamma_{b}$ for plane slopes as a

function of $\mathrm{H}_{\mathrm{o}} / \mathrm{L}_{\mathrm{o}}$. . . . . . . . . . . . . . . . . . . . . . . 49

Breaker height index as a function of $\mathrm{H}_{\mathrm{o}} / \mathrm{L}_{\mathrm{o}}$. . . . . . . . . 50 
$\mathrm{X}_{\mathrm{p}} / \mathrm{H}_{\mathrm{b}}$ as a function of $\xi_{0}$. . . . . . . . . . . . . 55

$\mathrm{X}_{\mathrm{p}} / \mathrm{H}_{\mathrm{b}}$ as a function of $\xi_{\mathrm{b}}$................ . . . 57

$\mathrm{X}_{\mathrm{p}} / \mathrm{H}_{\mathrm{o}}$ as a function of $\xi_{0}$. . . . . . . . . . . . . . . . . 57

Sketch of tank used in study . . . . . . . . . . . . . . 59

Gages 3-6. . . . . . . . . . . . . . . . . . . . 60

Video equipment used in study . . . . . . . . . . . . . . 61 61

Definition sketch of typical bar . . . . . . . . . . . . . 62

Maximum $\mathrm{H}_{0} / \mathrm{L}_{\circ}$ as a function of $\mathrm{T}$. . . . . . . . . . . . 64

Predicted wave forms produced by a periodic wave

generator, after Galvin (1970) . . . . . . . . . . . . 65

Sketch of terraced bar . . . . . . . . . . . . . . . 67

Shape of typical bar used in study . . . . . . . . . . . . . 68

Spectral shape of irregular wave signal used to

control wave board and predicted shape of

water surface spectrum . . . . . . . . . . . . . . . . . . 74

Breaker type as a function of $\xi_{0}$. . . . . . . . . . . . . 80

Breaker type as a function of the relative

length parameter

Comparison of measured $\mathrm{K}_{\mathrm{r}}$ with predicted values of Battjes (1975) and Miche (1951) as a

function of $\xi_{0}$

Measured wave reflection and predicted values

of Ahrens (1987) as a function of $\mathrm{P}$. . . . . . . . . . 83

Wave reflection as a function of $\beta_{1}$. . . . . . . . . . . . . 84

$\gamma_{b}$ as a function of $\xi_{0}$. . . . . . . . . . . . . . . . 86

Collapsing wave at incipient breaking . . . . . . . . . . . 87

Expression of $\gamma_{b}$ as a function of $\xi_{0}$. . . . . . . . . . . . . 89

Exponential expression of $\gamma_{b}$ as a function

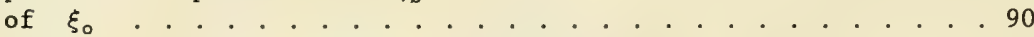

Comparison of breaker depth relationships developed for barred profiles and plane slopes . . . . . . . . 90

$\Omega_{\mathrm{b}}$ as a function of $\mathrm{H}_{\mathrm{o}} / \mathrm{L}_{\mathrm{o}}$. . . . . . . . . . . . . . . . . 91

Function $\mathrm{C}\left(\beta_{1}\right)$. . . . . . . . . . . . . . . 94

Function $\mathrm{n}\left(\beta_{1}\right)$. . . . . . . . . . . . . . . . . . 94

Regression curves of $\Omega_{b}$ as a function of $H_{o} / L_{0}$. . . . . . . . 95

Calculated values of $\Omega_{b}$ as a function of $H_{o} / L_{o}$. . . . . . . . 95

$\Omega_{b}$ as a function of $\mathrm{H}_{0} / \mathrm{L}_{0}$. . . . . . . . . . . . . . 98

$\mathrm{X}_{\mathrm{p}} / \mathrm{H}_{\mathrm{b}}$ as a function of $\xi_{0}$. . . . . . . . . . . . . . . . . 99

$\mathrm{X}_{\mathrm{p}} / \mathrm{H}_{\mathrm{b}}$ as a function of $\xi_{\mathrm{b}}$. . . . . . . . . . . . . . . . 100

$\mathrm{X}_{\mathrm{p}} / \mathrm{H}_{\mathrm{o}}$ as a function of $\xi_{0}$. . . . . . . . . . . . . . . 101

$\mathrm{X}_{\mathrm{s}} / \mathrm{H}_{\mathrm{b}}$ as a function of $\xi_{\mathrm{o}}$. . . . . . . . . . . . . . 102

$\mathrm{X}_{\mathrm{p}} / \mathrm{X}_{\mathrm{s}}$ as a function of $\xi_{0}$. . . . . . . . . . . . . . 103

$\mathrm{X}_{\mathrm{p}} / \mathrm{X}_{\mathrm{s}}$ as a function of $\xi_{\mathrm{b}}$. . . . . . . . . . . . . . . 103

$\mathrm{X}_{\mathrm{t}} / \mathrm{H}_{\mathrm{b}}$ as a function of $\xi_{\mathrm{o}}$. . . . . . . . . . . . . . 104

$\mathrm{X}_{\mathrm{t}} / \mathrm{H}_{\mathrm{b}}$ as a function of $\xi_{\mathrm{b}}$. . . . . . . . . . . . . 105

$\mathrm{X}_{\mathrm{t}} / \mathrm{H}_{\mathrm{o}}$ as a function of $\xi_{0}$. . . . . . . . . . . . . . . 106

Exponential relation for $\mathrm{A}_{\mathrm{v}} / \mathrm{H}_{\mathrm{o}}^{2}$ as a function of $\xi_{0}$. . . . . 107

Linear relation for $\mathrm{A}_{\mathrm{v}} / \mathrm{H}_{\mathrm{o}}^{2}$ as a function of $\xi_{0}$. . . . . . . 108

Linear relation for $\mathrm{A}_{\mathrm{v}} / \mathrm{H}_{\mathrm{o}}^{2}$ as a function of $\xi_{\mathrm{b}}$. . . . . . . 108

Wave height as a function of horizontal distance . . . . . . . 110

$\mathrm{R} / \mathrm{H}_{\mathrm{o}}$ as a function of $\beta_{1}$. . . . . . . . . . . . . . 113

$\mathrm{R} / \mathrm{H}_{\mathrm{o}}$ as a function of $\xi_{0}$. . . . . . . . . . . . . 113 
$60 \mathrm{R} / \mathrm{H}_{\circ}$ as a function of $\xi_{0}$ using $\mathrm{m}$ as the predominant angle... . . . . . . . . . . . . . . 114 $\mathrm{R} / \mathrm{H}_{\circ}$ for plane-slope cases as a function of $\xi_{0}$. . . . . . . 114 Wave at incipient breaking for $\mathrm{H}_{0} / \mathrm{L}_{\mathrm{o}}=0.05$, $\beta_{1}=5 \mathrm{deg}, \beta_{3}=20 \mathrm{deg}$

Wave at incipient breaking for $\mathrm{H}_{\mathrm{o}} / \mathrm{L}_{\mathrm{o}}=0.05$, $\beta_{1}=10 \mathrm{deg}, \beta_{3}=20 \mathrm{deg} \cdot . \cdot 0^{\circ} \cdot \dot{0}^{\circ}$

Wave at incipient breaking for $\mathrm{H}_{\mathrm{o}} / \mathrm{L}_{\mathrm{o}}=0.05$, $\beta_{1}=15 \mathrm{deg}, \beta_{3}=20 \mathrm{deg}$

$\mathrm{H}_{\max }, \mathrm{H}_{\mathrm{s}}$, and $\mathrm{H}_{\mathrm{rms}}$ as a function of distance, $\mathrm{m}=1 / 30$

$\mathrm{H}_{\max }, \mathrm{H}_{\mathrm{s}}$, and $\mathrm{H}_{\mathrm{rms}}$ as a function of distance, $\beta_{1}=5 \mathrm{deg}$

$\mathrm{H}_{\max }, \mathrm{H}_{\mathrm{s}}$, and $\mathrm{H}_{\mathrm{rms}}$ as a function of distance, $\beta_{1}=10 \mathrm{deg}$

$\mathrm{H}_{\max }, \mathrm{H}_{\mathrm{s}}$, and $\mathrm{H}_{\mathrm{rms}}$ as a function of distance, $\beta_{1}=15 \mathrm{deg}$

Predicted $\mathrm{H}_{\max } /\left(\mathrm{H}_{\mathrm{s}}\right)_{0}$ of Goda (1975) $(A=0.18)$ as a function of measured $H_{\max } /\left(H_{s}\right)$ 。

Predicted $\left(\mathrm{H} / \mathrm{H}_{\mathrm{s}}\right)_{\circ}$ of Goda $(1975)(\mathrm{A}=0.12)$ as a function of measured $\left(\mathrm{H} / \mathrm{H}_{\mathrm{s}}\right)_{\text {。 }}$

Predicted $\mathrm{H}_{\mathrm{rms}} /\left(\mathrm{H}_{\mathrm{s}}\right)_{\circ}$ of Goda $(1975) \quad(\mathrm{A}=0.09)$ as a function of measured $\left(\mathrm{H}_{\mathrm{rms}} / \mathrm{H}_{\mathrm{s}}\right)$ 。

$\mathrm{H}_{\mathrm{rms}} / \mathrm{h}$ as a function of distance, $\beta_{1}=5 \mathrm{deg}$

$\mathrm{H}_{\mathrm{rms}} / \mathrm{h}$ as a function of distance, $\beta_{1}=10 \mathrm{deg}$

$\mathrm{H}_{\mathrm{rms}} / \mathrm{h}$ as a function of distance, $\beta_{1}=15 \mathrm{deg}$

$\mathrm{R} /\left(\mathrm{H}_{\mathrm{o}}\right)_{\mathrm{s}}$ as a function of $\xi_{0} \cdot \beta_{1} \cdot \dot{0}^{\circ}$

$\mathrm{R} /\left(\mathrm{H}_{\mathrm{o}}\right)_{\mathrm{s}}$ as a function of $\xi_{0}$ using $\mathrm{m}=1 / 30$ as the predominant angle

Range between maximum and minimum values of $\gamma_{b}$ as a function of $\mathrm{H}_{\mathrm{o}} / \mathrm{L}_{\mathrm{o}}$ 
No.

Page

B20

Case 10410 at incipient breaking

B11 


\section{CONVERSION FACTORS, NON-SI TO SI (METRIC)}

UNITS OF. MEASUREMENTS

Non-SI units of measurement used in this report can be converted to SI (metric) units as follows:

Multiply

degrees (angle)

feet

inches

square feet
By

0.01745329

0.3048

2.54

0.0929
To Obtain

radians

metres

centimetres

square metres 


\section{LABORATORY STUDY ON MACRO-FEATURES OF WAVE \\ BREAKING OVER BARS AND ARTIFICIAL REEFS}

\section{PART I: INTRODUCTION}

\section{Background}

1. Wave breaking is the most dynamic phenomenon in the nearshore zone. A wave approaching a beach enters shallow water, which causes it to become steeper and shorter. The wave eventually becomes unstable, and the crest either spills down or plunges over its front face, releasing its kinetic and potential energy. The crest of a spilling breaker rolls down the front face of the wave and creates intense turbulence at the surface. A plunging breaker crashes into the water surface shoreward of it, displacing a large volume of water that also plunges shoreward. The vortex of entrained air created by the overturning crest penetrates below the water surface. Plunging and, to a lesser extent, spilling waves create turbulence and currents that mobilize and transport beach sediment. Longshore currents, driven by waves breaking at an oblique angle to shore, transport sediment along the coast. Cross-shore currents generated by breaking waves move sediment across shore to form bars. The point of wave breaking and plunging varies according to the height and length of the incident waves, as well as the beach slope and water level (tidal stage). Thus, bars are constantly changing shape and location, moving seaward in storms and shoreward under calmer conditions. Coastal structures such as breakwaters and jetties are constantly subjected to breaking wave forces. Forces exerted by breaking waves can crack concrete dolosse and displace armor units weighing several tons.

2. Placement of clean dredged material offshore in linear berm configurations to both protect and nourish the beach has become an area of recent interest (McLellan 1990). Depending on depth and orientation, the presence of offshore berms may alter the incident wave characteristics and influence the breaking wave properties. Although offshore berms have desirable qualities in concept, research in this field has been fairly limited.

3. Breaking waves also provide a recreational environment for surfers. Recently, a surfing reef (shoal) was designed to be placed at Patagonia, California, and the project is in the final stages (Pratte 1989). Walker (1974a) 
stated the bottom configurations parameters that most influenced favorable surfing waves were the seaward slope, width, and orientation of the shoal to the incident waves.

4. It is clear that knowledge of breaking wave properties is essential in design and placement of coastal structures, as well as for prediction of shoreline movement and beach profile change. Breaking waves have been examined in numerous laboratory studies, but all major experiment programs were conducted on plane slopes. Relationships for breaking wave properties have been developed from such studies, but the presence of a bar or a subsurface structure, such as an offshore berm or an artificial reef, is expected to change the character of breaking. This study departs from previous investigations in that it concentrates on wave breaking over barred profiles with a fixed bottom.

5. Breaking wave characteristics may be studied on a microscale, involving basic fluid dynamic properties such as turbulence, bottom shear stresses, and individual water particle velocities and accelerations; or on a macroscale, involving standard engineering parameters such as wave height and period, water depth, and plunge distance. This study concentrates on macrofeatures of wave breaking over barred profiles in order to reveal functional dependencies and properties of direct coastal engineering interest.

\section{Purpose}

6. The purpose of this study is to examine the macro-features of wave breaking over bars and artificial reefs. Principal wave properties considered are:

a. Breaker type.

b. Wave height and water depth at breaking.

c. Plunge and splash distance.

d. Vortex area.

e. Wave decay.

f. Reflection.

g. Wave runup.

Because this study differs significantly from previous studies of breaking waves by examining the effects of bars on wave breaking, empirical relationships developed from measurements will have immediate input to other 
activities, such as numerical models for simulating beach profile change and studies of breaker wave height decay. Measurements of breaking wave properties over bars and offshore berms and reefs on a microscale are also essential, but are left to future work.

7. The experiment was conducted in a wave tank containing a 1 on 30 concrete slope. Bars and reefs of fixed geometry and constructed of wood were placed on the slope to investigate wave breaking under natural conditions with bars and engineered conditions with artificial reefs. Wave breaking was examined over a range of bar and reef geometries, wave periods, and wave heights for a fixed water level. In nature, breaking waves change the geometry and size of the bar, and these changes feed back to alter the breaking wave characteristics. Bars subjected to steady, monochromatic waves and fixed water levels develop an equilibrium profile (Kraus and Larson 1988; Larson, Kraus, and Sunamura 1989; Larson and Kraus 1989). However, in reality bars are subjected to irregular waves and fluctuating water levels, so an equilibrium profile is only approached and never reached. Consequently, bars and waves are continuously interacting with each other. The present study focuses on the control that bars and artificial reefs exert on breaking waves.

8. This study pertained only to macro-features of wave breaking; microfeatures of bottom velocity, turbulence, and bottom shear stresses were not addressed. Also, the macro-features of wave celerity, return flow velocity, and the important influence of wind on wave breaking, as discussed by Douglass and Weggel (1989), were not obtained in this study. Although return flow was not measured, qualitative observations and implications of the return flow are discussed in Part IV. Data are available from the experiments to examine wave celerity, but because of the considerable data analysis involved, it is left for future work.

\section{Content}

9. Part II contains a review on essential properties of breaking waves, including breaker type, breaker indices, reflection, plunge distance, wave decay, and runup. Part III describes the design and execution of the experiment, and Part IV presents major results. Conclusions and recommendations for future study are given in Part $V$. Wave data from previous studies used in the present study and data generated in the present study are 1 isted in 
Appendix A. Sketches of wave forms from this study are presented in Appendix B, and wave decay data are presented in Appendix C. Notation is listed in Appendix D. 


\section{PART II: REVIEW OF RELATED STUDIES}

10. This chapter reviews previous studies on breaking waves to identify principal variables and trends for comparison with results of the present study. Main topics reviewed are breaker type, breaker index, plunge distance, breaker vortex, wave decay, and runup. Previous studies concentrated on plane sloping beaches; therefore, a background of these topics is necessary to determine the effect, if any, of bars and artificial reefs on the various wave breaking properties. The literature on breaking wave properties is vast, and only the more pertinent studies are included. Original analysis of the previous data is made at the end of the chapter.

\section{Breaker Type}

11. "Breaker type" refers to the form of a depth-limited wave at breaking and has an influence on other breaking wave properties. Although there are several classifications of breaker type, it is generally accepted that waves break by spilling (Figure 1), plunging (Figure 2), collapsing (Figure 3 ), or surging. These photographs were taken during the present study, and the case number is explained in Part IV. Galvin (1968) defined the following terminology; spilling breakers occur if the wave crest becomes unstable and flows down the front face of the wave producing a foamy water surface; plunging breakers occur if the crest curls over the front face and falls into the base of the wave, resulting in a high splash; collapsing breakers occur if the crest remains unbroken while the lower part of the front face steepens and then falls, producing an irregular turbulent water surface; surging breakers occur if the crest remains unbroken and the front face of the wave advances up the beach with minor breaking. Breaker type is controlled by the bottom slope and deepwater wave steepness. The deepwater steepness is defined as the ratio of deepwater wave height $\mathrm{H}_{0}$ and deepwater wavelength $\mathrm{L}_{\mathrm{o}}$, which can be calculated by linear wave theory as:

$$
L_{0}=\frac{g T^{2}}{2 \pi}
$$




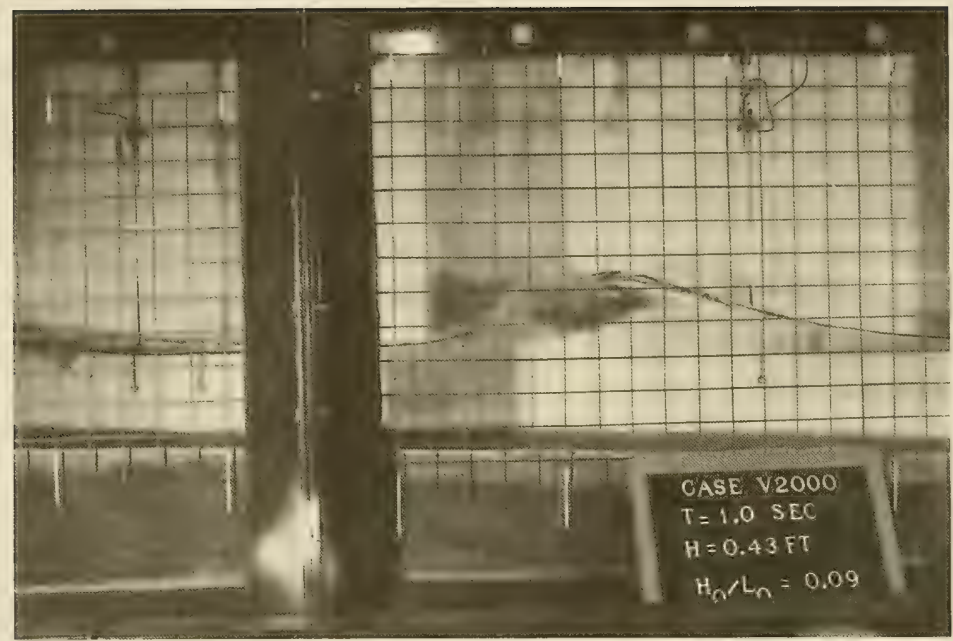

Figure 1. Spilling wave

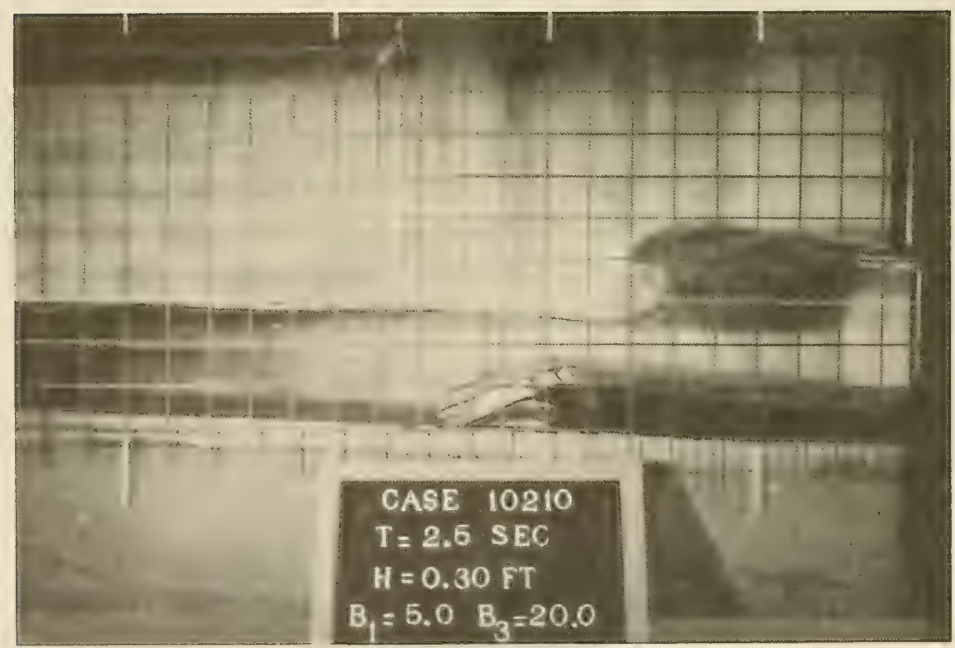

Figure 2. Plunging wave 


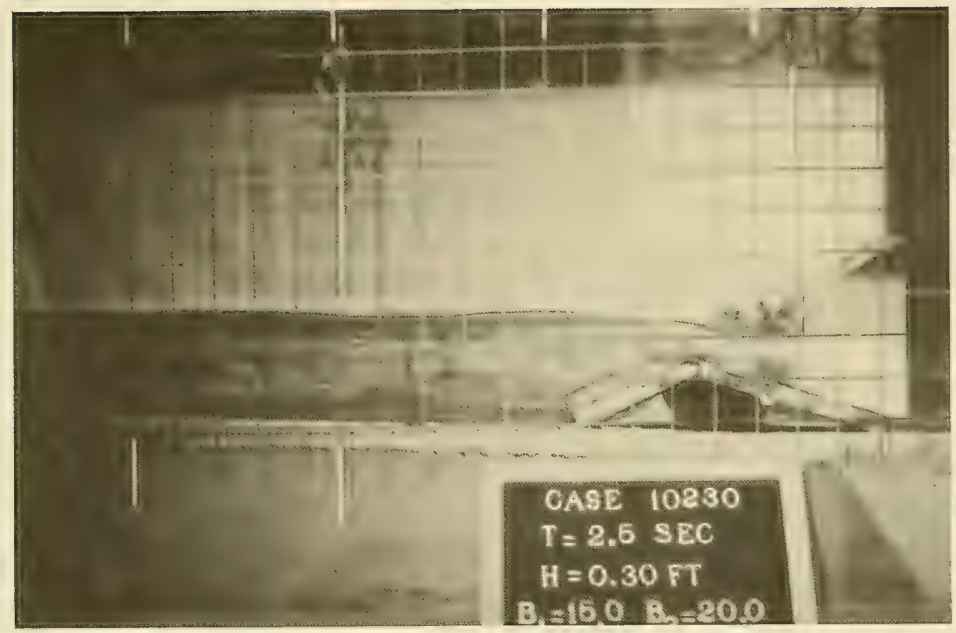

Figure 3. Collapsing wave

where $g$ is acceleration due to gravity, and $T$ is the wave period.

\section{$\underline{\text { Review }}$}

12. Patrick and Wiegel (1954) classified breaker type as spilling, plunging, and surging. Patrick and Wiegel observed in the field that breaker type depended primarily upon beach slope $m$ and deepwater wave steepness. They determined spilling breakers occur for large values of $\mathrm{H}_{\mathrm{o}} / \mathrm{L}_{\mathrm{o}}$ on flat slopes, surging breakers occur for small values of $\mathrm{H}_{\mathrm{o}} / \mathrm{L}_{\mathrm{o}}$ on very steep slopes, and plunging breakers occur between the two extremes.

13. Galvin (1968) introduced collapsing breaker type as an intermediate form of breaker between plunging and surging waves. Galvin defined two parameters, the "offshore parameter" and the "inshore parameter," to classify breaker type, expressed in terms of the beach slope and wave steepness. Transition values were found to be

$$
\begin{array}{rr}
\text { surging-collapsing if } & \frac{\mathrm{H}_{\mathrm{o}}}{\mathrm{L}_{\mathrm{o}} \mathrm{m}^{2}}<0.09 \\
\text { plunging if } 0.09<\frac{\mathrm{H}_{\mathrm{o}}}{\mathrm{L}_{\mathrm{o}} \mathrm{m}^{2}}<4.80
\end{array}
$$


for the offshore parameter, and

$$
\begin{array}{ll}
\text { surging-collapsing if } & \frac{\mathrm{H}_{\mathrm{b}}}{\mathrm{mL}_{\mathrm{o}}}<0.019 \\
\text { plunging if } 0.019<\frac{\mathrm{H}_{\mathrm{b}}}{\mathrm{mL}_{\mathrm{o}}}<0.427 \\
\text { spilling if } & \frac{\mathrm{H}_{\mathrm{b}}}{\mathrm{mL}_{\mathrm{o}}}>0.427
\end{array}
$$

for the inshore parameter, in which $H_{b}$ is the breaking wave height.

14. Battjes (1975) used the Iribarren number (Iribarren and Nogales 1947), commonly called the surf similarity parameter, to describe breaker type. The surf similarity parameter $\xi$ is defined as

$$
\xi=\frac{\mathrm{m}}{\left(\frac{\mathrm{H}}{\mathrm{L}_{\mathrm{o}}}\right)^{1 / 2}}
$$

where $H$ is wave height. The offshore parameter of Galvin (1968) can be written as $\xi_{0}^{-2}$ by specifying $H_{0}$ as the wave height. Battjes converted the transition values of Galvin to values of $\xi_{0}$, resulting in

$$
\begin{array}{rr}
\text { surging or collapsing if } & \xi_{0}>3.3 \\
\text { plunging if } & 0.5<\xi_{0}<3.3 \\
\text { spilling if } & \xi_{0}<0.5
\end{array}
$$

Battjes defined an inshore parameter $\xi_{b}=m\left(H_{b} / L_{0}\right)^{-1 / 2}$ and, by reanalyzing the Galvin data, the following transition values were determined:

$$
\begin{array}{rr}
\text { surging or collapsing if } & \xi_{\mathrm{b}}>2.0 \\
\text { plunging if } 0.4<\xi_{\mathrm{b}} & <2.0 \\
\text { spilling if } & \xi_{\mathrm{b}}<0.4
\end{array}
$$


15. Breaker type is a function of the bottom slope and deepwater wave steepness. Spilling waves occur if the beach slope is flat and wave steepness is large. Plunging breakers are expected for either small wave steepness on mild slopes or large wave steepness on steep slopes. Collapsing and surging breakers occur if wave heights are low in relation to wavelength and slopes are steep.

16. Dimensionless parameters based on beach slope and deepwater wave steepness were developed by Galvin (1968) and Battjes (1975) to quantify breaker type. These parameters are also used to predict other wave breaking properties, such as breaker indices, plunge distance, wave reflection, and runup, as will be discussed.

\section{Wave Reflection}

17. A structure may dissipate or reflect wave energy, or both. The structure may be natural, such as beaches, bars, and reefs, or engineered, such as breakwaters, jetties, and seawalls. Wave reflection is quantified by the reflection coefficient $K_{r}$, which is defined as

$$
\mathrm{K}_{\mathrm{r}}=\frac{\mathrm{H}_{\mathrm{r}}}{\mathrm{H}_{\mathrm{i}}}
$$

in which $\mathrm{H}_{\mathrm{r}}$ and $\mathrm{H}_{\mathrm{i}}$ are the heights of the reflected wave and incident wave, respectively, at a specified point.

\section{Review}

18. Miche (1951) developed a theoretical relation for $K_{r}$ for smooth plane slopes. He assumed that the portion of wave energy that was reflected corresponded to a critical deepwater wave steepness and that wave energy that exceeded this critical value was dissipated. The wave reflection coefficient was expressed as:

$$
K_{r}=\left\{\begin{array}{cl}
\left(\frac{H_{o}}{L_{0}}\right)_{c r}\left(\frac{H_{0}}{L_{0}}\right)^{-1} & \frac{H_{o}}{L_{o}}>\left(\frac{H_{o}}{L_{o}}\right)_{c r} \\
1 & \frac{H_{o}}{L_{o}}<\left(\frac{H_{o}}{L_{o}}\right)_{c r}
\end{array}\right.
$$


where $\left(H_{o} / L_{o}\right)_{c r}$ is the critical wave steepness to obtain complete reflection and is defined as:

$$
\left(\frac{\mathrm{H}_{\mathrm{o}}}{\mathrm{L}_{\mathrm{o}}}\right)_{\mathrm{cr}}=\left(\frac{2 \beta}{\pi}\right)^{1 / 2} \frac{\sin ^{2} \beta}{\pi}
$$

in which $\beta$ is the slope angle in degrees.

19. Battjes (1975) reexpressed Miche's (1951) equation to obtain $K_{r}$ as a function of the surf similarity parameter. The resulting equation was:

$$
\mathrm{K}_{\mathrm{r}}=0.1 \xi_{\mathrm{o}}^{2}
$$

Battjes compared Equation 10 with data collected by Moraes (1971) and found good agreement with the data for breaking waves and $\xi_{0}<2.5$.

20. Ahrens (1987) conducted experiments to determine the stability, transmission, reflection, and energy dissipation characteristics of reef breakwaters. Ahrens defined the reef reflection parameter $P$ as

$$
\mathrm{P}=\frac{\mathrm{z}^{2} \mathrm{~L}_{\mathrm{s}}}{\mathrm{A}_{\mathrm{b}} \mathrm{h}_{\mathrm{s}}}
$$

where

$$
z=\text { height of reef }
$$

$L_{\mathrm{s}}$ and $\mathrm{h}_{\mathrm{s}}=$ wavelength and depth at toe of reef, respectively

$A_{b}=$ cross-sectional area of reef

Ahrens performed a regression analysis to relate wave reflection to the reef reflection parameter and obtained

$$
\mathrm{K}_{\mathrm{r}}=\frac{1}{\left(1.0+8.284 \mathrm{P}^{-0.951}\right)}
$$

The coefficient of determination for Equation 12 was 0.8 .

\section{Summary}

21. Equation 8 (Miche 1951) and Equation 10 (Battjes 1975) include beach slope and deepwater wave steepness to estimate the reflection coefficient. Equation 12 (Ahrens 1987) was developed for reef breakwaters, which differ from the bars and reefs modeled in the present study in that reef 
breakwaters are larger and permeable, have flatter crests, and are not always submerged. However, the reef reflection parameter relates area and height of the structure to reflection and was considered appropriate to examine for describing reflection in the present study.

\section{Breaker Index}

22. A schematic of a wave at incipient breaking is illustrated in Figure 4. Breaking is shown to take place over an idealized bar, where $h_{b}$ is
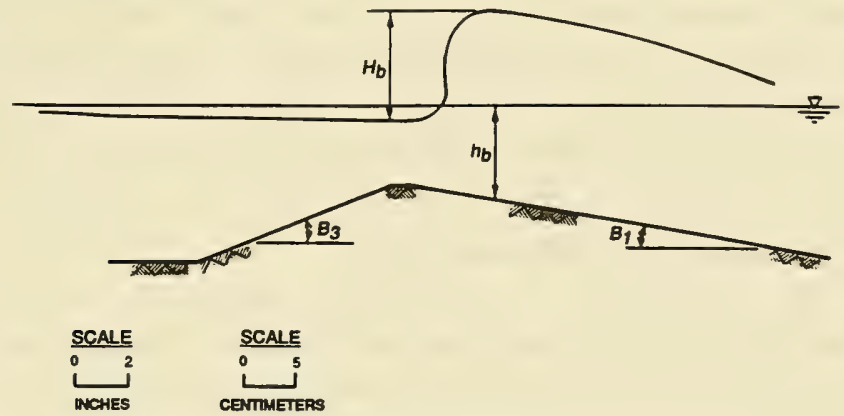

Figure 4. Definition sketch of wave at incipient breaking

the water depth at breaking, $\beta_{1}$ is the seaward bar angle, and $\beta_{3}$ is the shoreward bar angle. The break point can be defined in several ways. Singamsetti and Wind (1980) listed possible definitions as:

a. The point where the wave cannot further adapt to the changing bottom configuration and starts to disintegrate.

b. The point where the horizontal component of the water particle velocity at the crest becomes greater than the wave celerity.

c. The point where the wave height is maximum.

d. The point where part of the wave front becomes vertical.

e. The point where the radiation stresses start to decrease.

f. The point where the water particle acceleration at the crest tends to separate the particles from the water surface.

g. The point where the pressure at the free surface given by the Bernoulli equation is incompatible with the atmospheric pressure. 
In the present study, after considerable inspection of videotapes of breaking waves, the break point was defined as the point where any portion of the forward face of the wave became vertical. Regardless of which method is used, defining the break-point location is rather subjective because a wave does not always transform from a shoaling wave to a broken wave in an abrupt manner. Judgment is always involved; therefore, it is important to state how the break point was determined in reporting of results. Unfortunately, not all authors specify this.

23. It is also important to state the datum at which the depth at breaking was measured. Note from Figure 4 that the water depth at breaking $h_{b}$ is measured from the still-water level (SWL) under the crest of the wave. This definition was used in all tests in the present study, although $h_{b}$ has also been measured from the mean water level (MWL) in some studies. The equipment available for data analysis in the present study made the SWL more convenient to use as a datum.

\section{Review of monochromatic wave studies}

24. Many studies have been performed to develop relationships to denote the wave height at breaking. The term "breaker index" is used to describe nondimensional breaker height. The two common indices are of the form

$$
\gamma_{b}=\frac{H_{b}}{h_{b}}
$$

in which $\gamma_{\mathrm{b}}$ will be called the breaker depth index, and

$$
\Omega_{\mathrm{b}}=\frac{\mathrm{H}_{\mathrm{b}}}{\mathrm{H}_{\mathrm{o}}}
$$

in which $\Omega_{\mathrm{b}}$ will be called the breaker height index.

25. Although the present study involves only periodic waves, most earlier studies on breaker indices were conducted with solitary waves. Two widely quoted studies are noted here. McCowan (1891) theoretically determined the value of $\gamma_{b}$ for a solitary wave on a horizontal bottom as 0.78. Munk (1949) derived the following expression for breaker height index from solitary wave theory: 


$$
\Omega_{\mathrm{b}}=0.3\left(\frac{\mathrm{H}_{\mathrm{o}}}{\mathrm{L}_{\mathrm{o}}}\right)^{-1 / 3}
$$

The relationships of McCowan (1891) and Munk (1949) have been widely accepted in the past because of the hypothesis that periodic waves near the break point behave as solitary waves. The following paragraphs present selected relationships developed for breaker indices using periodic waves.

26. The first major laboratory experiment on breaking waves was conducted by Iversen (1952). Iversen generated periodic waves of steepness in the range of $0.0025<\mathrm{H}_{\mathrm{o}} / \mathrm{L}_{\mathrm{o}}<0.0901$ on four different uniform beach slopes $(1 / 10,1 / 20,1 / 30$, and $1 / 50)$ and developed curves for the breaker height index versus deepwater wave steepness. The curves show $\Omega_{\mathrm{b}}$ decreasing with increasing $\mathrm{H}_{\mathrm{o}} / \mathrm{L}_{\mathrm{o}}$. Iversen also noted breaker height was higher for the steeper slopes. This experiment provided breaker data over a broad range of slopes and wave steepnesses, and the data set is often referenced in breaking wave studies.

27. Ippen and Kulin (1955) conducted experiments with solitary and oscillatory waves and concluded that oscillatory waves do not behave as solitary waves near the break point because of backwash from preceding waves. They also noted $\gamma_{b}$ increased as wave period increased, which implies the breaker depth index increases as $H_{0} / L_{o}$ decreases, since $L_{o}$ is directly related to wave period.

28. Galvin (1969) performed laboratory experiments with periodic waves on three different uniform slopes $(1 / 5,1 / 10,1 / 20)$ and found that if his data were combined with the Iversen (1952) data,

$$
\begin{array}{cc}
\frac{1}{\gamma_{b}}=0.92 & m>0.07 \\
\frac{1}{\gamma_{b}}=1.40-6.85 m & m \leq 0.07
\end{array}
$$

29. Collins and Weir (1969) derived the following expression from linear theory and experimental data of Suquet (1950), Iversen (1952), and Hamada (1963): 


$$
\gamma_{\mathrm{b}}=0.72+5.6 \mathrm{~m}
$$

Equation 18 described the experimental data well for slopes $1 / 20$ and milder. 30. Goda (1970) developed curves from existing laboratory data for beach slopes ranging from $1 / 10$ to $1 / 50$. Goda noted that bottom slope affects not only breaker height, but also breaker depth.

31. Weggel (1972) developed a relationship for $\Omega_{\mathrm{b}}$ based on the form of Munk's (1949) relationship for $\Omega_{b}$ as

$$
\Omega_{\mathrm{b}}=F(m)\left(\frac{\mathrm{H}_{\mathrm{o}}}{\mathrm{L}_{\mathrm{o}}}\right)^{-1 / 3}+\mathrm{G}(\mathrm{m})
$$

Weggel determined the functions $F(m)$ and $G(m)$ empirically from the Iversen (1952) data, where

$$
\begin{gathered}
F(m)=D_{1}[1+m-G(m)] \\
G(m)=\left[D_{1}(1+m)-D_{2}\left(1.715-0.185 e^{-28 m}\right)\right]
\end{gathered}
$$

and

$$
\begin{gathered}
D_{1}=(0.01+0.5 m)^{1 / 3} \\
D_{2}=\left(0.01-0.01 e^{-28 m}\right)^{1 / 3}
\end{gathered}
$$

where $D_{1}$ and $D_{2}$ are empirical functions for breaker height (Weggel 1972). Wegge1 commented that use of Equation 19 became questionable for $\mathrm{m}>1 / 10$ and $\mathrm{H}_{\mathrm{o}} / \mathrm{L}_{\mathrm{o}}>0.06$.

32. Weggel (1972) also developed an equation for the breaker depth index from previous laboratory data (Iversen 1952, Reid and Bretschneider 1953, Galvin 1969, Weggel and Maxwell 1970, and Jen and Lin 1971) collected on slopes of $1 / 5,1 / 10,1 / 15,1 / 20$, and $1 / 50$. The resulting relationship is expressed as

$$
\gamma_{b}=b(m)-a(m) \frac{H_{b}}{L_{o}}
$$


for

$$
\begin{aligned}
& a(m)=6.97\left(1-e^{-19 m}\right) \\
& b(m)=\frac{1.56}{\left(1+e^{-19.5 m}\right)}
\end{aligned}
$$

where $a(m)$ and $b(m)$ are empirical coefficients. Equations 24 and 25 are presented in nondimensional form, but the original equation of Weggel (1972) was given in US customary units.

33. The study by Jen and Lin (1971) was conducted to determine impact pressures on a cylindrical tube placed in the tank. Breaking wave properties obtained from these tests are expected to differ from results from other tests used in the Weggel (1972) analysis. Data obtained from Reid and Bretschneider (1953) were used as transition values from limiting wave steepness to depthlimited breaking. A portion of the data reported by Reid and Bretschneider were from Danel (1952), a study on limiting clapotis.

34. Weggel (1972) determined $a(m)$ and $b(m)$ by assigning minimum and maximum limits to $\gamma_{\mathrm{b}}$. The theoretical value of a solitary wave, $\gamma_{\mathrm{b}}=0.78$, from McCowan (1891), was used as the minimum breaker depth index, occurring for $m=0$. As $m$ approached infinity, such as at a vertical wall, $\gamma_{b}$ was assumed to be double the minimum value, 1.56, by adding the incident and perfectly reflected wave heights. Breaking wave height appears on both sides of Equation 24, so an iterative approach must be used to determine $\gamma_{\mathrm{b}}$. Equation 24 can easily be solved with the use of a computer, but otherwise the form is inconvenient for hand calculations.

35. Komar and Gaughan (1973) derived a semiempirical relationship for $\Omega_{\mathrm{b}}$ from linear wave theory where

$$
\Omega_{\mathrm{b}}=0.56\left(\frac{\mathrm{H}_{\mathrm{o}}}{\mathrm{L}_{\mathrm{o}}}\right)^{-1 / 5}
$$

The coefficient 0.56 was determined empirically from laboratory data (Iversen 1952, Galvin 1969, and Komar and Simmons (as reported by Komar and Gaughan 1973)) and from field data (Munk 1949). 
36. Singamsetti and Wind (1980) collected laboratory data and combined the results with data from Galvin (1969) and Iversen (1952). Singamsetti and Wind found

$$
\Omega_{\mathrm{b}}=0.575 \mathrm{~m}^{0.031}\left(\frac{\mathrm{H}_{\mathrm{o}}}{\mathrm{L}_{\mathrm{o}}}\right)^{-0.254}
$$

valid for $0.02<\mathrm{H}_{\mathrm{o}} / \mathrm{L}_{\mathrm{o}}<0.065$ and $1 / 40<\mathrm{m}<1 / 5$, and

$$
\gamma_{b}=0.568 \mathrm{~m}^{0.107}\left(\frac{H_{o}}{L_{o}}\right)^{-0.237}
$$

valid for $0.02<\mathrm{H}_{\circ} / \mathrm{L}_{\mathrm{o}}<0.06$ and $1 / 40<\mathrm{m}<1 / 10$.

37. Sunamura (1981) conducted a laboratory experiment and combined the results with data from Iversen (1952); Bowen, Inman, and Simmons (1969); and Goda (1970) to obtain the simple equation

$$
\gamma_{\mathrm{b}}=1.1 \xi_{\mathrm{o}}^{1 / 6}
$$

which can be written as

$$
\gamma_{b}=1.1 \mathrm{~m}^{1 / 6}\left(\frac{\mathrm{H}_{\mathrm{o}}}{\mathrm{L}_{\mathrm{o}}}\right)^{-1 / 12}
$$

38. Sunamura (1982) obtained the following empirical relation for $\Omega_{b}$ using data collected from small-scale laboratory experiments (Sunamura and Horikawa 1975):

$$
\Omega_{\mathrm{b}}=1.0 \mathrm{~m}^{0.2}\left(\frac{\mathrm{H}_{\mathrm{o}}}{\mathrm{L}_{\mathrm{o}}}\right)^{-0.25}
$$

Sunamura found good agreement between Equation 32 and prototype-scale laboratory experiments (Kajima et al. 1983). 
Summary of breaker depth

39. The variables involved in determining breaker indices are local bottom slope and deepwater wave steepness. Table 1 lists coefficients ( $C_{1}$ and $\mathrm{C}_{2}$ ) and exponents $\left(\mathrm{n}_{1}\right.$ and $\left.\mathrm{n}_{2}\right)$ of the different breaker depth equations described previously for comparison, where the basic equation is of the form:

$$
\gamma_{b}=C_{1} m n_{1}\left(\frac{H_{o}}{L_{o}}\right)^{n_{2}}+C_{2}
$$

Equation 58, based on data collected from previous studies, was developed in the present study and is described at the end of this chapter.

Table 1

Summary of Coefficients and Exponents for

Breaker Depth Index

\begin{tabular}{|c|c|c|c|c|c|}
\hline Source & $\mathrm{C}_{1}$ & $\mathrm{n}_{1}$ & $\mathrm{C}_{2}$ & $\mathrm{n}_{2}$ & $\begin{array}{c}\text { Equation } \\
\text { Number }\end{array}$ \\
\hline McCowan (1891) & 0 & 0 & 0.78 & 0 & -- \\
\hline Galvin (1969) & $\begin{array}{l}0 \\
0\end{array}$ & $\begin{array}{l}0 \\
0\end{array}$ & $\begin{array}{l}1.09 \\
f(m) *\end{array}$ & $\begin{array}{l}0 \\
0\end{array}$ & $\begin{array}{l}16 \\
17\end{array}$ \\
\hline $\begin{array}{l}\text { Collins and } \\
\text { Weir (1969) }\end{array}$ & 5.6 & 1 & 0.72 & 0 & 18 \\
\hline Wegge1 (1972)** & $-a(m)$ & 0 & $b(m)$ & 1 & 24 \\
\hline $\begin{array}{l}\text { Singamsetti and } \\
\text { Wind (1980) }\end{array}$ & 0.568 & 0.107 & 0 & -0.237 & 29 \\
\hline Sunamura (1981) & 1.1 & 0.167 & 0 & -0.083 & 30 \\
\hline Present Study & $-a(m)$ & 0 & $\mathrm{~b}(\mathrm{~m})$ & 1 & 58 \\
\hline
\end{tabular}

* $f(m)=$ function of beach slope.

** $\mathrm{H}_{\mathrm{b}}$ assumed equal to $\mathrm{H}_{\mathrm{o}}$.

40. Figure 5 (a-d) shows a graphical comparison of the different breaker depth equations as a function of deepwater wave steepness for slopes of $1 / 10,1 / 20,1 / 40$, and 1/80. To include Equation 24 (Weggel 1972) in Figure $5(\mathrm{a}-\mathrm{d}), \mathrm{H}_{\mathrm{b}}$ was set equal to $\mathrm{H}_{\mathrm{o}}$. Equation 29 was plotted within the limits specified by Singamsetti and Wind (1980). Equations 16, 17 (Galvin 1969), and 18 (Collins and Weir 1969) are functions of beach slope only and give a constant value of $\gamma_{b}$ regardless of deepwater wave steepness. 


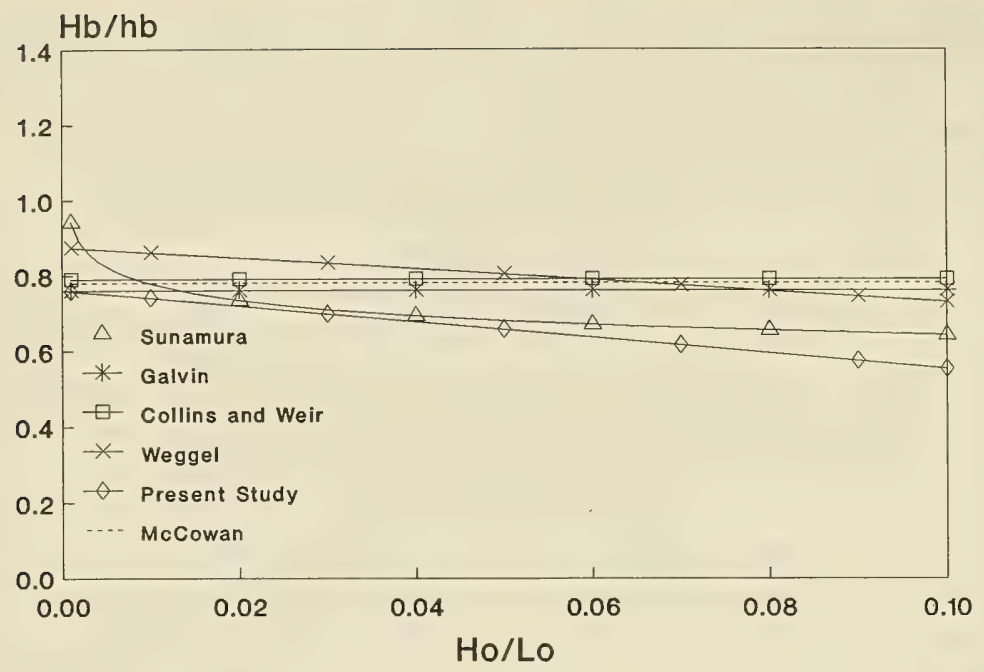

a. $\mathrm{m}=1 / 80$

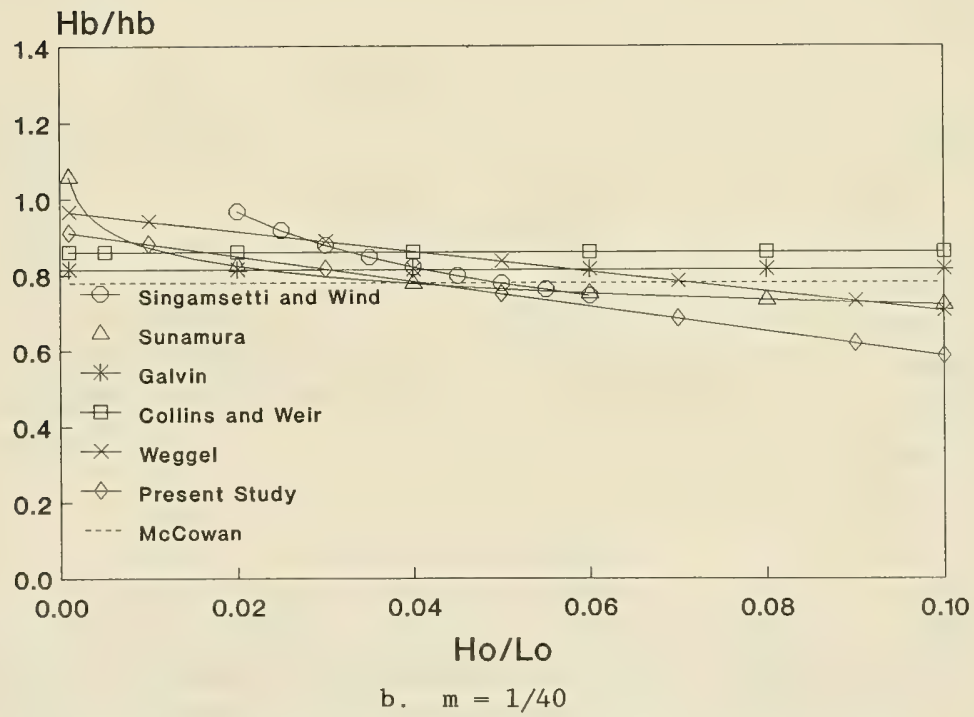

Figure 5. Breaker depth index predictions (Continued) 


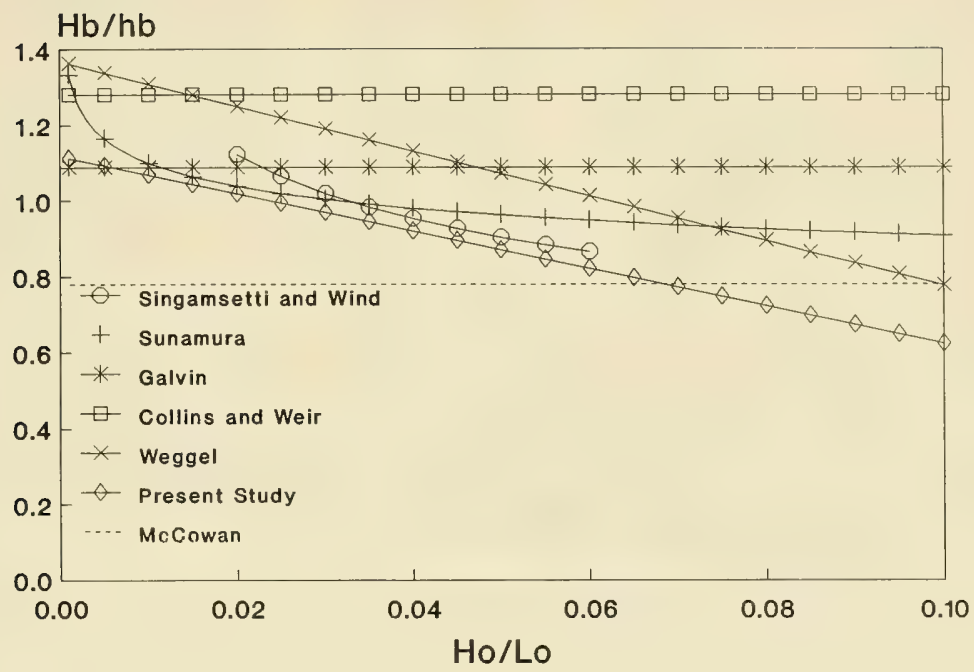

c. $\mathrm{m}=1 / 20$

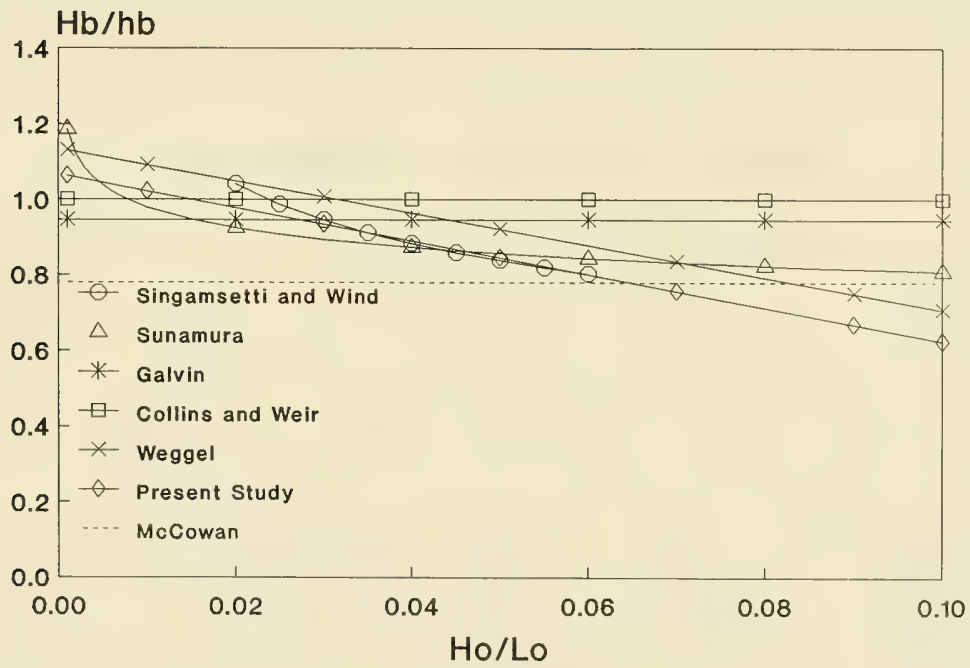

d. $\mathrm{m}=1 / 10$

Figure 5. (Concluded) 
Relationships given by Weggel (1972), Singamsetti and Wind (1980), Sunamura (1981), and the present study show the dependence of $\gamma_{b}$ not only on beach slope, but also on wave steepness. Figure 5 (a-d) illustrates the variability between equations, even though the extensive Iversen (1952) data set was included in the development of all relationships, with the exception of McCowan. The variability between equations may result from different definitions of breaking, experimental variability, and different datums at which depth was measured (MWL or SWL). A11 of the relationships were developed in a logical manner; however, Singamsetti and Wind (1980), Sunamura (1981), and the present study include both beach slope and deepwater wave steepness in the developed equations. Therefore, Equations 29, 30, and 58 are recommended. Use of Equations 29 and 58 should be restricted to values of $m$ and $H_{o} / L_{\circ}$ suggested by Singamsetti and Wind (1980) and the present study, respectively.

\section{Summary of breaker height}

41. Bottom slope and deepwater wave steepness are also used to determine the breaker height index. The basic equation for breaker height can also be expressed in the form of Equation 33. A list of the different coefficients and exponents for breaker height equations are given in Table 2. The equation for $\Omega_{b}$ determined in the present study can be found at the end of this chapter. Figure 6 (a-d) gives a graphical comparison of the different breaker Table 2

\section{Summary of Coefficients and Exponents for}

\section{Breaker Height Index}

\begin{tabular}{|c|c|c|c|c|c|}
\hline Source & $\mathrm{C}_{1}$ & $\mathrm{n}_{1}$ & $\mathrm{C}_{2}$ & $\mathrm{n}_{2}$ & $\begin{array}{l}\text { Equation } \\
\text { Number }\end{array}$ \\
\hline Munk (1949) & 0,30 & 0 & 0 & -0.33 & 15 \\
\hline Weggel (1972) & $F(m)$ & 0 & $G(m)$ & -0.33 & 19 \\
\hline $\begin{array}{l}\text { Komar and } \\
\text { Gaughan (1973) }\end{array}$ & 0.56 & 0 & 0 & -0.20 & 27 \\
\hline $\begin{array}{l}\text { Singamsetti and } \\
\text { Wind }(1980)\end{array}$ & 0.575 & 0.031 & 0 & -0.254 & 28 \\
\hline Sunamura (1982) & 1.0 & 0.2 & 0 & -0.25 & 32 \\
\hline Present Study & $C(m) *$ & 0 & 0 & $\mathrm{n}(\mathrm{m})$ & 61 \\
\hline
\end{tabular}

* $C(m)=$ empirical coefficient in breaker height equation. 


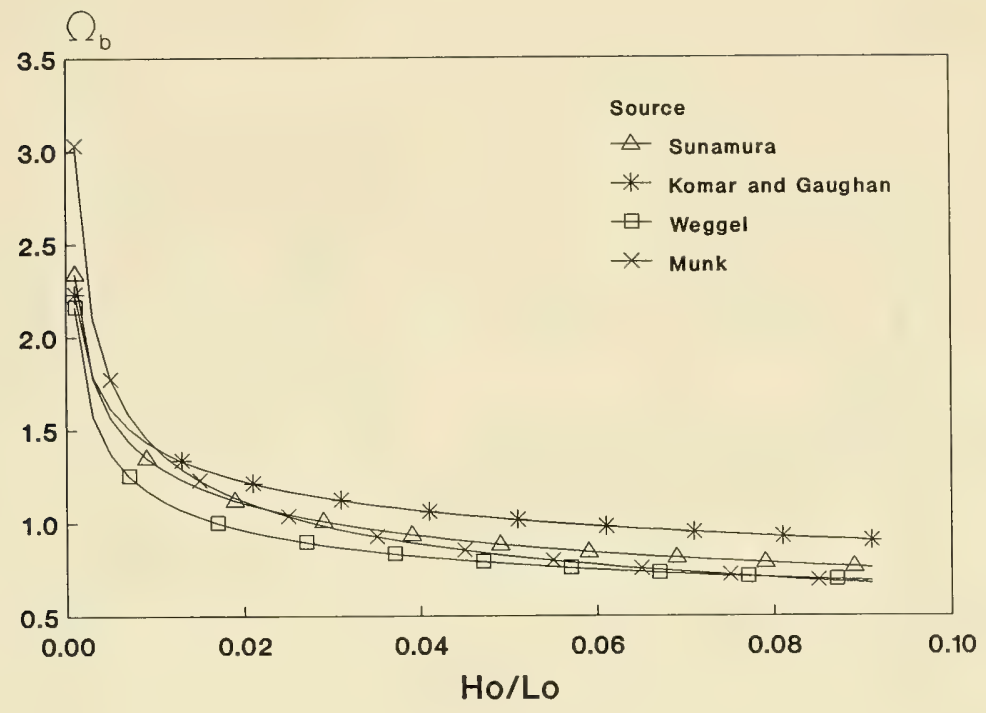

a. $\mathrm{m}=1 / 80$

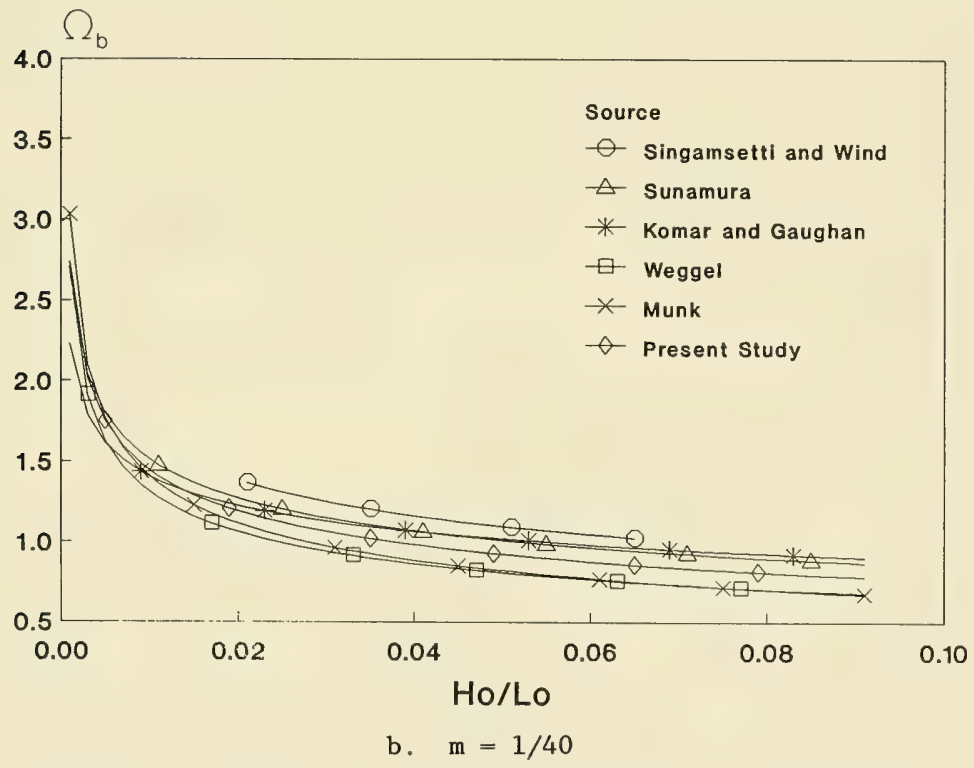

Figure 6. Breaker height index predictions (Continued) 


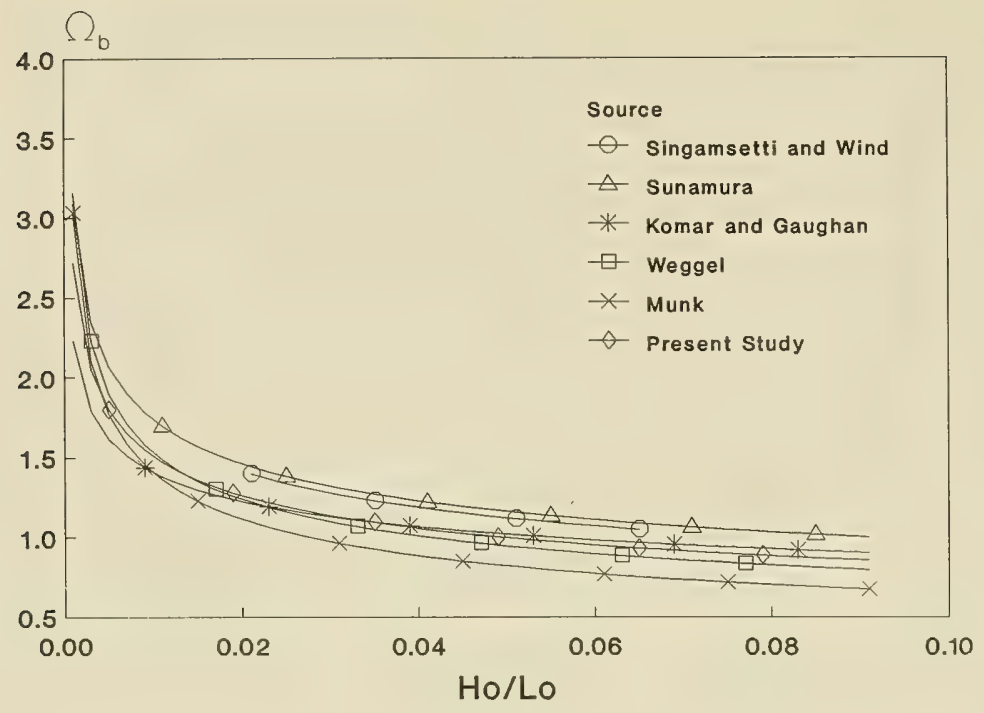

c. $m=1 / 20$

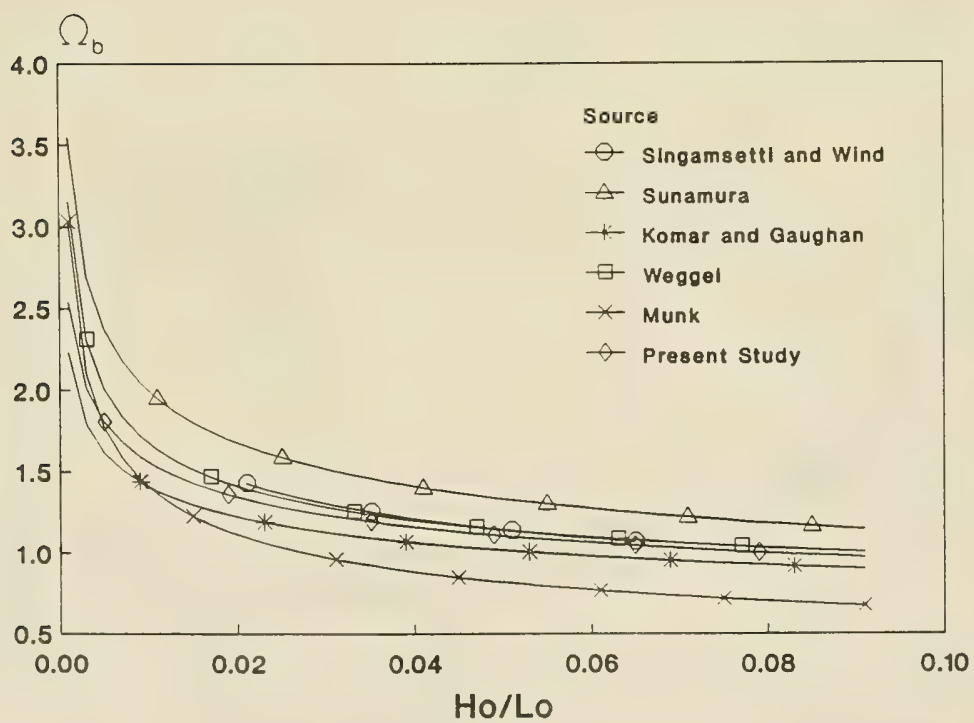

d. $m=1 / 10$

Figure 6. (Concluded) 
height equations as a function of deepwater wave steepness. Breaker height values computed using Equations 15 (Munk 1949) and 27 (Komar and Gaughan 1973) are only a function of $\mathrm{H}_{\mathrm{o}} / \mathrm{L}_{\mathrm{o}}$; therefore, the curves shown in Figure 6 (a-d) for these equations do not change with beach slope. All the curves in Figure 6 (a-d) are fairly consistent in shape and range of values. Since relationships by Wegge1 (1972), Singamsetti and Wind (1980), Sunamura (1982), and the present study include both beach slope and deepwater wave steepness, these equations are recommended. Strictly speaking, use of Equations 19, 28, and 61 should be restricted to the limits given by the respective authors.

Review of irregular wave studies

42. Irregular wave breaking is more complex than monochromatic wave breaking. The incident wave height and wavelength vary from wave to wave, as do the breaking wave height and depth. Most irregular wave breaking models, for example those of Collins (1971), Battjes (1973), and Kuo and Kuo (1974), use a Rayleigh distribution of wave height and truncate the distribution at $\mathrm{H}>\mathrm{H}_{\mathrm{b}}$, in which $\mathrm{H}_{\mathrm{b}}$ is determined from monochromatic breaking wave criteria. Irregular wave breaking and decay are difficult to separate because there is no distinct breaker line, and broken as well as unbroken waves are present through the surf zone.

43. Goda (1975) developed a numerical model for irregular wave deformation based on various laboratory data. Goda used a modified Rayleigh distribution in which the portion of the distribution that represents broken waves $\left(\mathrm{H}>\mathrm{H}_{\mathrm{b}}\right)$ is tapered, rather than cut, which gives a range of breaking wave heights. Breaker height is expressed as:

$$
\frac{\mathrm{H}_{\mathrm{b}}}{\mathrm{H}_{\mathrm{o}}}=\mathrm{A} \frac{\mathrm{L}_{\mathrm{o}}}{\mathrm{H}_{\mathrm{o}}}\left(1-e^{-\mathrm{x}}\right)
$$

where

$$
\mathrm{x}=1.5 \frac{\mathrm{h} \pi}{\mathrm{L}_{\mathrm{o}}}\left(1+\mathrm{K} \tan ^{\mathrm{s}} \mathrm{m}\right)
$$

in which the coefficient $A$ ranges from a maximum of 0.18 to a minimum of 0.12 , the coefficient $K=15$, and $s=4 / 3$. Goda found that $A=0.17$ 
best fit index curves for monochromatic waves previously presented by Goda (1970) and chose the higher A-value to take into account the variability of breaker heights. The lower value was chosen as two-thirds of the higher value. Goda (1975) stated, "The choice was arbitrary, but it has yielded good agreement with laboratory and field data."

44. The relation of Goda (1975) was used by Seelig (1979) to develop curves to estimate nearshore significant wave height over a range of beach slopes and deepwater wave steepnesses. Seelig (1980) used Goda's relationship to present curves to predict the location and magnitude of maximum wave height in the surf zone.

45. Thornton, Wu, and Guza (1985) introduced the term "mean breaker line" for random waves, defined as the location where an averaged wave height is maximum. Waves at the mean breaker line are both broken and unbroken. The averaged wave height used was root-mean-square (rms) wave height $\mathrm{H}_{\mathrm{rms}}$, maximum wave height $H_{\max }$, and the average of the highest one-third of the recorded wave heights $H_{1 / 3}$, also written as $H_{s}$, the significant wave height. Thornton, Wu, and Guza used field data acquired at Torrey Pines and Santa Barbara, California, and laboratory data of Goda (1975); Seelig, Ahrens, and Grosskopf (1983); and Thompson and Vincent (1984) to compare the analytical model of Goda as calculated by Seelig with design curves based on monochromatic waves given in the Shore Protection Manual (SPM) (1977). The model of Goda reasonably estimated $\mathrm{H}_{1 / 3}$ and $\mathrm{H}_{\max }$ for large wave steepness laboratory data, which was expected since the model was based on the same laboratory data. The Goda model overpredicted $\mathrm{H}_{1 / 3}$ for small wave steepnesses, but reasonably predicted $H_{\max }$. The depth at breaking was predicted well for all data. The design curves in the SPM were found to be conservative, especially for small wave steepnesses.

46. Sawaragi, Deguchi, and Park (1989) conducted an experiment with an artificial reef placed in a small wave tank. The reef had a $1 / 30$ slope that extended above SWL and a $1 / 2$ offshore slope. Sawaragi, Deguchi, and Park developed an expression for the A-value of Equation 34 (Goda 1975) for wave height over the reef, but the study concerned energy dissipation rather than breaking wave properties.

Summary

47. Estimation of breaker height and location for irregular waves is difficult, since breaking occurs at different locations with different 
heights. The model of Goda (1975) gives a range of breaking wave heights that has compared well with measurements in the field (Thornton, Wu, and Guza 1985). Although there is no distinct breaker location, the "mean breaker line" gives an indication of where waves break.

\section{Plunge Distance}

48. The crest of a wave at breaking travels some distance shoreward and strikes the water surface (Figure 7), displacing a volume of water which also travels shoreward. Galvin (1969) defined plunge distance as distance from the break point to the crest touchdown point, and splash distance as distance from the crest touchdown point to the splash touchdown point. Sunamura (1987) adds that the plunge point is the "point where breaking waves completely

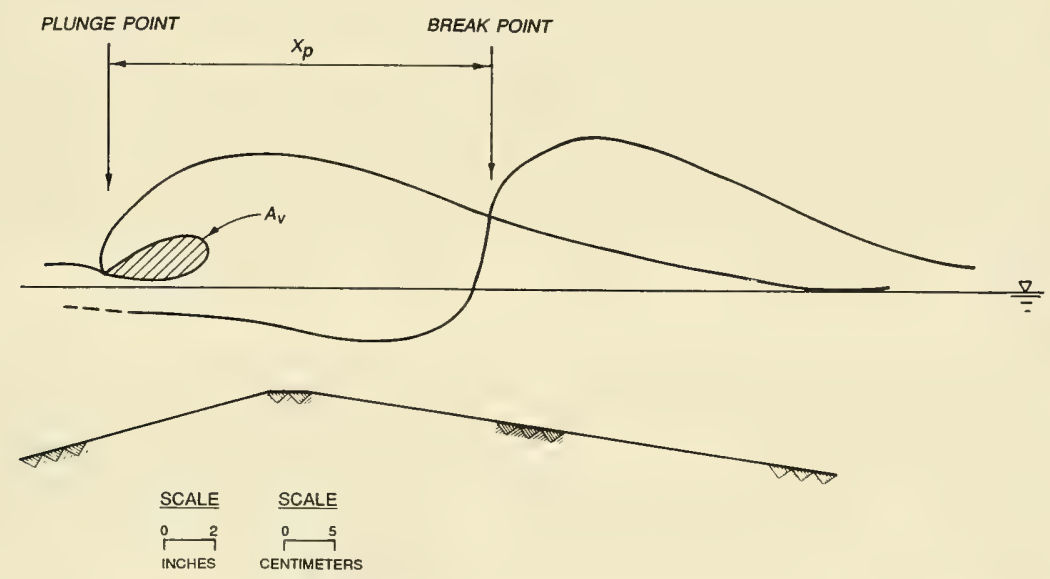

Figure 7. Definition sketch of plunge distance and vortex area

disintegrate as their crest enters the underlying water." Plunge distance is usually associated with plunging waves, but the crests of spilling and collapsing waves also plunge shoreward into the water column and, therefore, have a plunge distance associated with them.

\section{Review}

49. Galvin (1969) conducted laboratory experiments on three planar slopes $(1 / 5,1 / 10,1 / 20)$ and found that plunge distance $X_{p}$ normalized by breaking wave height was given by 


$$
\frac{\mathrm{X}_{\mathrm{p}}}{\mathrm{H}_{\mathrm{b}}}=4.0-9.25 \mathrm{~m}
$$

Galvin noted that $\mathrm{X}_{\mathrm{p}} / \mathrm{H}_{\mathrm{b}}$ values reached as high as 4.5 and the average was 3.0. Splash distance $X_{s}$ was approximately the same as the plunge distance. 50. Weisher and Byrne (1979) filmed breaking waves from a pier at Virginia Beach, Virginia, and measured the plunge distance. Average $\mathrm{x}_{\mathrm{p}} / \mathrm{H}_{\mathrm{b}}$ values were 5.9, and values ranged from 1 to 10 .

51. Singamsetti and Wind (1980) collected plunge distance data in the laboratory and found $\mathrm{x}_{\mathrm{p}} / \mathrm{H}_{\mathrm{b}} \approx 3$ on a $1 / 5$ slope with a range from 4 to 8 on a $1 / 40$ slope. Singamsetti and Wind found that Equation 36 underestimated their data by approximately 50 percent.

52. Visser (1982) conducted laboratory experiments in a basin to measure longshore current. His measurements include plunge distance: Waves were generated at an angle to a $1 / 20$ slope, and Visser found $\mathrm{x}_{\mathrm{p}} / \mathrm{H}_{\mathrm{b}} \approx 6$.

53. Larson and Kraus (1989) tested Equation 36 to predict plunge distance, which was a quantity required as input to a numerical model of beach profile change. Equation 36 was found to underestimate $x_{p}$ for steep bar face slopes. Based on results of Singamsetti and Wind (1980), Larson and Kraus used a constant value of $\mathrm{X}_{\mathrm{p}} / \mathrm{H}_{\mathrm{b}}=3$ in their numerical model. Summary

54. Plunge distance data are limited, and only one relationship has been given to estimate $X_{p}$. However, Equation 36 (Galvin 1969) gives values of $\mathrm{X}_{\mathrm{p}} / \mathrm{H}_{\mathrm{b}}$ of 4 or less, and observations have shown $\mathrm{X}_{\mathrm{p}} / \mathrm{H}_{\mathrm{b}}$ as high as 10 in the field and 8 in the laboratory. These large differences indicate a need for a more reliable relationship to predict plunge distance.

\section{Breaker Vortex}

55. The cavity of entrapped air created by the overturning crest of the wave at the plunge point is called the breaker vortex. The cross-sectional area of the vortex $A_{v}$ is shown in Figure 7. Air and water mix as the vortex penetrates the water column. On a movable bed bottom, sediment is suspended, which either is transported by currents or settles to the bottom as the vortex loses angular momentum, decelerates, and vanishes. 
56. Few studies appear to have been conducted on breaker vortices, although the investigations discussed below indicate that vortices contribute to bar formation, sediment transport, and wave height decay. Two studies are discussed in the following paragraphs. The first study involved wave height decay, and the second study concerned breakpoint bar formation.

Review

57. Sawaragi and Iwata (1974) conducted experiments in a wave tank on a composite slope consisting of a $1 / 18$ foreslope leading to a horizontal section. They examined wave deformation shoreward of the break point and estimated that 15 to 30 percent of the energy of the plunging waves was converted to kinematic energy of the subsurface vortex.

58. Miller (1976) investigated breaker vortices in a series of tests with a tilting wave tank containing a sand-filled bottom. He observed that vortices created by plunging waves might extend from the surface to the bottom, whereas vortices generated by spilling waves were smaller and confined to the region near the surface. The results indicated that bars were formed in the presence of large vortices generated by plunging waves, but bars tended to be eliminated when subjected to spilling waves. Miller commented that the simplified set of wave tank results did not justify immediate extrapolation to the field, but suggested that this was a promising area for further study.

\section{Summary}

59. Sawaragi and Iwata (1974) and Miller (1976) discuss implications of breaker vortices on wave dissipation and sediment movement. Since vortex area cannot be measured easily in the field, the present study attempts to relate vortex area to properties that can be measured easily, such as wave height, period, and local bottom shape.

\section{Wave Height Decay}

60. A broken wave dissipates energy as it progresses shoreward. The wave may remain turbulent to the beach or become stable and reform. A reformed wave will also shoal and eventually break. Wave height decay is needed to calculate radiation stresses, which drive currents, entrain sediment, and create setup in the surf zone. The broken wave height is also needed to design structures to be located in the surf zone. Several models have been developed to predict wave height decay, and some of these are 
described in the following paragraphs to illustrate the various types of models. A more thorough literature review can be found in Smith and Kraus (1988).

\section{$\underline{\text { Review }}$}

61. Wave height decay can be determined from the dissipation of energy in the surf zone. The energy flux equation is written as

$$
\frac{\partial F}{\partial x}=-\varepsilon
$$

in which $F$ is energy flux, $x$ is horizontal distance, and $\varepsilon$ is the energy dissipation rate defined as

$$
\mathrm{F}=\mathrm{EC}_{\mathrm{g}}
$$

where $\mathrm{E}$ is wave energy and $\mathrm{C}_{\mathrm{g}}$ is group speed of the waves defined as

$$
E=\frac{\rho g H^{2}}{8}
$$

in which $\rho$ is water density.

62. Le Méhauté (1963); Divoky, Le Méhauté, and Lin (1970); Battjes and Janssen (1979); Stive (1984); and Svendsen (1984, 1985) assumed the energy dissipation rate of a broken wave was similar to the dissipation rate of a hydraulic jump. The dissipation rate of the hydraulic jump is

$$
\varepsilon=\alpha \frac{\rho g \mathrm{H}^{3}}{4 \mathrm{hT}}
$$

where $\alpha$ is an empirical coefficient and $h$ is the still-water depth. Battjes and Janssen (1979) and Svendsen (1984, 1985) found $\alpha$ equal to unity, indicating the dissipation rate of broken waves and hydraulic jumps is the same. Stive determined $\alpha$ to be a function of beach slope and wave steepness. 
63. Svendsen (1984, 1985) determined $F$ from crude approximations of actual flow in surf zone waves. Svendsen used a nondimensional form of the energy flux $B$ where

$$
\mathrm{B}=\frac{\mathrm{F}}{\rho \mathrm{gCH}^{2}}
$$

and $C$ is wave celerity, equal to $\mathrm{L} / \mathrm{T}$, where $\mathrm{L}$ is wavelength. Svendsen assumed an idealized flow within the wave and the so-called surface roller, and he approximated $B$ by

$$
B=\frac{1}{T} \int_{0}^{T}\left(\frac{\eta}{H^{2}}\right)^{2} d t+\frac{1}{2} \frac{A_{s}}{H^{2}} \frac{h}{L}
$$

in which $\eta$ is the free-surface water elevation, and $A_{s}$ is the crosssectional area of the surface roller. The surface roller is defined as the recirculating part of the flow resting on the front face of the broken wave. Svendsen found a relationship for $A_{s}$ based on experimental data of Duncan (1981) where

$$
A_{s}=0.9 H^{2}
$$

This reduces Equation 42 to

$$
B=\frac{1}{T} \int_{0}^{T}\left(\frac{\eta}{H^{2}}\right)^{2} d t+0.45 \frac{h}{L}
$$

64. Dally (1980) and Dally, Dean, and Dalrymple (1985a, 1985b) derived an equation to calculate the decay of broken waves, in which $\varepsilon$ was assumed to be proportional to the difference between local energy flux $F$ and a stable energy flux $F_{\mathrm{s}}$ : 


$$
\varepsilon=\frac{K}{h}\left(F-F_{s}\right)
$$

in which

$$
F_{s}=\frac{\rho g \Gamma^{2} h^{2} C_{g}}{8}
$$

where $\mathrm{K}$ is a dimensionless decay coefficient, and $\Gamma$ is an empirical stable wave factor equal to the ratio of the stable wave height to water depth.

65. Ebersole (1987) compared the models of Dally (1980); Dally, Dean, and Dalrymple (1985a, 1985b); and Svendsen (1984) to field data collected at Duck, North Carolina. Both models predicted wave height well, especially in the inner surf zone.

66. Mizuguchi (1981) developed a model that allowed for wave deformation on complex beach profiles, as does models of Dally (1980); Svendsen (1984, 1985); and Dally, Dean, and Dalrymple (1985a, 1985b). The approach in modeling the surf zone energy dissipation, which Mizuguchi states is "physically obscure," is to replace molecular viscosity with turbulent eddy viscosity in solving for internal energy dissipation due to viscosity. Model predictions compared well with laboratory data collected on a horizontal beach, a $1 / 10$ plane slope, and a step-type beach.

67. A simpler and more traditional method of estimating wave height in the surf zone is by the expression suggested by Longuet-Higgins and Stewart (1964).

$$
H=\gamma_{b} h
$$

Bowen, Inman, and Simmons (1969) supported the relationship with laboratory data conducted on a 1/12 slope. Equation 47 implies wave height decay is linear; however, laboratory data by Horikawa and Kuo (1967), Street and Camfield (1967), and Van Dorn (1977) indicate decay is steeper than predicted by Equation 47 on gentler bottom slopes.

68. Noting the concave form of the broken wave profile and motivated by analytical studies to use a simple but more accurate prediction of the broken 
wave height than a constant value of $\gamma_{b}$, Smith and Kraus (1988) developed a power law equation for wave height decay as

$$
H=\gamma_{b} h_{b}\left(\frac{h}{h_{b}}\right)^{n}
$$

where $n$ was empirically determined through regression analysis from previously acquired data of Kuo (1965), Horikawa and Kuo (1967), Saeki and Sasaki (1973), Sasaki and Saeki (1974), Van Dorn (1977), Mizuguchi (1981), Maruyama et al. (1983), and Stive (1985). Multiple regression of the data gave:

$$
\mathrm{n}=0.657 \gamma_{\mathrm{b}}+\frac{0.043 \gamma_{\mathrm{b}}}{\mathrm{m}}-\frac{0.0096}{\mathrm{~m}}+0.032
$$

The value of $\gamma_{\mathrm{b}}$ was calculated from Singamsetti and Wind (1980) (Equation 29).

69. Sallenger and Howd (1989) conducted field experiments in the vicinity of a longshore bar at Duck, North Carolina, in 1982 and 1985. They determined that wave energy became saturated at $\mathrm{H}_{\mathrm{rms}} / \mathrm{h}=0.32$ in the inner surf zone independent of $H_{o}$. However, all wave data were collected seaward of an inner bar, and measured values of $\mathrm{H}_{\mathrm{rms}} / \mathrm{h}$ included broken and unbroken waves.

70. Irregular wave decay models have been developed by Battjes and Janssen (1979), Dally (1980), and Thornton and Guza (1983) applying monochromatic decay models to a distribution of wave heights.

\section{Summary}

71. Several expressions and models have been developed for wave decay. This abbreviated and selective review of the considerable wave decay literature was made to illustrate the different methods used to predict wave decay. The methods may be complex and may attempt to physically explain energy dissipation in the surf zone, or they may be as simple as applying a constant multiplier to the water depth to estimate wave height. 


\section{Wave Runup}

72. Wave runup is defined as the elevation above SWL reached by an incident wave (Figure 8 ) and is a subject of great coastal engineering interest. For example, beach face change is directly controlled by the position of the water level (Larson and Kraus 1989), and the design height of coastal structures is determined by the amount of runup on the structure. The following paragraphs summarize selected equations developed from wave runup studies.

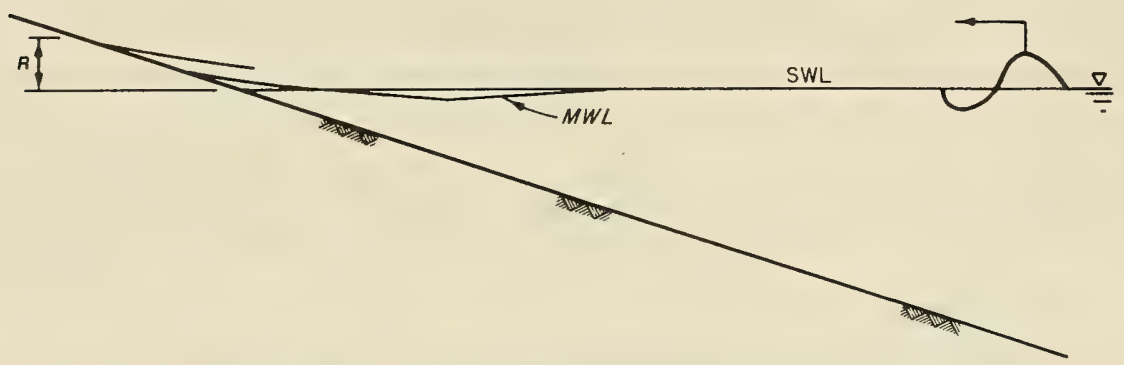

Figure 8. Definition sketch of wave runup

\section{Review}

73. Miche (1951) obtained an equation for runup $R$ normalized by deepwater wave height on a uniform slope:

$$
\frac{\mathrm{R}}{\mathrm{H}_{\mathrm{o}}}=\left(\frac{\pi}{2 \beta}\right)^{1 / 2}
$$

where $\beta$ is the beach slope in radians. Miche determined Equation 50 theoretically for linear standing (nonbreaking) waves on a slope.

74. Saville (1956) conducted laboratory experiments and measured runup of monochromatic waves on smooth-faced structures (structure slopes of 1/1.5, $1 / 2.25,1 / 3,1 / 4$, and $1 / 6$ ) on a $1 / 10$ plane slope. He also measured runup on $1 / 30$ and $1 / 10$ plane slopes. Saville combined the results with previously acquired data (Saville 1955) taken under conditions of $1 / 3$ and $1 / 1.5$ smoothfaced structures, $1 / 1.5$ step faced wall, $1 / 1.5$ riprap-faced wall, vertical wall, and recurved wall. The latter tests were also fronted by a $1 / 10$ plane 
slope. Saville developed often-used curves for $R / H_{0}$ as a function of wave steepness.

75. Hunt (1959) empirically determined an equation for runup from laboratory data as

$$
\frac{R}{H_{\circ}}=2.3 m\left(\frac{H_{\circ}}{T^{2}}\right)^{-1 / 2}
$$

where $R$ and $H_{0}$ are in feet. Battjes (1975) nondimensionalized the equation of Hunt to give runup as a function of the surf similarity parameter. The resulting equation was

$$
\frac{R}{H_{0}}=1.0 \xi_{0} \text { for } 0.1<\xi_{0}<2.3
$$

where $\xi_{0}$ is the offshore surf similarity parameter.

76. Ahrens and Titus (1985) found good agreement between Equation 52 and laboratory runup data collected by Saville (1956) and Savage (1958). The ratio of $\mathrm{R} / \mathrm{H}_{0}$ to $\xi_{0}$ was 0.967 for $\xi_{0} \leq 2.0$.

77. Ahrens (1981) empirically determined relationships for runup with irregular waves on smooth slopes based on data of van Oorschot and d'Angremond (1969), Kamphuis and Mohamed (1978), and Ahrens (1979). Runup was measured on structures ranging from $1 / 1$ to $1 / 4$. Ahrens found

$$
\begin{aligned}
& \frac{\mathrm{R}_{2}}{\mathrm{H}_{\mathrm{s}}}=1.61 \xi \\
& \frac{\mathrm{R}_{\mathrm{s}}}{\mathrm{H}_{\mathrm{s}}}=1.25 \xi \\
& \frac{\overline{\mathrm{R}}}{\mathrm{H}_{\mathrm{s}}}=0.84 \xi
\end{aligned}
$$


in which $R_{2}$ is 2-percent runup; the elevation exceeded by 2 percent of the total wave runup, $R_{S}$ is the significant runup; the average of the highest one-third of all runup, and $\bar{R}$ is the average runup. The surf similarity parameter was determined using $H_{s}$ and peak deepwater wavelength $\left(L_{p}\right) 。$, calculated using the peak period $T_{p}$ in Equation 1. Significant wave height was measured at the toe of the structures.

78. Mase and Iwagaki (1985) conducted laboratory experiments to estimate runup on plane slopes of $1 / 5,1 / 10,1 / 20$, and 1/30 with irregular waves that simulated a Pierson-Moskowitz spectrum. From the experimental results, the following equations were found:

$$
\begin{aligned}
& \frac{\mathrm{R}}{\left(\mathrm{H}_{\mathrm{s}}\right)_{0}}=\mathrm{a}(\tan \beta)^{\mathrm{b}}\left(\frac{\left(\mathrm{H}_{\mathrm{s}}\right)_{0}}{\left(\mathrm{~L}_{\mathrm{s}}\right)_{0}}\right)^{\mathrm{c}} \\
& \frac{\mathrm{R}}{\left(\mathrm{H}_{\mathrm{s}}\right)_{0}}=\mathrm{d}(\tan \beta)\left(\frac{\left(\mathrm{H}_{\mathrm{s}}\right)_{0}}{\left(\mathrm{~L}_{\mathrm{s}}\right)_{0}}\right)^{-\mathrm{e}}
\end{aligned}
$$

in which $\left(L_{s}\right)$ o is the significant deepwater wavelength, calculated using the significant period $T_{s}$ in Equation 1 , and the parameters a through $e$ varied depending on whether $\bar{R}, R_{\max }$, or $R_{s}$ was predicted.

\section{Summary}

79. The parameters used to determine runup on smooth plane slopes are beach slope and wave steepness. Factors that influence runup in nature also include the roughness and porosity of the slope, and corrections to the above equations must be made to account for the effect of these quantities.

\section{Analysis of Previous Data}

80. In the present study, selected data sets were reanalyzed to determine relationships for breaking wave height and plunge distance as a function of beach slope and deepwater wave steepness on plane sloping beaches. The following criteria were established to select data for this analysis:

a. The study was conducted on a fixed plane slope with monochromatic waves. 
b. Deepwater wave steepness was given, or wave height was measured in the horizontal section of the tank so that $H_{o} / L_{\circ}$ values could be calculated by linear wave theory.

c. No structures were present in the wave tank that would alter wave breaking or introduce reflections not associated with planar beaches.

The data sets selected are summarized in Table 3 , and the data used in the analysis are listed in Appendix A.

\section{Breaker depth index}

81. A breaker depth index was developed following the general (but not the particular) method of Weggel (1972) for 11 data sets for beach slopes covering $1 / 80$ to $1 / 10$. Breaker depth index was plotted versus deepwater wave steepness for each individual slope (Figure 9 (a-f)), and lines that visually best represented the data were drawn (dashed line). Calculated values, shown by solid lines, are explained below. The data were considerably scattered, and regression analysis could not be used. The data scatter apparently reflects inconsistencies of breakpoint location, breaker height, and/or water depth datum. Data collected on the 1/80 slope (Figure 9a) were scattered with no apparent trend; therefore, the average value of $\gamma_{b}$ was chosen for that slope.

82. The line slope $a(m)$ and zero intercept $b(m)$ of the best-fit lines from each slope were plotted versus beach slope (Figures 10 and 11). Equations were fit to $a(m)$ and $b(m)$ by using the method of least squares and combined to yield the following relationship for breaker depth index:

$$
\gamma_{b}=b(m)-a(m)\left(\frac{H_{0}}{L_{0}}\right)
$$

in which

$$
a(m)=5.00\left(1-e^{-43 m}\right)
$$

and

$$
b(m)=\frac{1.12}{\left(1+e^{-60 m}\right)}
$$

for $0.0007 \leq \mathrm{H}_{\mathrm{o}} / \mathrm{L}_{\mathrm{o}} \leq 0.0921$ and $1 / 80 \leq \mathrm{m} \leq 1 / 10$. Equation 58 is presented in Figure 12 for beach slopes ranging from $1 / 80$ to $1 / 10$ and also in Figure 9 
Table 3

Data Summary for Breaker Index and

Plunge Distance on Plane S1opes

\begin{tabular}{|c|c|c|c|}
\hline Source & Breaker data & Plunge data & Slope \\
\hline Iversen (1952) & $\mathrm{X}^{*}$ & - & $\begin{array}{l}1 / 50 \\
1 / 30 \\
1 / 20 \\
1 / 10\end{array}$ \\
\hline $\begin{array}{l}\text { Horikawa } \\
\text { and Kuo (1967) }\end{array}$ & $\mathrm{X}$ & -- & $\begin{array}{l}1 / 80 \\
1 / 30 \\
1 / 20\end{array}$ \\
\hline Galvin (1969) & $\mathrm{X}$ & $\mathrm{x}$ & $\begin{array}{l}1 / 20 \\
1 / 10\end{array}$ \\
\hline $\begin{array}{l}\text { Saeki and } \\
\text { Sasaki (1973) }\end{array}$ & $\mathrm{X}$ & - & $1 / 50$ \\
\hline $\begin{array}{l}\text { Iwagaki et al. } \\
\text { (1974) }\end{array}$ & $\mathrm{X}$ & - & $\begin{array}{l}1 / 30 \\
1 / 20 \\
1 / 10\end{array}$ \\
\hline Walker (1974b) & $\mathrm{x}$ & - & $1 / 30$ \\
\hline $\begin{array}{l}\text { Singamsetti and } \\
\text { Wind (1980) }\end{array}$ & $\mathrm{X}$ & $\mathrm{X}$ & $\begin{array}{l}1 / 40 \\
1 / 20 \\
1 / 10\end{array}$ \\
\hline Mizuguchi (1981) & $\mathrm{X}$ & - & $1 / 10$ \\
\hline $\begin{array}{l}\text { Maruyama et a1. } \\
\text { (1983) }\end{array}$ & $\mathrm{X}$ & -- & $1 / 29.4$ \\
\hline Visser (1982) & $\mathrm{X}$ & $\mathrm{X}$ & $\begin{array}{l}1 / 20 \\
1 / 10\end{array}$ \\
\hline Stive (1985) & $\mathrm{X}$ & - & $1 / 40$ \\
\hline Present Study & $\mathrm{X}$ & $\mathrm{X}$ & $1 / 30$ \\
\hline
\end{tabular}

* Symbol X indicates quantity available. 


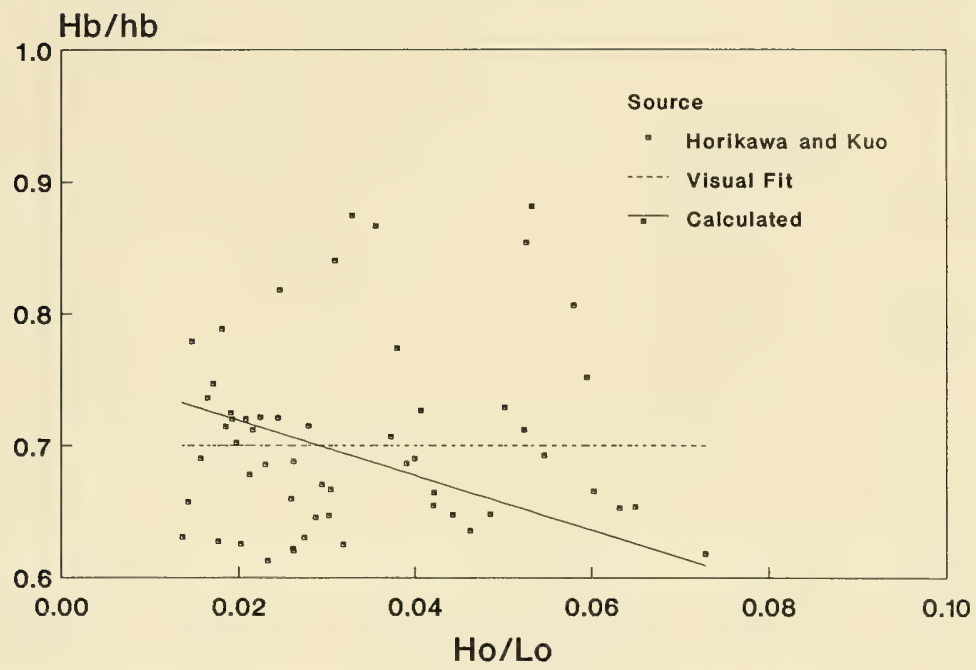

a. $\mathrm{m}=1 / 80$

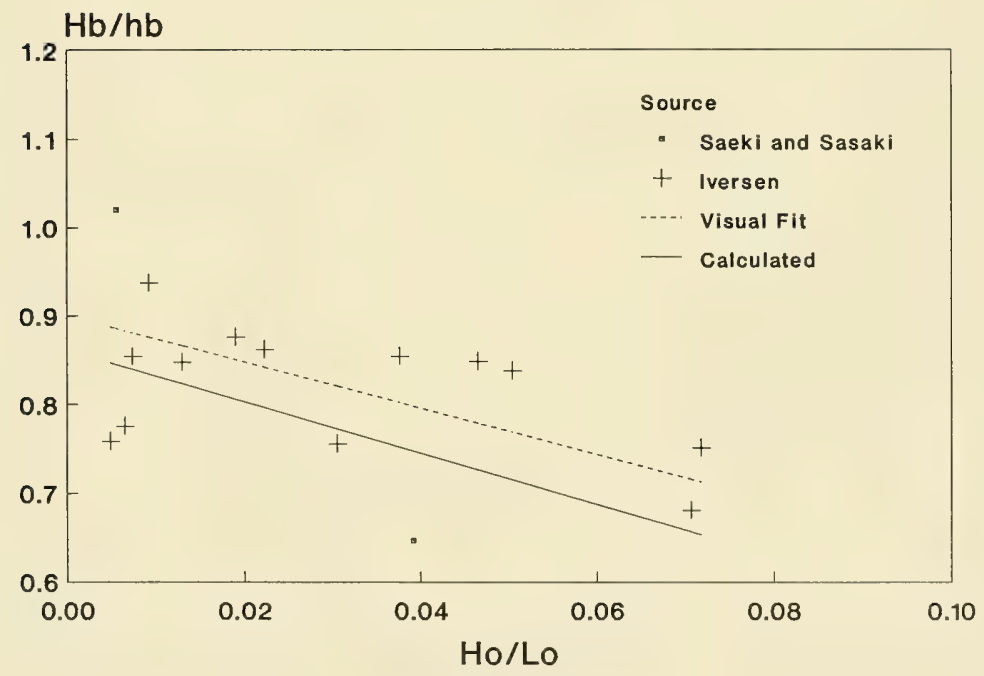

b. $\mathrm{m}=1 / 50$

Figure 9. Breaker depth index as a function of $H_{0} / L_{0}$ (Sheet 1 of 3 ) 


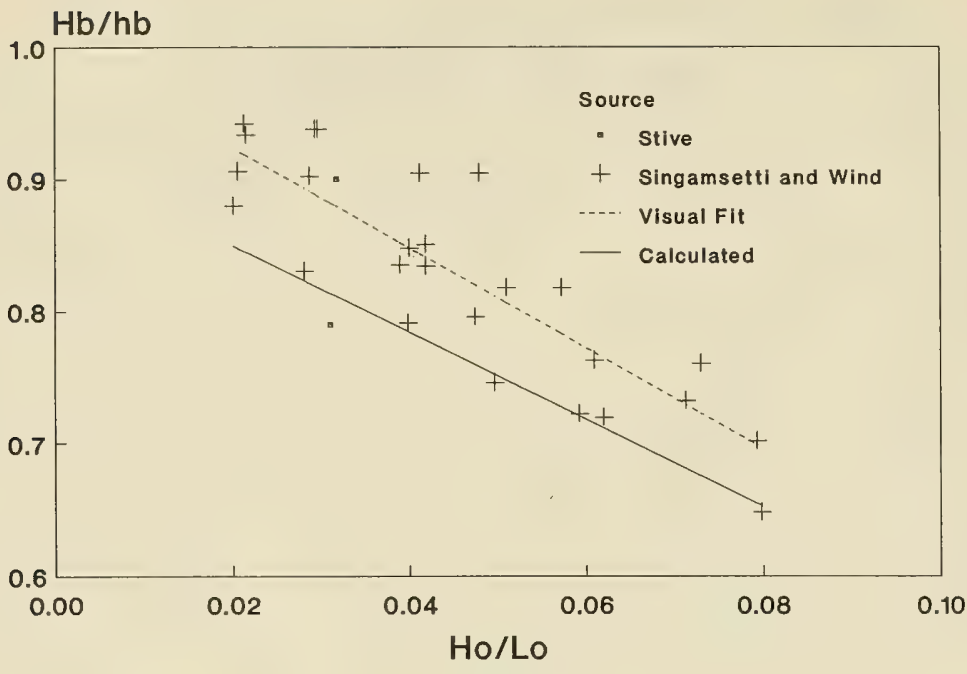

c. $\mathrm{m}=1 / 40$

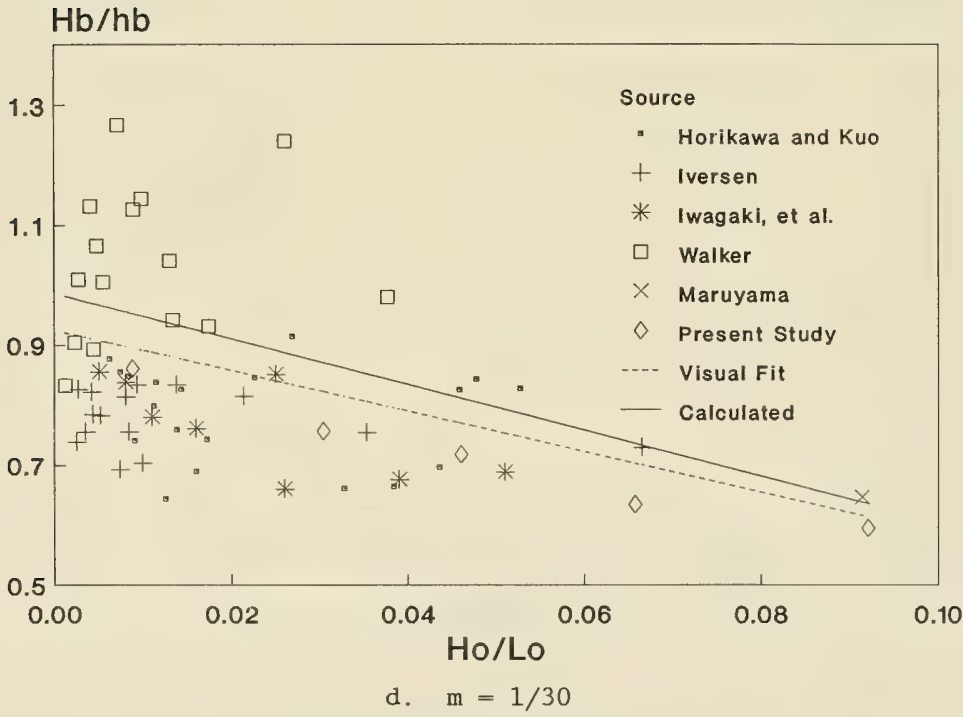

Figure 9. (Sheet 2 of 3 ) 

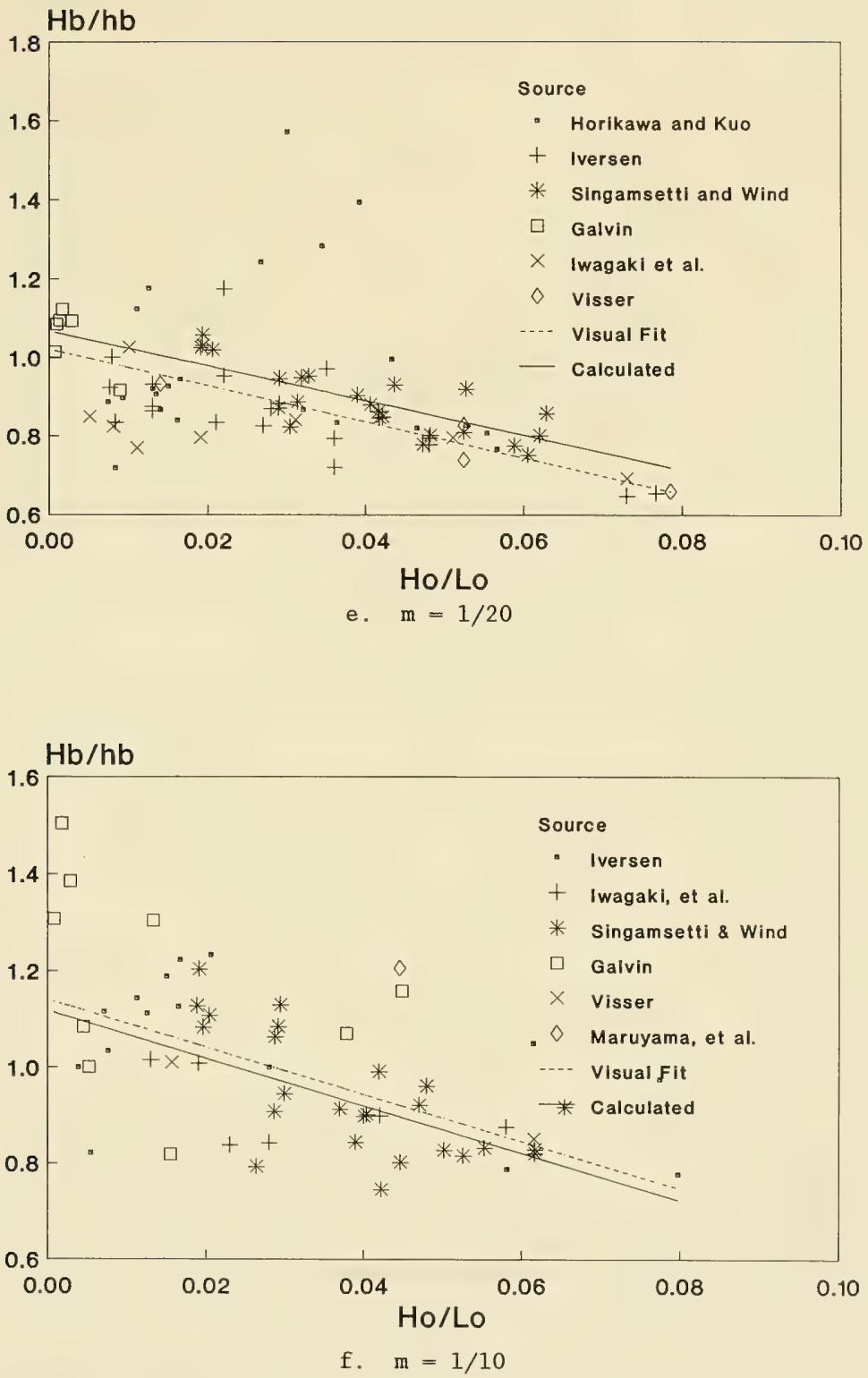

Figure 9. (Sheet 3 of 3 ) 


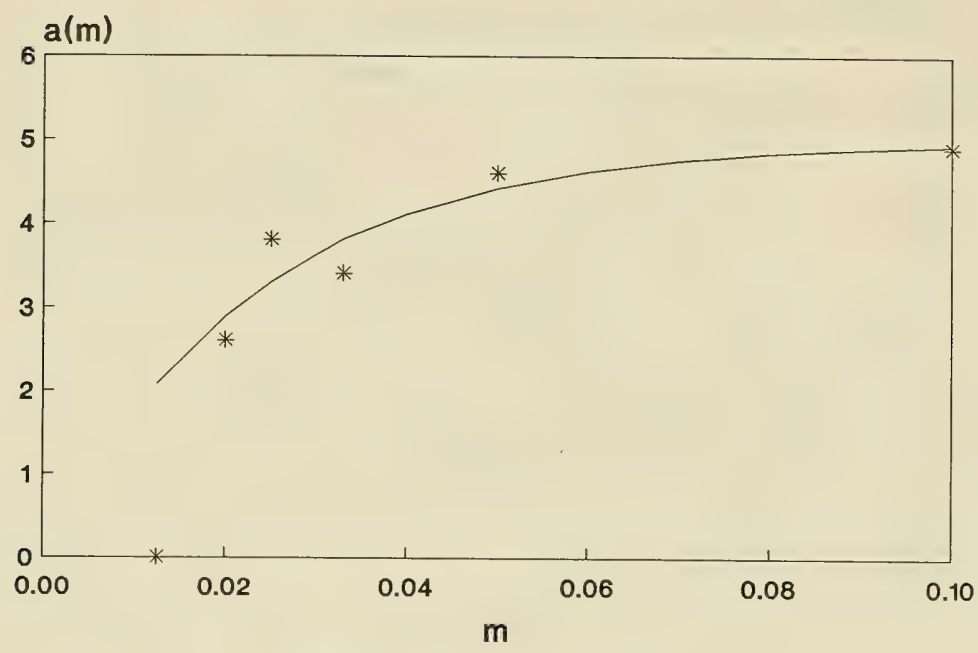

Figure 10. Function a(m)

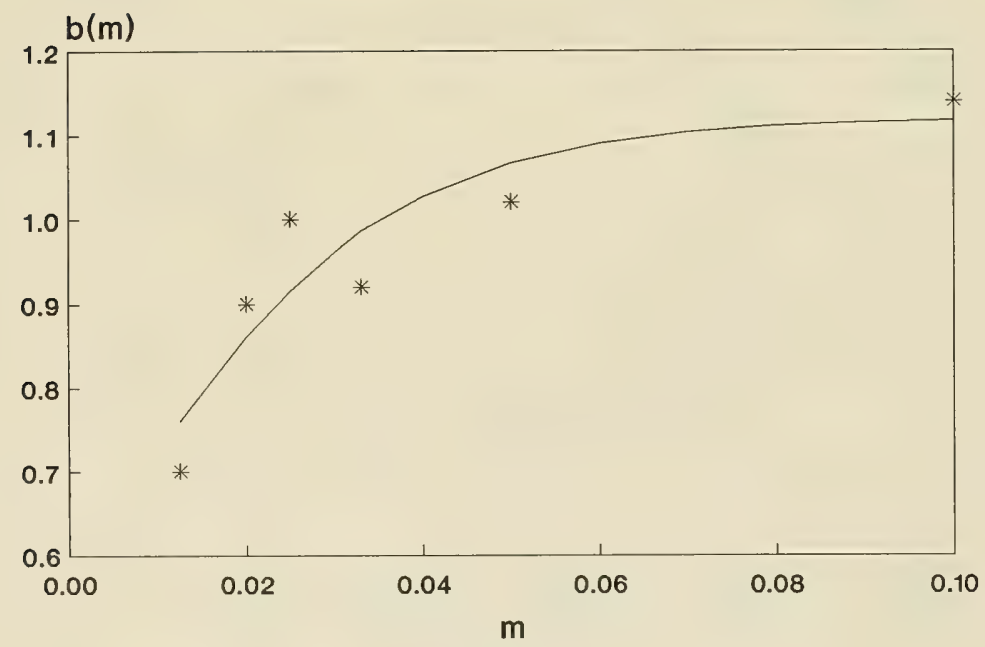

Figure 11. Function $b(\mathrm{~m})$ 


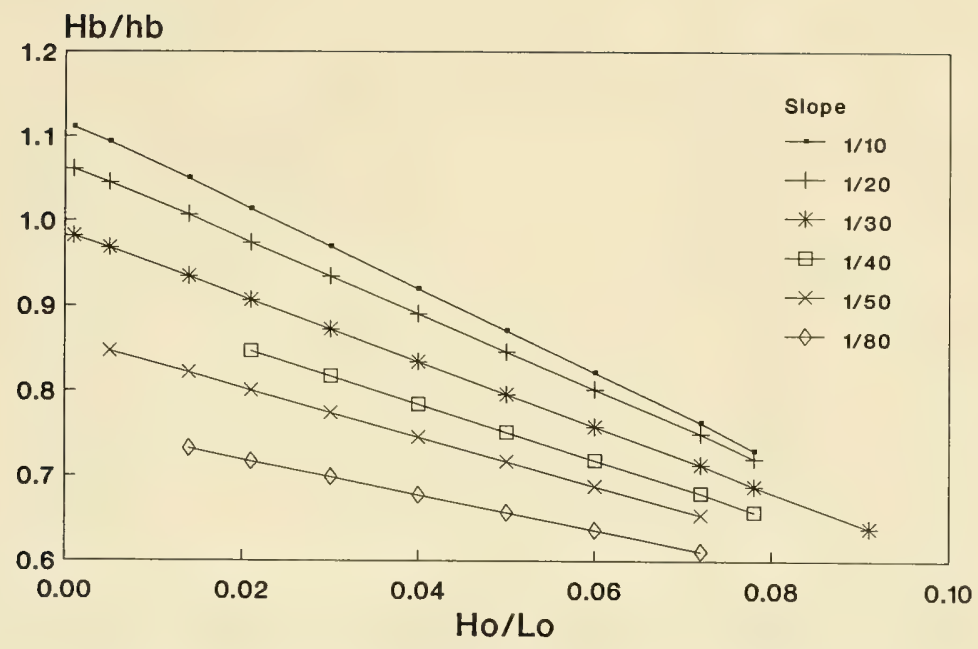

Figure 12. Calculated values of $\gamma_{b}$ for plane slopes as a function of $\mathrm{H}_{0} / \mathrm{L}_{\mathrm{o}}$

(a-f) for each beach slope, represented by the solid line.

\section{Breaker height index}

83. Breaker height index was plotted as a function of $H_{o} / L_{o}$ for each beach slope (Figure $13(\mathrm{a}-\mathrm{f})$ ). A power law regression of $\Omega_{b}$ as a function of $\mathrm{H}_{\mathrm{o}} / \mathrm{L}_{\mathrm{o}}$ was made for each slope and is represented by the dashed line in Figure 13 (a-f). The coefficient $\mathrm{C}(\mathrm{m})$ and exponent $\mathrm{n}(\mathrm{m})$ values obtained from each regression were plotted versus beach slope in Figures 14 and 15 , respectively. The coefficients and exponents for the $1 / 80$ slope and $1 / 40$ slope do not follow the trend of the other data points. The majority of data collected on the $1 / 40$ slope are from one source (Singamsetti and Wind 1980), and all data collected on the 1/80 slope are from Horikawa and Kuo (1967). Singamsetti and Wind may have defined the breaking wave height and/or break point differently than authors of other data sources. The 1/80 slope experiment had a concrete bottom and was performed in a different tank from the other experiments of Horikawa and Kuo (1967), which had a steel plate bottom.* Since $\mathrm{C}(\mathrm{m})$ and $\mathrm{n}(\mathrm{m})$ for slopes of $1 / 80$ and 1/40 did not follow

* Personal Communication, 1986, Kiyoshi Horikawa, Professor, Department of Civil Engineering, University of Tokyo, Tokyo, Japan. 


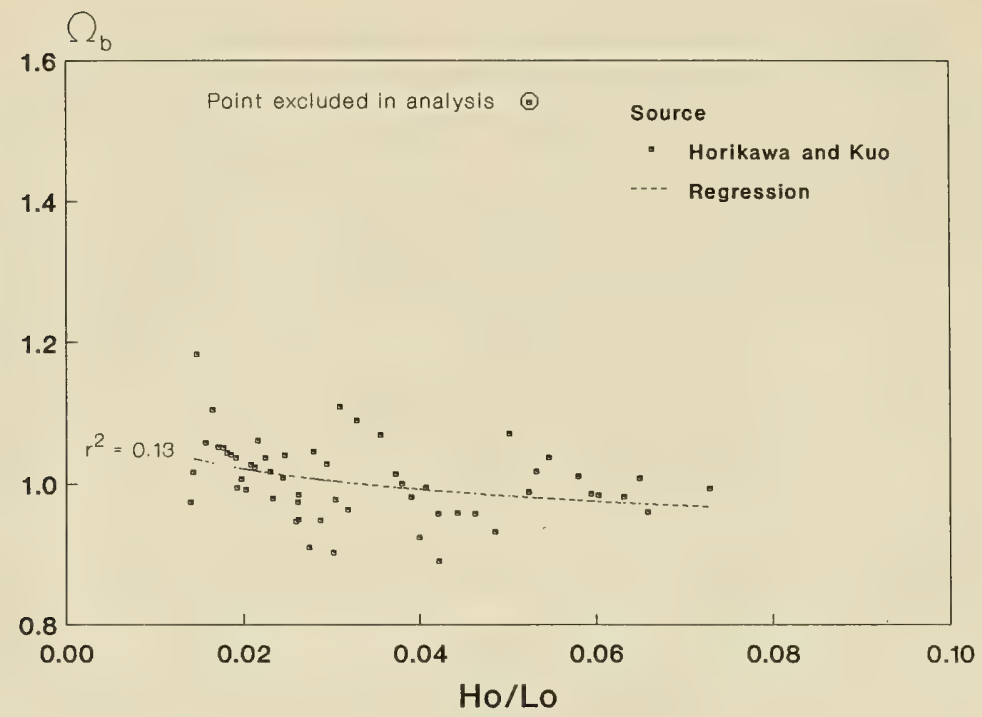

a. $\mathrm{m}=1 / 80$

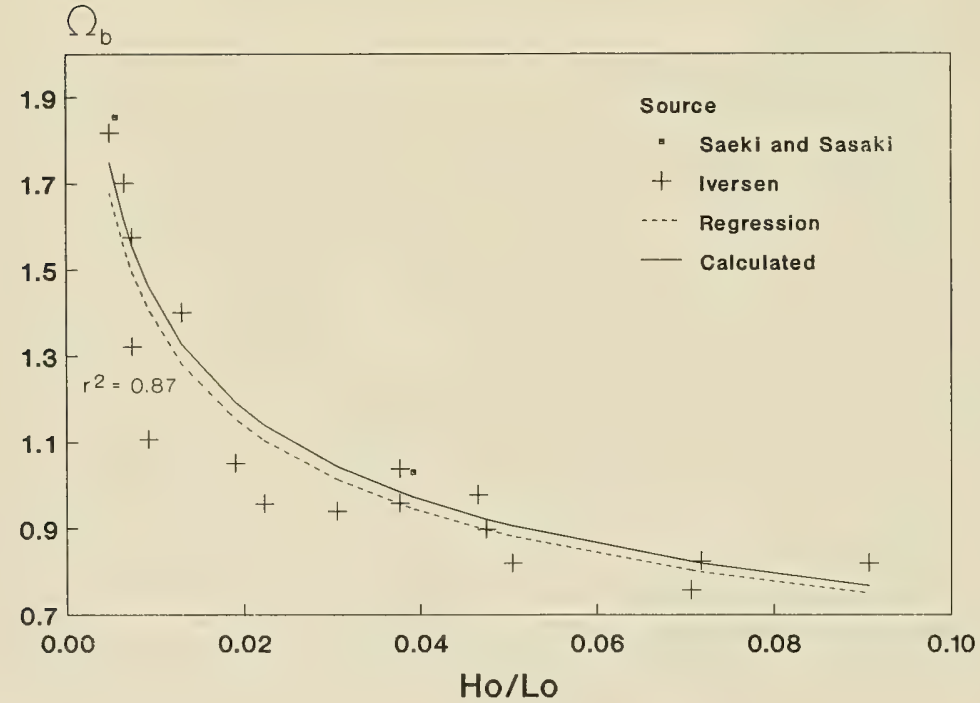

b. $m=1 / 50$

Figure 13. Breaker height index as a function of $H_{\circ} / L_{\circ}$ (Sheet 1 of 3 ) 

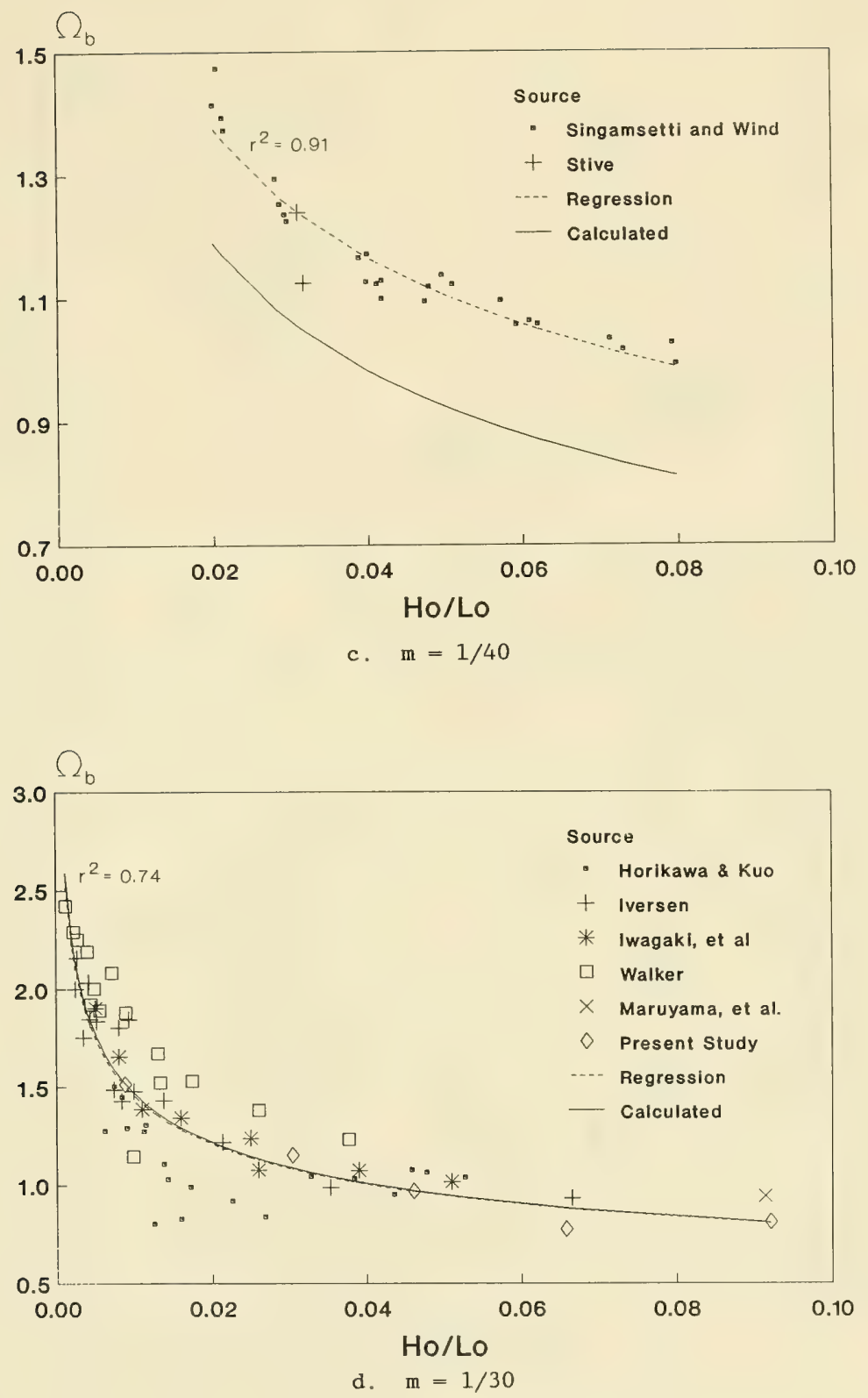

Figure 13. (Sheet 2 of 3 ) 


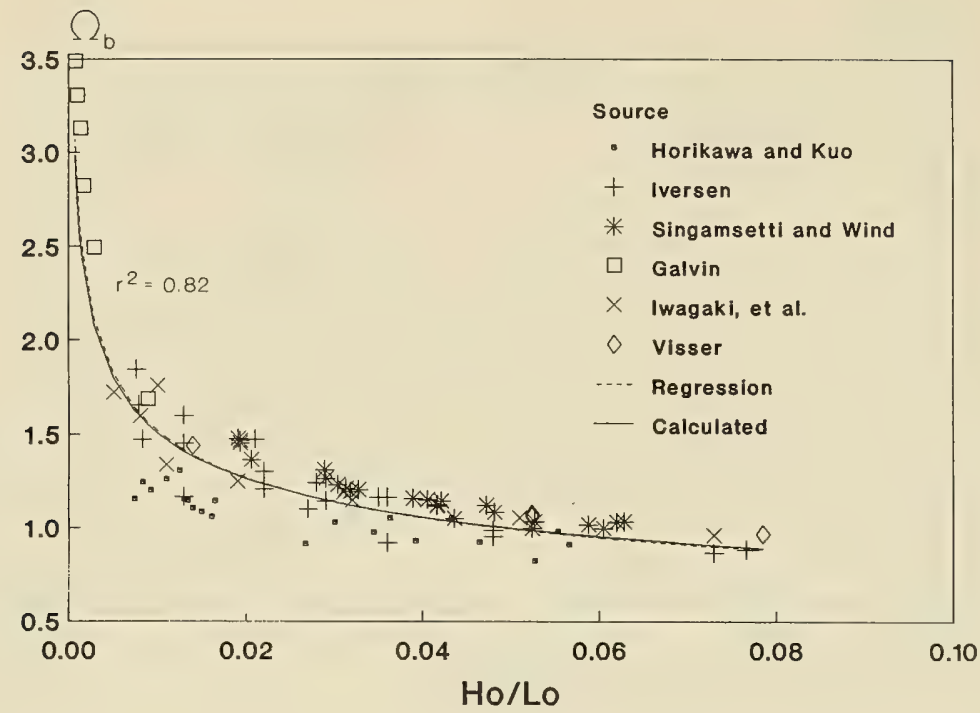

e. $\mathrm{m}=1 / 20$

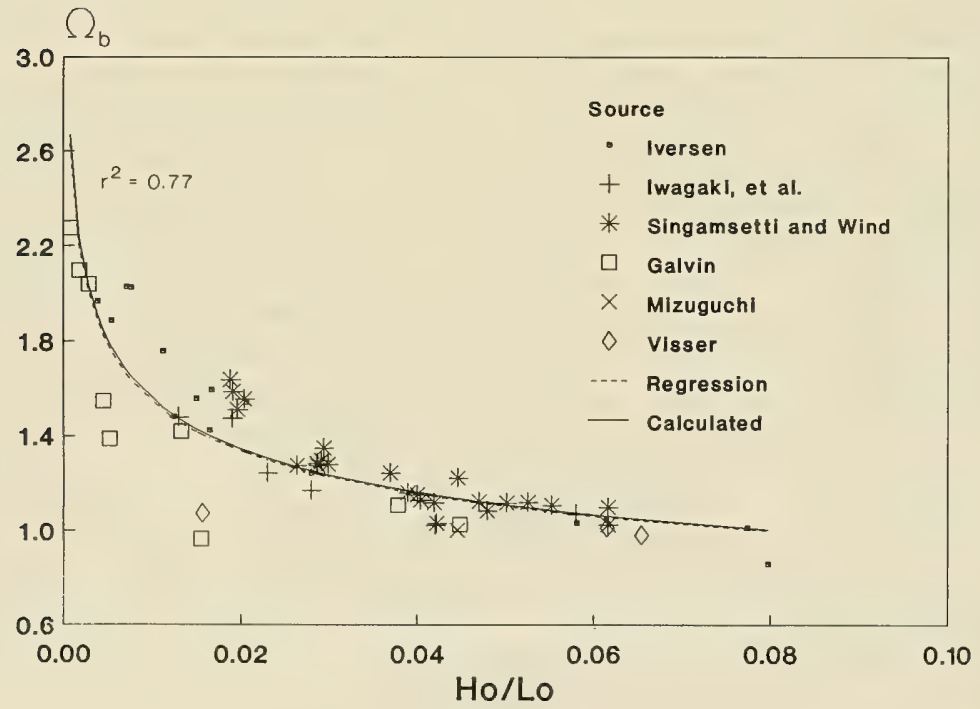

f. $m=1 / 10$

Figure 13. (Sheet 3 of 3 ) 


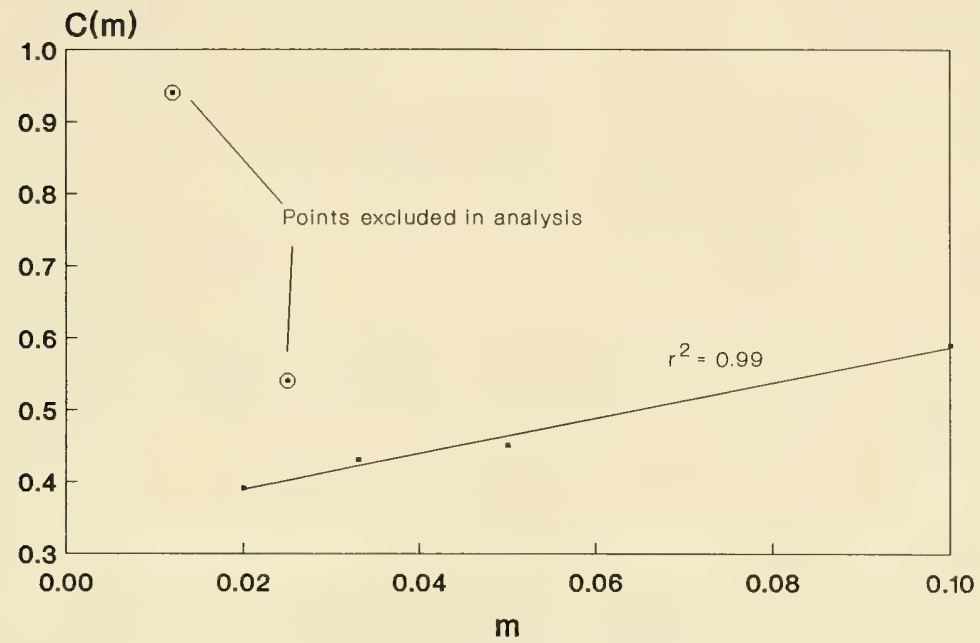

Figure 14. Function C(m)

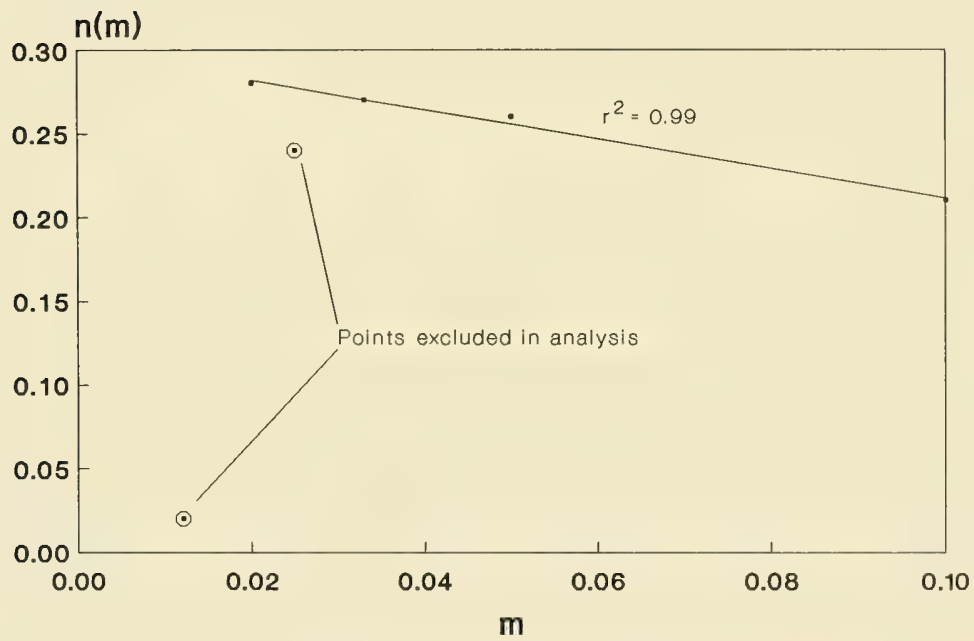

Figure 15. Function $\mathrm{n}(\mathrm{m})$ 
the trend of the other coefficients, linear regressions were made on $\mathrm{C}(\mathrm{m})$ and $\mathrm{n}(\mathrm{m})$ for values obtained only on $1 / 50,1 / 30,1 / 20$, and $1 / 10$ slopes. The resulting equation for $\Omega_{b}$ is :

$$
\Omega_{\mathrm{b}}=\mathrm{C}(\mathrm{m})\left(\frac{\mathrm{H}_{\mathrm{o}}}{\mathrm{L}_{\mathrm{o}}}\right)^{-\mathrm{n}(\mathrm{m})}
$$

in which

$$
C(m)=0.34+2.47 m
$$

and

$$
\mathrm{n}(\mathrm{m})=0.30-0.88 \mathrm{~m}
$$

for $1 / 50 \leq m \leq 1 / 10$ and $0.007 \leq H_{0} / L_{0} \leq 0.0921$. The solid line in Figure $13(\mathrm{~b}-\mathrm{f})$ represents Equation 61 . The equation shows good agreement with the regression analysis for beach slopes of $1 / 10,1 / 20,1 / 30,1 / 50$. Equation 61 underpredicts $\Omega_{\mathrm{b}}$ for the $1 / 40$ slope data, since $\mathrm{C}(1 / 40)$ was much higher than the other $\mathrm{C}(\mathrm{m})$ values and not included in analysis.

84. Overall, Equation 61 predicts breaker height index we1l, and it also gives reasonable values compared with other breaker height equations (Figure $6(b-d))$.

\section{Plunge distance}

85. Plunge distance data were available from three sources covering slopes ranging from $1 / 5$ to $1 / 40$. Figure 16 shows a comparison of the data set with Equation 36 (Galvin 1969). The trend in the data is underpredicted by Equation 36 on all slopes except those pertaining to the Galvin experiment. The large variation between maximum and minimum values at each slope indicates plunge distance is not solely a function of beach slope. Plunge distance normalized by breaking wave height was plotted versus the offshore surf similarity parameter (Figure 17). The solid line in Figure 17 was visually fit to the data and represents the following relationship 


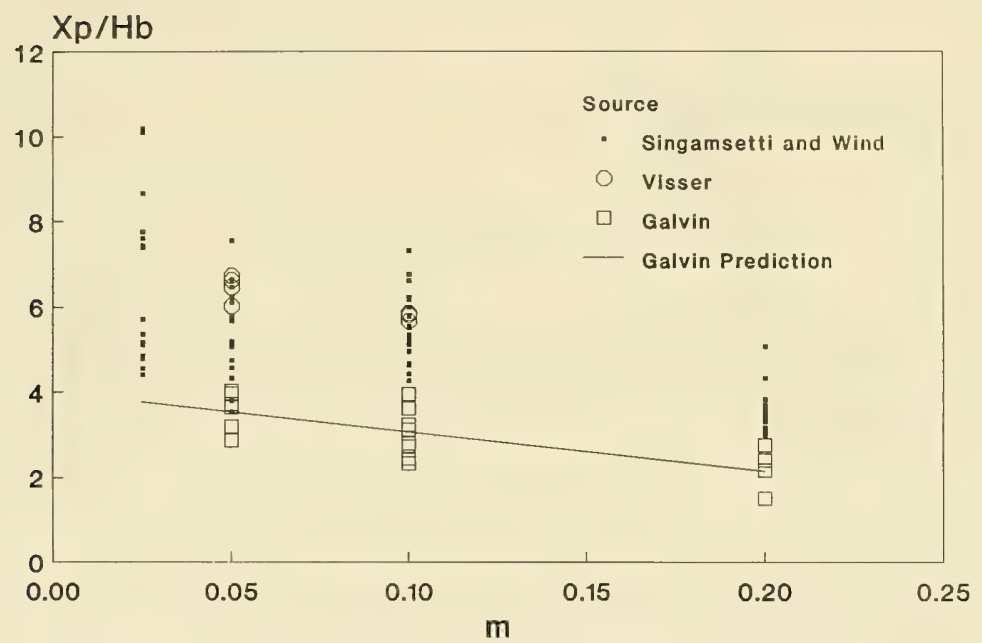

Figure 16. Comparison of measured plunge distance and empirical prediction of Galvin (1969)

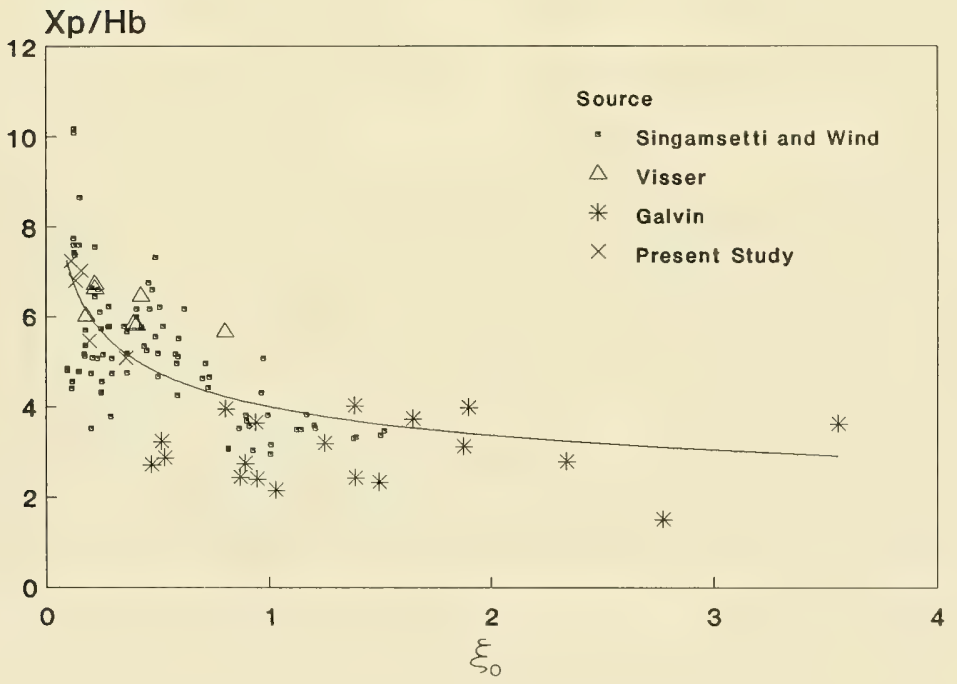

Figure 17. $\mathrm{X}_{\mathrm{p}} / \mathrm{H}_{\mathrm{b}}$ as a function of $\xi_{\mathrm{o}}$ 


$$
\frac{X_{p}}{H_{b}}=4.0 \xi_{0}^{-0.25}
$$

which also can be written as

$$
\frac{\mathrm{X}_{\mathrm{p}}}{\mathrm{H}_{\mathrm{b}}}=4.0 \mathrm{~m}^{-1 / 4}\left(\frac{\mathrm{H}_{\mathrm{o}}}{\mathrm{L}_{\mathrm{o}}}\right)^{1 / 8}
$$

86. Plunge distance is also expressed as a function of the local surf similarity parameter by

$$
\frac{\mathrm{X}_{\mathrm{p}}}{\mathrm{H}_{\mathrm{b}}}=3.4 \xi_{\mathrm{b}}^{-0.20}
$$

shown in Figure 18. Equation 66 was also obtained visually.

87. Plunge distance normalized by deepwater wave height was compared with $\xi_{0}$ (Figure 19). The best visual fit equation is

$$
\frac{X_{p}}{H_{o}}=4.25 \xi_{0}^{-0.36}
$$

88. Figures 17-19 all show scatter in the data, but plunge distance normalized by $\mathrm{H}_{\circ}$ shows considerably more scatter. The high $\mathrm{X}_{\mathrm{p}} / \mathrm{H}_{\mathrm{o}}-\mathrm{values}$ indicated on Figure 19 were all from the Galvin (1969) data set and from tests in which he observed multiple wave forms, or solitons, present in the tank. The subject of solitons will be discussed further in Part III. However, the effect of solitons is not apparent if $x_{p}$ is normalized by breaking wave height. Therefore, based on the data set, plunge distance normalized by breaking wave height should be used to determine plunge distance, and data are least scattered if $\mathrm{X}_{\mathrm{p}} / \mathrm{H}_{\mathrm{b}}$ is plotted as a function of the offshore surf similarity parameter. 


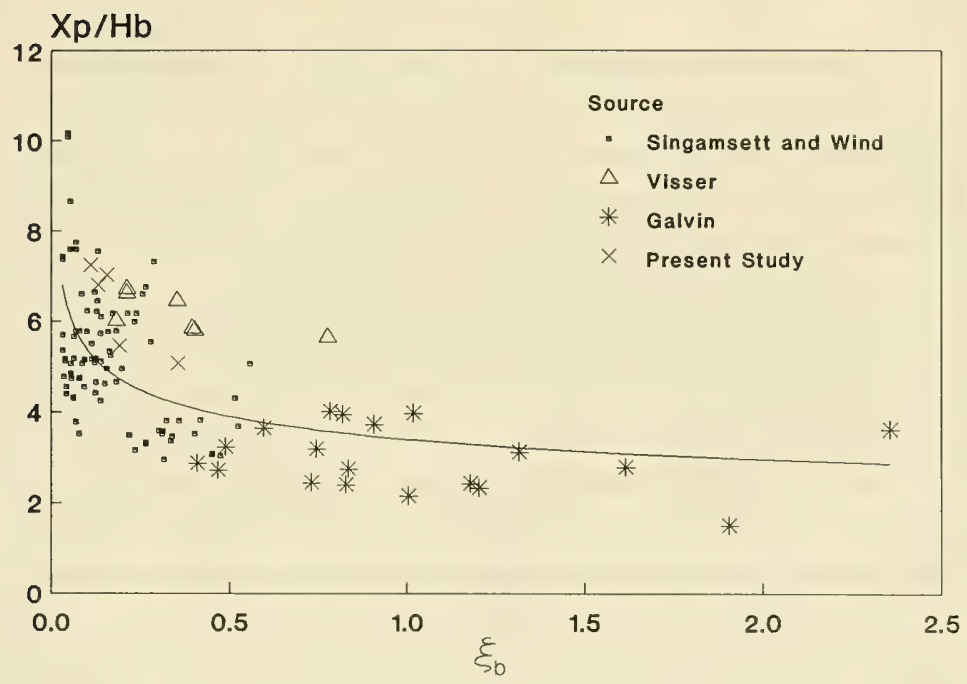

Figure 18. $\mathrm{X}_{\mathrm{p}} / \mathrm{H}_{\mathrm{b}}$ as a function of $\xi_{\mathrm{b}}$

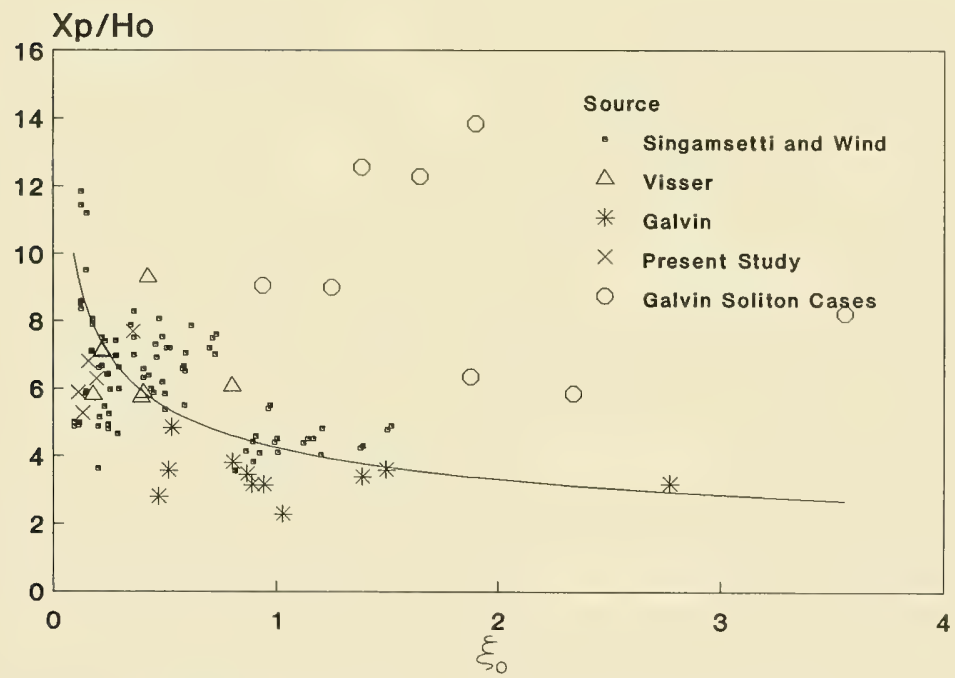

Figure 19. $\mathrm{X}_{\mathrm{p}} / \mathrm{H}_{\circ}$ as a function of $\xi_{0}$ 
89. The literature and equations cited in this review provide a necessary base for comparing wave breaking properties on plane slopes (previous studies) to barred profiles (present study). More than one relationship is usually given for each property, but most equations illustrate that beach slope and deepwater wave steepness describe breaking wave characteristics. 90. Equations were developed for breaker depth index, breaker height index, and plunge distance as a function of beach slope and deepwater wave steepness. Data used to determine the empirical equations were based on data acquired on plane slopes from previous studies and the present study. The data were scattered and equations were determined from visual fit of the data for breaker depth index and plunge distance. The data were better behaved for breaker height index than for breaker depth index and plunge distance, and a power law regression was used to determine this index. 


\section{PART III: EXPERIMENT ARRANGEMENT}

\section{Facility and Equipment}

91. Tests were conducted in a 150 -ft*-long, 1.5-ft-wide, and 3-ft-high glass-walled tank (Figure 20). The tank contained a 1/30 smooth concretecapped slope that began $69 \mathrm{ft}$ from the generator board. The section between the slope and the generator was horizontal. Waves were generated by an electronically controlled hydraulic system that drove a piston-type wave board. Displacement of the wave board was controlled by a command signal transmitted to the board by a synthesized function generator for monochromatic waves and by a microcomputer for irregular waves. The microcomputer can also produce monochromatic wave signals for an operator-specified length of time. Although the synthesized function generator can only send monochromatic wave signals, it is independent of the microcomputer and operates until it is manually turned off. The synthesized function generator was more convenient to use if data collection was not necessary, such as when visual observations were made.

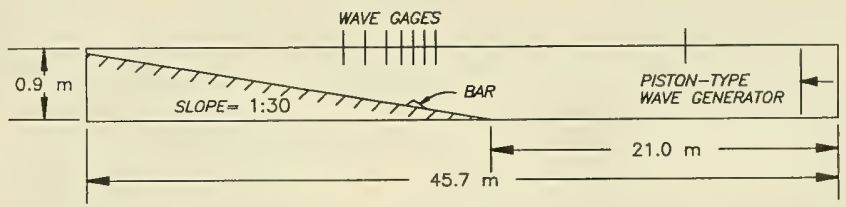

DISTORTED SCALE, $1 \mathrm{H}=5 \mathrm{~V}$ TANK WIDTH $=0.46 \mathrm{~m}$

Figure 20. Sketch of tank used in study

92. The water surface elevation was measured with eight double-wire resistance-type gages connected to the microcomputer by cables. The gages were calibrated each day prior to testing. Gage 1 was located $30 \mathrm{ft}$ from the wave generator. Gages 2 and 3 were placed "seaward" of the bar and positioned to measure wave reflection using the method described by Goda and Suzuki (1977). Gage 4 was placed near the point of incipient wave breaking. Gages 5 through 8 were distributed through the surf zone and used to measure wave

* A table of factors for converting non-SI units of measurement to SI (metric) units is presented on page 8 . 
heights shoreward of breaking. Locations of Gages 2 through 8 were dependent upon bar location and varied from test to test. The distance between Gages 2 and 3 was dependent upon wave period and water depth, and this distance also varied from test to test. A photograph of Gages 3 through 6 is shown in Figure 21. Wave data were analyzed using the Time Series Analysis (TSA) program*. The TSA program was developed at the Coastal Engineering Research Center (CERC) to provide several analyses of the wave record at each gage. The program was used in the present study to perform downcrossing analysis to obtain average wave height $H$, significant wave height $H_{s}$, maximum wave height $H_{\max }$, average wave period $T$, significant period $T_{s}$, and average free-surface water elevation $\eta$. The program was also used to make a reflection analysis as described by Goda and Suzuki (1977) and execute a singlechannel frequency analysis to obtain peak period $T_{p}$ at each gage.

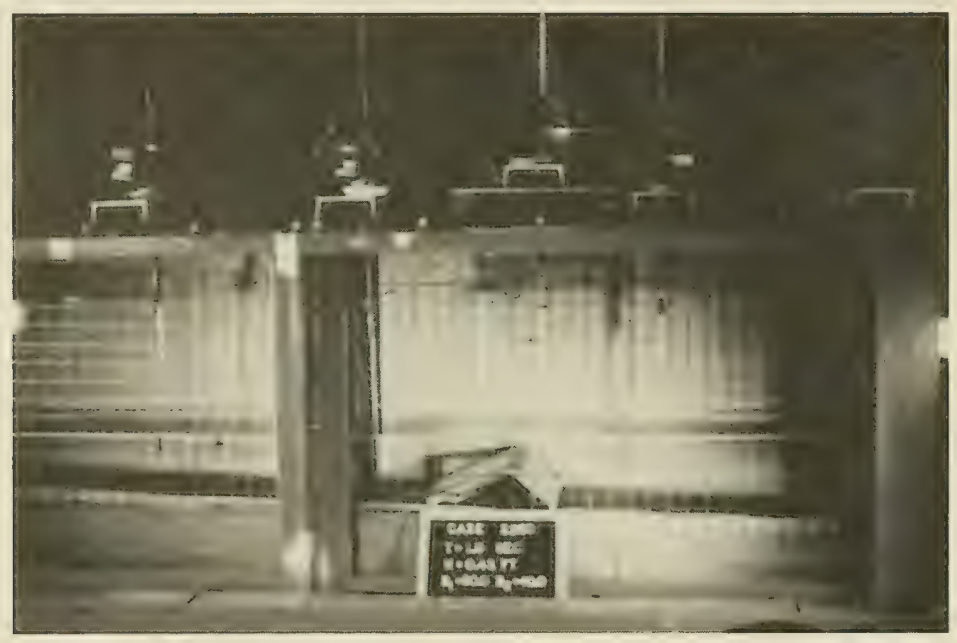

Figure 21. Gages 3-6

93. Two Sony 3/4-in. video cameras mounted on tripods were arranged to focus on the vicinity of wave breaking in the tests: one recorded waves at the break point, and the other recorded the plunge and splash distances. A

* C. E. Long, 1985, "Time Series Analysis," Unpublished Computer Program, US Army Engineer Waterways Experiment Station, Coastal Engineering Research Center, Vicksburg, MS. 
grid spaced 2 by 2 in. was taped on the front glass wall of the tank (nearest to cameras) for a reference in analysis of the videotapes. White paper was taped to the back glass wall of the tank to enhance visibility of the recorded waves. A stopwatch was placed in the viewing area of each camera to synchronize review of the two videos. A photograph of the video equipment is shown in Figure 22.

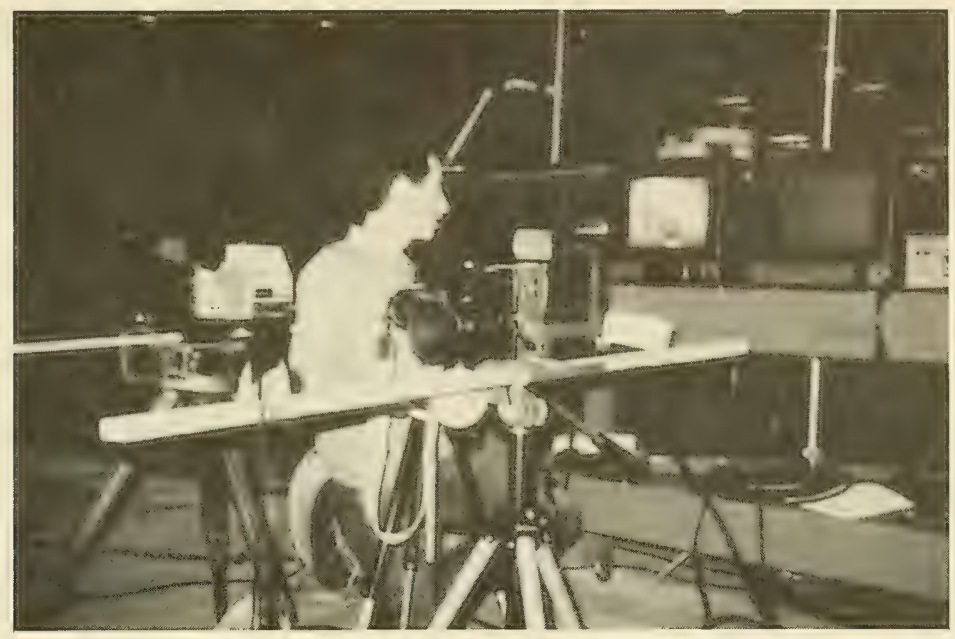

Figure 22. Video equipment used in study

94. A 35-mm camera provided general documentation for each test. Two still photographs were taken of the bar in the tank, and approximately ten photographs were taken of waves transforming over the structure.

95. Bars were constructed of 3/4-in. marine plywood. A schematic of a typical bar is shown in Figure 23. The seaward and shoreward faces of the structure were connected with strap hinges, and the two ends were anchored at the correct length with predrilled 1/4-in. steel bars. Observations during preliminary tests showed that sections of longer bars flexed under the waves. To minimize this condition, the longer structures were supported by legs attached underneath to prevent flexing or "breathing" of the seaward and shoreward faces due to wave action. Styrofoam was placed in the opening at the crest and taped in position to maintain a flush surface. Styrofoam also was used to seal the sides of the bar against the walls of the tank. Prior to 


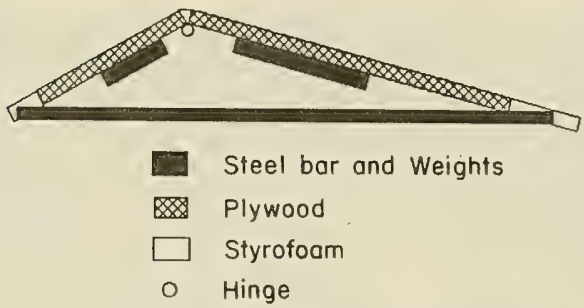

Figure 23. Definition sketch of typical bar

installation, steel plates were attached underneath the structure to prevent it from floating or moving when subjected to waves.

\section{Design Wave Conditions}

96. The deepwater wave steepness in part controls the breaking wave characteristics (Part II). Therefore, it was desirable to generate waves with a broad range of deepwater wave steepnesses. To determine the wave generating characteristics of the tank, maximum wave heights were produced and measured in the horizontal section over a range of periods from 0.7 to 7.0 sec at a fixed water depth. A water depth of $1.25 \mathrm{ft}$ provided the best location in the tank for video taping of the wave breaking process and was maintained for all tests. This water depth also allowed generation of relatively high waves for improved accuracy with videotape analysis and minimized the effect of surface tension. Maximum deepwater wave steepness $H_{0} / L_{0}$ for each wave period was determined by calculating $\mathrm{H}_{\mathrm{o}}$ and $\mathrm{L}_{\mathrm{o}}$ from linear wave theory. For waves normally incident over the bottom contours with no losses or input of energy, the expression for deepwater wave height is 


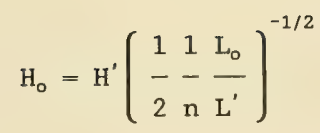

in which $\mathrm{n}$ is

$$
\mathrm{n}=\frac{1}{2}\left(\frac{2 \mathrm{kh}}{\sinh (2 \mathrm{kh})}\right)
$$

and $H^{\prime}$ and $L^{\prime}$ are the wave height and wavelength, respectively, in the horizontal section of the tank ( $h=1.25 \mathrm{ft}$ ), and $k=2 \pi / L$ is the wave number. The wavelength at a depth $h$ can be calculated by linear wave theory as :

$$
\mathrm{L}=\mathrm{L}_{\mathrm{o}} \tanh (\mathrm{kh})
$$

The observed maximum wave heights and resulting deepwater wave steepnesses are tabulated in Table 4. Maximum $H_{0} / L_{0}$ is plotted versus period in Figure 24. A range of wave conditions based on deepwater wave steepness was selected from Figure 24 and is listed in Table 5.

\begin{tabular}{|c|c|c|c|c|c|}
\hline $\begin{array}{l}\mathrm{h} \\
\mathrm{ft}\end{array}$ & $\begin{array}{c}\mathrm{T} \\
\mathrm{sec}\end{array}$ & $\begin{array}{c}\mathrm{H}_{\max } \\
\mathrm{ft}\end{array}$ & $\begin{array}{l}\mathrm{L}_{\mathrm{o}} \\
\mathrm{ft}\end{array}$ & $\begin{array}{l}\mathrm{H}_{\mathrm{o}} \\
\mathrm{ft}\end{array}$ & $\mathrm{H}_{\mathrm{o}} / \mathrm{L}_{\mathrm{o}}$ \\
\hline 1.25 & 0.7 & 0.21 & 2.51 & 0.21 & 0.0844 \\
\hline 1.25 & 1.0 & 0.46 & 5.12 & 0.49 & 0.0965 \\
\hline 1.25 & 2.0 & 0.58 & 20.50 & 0.59 & 0.0286 \\
\hline 1.25 & 3.0 & 0.92 & 46.12 & 0.80 & 0.0174 \\
\hline 1.25 & 4.0 & 0.67 & 82.00 & 0.51 & 0.0063 \\
\hline 1.25 & 5.0 & 0.42 & 128.12 & 0.29 & 0.0023 \\
\hline 1.25 & 6.0 & 0.29 & 184.49 & 0.18 & 0.0010 \\
\hline 1.25 & 7.0 & 0.25 & 251.11 & 0.15 & 0.0006 \\
\hline
\end{tabular}

Table 4 Maximum Wave Height Observed in the 18-In. Tank 
18-in TANK $(h=1.25 \mathrm{ft})$

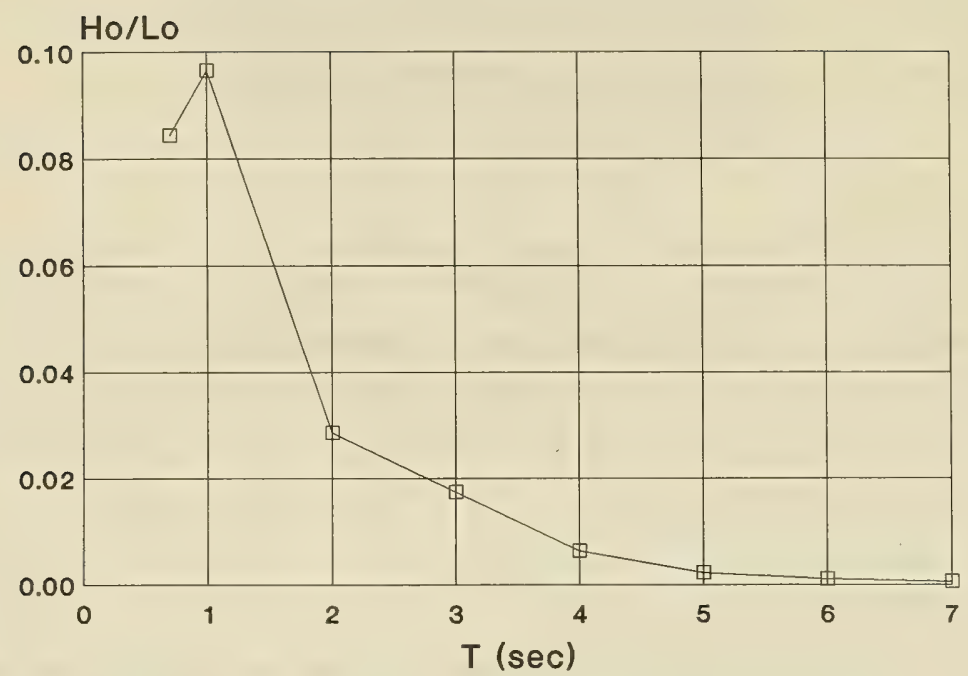

Figure 24. Maximum $H_{0} / L_{0}$ as a function of $T$

Table 5

Range of Wave Conditions

\begin{tabular}{|c|c|c|c|c|c|c|}
\hline $\begin{array}{c}\text { Wave } \\
\text { Condition }\end{array}$ & $\begin{array}{c}\mathrm{T} \\
\mathrm{sec}\end{array}$ & $\mathrm{H}_{\mathrm{o}} / \mathrm{L}_{\mathrm{o}}$ & $\begin{array}{l}\mathrm{L}_{\mathrm{o}} \\
\mathrm{ft}\end{array}$ & $\begin{array}{l}\mathrm{H}_{\mathrm{o}} \\
\mathrm{ft}\end{array}$ & $\begin{array}{l}\mathrm{L}^{\prime} \\
\mathrm{ft}\end{array}$ & $\begin{array}{l}\mathrm{H}^{\prime} \\
\mathrm{ft}\end{array}$ \\
\hline 1 & 1.00 & 0.0965 & 5.12 & 0.49 & 4.76 & 0.46 \\
\hline 2 & 1.00 & 0.090 & 5.12 & 0.46 & 4.76 & 0.43 \\
\hline 3 & 0.70 & 0.080 & 2.51 & 0.20 & 2.50 & 0.20 \\
\hline 4 & 1.00 & 0.070 & 5.12 & 0.36 & 4.76 & 0.33 \\
\hline 5 & 1.25 & 0.060 & 8.01 & 0.48 & 6.64 & 0.44 \\
\hline 6 & 1.50 & 0.050 & 11.53 & 0.58 & 8.43 & 0.53 \\
\hline 7 & 1.50 & 0.040 & 11.53 & 0.46 & 8.43 & 0.43 \\
\hline 8 & 1.75 & 0.030 & 15.69 & 0.47 & 10.17 & 0.45 \\
\hline 9 & 2.00 & 0.020 & 20.50 & 0.41 & 11.87 & 0.41 \\
\hline 10 & 3.00 & 0.010 & 46.12 & 0.46 & 18.49 & 0.53 \\
\hline 11 & 4.00 & 0.0063 & 82.00 & 0.52 & 24.97 & 0.67 \\
\hline 12 & 5.00 & 0.0023 & 128.12 & 0.29 & 31.40 & 0.43 \\
\hline 13 & 6.00 & 0.0010 & 184.49 & 0.18 & 37.80 & 0.29 \\
\hline 14 & 7.00 & 0.0006 & 251.11 & 0.15 & 44.18 & 0.26 \\
\hline 15 & 2.50 & 0.0088 & 32.03 & 0.28 & 15.21 & 0.30 \\
\hline
\end{tabular}


97. It was also desirable to generate waves that did not separate into multiple wave forms, or solitons, as discussed by Galvin (1970). Galvin defines solitons as multiple waves that separate from individual waves produced by wave generators in shallow water, and formation of these nonlinear waves is not restricted to any particular kind of equipment. Galvin suggests solitons develop because the wave generator motion does not match the water particle motion at the wave board. The wave conditions listed in Table 5 were plotted on Figure 25 (Galvin 1970) to predict the wave form that would be

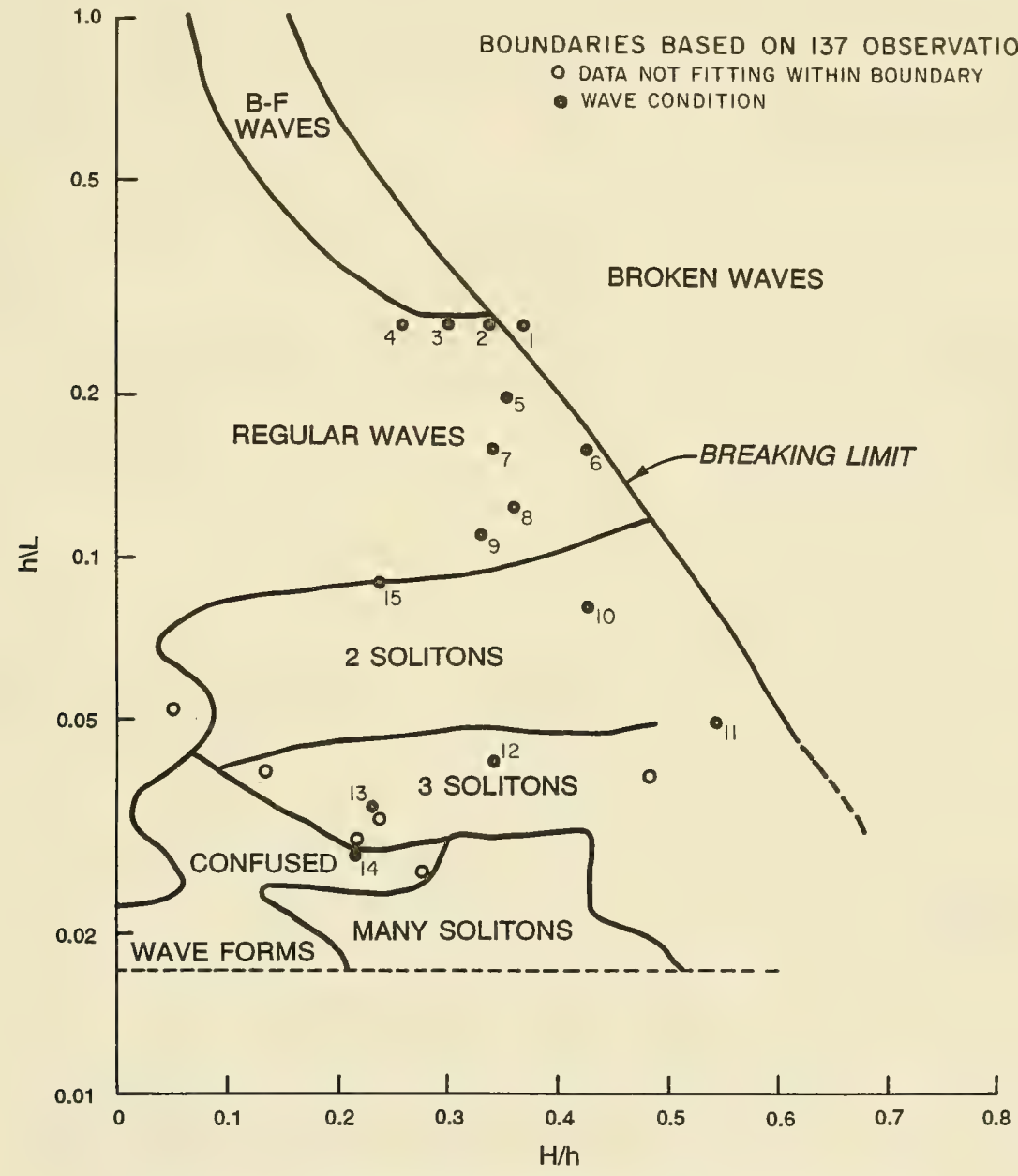

Figure 25. Predicted wave forms produced by a periodic wave generator, after Galvin (1970) 
generated in the tank. Wave Condition 1 falls on the broken waves-regular wave border. Wave Conditions 11-13 indicate two and three solitons may form. Condition 14 lies in the confused wave region, and Condition 15 falls on the border of regular waves and two solitons. Condition 15 was examined visually, and it was determined that no solitons formed. Regular waves were expected for Conditions 2-9. In an effort to generate only stable waves, and also to limit the total number of tests (about 100 tests), five stable wave conditions were selected with $\mathrm{H}_{\mathrm{o}} / \mathrm{L}_{\mathrm{o}}$-values ranging from 0.0088 to 0.09 . (The maximum wave steepness attainable is given by the Michell (1893) criterion $\mathrm{H}_{\mathrm{o}} / \mathrm{L}_{\circ}$ $=0.142)$. The low steepness wave, Condition 15, was included to cover a range in wave steepness of one order of magnitude. Condition 15 had the lowest wave steepness with the highest wave height that could be generated at $1.25-\mathrm{ft}$ SWL without producing solitons.

\section{Bar Design}

98. Selection of representative bar geometry was based on results of Larson and Kraus (1989). Larson and Kraus examined morphologic features produced in large wave tanks by regular waves (Saville 1957, Kajima et al. 1983, Kraus and Larson 1988). Saville conducted tests in a 635-ft-long, 15-ft-wide and 20-ft-deep wave tank, and Kajima et al. conducted tests in a 205-m-long, 3.4-m-wide, and 6-m-deep wave tank. Mean sand grain sizes were in the range of 0.20 to $0.47 \mathrm{~mm}$ in these experiments. Waves were of a field scale with heights reaching $1.8 \mathrm{~m}$, and periods were as long as $16 \mathrm{sec}$.

99. Larson and Kraus (1989) found that the equilibrium bars formed in these regular wave studies generally exhibited three predominant angles: a lower seaward angle $\beta_{1}$, an upper seaward angle $\beta_{2}$, and a shoreward angle $\beta_{3}$. The area covered by $\beta_{2}$ was fairly small and was a secondary angle. The average seaward angle $\beta_{1}$ of the bars ranged between 8 to 12 deg, with local slopes as great as $20 \mathrm{deg}$. The average shoreward bar angle for beach profiles near equilibrium was $28 \mathrm{deg}$, and ranged from 20 to $35 \mathrm{deg}$. Average secondary seaward bar angle $\beta_{2}$ ranged between 4 to $8 \mathrm{deg}$.

100. Bar geometry generated in a small tank was also examined in the present study. Fowler and Smith (1986) conducted movable-bed tests in the same tank used in this study. The purpose was to compare scaling relationships developed by Noda (1972), Lepetit and Leroy (1977), Vellinga (1982), 
Hughes (1983), and Hallermeiert for movable beds by modeling large-scale tests of Saville (1957). Sand sizes modeled in the laboratory cannot usually be scaled the same as distances and lengths because grain sizes with mean diameters less than $0.08 \mathrm{~mm}$ become cohesive and behave as clay and silt. The scaling relationships give distorted scales; that is, the horizontal and vertical scales are not the same, to compensate for larger sand sizes. The distorted scales gave initial slopes as steep as $1: 3.9$. Sand, coal, and glass beads were used as model sediments in the Fowler and Smith study, but only tests conducted with sand that resulted in erosional bar formations were analyzed to determine bar angles. The snall-scale cases analyzed in the present study consisted of sand sizes of 0.22 and $0.40 \mathrm{~mm}$, and wave heights ranging from 5.0 to $9.5 \mathrm{~cm}$.

101. The three predominant angles defined by Larson and Kraus (1989) were also found to be present in the smaller tank tests. The average angle for each group was calculated in the same manner as Larson and Kraus to determine $\beta_{1}, \beta_{2}$, and $\beta_{3}$. Average $\beta_{1}$ was $20 \mathrm{deg}$, ranging between 4 and $37 \mathrm{deg}$. Average $\beta_{3}$ was $8 \mathrm{deg}$ and ranged between 0 and $14 \mathrm{deg}$. A 0 -deg angle indicates a terrace bar system (Figure 26). Average $\beta_{2}$ was $6.5 \mathrm{deg}$. The steep seaward angles produced in the small-scale study were a result of the steep initial slopes.

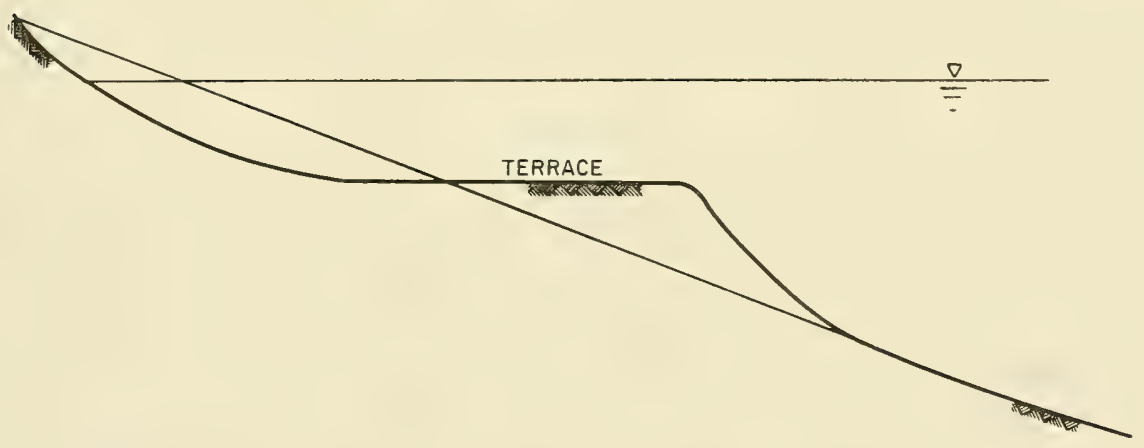

Figure 26. Sketch of terraced bar

* R. G. Hallermeier, 1984, "Unified Modeling Guidance Based on Sedimentation," Unpublished paper, US Army Engineer Waterways Experiment Station, Coastal. Engineering Research Center, Vicksburg, MS. 
102. Bars observed in the field typically have seaward angles less than $10 \mathrm{deg}$, as discussed by Larson and Kraus (1989). At least four reasons can be given to explain the gentleness of bar slope in the field:

a. Waves are irregular in height, period, and direction.

b. The water level varies with the tide.

c. Steady wave conditions do not exist.

d. Mechanisms other than short-period incident waves, such as undertow and infragravity waves, may contribute to move sand.

103. After examining bar angles from the aforementioned movable-bed studies performed with monochromatic waves, a range of bar angles was selected. The bars used in the experiment consisted of two angles, $\beta_{1}$ and $\beta_{3}$, which implies $\beta_{2}$ equals $\beta_{1}$. The secondary angle was not included since it covered only a small area of the bar, and it was felt that one seaward angle was sufficient for this initial study. The use of two bar angles resulted in bars that were either triangular (Figure 27) or, in the case of $\beta_{3}=0 \mathrm{deg}$, terraced. Seaward bar angles ranged from 5 to 40 deg, and shoreward bar angles ranged from 0 to $40 \mathrm{deg}$. The greater seaward angles are unrealistic for sand bars in the field; these were included because it was beneficial for engineering purposes to observe breaking waves on shapes that approximate those of submerged breakwaters or reefs, sills of perched beaches, and similar structures.

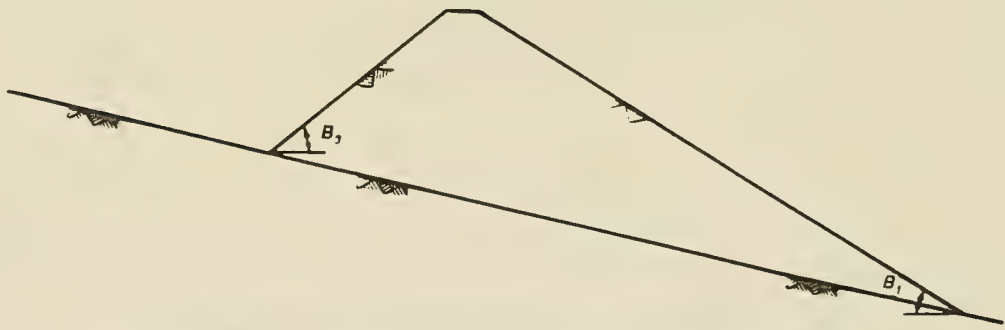

Figure 27. Shape of typical bar used in study

104. The location and size of the bars were required to produce breaking waves. Sunamura (1987) found a relationship between the depth of the bar 
crest $h_{c}$ and the breaking wave height. Larson and Kraus (1989) also observed that $h_{c}$ was related to breaking wave height and insensitive to wave period and beach and sand parameters. The depth at the bar crest could be determined from a simple equation:

$$
\mathrm{h}_{\mathrm{c}}=\mathrm{cH}_{\mathrm{b}}
$$

in which the value of the empirical coefficient $c$ was found to be 0.59 by Sunamura and 0.66 by Larson and Kraus. Equation 71 gives one point on the bar, and another point is required to specify the horizontal location and size of the bar.

105. Keulegan (1945) determined the ratio of $h_{t}$, the depth of the bar trough from SWL, to $h_{c}$ was 1.65 for bars in the field, and 1.69 for bars produced in the laboratory. Sallenger, Holman, and Birkemeier (1985) observed a bar during a storm and found $h_{t} / h_{c}=1.24$. Sunamura (1987) gave values of $h_{t} / h_{c}$ ranging from 1.16 to 1.93 based on field measurements on various coasts around the world. From results of large-wave tank tests (Saville 1957, Kajima et al. 1983), Larson and Kraus (1989) found an average value of $h_{t} / h_{c}=1.74$ and that $h_{t} / h_{c}$ was weakly dependent on wave steepness as

$$
\frac{h_{t}}{h_{c}}=2.5\left(\frac{H_{o}}{L_{o}}\right)^{0.092}
$$

106. Since the values of $h_{t} / h_{c}$ observed by Keulegan (1945), Sallenger, Holman, and Birkemeier (1985), Sunamura (1987), and Larson and Kraus (1989) differ, it was felt that use of a fixed value would be inappropriate. Equation 73 was selected to determine $h_{t} / h_{c}$, and values of this ratio ranged between 1.61 to 2.00 for the design wave conditions.

107. The depth of the bar crest was calculated using Equation 71, with $c=0.66$. The coefficient value of Larson and Kraus (1989) was selected, because it was obtained from the same data set used to determine Equation 72. Breaker height was estimated by Equation 30 (Sunamura 1981) for input into Equation 71. A program was written to calculate wave height through shoaling using linear wave theory at water depth increments of $0.1 \mathrm{ft}$. The wave height to water depth ratio was calculated at each depth increment and compared with 
$\gamma_{\mathrm{b}}$. The design breaking wave height was selected as the wave height at which $\mathrm{H} / \mathrm{h}$ equaled $\gamma_{\mathrm{b}}$.

108. Once estimates of $h_{t}$ and $h_{c}$ were determined by the abovedescribed procedure, the horizontal location of the bar was known, and the size of the bar could be determined. The depth at the bar trough served as a point of origin from which the shoreward face length was calculated from the given value of $\beta_{3}$ and the vertical distance between $h_{c}$ and $h_{t}$. After the shoreward bar face was calculated, the horizontal location of $h_{c}$ was known, and the seaward bar face length could be determined for the given seaward angle.

\section{$\underline{\text { Test Procedure }}$}

109. The bar was placed in the tank at the calculated crest depth. Since $h_{c}$ was based on an estimate of breaking wave height, waves were generated, and the actual breaking wave height was measured. The location of the bar was then adjusted to the correct $h_{c}$ if necessary. Adjustments to correct $h_{c}$ were typically less than $0.05 \mathrm{ft}$ vertically, and the maximum deviation from the calculated value was $0.07 \mathrm{ft}$.

110. Water surface elevations were measured with the gages sampling at $10 \mathrm{~Hz}$, from which statistical and spectrally defined wave parameters could be calculated. Wave breaking was recorded on videotape. Runup was also measured for each test by visually observing the maximum horizontal distance of the leading edge of the water past the shoreline for 12 or more successive waves, and then converting the distance to vertical elevation above SWL. Qualitative observations were made of the surf zone. Included in the experiment logbook were the depth of bubble penetration at the surf zone gages and the wave form (reformed wave or bore). If the broken wave reformed, the horizontal location of reformation was recorded. Additional notes consisted of the horizontal locations of the gages and water depths at the gages; horizontal location of the bar and water depth at the trough, crest, and toe of the bar; and the water temperature during the test.

111. Once a test was completed, the seaward face of the bar was replaced to give a new $B_{1}$. Wave height, wave period, and shoreward bar angle were held constant, and the newly constructed bar was placed in the tank for the next test. The preceding method was repeated until tests were made 
with all seaward bar angles for a given shoreward bar angle. A different shoreward bar face was attached to the bar, and waves were generated over the range of seaward angles. Wave height and period were changed to yield a different $\mathrm{H}_{0} / \mathrm{L}_{0}$ value after tests were made for all bar configurations, and the procedure was repeated.

\section{Pilot test}

112. A pilot test was performed as a trial of the methodology and validation of the criterion on bar depth given by Larson and Kraus (1989) prior to actual testing. In this pilot test, the wave conditions and equilibrium bar formed in a large wave tank test conducted by Saville (1957) was reproduced at the smaller scale of the present study.

113. Stive (1985) conducted tests in a large and small tank for similar wave steepnesses to make a scale comparison. Stive found that scale effects were not significant for wave height in the range of 0.15 to $1.5 \mathrm{~m}$. A large erosional bar was formed in Saville (1957) test CE400 (Kraus and Larson 1988); therefore, this case was selected to model. The deepwater wave steepness was scaled using the Froude model law (Stevens et al. 1942) in the small-scale experiment;

$$
\left(\frac{\mathrm{H}_{\mathrm{o}}}{\mathrm{L}_{\mathrm{o}}}\right)_{\mathrm{pr}}=\left(\frac{\mathrm{H}_{\mathrm{o}}}{\mathrm{L}_{\mathrm{o}}}\right)_{\mathrm{m}}
$$

in which the subscripts pr and $m$ denote prototype and model, respectively. Combining Equations 1 and 73 results in:

$$
\left(\mathrm{H}_{\mathrm{o}}\right)_{\mathrm{m}}=\left(\mathrm{H}_{\mathrm{o}}\right)_{\mathrm{pr}}\left(\frac{\mathrm{T}_{\mathrm{m}}}{\mathrm{T}_{\mathrm{pr}}}\right)^{2}
$$

A wave period was selected as input to Equation 74 that would result in a wave height which could be generated in the tank ( $h=1.25 \mathrm{ft}$ ) and which would also give a height greater than $0.15 \mathrm{~m}$. The model and prototype test results for Case CE400 are listed in Table 6. Although $H_{0} / L_{0}$ was slightly higher in the small-scale tests because of differences between the design and measured waves, the bar caused wave breaking and validated the criteria of Larson and 
Table 6

Prototype and Model Conditions of Case CE400 (Pilot Test)

\begin{tabular}{|c|c|c|c|c|c|c|c|}
\hline Study & $\mathrm{H}_{0} / \mathrm{L}_{0}$ & $\begin{array}{r}\mathrm{T} \\
\mathrm{sec} \\
\end{array}$ & $\begin{array}{l}L_{\circ} \\
\mathrm{ft}\end{array}$ & $\begin{array}{l}\mathrm{H}^{\prime} \\
\mathrm{ft}\end{array}$ & $\begin{array}{l}\mathrm{H}_{\mathrm{o}} \\
\mathrm{ft}\end{array}$ & $\begin{array}{c}\beta_{3} \\
\mathrm{deg} \\
\end{array}$ & $\begin{array}{c}\beta_{1} \\
\text { deg }\end{array}$ \\
\hline $\begin{array}{l}\text { Saville } \\
\text { (1957) }\end{array}$ & 0.035 & 5.6 & 160.6 & 5.32 & 5.63 & 11.8 & 8.4 \\
\hline $\begin{array}{l}\text { Present } \\
\text { Study }\end{array}$ & 0.040 & 1.46 & 10.9 & 0.41 & 0.44 & 11.8 & 8.4 \\
\hline
\end{tabular}

Kraus (1989); therefore, this method of determining bar size and location was used in all tests.

Monochromatic wave tests

114. Base tests consisted of various wave conditions and bar configurations for which three parameters were varied: $H_{0} / L_{0}, B_{1}$, and $B_{3}$. The tests involved five deepwater wave steepnesses, six seaward bar angles, and four shoreward bar angles. Design variables are listed in Table 7.

Table 7

List of Design* Parameters for Base Tests

\begin{tabular}{|c|c|c|c|c|}
\hline \multicolumn{3}{|c|}{ Parameter 1} & \multirow{2}{*}{ 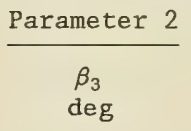 } & \multirow{2}{*}{ 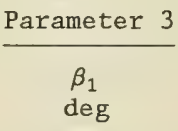 } \\
\hline $\mathrm{H}_{\mathrm{o}} / \mathrm{L}_{\mathrm{o}}$ & $\begin{array}{c}\mathrm{T} \\
\mathrm{sec}\end{array}$ & $\begin{array}{l}\mathrm{H}^{\prime} \\
\mathrm{ft}\end{array}$ & & \\
\hline 0.09 & 1.00 & 0.43 & 0 & 5 \\
\hline 0.07 & 1.00 & 0.33 & 20 & 10 \\
\hline 0.05 & 1.50 & 0.53 & 30 & 15 \\
\hline 0.03 & 1.75 & 0.45 & 40 & 20 \\
\hline \multirow[t]{2}{*}{0.0088} & 2.50 & 0.30 & & 30 \\
\hline & & & & 40 \\
\hline
\end{tabular}

* Nominal values for design purposes that were only approximated.

115. Six tests were also conducted for a design value of deepwater wave steepness $\left(H_{0} / L_{o}=0.05\right)$ for different combinations of wave height and period. Bar configurations consisting of three seaward bar angles (5, 10, and $15 \mathrm{deg}$ ) and one shoreward bar angle (20 deg) were subjected to two wave conditions 
$(1.25 \mathrm{sec}, 0.37 \mathrm{ft}$ and $1.00 \mathrm{sec}, 0.26 \mathrm{ft}$ ) in addition to the base tests with $\mathrm{H}_{\mathrm{o}} / \mathrm{L}_{\mathrm{o}}=0.05$. The purpose of this variation was to compare wave breaking properties over a range of heights and periods for a given value of $H_{0} / L_{\circ}$. These variations of the base tests are summarized in Table 8 .

$$
\text { Table } 8
$$

List of Design* Parameters for Base Test Variations

\begin{tabular}{|c|c|c|c|c|}
\hline \multicolumn{3}{|c|}{ Parameter 1} & \multirow{2}{*}{$\frac{\text { Parameter } 2}{\begin{array}{c}\beta_{3} \\
\operatorname{deg}\end{array}}$} & \multirow{2}{*}{ 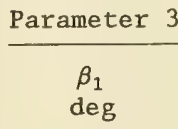 } \\
\hline $\mathrm{H}_{\mathrm{o}} / \mathrm{L}_{\mathrm{o}}$ & $\begin{array}{c}\mathrm{T} \\
\mathrm{sec}\end{array}$ & $\begin{array}{l}\mathrm{H}^{\prime} \\
\mathrm{ft}\end{array}$ & & \\
\hline \multirow[t]{3}{*}{0.05} & 1.25 & 0.37 & 20 & 5 \\
\hline & 1.00 & 0.26 & & 10 \\
\hline & & & & 15 \\
\hline
\end{tabular}

* Nominal values for design purposes which were only approximated.

116. Tests were made with base case waves without a bar for comparison of breaking wave properties with barred profiles. These tests provided a foundation for observing the influence of the bar on breaking wave properties. The plane slope tests were also included in analysis of previous data sets to obtain relationships for breaker indices and plunge distance.

117. Wave data were collected for $2 \mathrm{~min}$ for all monochromatic wave tests, but only 15 successive waves were analyzed. Analysis of the wave data was begun after waves reflected off the concrete slope and was ended before reflected waves from the wave board had returned to the bar. This procedure eliminated contamination of the data from waves reflected off the wave board and simulated the natural reflection of waves from the beach and bar. Breaking waves were also recorded on videotape for 2 min and quantities determined visually for 10 consecutive waves using the same procedure.

\section{Irregular wave tests}

118. Three irregular wave conditions were generated for three bar configurations each, as well as for the control case of no bar. A JONSWAP computer signal was generated for peak periods $\mathrm{T}_{\mathrm{p}}$ of $1.0,1.5$, and $1.75 \mathrm{sec}$ with significant wave heights $H_{s}$ of $0.37,0.47$, and $0.45 \mathrm{ft}$, respectively. Figure 28 shows the spectral shape generated for $\mathrm{T}_{\mathrm{p}}=1.75 \mathrm{sec}$, and the input parameters required to generate the signal; the wave height at the wave board, the water depth at the wave board, the spectral peak frequency 

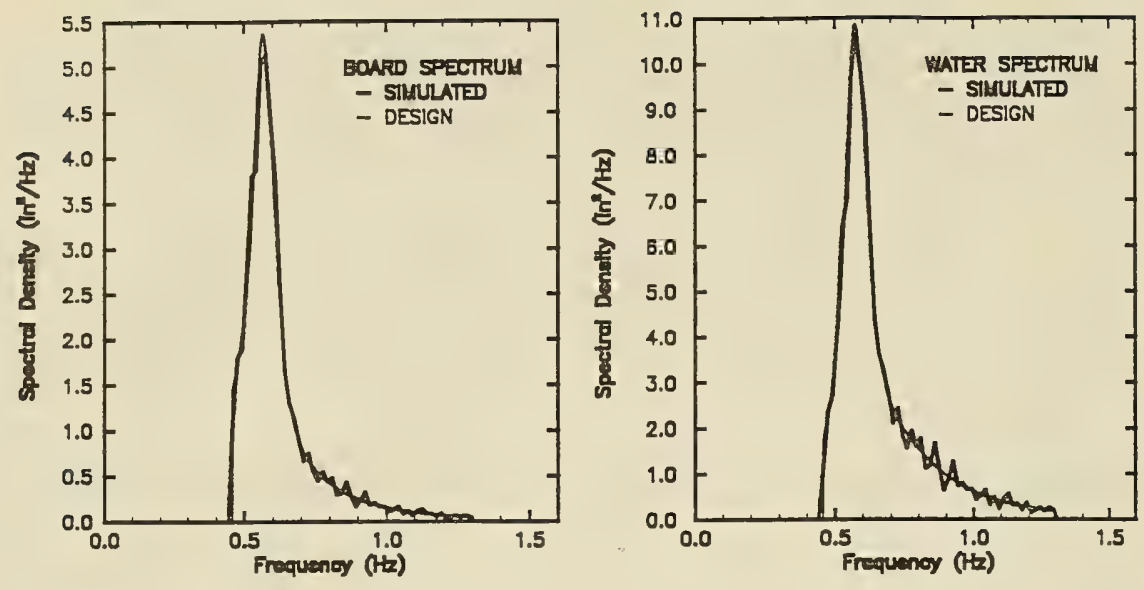

$$
\begin{aligned}
& \mathrm{H}^{\prime}=0.458 \mathrm{ft} \\
& \mathrm{f}_{\mathrm{p}}=0.571 \mathrm{~Hz} \\
& \sigma_{\mathrm{L}}=0.07
\end{aligned}
$$

$$
\begin{aligned}
\mathrm{h} & =1.25 \mathrm{ft} \\
\mathrm{G} & =3.3 \\
\sigma_{\mathrm{H}} & =0.09
\end{aligned}
$$

Figure 28. Spectral shape of irregular wave signal used to control wave board and predicted shape of water surface spectrum. ( $\sigma_{\mathrm{L}}$ and $\sigma_{\mathrm{H}}$ are low- and high-frequency spectral width parameters, respectively)

$f_{p}=1 / T_{p}$, the spectral width parameter $\sigma$, and the spectral peak enhancement parameter $\mathrm{G}$.

119. Peak deepwater wavelength $\left(\mathrm{L}_{\mathrm{p}}\right)_{\circ}$ and significant deepwater wave height $\left(H_{s}\right)$ 。 were calculated using linear wave theory with $T_{p}$ and $H_{s}$

\begin{tabular}{|c|c|c|c|c|}
\hline \multicolumn{3}{|c|}{ Parameter 1} & \multirow{2}{*}{ 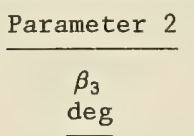 } & \multirow{2}{*}{ 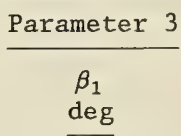 } \\
\hline$\left(H_{s} / L_{p}\right)_{\circ}$ & $\begin{array}{c}\mathrm{T}_{\mathrm{p}} \\
\text { sec }\end{array}$ & $\begin{array}{l}\mathrm{H}_{\mathrm{s}}^{\prime} \\
\mathrm{ft}\end{array}$ & & \\
\hline 0.078 & 1.00 & 0.37 & 20 & 5 \\
\hline 0.044 & 1.50 & 0.47 & & 10 \\
\hline 0.03 & 1.75 & 0.45 & & 15 \\
\hline
\end{tabular}
measured in the horizontal section of the tank as input. Wave data were collected and analyzed for 500 waves for the irregular wave tests. Table 9 lists design parameters for the irregular wave tests.

Table 9

Irregular Wave Test Design ${ }^{\star}$ Parameters

* Nominal values for design purposes which were only approximated.

** $\mathrm{H}_{\mathrm{S}}^{\prime}=$ significant wave height in horizontal section of task. 


\section{PART IV: RESULTS}

120. Principal results of the study are presented in this chapter. Breaker heights and depths, plunge and splash distances, and vortex areas were determined from videotape. Measurements were made for 10 successive waves and averaged for use in analysis. The video playback device was a sony 5650 professional editing machine, consisting of two playback machines and one 13-in. and one 19-in. monitor. Playback speed could be varied down to freeze frame. Wave height decay and wave reflection were obtained by analysis of resistancetype wave gage records. The horizontal uprush of the wave was determined visually and converted to a vertical distance above SWL to acquire wave runup. Breaker type was also observed and recorded during testing.

121. Totally, 120 tests were performed, including 96 base tests with monochromatic waves, 11 variations of the base monochromatic wave tests, and 12 tests with irregular waves. The first monochromatic test modeled the wave conditions over a solid model of the bar formed during a movable-bed test conducted by Saville (1957) in a large wave tank. This pilot test was performed to validate the bar depth criterion of Larson and Kraus (1989) to be used to design the bars.

122. Visual observations during the series of tests showed that the return flow over the shoreward slope of the bars influenced the breaking wave characteristics. A strong return flow was present if the cross-sectional area of the surf zone was small, such as for tests with terraced bars. The return flow also appeared to promote formation of a secondary wave in the trough of the incident wave if $\beta_{1}$ was large compared with $\mathrm{H}_{0} / \mathrm{L}_{0}$. As water flowed seaward over the bar, the water surface profile conformed to the shape of the bar, much like critical water flow over a weir. For steeper bars, the water surface profile over the bar was also steep, and the incident wave tended to collapse or "trip" over the bar, rather than shoal and break by the depthlimiting mechanism. The seaward bar angle necessary to cause wave tripping decreased as $H_{0} / L_{0}$ decreased; therefore, collapsing waves occurred for gentler bar slopes with smaller wave steepnesses. Performance of tests over the entire range of seaward bar angles with the smaller deepwater wave steepnesses was unnecessary because of the abnormal wave tripping effect.

123. The influence of shoreward bar angle on wave breaking was not found, which was attributable to the return flow. To quantify the effect of 
shoreward bar angle on wave breaking, return flow measurements will be necessary in future studies. The data points shown in the figures of this chapter represent results from individual tests with different $\beta_{3}$-angles, unless otherwise noted.

124. A list of the base tests, including the control cases with no bar, is given in Table 10. The first three digits of the case number specify the deepwater wave steepness, shoreward bar angle, and seaward bar angle, respectively. A fourth digit was used to differentiate tests that had identical wave and bar conditions. Results of the data analysis for the monochromatic wave tests can be found in Tables A1 and A2. Table 11 summarizes the irregular wave tests. The letter " $R$ " preceding the four-digit case number in Table 11 indicates that irregular, or random, waves were generated.

\section{Breaker Type}

125. Three breaker types were observed during the tests: spilling, plunging, and collapsing. Breaker type transition values observed in the experiment are shown as a function of offshore surf similarity parameter in the upper portion of Figure 29. The offshore surf similarity parameter was calculated by substituting $\tan \beta_{1}$ for the slope $m$ in Equation 4 (Battjes 1975) and using linear wave theory to calculate $H_{0}$ and $L_{0}$ from wave heights and periods measured at the gage in the horizontal section of the tank. Transition values between breaker types given by Battjes for plane slopes are shown in the lower portion of Figure 29. Both plunging and collapsing breakers in the present study for barred profiles occurred for lower $\xi_{0}$-values than predicted by the plane-slope values. Transition values for barred profiles in this study were:

$$
\begin{array}{rr}
\text { surging or collapsing if } & \xi_{0}>1.2 \\
\text { plunging if } & 0.4<\xi_{0}<1.2 \\
\text { spilling if } & \xi_{0}<0.4
\end{array}
$$

The lower transition values show that some waves that would break by spilling on a plane slope will plunge if a bar is present, and some waves that would plunge on a plane slope collapse on a barred profile. 
Table 10

Summary of Monochromatic Base Tests

\begin{tabular}{|c|c|c|c|c|c|c|c|c|}
\hline Case & $\begin{array}{c}\mathrm{T} \\
\mathrm{sec} \\
\end{array}$ & $\mathrm{H}_{\mathrm{o}} / \mathrm{L}_{\mathrm{o}}$ & $\begin{array}{l}L_{o} \\
\mathrm{ft}\end{array}$ & $\begin{array}{l}\mathrm{H}_{\circ} \\
\mathrm{ft}\end{array}$ & $\begin{array}{l}L^{\prime} \\
\text { ft }\end{array}$ & $\begin{array}{l}\mathrm{H}^{\prime} \\
\mathrm{ft}\end{array}$ & $\begin{array}{c}\beta_{1} \\
\operatorname{deg}\end{array}$ & $\begin{array}{l}\beta_{3}{ }^{*} \\
\operatorname{deg}\end{array}$ \\
\hline $2000^{\star \star}$ & 1.02 & 0.092 & 5.29 & 0.49 & 4.88 & 0.45 & -- & -- \\
\hline 2110 & 1.02 & 0.095 & 5.33 & 0.50 & 4.91 & 0.47 & 5.0 & 0 \\
\hline 2120 & 1.02 & 0.095 & 5.33 & 0.51 & 4.91 & 0.47 & 9.5 & 0 \\
\hline 2130 & 1.02 & 0.094 & 5.32 & 0.50 & 4.91 & 0.47 & 13.8 & 0 \\
\hline 2140 & 1.01 & 0.091 & 5.27 & 0.48 & 4.87 & 0.45 & 19.5 & 0 \\
\hline 2150 & 1.02 & 0.096 & 5.29 & 0.51 & 4.88 & 0.47 & $30^{*}$ & 0 \\
\hline 2160 & 1.02 & 0.097 & 5.31 & 0.52 & 4.90 & 0.48 & $40^{*}$ & 0 \\
\hline 2211 & 1.02 & 0.089 & 5.33 & 0.47 & 4.91 & 0.44 & 5.8 & 20 \\
\hline 2212 & 1.02 & 0.089 & 5.33 & 0.48 & 4.91 & 0.44 & $5^{\pi}$ & 20 \\
\hline 2220 & 1.02 & 0.091 & 5.33 & 0.49 & 4.91 & 0.45 & 11.8 & 20 \\
\hline 2230 & 1.02 & 0.092 & 5.31 & 0.49 & 4.90 & 0.45 & 12.0 & 20 \\
\hline 2240 & 1.02 & 0.093 & 5.31 & 0.49 & 4.90 & 0.46 & 19.0 & 20 \\
\hline 2250 & 1.02 & 0.088 & 5.30 & 0.47 & 4.89 & 0.43 & $30^{*}$ & 20 \\
\hline 2260 & 1.02 & 0.088 & 5.31 & 0.47 & 4.90 & 0.44 & $40^{*}$ & 20 \\
\hline 2310 & 1.02 & 0.091 & 5.32 & 0.48 & 4.91 & 0.45 & 5.3 & 30 \\
\hline 2320 & 1.02 & 0.088 & 5.32 & 0.47 & 4.91 & 0.43 & 9.5 & 30 \\
\hline 2330 & 1.02 & 0.093 & 5.31 & 0.49 & 4.90 & 0.46 & 14.7 & 30 \\
\hline 2340 & 1.02 & 0.093 & 5.31 & 0.49 & 4.90 & 0.46 & 20.6 & 30 \\
\hline 2350 & 1.02 & 0.091 & 5.31 & 0.48 & 4.90 & 0.45 & $30^{*}$ & 30 \\
\hline 2360 & 1.02 & 0.090 & 5.31 & 0.48 & 4.90 & 0.44 & $40^{*}$ & 30 \\
\hline 2410 & 1.02 & 0.091 & 5.33 & 0.48 & 4.91 & 0.45 & 5.3 & 40 \\
\hline 2420 & 1.02 & 0.090 & 5.30 & 0.47 & 4.89 & 0.44 & 9.8 & 40 \\
\hline 2430 & 1.02 & 0.088 & 5.32 & 0.47 & 4.91 & 0.44 & 14.8 & 40 \\
\hline 2440 & 1.02 & 0.091 & 5.31 & 0.48 & 4.90 & 0.45 & 19.0 & 40 \\
\hline 2450 & 1.02 & 0.092 & 5.31 & 0.49 & 4.90 & 0.45 & $30^{*}$ & 40 \\
\hline 2460 & 1.02 & 0.093 & 5.32 & 0.50 & 4.91 & 0.46 & $40^{*}$ & 40 \\
\hline $4000^{* \hbar}$ & 1.02 & 0.066 & 5.31 & 0.35 & 4.90 & 0.32 & $\cdots$ & -- \\
\hline 4110 & 1.02 & 0.069 & 5.28 & 0.36 & 4.87 & 0.34 & 4.8 & 0 \\
\hline 4120 & 1.02 & 0.070 & 5.28 & 0.37 & 4.87 & 0.34 & 9.8 & 0 \\
\hline 4130 & 1.02 & 0.070 & 5.28 & 0.37 & 4.87 & 0.34 & 14.1 & 0 \\
\hline 4140 & 1.01 & 0.070 & 5.26 & 0.37 & 4.86 & 0.34 & 19.0 & 0 \\
\hline 4150 & 1.02 & 0.072 & 5.28 & 0.38 & 4.87 & 0.35 & $30^{\star}$ & 0 \\
\hline 4160 & 1.02 & 0.073 & 5.29 & 0.39 & 4.88 & 0.36 & $40^{*}$ & 0 \\
\hline 4211 & 1.02 & 0.082 & 5.30 & 0.43 & 4.89 & 0.40 & 5.2 & 20 \\
\hline 4212 & 1.02 & 0.069 & 5.30 & 0.37 & 4.89 & 0.34 & $5^{*}$ & 20 \\
\hline 4220 & 1.02 & 0.075 & 5.30 & 0.40 & 4.89 & 0.37 & 11.4 & 20 \\
\hline 4230 & 1.02 & 0.075 & 5.30 & 0.40 & 4.89 & 0.37 & 14.3 & 20 \\
\hline 4240 & 1.02 & 0.076 & 5.30 & 0.40 & 4.89 & 0.38 & 19.0 & 20 \\
\hline 4250 & 1.02 & 0.077 & 5.30 & 0.41 & 4.89 & 0.38 & $30^{*}$ & 20 \\
\hline 4260 & 1.02 & 0.070 & 5.28 & $\begin{array}{c}0.37 \\
\text { ntinu }\end{array}$ & 4.87 & 0.34 & $40^{*}$ & 20 \\
\hline
\end{tabular}

Nominal values used.

** No bar. 
Table 10 (Continued)

\begin{tabular}{|c|c|c|c|c|c|c|c|c|}
\hline Case & $\begin{array}{c}\mathrm{T} \\
\mathrm{sec}\end{array}$ & $\mathrm{H}_{\mathrm{o}} / \mathrm{L}_{\mathrm{o}}$ & $\begin{array}{l}\mathrm{L}_{\mathrm{o}} \\
\mathrm{ft}\end{array}$ & $\begin{array}{l}\mathrm{H}_{\mathrm{o}} \\
\mathrm{ft}\end{array}$ & $\begin{array}{l}L^{\prime} \\
\mathrm{ft}\end{array}$ & $\begin{array}{l}\mathrm{H}^{\prime} \\
\mathrm{ft}\end{array}$ & $\begin{array}{c}\beta_{1} \\
\text { deg }\end{array}$ & $\begin{array}{l}\beta_{3}{ }^{*} \\
\text { deg }\end{array}$ \\
\hline 4310 & 1.02 & 0.069 & 5.29 & 0.37 & 4.88 & 0.34 & 4.6 & 30 \\
\hline 4320 & 1.02 & 0.070 & 5.29 & 0.37 & 4.88 & 0.34 & 10.4 & 30 \\
\hline 4330 & 1.02 & 0.069 & 5.29 & 0.36 & 4.88 & 0.34 & 13.2 & 30 \\
\hline 4340 & 1.02 & 0.068 & 5.29 & 0.36 & 4.88 & 0.34 & 17.4 & 30 \\
\hline 4350 & 1.02 & 0.069 & 5.30 & 0.36 & 4.89 & 0.34 & $30^{\star}$ & 30 \\
\hline 4360 & 1.02 & 0.068 & 5.29 & 0.36 & 4.88 & 0.33 & $40^{*}$ & 30 \\
\hline 4410 & 1.02 & 0.069 & 5.30 & 0.36 & 4.89 & 0.34 & 5.5 & 40 \\
\hline 4420 & 1.02 & 0.069 & 5.30 & 0.37 & 4.89 & 0.34 & 11.5 & 40 \\
\hline 4430 & 1.02 & 0.069 & 5.30 & 0.36 & 4.89 & 0.34 & 12.9 & 40 \\
\hline 4440 & 1.02 & 0.069 & 5.30 & 0.37 & 4.89 & 0.34 & 20.6 & 40 \\
\hline 4450 & 1.02 & 0.069 & 5.30 & 0.37 & 4.89 & 0.34 & $30^{*}$ & 40 \\
\hline 4460 & 1.02 & 0.070 & 5.29 & 0.37 & 4.88 & 0.34 & $40^{*}$ & 40 \\
\hline $6000^{* \hbar t}$ & 1.49 & 0.046 & 11.35 & 0.52 & 8.35 & 0.48 & - - & -- \\
\hline 6111 & 1.49 & 0.046 & 11.39 & 0.52 & 8.37 & 0.48 & $5^{*}$ & 0 \\
\hline 6112 & 1.50 & 0.047 & 11.45 & 0.54 & 8.40 & 0.50 & 5.4 & 0 \\
\hline 6120 & 1.49 & 0.048 & 11.36 & 0.55 & 8.35 & 0.51 & 9.7 & 0 \\
\hline 6130 & 1.50 & 0.052 & 11.50 & 0.60 & 8.42 & 0.56 & 13.6 & 0 \\
\hline 6140 & 1.50 & 0.048 & 11.50 & 0.55 & 8.42 & 0.51 & 21.6 & 0 \\
\hline 6210 & 1.49 & 0.048 & 11.33 & 0.55 & 8.34 & 0.51 & 5.2 & 20 \\
\hline 6220 & 1.49 & 0.043 & 11.44 & 0.50 & 8.39 & 0.46 & 9.7 & 20 \\
\hline 6230 & 1.49 & 0.042 & 11.44 & 0.48 & 8.39 & 0.44 & 14.2 & 20 \\
\hline 6240 & 1.50 & 0.043 & 11.55 & 0.50 & 8.44 & 0.46 & 20.6 & 20 \\
\hline 6250 & 1.50 & 0.041 & 11.58 & 0.47 & 8.45 & 0.44 & $30^{*}$ & 20 \\
\hline 6310 & 1.47 & 0.048 & 11.10 & 0.53 & 8.23 & 0.49 & 4.9 & 30 \\
\hline 6320 & 1.49 & 0.043 & 11.44 & 0.50 & 8.39 & 0.46 & 10.6 & 30 \\
\hline 6330 & 1.50 & 0.043 & 11.53 & 0.49 & 8.43 & 0.45 & 15.7 & 30 \\
\hline 6340 & 1.50 & 0.042 & 11.50 & 0.48 & 8.42 & 0.45 & 19.0 & 30 \\
\hline 6410 & 1.49 & 0.046 & 11.39 & 0.53 & 8.37 & 0.49 & 5.3 & 40 \\
\hline 6420 & 1.49 & 0.043 & 11.44 & 0.50 & 8.39 & 0.46 & 10.4 & 40 \\
\hline 6430 & 1.50 & 0.043 & 11.50 & 0.50 & 8.42 & 0.46 & 14.0 & 40 \\
\hline 6440 & 1.50 & 0.043 & 11.48 & 0.50 & 8.41 & 0.46 & 20.6 & 40 \\
\hline $8000^{* \pi}$ & 1.74 & 0.030 & 15.43 & 0.47 & 10.07 & 0.45 & -- & -- \\
\hline 8110 & 1.74 & 0.031 & 15.52 & 0.48 & 10.10 & 0.46 & 5.6 & 0 \\
\hline 8120 & 1.74 & 0.031 & 15.53 & 0.49 & 10.11 & 0.47 & 10.1 & 0 \\
\hline 8130 & 1.74 & 0.032 & 15.57 & 0.50 & 10.12 & 0.48 & 14.0 & 0 \\
\hline 8140 & 1.74 & 0.032 & 15.59 & 0.50 & 10.13 & 0.48 & 19.0 & 0 \\
\hline 8210 & 1.74 & 0.032 & 15.53 & 0.50 & 10.11 & 0.48 & 5.3 & 20 \\
\hline 8220 & 1.74 & 0.034 & 15.55 & 0.52 & 10.12 & 0.50 & 10.5 & 20 \\
\hline 8230 & 1.74 & 0.034 & 15.55 & 0.52 & 10.12 & 0.50 & 14.0 & 20 \\
\hline 8240 & 1.74 & 0.034 & 15.53 & 0.53 & 10.11 & 0.51 & 23.6 & 20 \\
\hline \multicolumn{9}{|c|}{ (Continued) } \\
\hline
\end{tabular}

* Nominal values used.

** No bar. 
Table 10 (Concluded)

\begin{tabular}{|c|c|c|c|c|c|c|c|c|}
\hline Case & $\begin{array}{c}\mathrm{T} \\
\mathrm{sec}\end{array}$ & $\mathrm{H}_{\mathrm{o}} / \mathrm{L}_{\mathrm{o}}$ & $\begin{array}{l}\mathrm{L}_{\mathrm{o}} \\
\mathrm{ft}\end{array}$ & $\begin{array}{l}\mathrm{H}_{\mathrm{o}} \\
\mathrm{ft}\end{array}$ & $\begin{array}{l}L^{\prime} \\
\mathrm{ft}\end{array}$ & $\begin{array}{l}\mathrm{H}^{\prime} \\
\mathrm{ft}\end{array}$ & $\begin{array}{c}\beta_{1} \\
\mathrm{deg} \\
\end{array}$ & $\begin{array}{l}\beta_{3}{ }^{\star} \\
\mathrm{deg}\end{array}$ \\
\hline 8310 & 1.74 & 0.032 & 15.52 & 0.50 & 10.10 & 0.48 & 5.0 & 30 \\
\hline 8320 & 1.74 & 0.034 & 15.52 & 0.52 & 10.10 & 0.50 & 11.0 & 30 \\
\hline 8330 & 1.74 & 0.034 & 15.52 & 0.53 & 10.10 & 0.50 & 16.5 & 30 \\
\hline 8340 & 1.74 & 0.034 & 15.52 & 0.53 & 10.10 & 0.50 & 22.1 & 30 \\
\hline 8410 & 1.74 & 0.033 & 15.53 & 0.51 & 10.11 & 0.48 & 5.6 & 40 \\
\hline 8420 & 1.74 & 0.034 & 15.52 & 0.52 & 10.10 & 0.50 & 11.2 & 40 \\
\hline 8430 & 1.74 & 0.034 & 15.53 & 0.52 & 10.11 & 0.50 & 16.5 & 40 \\
\hline 8440 & 1.74 & 0.034 & 15.50 & 0.53 & 10.10 & 0.50 & 20.6 & 40 \\
\hline $10000^{* \hbar}$ & 2.49 & 0.009 & 31.65 & 0.28 & 15.11 & 0.30 & -. & -- \\
\hline 10110 & 2.49 & 0.010 & 31.65 & 0.32 & 15.11 & 0.34 & 5.4 & 0 \\
\hline 10120 & 2.48 & 0.008 & 31.62 & 0.26 & 15.10 & 0.27 & 9.2 & 0 \\
\hline 10130 & 2.49 & 0.008 & 31.72 & 0.25 & 15.13 & 0.27 & 12.9 & 0 \\
\hline 10210 & 2.49 & 0.009 & 31.65 & 0.28 & 15.11 & 0.30 & 5.6 & 20 \\
\hline 10220 & 2.49 & 0.009 & 31.67 & 0.28 & 15.12 & 0.30 & 11.9 & 20 \\
\hline 10230 & 2.49 & 0.008 & 31.65 & 0.26 & 15.11 & 0.27 & 17.4 & 20 \\
\hline 10310 & 2.49 & 0.008 & 31.72 & 0.26 & 15.13 & 0.28 & 5.0 & 30 \\
\hline 10320 & 2.49 & 0.008 & 31.75 & 0.26 & 15.14 & 0.28 & 12.3 & 30 \\
\hline 10330 & 2.49 & 0.008 & 31.72 & 0.25 & 15.13 & 0.27 & 15.7 & 30 \\
\hline 10410 & 2.49 & 0.008 & 31.75 & 0.25 & 15.14 & 0.27 & 5.2 & 40 \\
\hline 10420 & 2.49 & 0.008 & 31.72 & 0.25 & 15.13 & 0.26 & 9.8 & 40 \\
\hline 10430 & 2.49 & 0.008 & 31.70 & 0.25 & 15.12 & 0.27 & 15.7 & 40 \\
\hline
\end{tabular}

* Nominal values used.

** No bar.

(Sheet 3 of 3 ) 
Table 11

Summary of Irregular Wave Tests

\begin{tabular}{|c|c|c|c|c|c|c|c|}
\hline Case & $\begin{array}{c}\beta_{1}^{*} \\
\operatorname{deg}\end{array}$ & $\begin{array}{c}T_{p} \\
\text { sec } \\
\end{array}$ & $\begin{array}{c}\left(L_{p}\right)_{0} \\
f t\end{array}$ & $\begin{array}{c}\left(\mathrm{H}_{\mathrm{rms}}\right)_{0} \\
\mathrm{ft}\end{array}$ & $\begin{array}{c}\left(\mathrm{H}_{\mathrm{s}}\right)_{0} \\
\mathrm{ft} \\
\end{array}$ & $\left(\mathrm{H}_{\mathrm{rms}}\right)_{0} /\left(\mathrm{L}_{\mathrm{p}}\right)_{0}$ & $\left(\mathrm{H}_{\mathrm{s}}\right)_{0} /\left(\mathrm{L}_{\mathrm{p}}\right)_{0}$ \\
\hline $\mathrm{R} 2000^{* *}$ & - & 1.07 & 5.88 & 0.30 & 0.41 & 0.051 & 0.064 \\
\hline R2210 & 5 & 1.07 & 5.88 & 0.29 & 0.40 & 0.050 & 0.063 \\
\hline R2220 & 10 & 1.07 & 5.81 & 0.30 & 0.41 & 0.051 & 0.064 \\
\hline R2230 & 15 & 1.07 & 5.81 & 0.30 & 0.41 & 0.051 & 0.065 \\
\hline $\mathrm{R} 6000^{* *}$ & - & 1.52 & 11.78 & 0.38 & 0.51 & 0.032 & 0.040 \\
\hline R6210 & 5 & 1.56 & 12.42 & 0.38 & 0.50 & 0.030 & 0.037 \\
\hline $\mathrm{R} 6220$ & 10 & 1.56 & 12.39 & 0.38 & 0.50 & 0.031 & 0.038 \\
\hline R6230 & 15 & 1.57 & 12.68 & 0.38 & 0.51 & 0.030 & 0.038 \\
\hline $\mathrm{R} 8000^{\text {** }}$ & - & 1.74 & 15.50 & 0.35 & 0.47 & 0.022 & 0.029 \\
\hline R8210 & 5 & 1.75 & 15.61 & 0.34 & 0.47 & 0.022 & 0.029 \\
\hline R8220 & 10 & 1.74 & 15.50 & 0.34 & 0.47 & 0.022 & 0.029 \\
\hline $\mathrm{R} 8230$ & 15 & 1.75 & 15.64 & 0.34 & 0.47 & 0.022 & 0.028 \\
\hline
\end{tabular}

* Nominal values used.

** No bar.

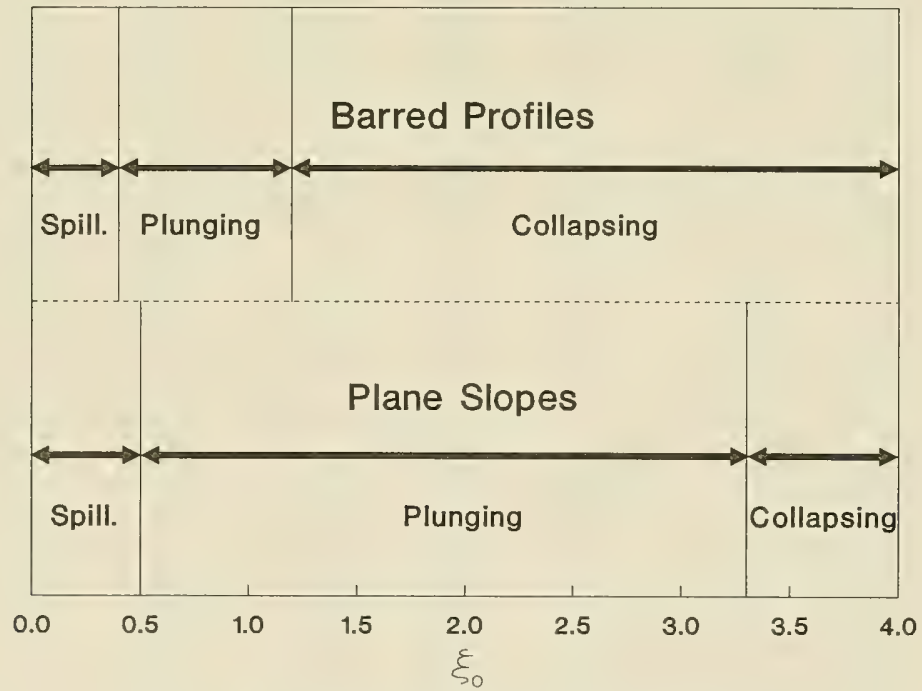

Figure 29. Breaker type as a function of $\xi_{0}$ 
126. Breaker type was also subjectively classified as "well-behaved" or "confused." The well-behaved breakers were waves that broke by depth-limited conditions (spilling and plunging breakers), and the confused breakers were believed to be waves that broke as a result of strong return flow (collapsing breakers). A relative length parameter was introduced to quantify wellbehaved and confused breakers. The relative length parameter was defined as the ratio of the length of the seaward bar face $s_{1}$, i.e., the horizontal distance from the bar crest to the seaward toe of the bar, to the wavelength at breaking $\mathrm{I}_{\mathrm{b}}$. The wavelength at breaking was calculated by linear shallow-water wave theory as

$$
\mathrm{L}_{\mathrm{b}}=\mathrm{T}\left(g \mathrm{~h}_{\mathrm{b}}\right)^{1 / 2}
$$

Figure 30 shows well-behaved and confused breakers as a function of the relative length parameter. Well-behaved breaking occurred for $s_{1} / L_{b} \geq 0.75$, which means well-behaved or depth-limited breaking occurs if $s_{1}$ is at least 75 percent as long as the wavelength at breaking. If $s_{1}<0.75 \mathrm{~L}_{\mathrm{b}}$, the bar probably had little effect on wave deformation leading up to breaking and, instead of gradually shoaling, waves broke by tripping over the bar.

127. Under natural conditions on a sandy beach, the bar and incident

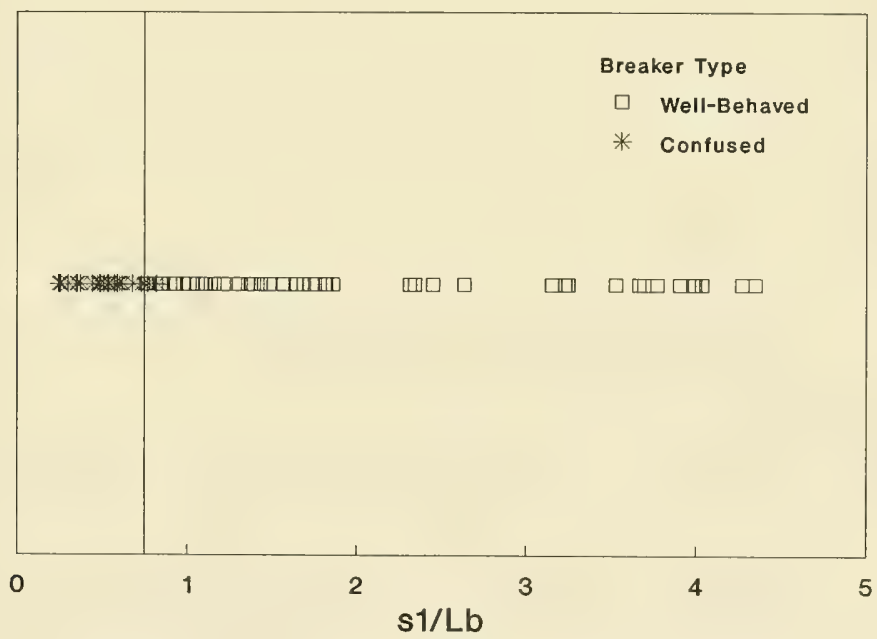

Figure 30. Breaker type as a function of the relative length parameter 
waves interact to provide an equilibrium profile. Bars are gentler under these conditions than those that cause confused breaking. Confused breaking may occur for engineered conditions, such as in areas where dredged material is disposed. However, if waves possess sufficient energy, they will initiate sediment movement, material will be transported, and the interaction between the bar and waves will form a more gently sloping bar shape.

\section{Wave Reflection}

128. Reflection coefficients $K_{r}$ obtained from wave heights at Gages 2 and 3 and the predicted values of Equation 8 (Miche 1951) and Equation 10 (Battjes 1975) were plotted as a function of $\xi_{0}$, shown in Figure 31 . The data are scattered, and one data point is anomalously high $\left(K_{r}=0.48\right)$. The reason this point is high is not known, and it was disregarded in all analysis of reflection. Although there is scatter, a clear trend is present for $K_{r}$ to be constant $(0.15)$ for all values of $\xi_{0}$. Predictions of the Miche equation result in a steep curve that approaches a perfectly reflected wave for bar and wave conditions if $\xi_{0} \approx 1$. Equation 10 underpredicts $\mathrm{K}_{\mathrm{r}}$ at low

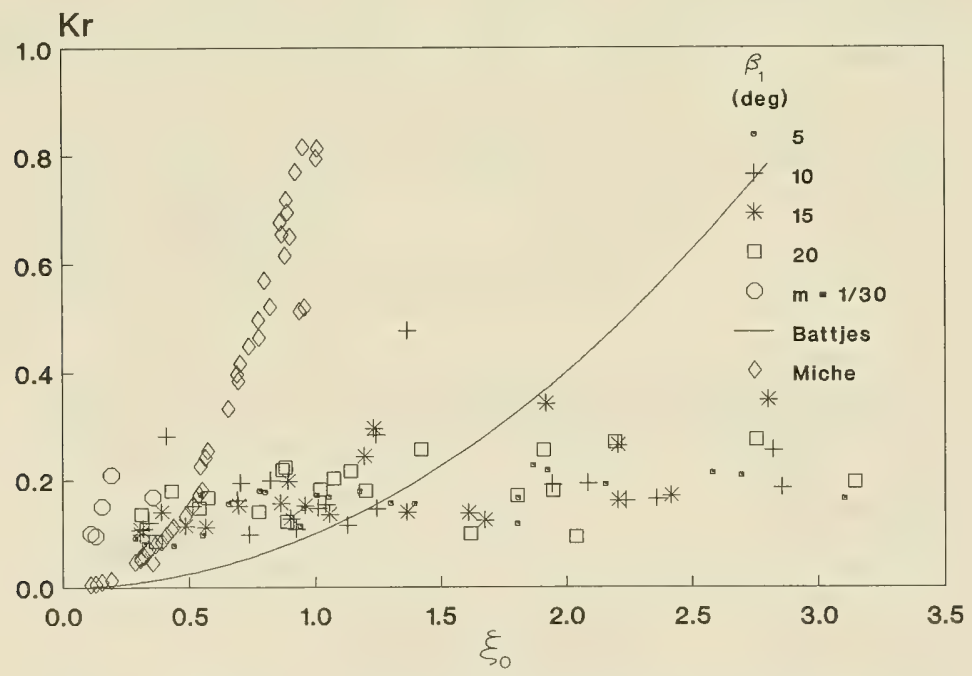

Figure 31. Comparison of measured $\mathrm{K}_{\mathrm{r}}$ with predicted values of Battjes (1975) and Miche (1951) as a function of $\xi_{0}$ 
values of $\xi_{0}$ (including the plane slope data) and overpredicts $K_{r}$ at higher $\xi_{0}$-values. The Battjes equation predicts increasing reflection as the surf similarity parameter increases, whereas the data show little dependence on $\xi_{0}$. Neither the equation of Miche nor that of Battjes predicts $K_{r}$ well for barred profiles over the range of $\xi_{0}$-values. These equations were developed for plane slopes and, as Figure 31 illustrates, are not valid if the bottom topography is irregular.

129. Reflection coefficients from the experiment were compared with Equation 11 (Ahrens 1987), shown in Figure 32, but no strong correlation was found with the reef reflection parameter $\mathrm{P}$. Equation 11 predicts $\mathrm{K}_{\mathrm{r}}$ better than Equations 8 (Miche 1951) and 10 (Battjes 1975), but does not exhibit a constant trend. The Ahrens equation does have the desirable property of remaining bounded, as opposed to Equations 8 and 10 .

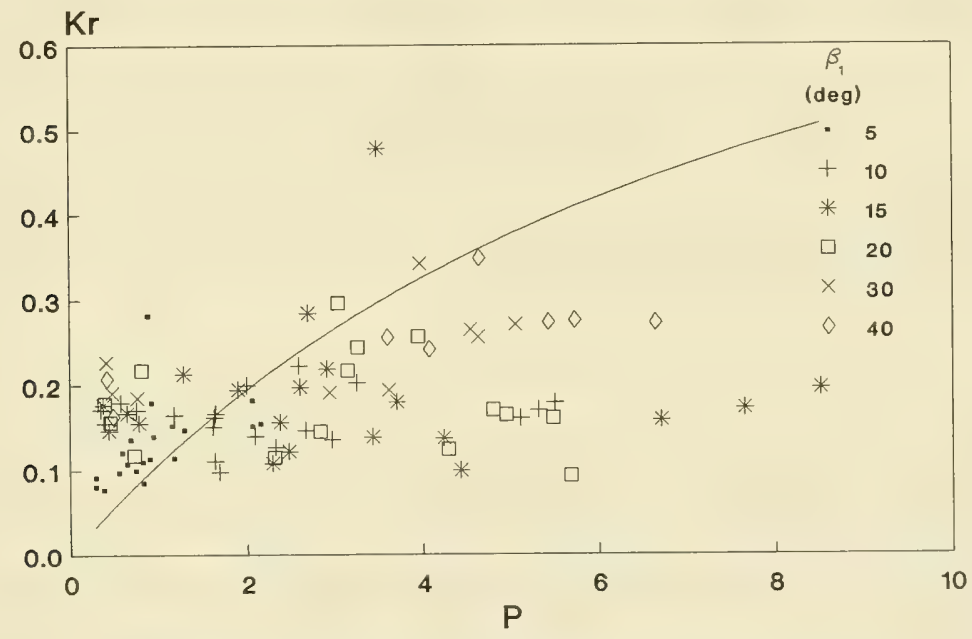

Figure 32. Measured wave reflection and predicted values of Ahrens (1987) as a function of $P$

130. An empirical equation was developed to predict $K_{r}$. Reflection coefficients from tests with and without bars were plotted as a function of $\beta_{1}$ (Figure 33), which shows a weakly increasing dependence of $\mathrm{K}_{\mathrm{r}}$ on $\beta_{1}$. The visually fit curve represents:

$$
\mathrm{K}_{\mathrm{r}}=0.132+0.119 \tan \beta_{1}
$$




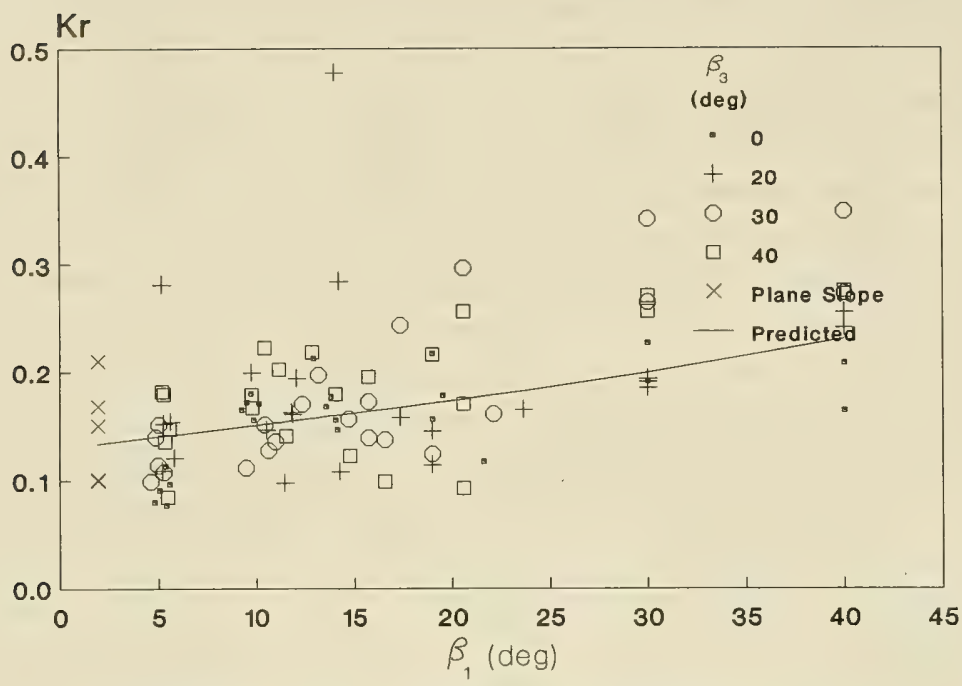

Figure 33. Wave reflection as a function of $\beta_{1}$

for $1.9 \mathrm{deg} \leq \beta_{1} \leq 40 \mathrm{deg}$. The results indicate that $K_{r}$ for barred profiles is effectively independent of wave steepness.

\section{Breaker Index}

131. The study required analysis of several hundred waves of widely varying forms. Therefore, it was advantageous to use the most consistent and easily applied definition of incipient breaking. Because velocity, acceleration, pressure, and radiation stress were not directly measured in this study, the break point could not be defined by these variables (Singamsetti and Wind 1980). Possible definitions compatible with the capability of the video system and placement of wave gages were (a) the point where the wave cannot adapt to the bottom configuration and begins to disintegrate, (b) the point where the wave height is maximum, (c) and the point where part of the wave front becomes vertical. Several wave gages closely spaced in the vicinity of the break point would be required to accurately define the break point based on maximum wave height. Consequently, it was felt the limited number of wave gages available for the study would be better used to record wave height in 
the surf zone and wave reflection. Use of video equipment allowed each wave to be examined during frame-by-frame playback as waves shoaled up to the break point. The point where the wave front became vertical was selected for use as the break-point definition because it was a unique point that could be readily observed on the video monitor and measured by reference to the grid placed on the side glass.

132. The horizontal location of the break point was a critical parameter because the water depth over the bar became increasingly shallow with small horizontal increments, which made $h_{b}$ and thus breaker depth index sensitive to location. Plunge distance was also measured from the break point; therefore, a change in break-point location results in a different plunge distance. Although the remote sensing system was of high quality, determination of the exact location where the wave front became vertical was subject to judgment. The importance of precisely locating the break point required iteration of frame advancements and retreats during videotape analysis, which became tedious, but once the break point was defined, the breaker height and depth could be obtained with consistency. For some waves the break point occurred between videotape frames. In these cases, the height and depth at breaking were measured from the previous frame. Breaker height and depth were measured from the video monitor using the grid placed on the tank wall as a reference. The 2 -in. grid spacing could be scaled from the monitor to $1 / 4$-in. increments.

133. The breaker depth was measured under the crest of the wave from SWL, which was well defined on the grid. The SWL is the water level that would exist if waves ceased but tide and storm surge remained (in the case of the field), whereas MWL is the time-averaged water surface elevation including the presence of waves. Since tide and storm surge did not exist in the tank, SWL was simply the quiescent water level in the tank. The MWL can easily be obtained from the wave gages; however, breaker depth was not always located at a gage; thus, it was convenient to use SWL as the datum for analyzing the videotapes. Water depth was also measured under the horizontal face of the wave from SWL for comparison with depth under the wave crest. For spilling and plunging waves, the break point was located near the crest, resulting in little difference in $\gamma_{\mathrm{b}}$. However for collapsing waves, the depth at the break point could be up to one-half the depth under the crest, which doubled the value of $\gamma_{b}$. 
134. Breaker heights and depths were determined from videotape for 80 of the 96 total base tests. Measurements of breaker height and depth were made for all tests in which $\beta_{1} \leq 20 \mathrm{deg}$. The values of height and depth represent an average of 10 consecutive waves from each test.

\section{Breaker depth index}

135. Breaker depth index showed considerable scatter if plotted as a function of $\xi_{0}$ (Figure 34 ). Values of $\gamma_{b}$ tend to increase for $\xi_{0}$ $\leq 0.9$, whereas for higher $\xi_{0}$-values the breaker depth index appears to decrease more gently with wide scatter in the range $0.9 \leq \xi_{0} \leq 1.6$, shown by vertical lines. Wave breaking for $\xi_{0}>0.9$ was not only influenced by depth, but by a dependent variable involved in the breaking process, the return flow velocity. Breaking waves at higher $\xi_{0}$-values were typically of the form shown in Figure 35. A secondary wave shoreward of the main wave crest is created by the return flow, which causes the break point to develop

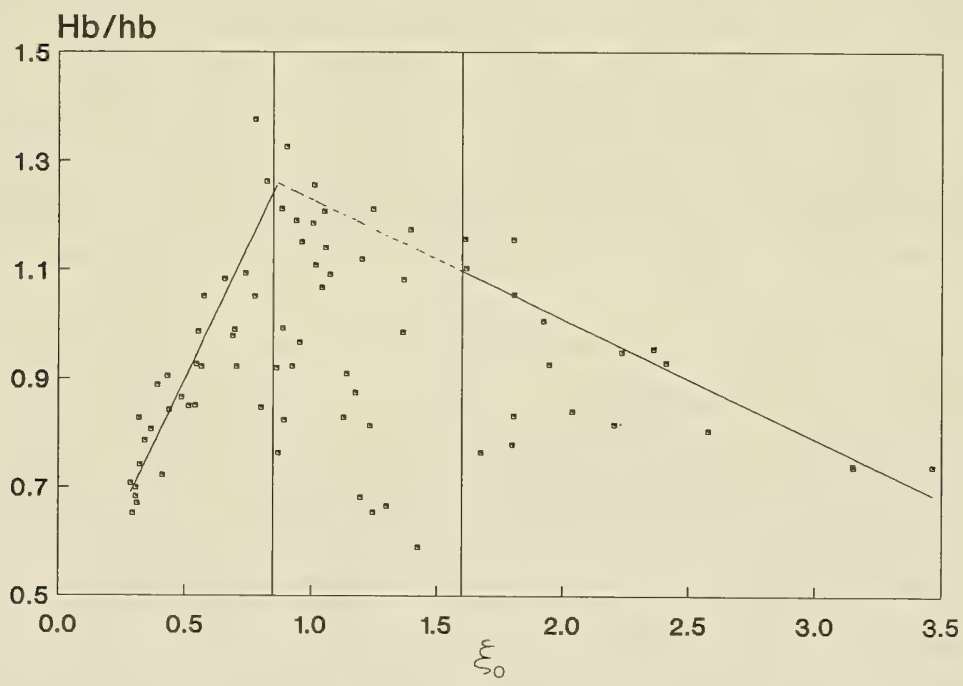

Figure 34. $\gamma_{b}$ as a function of $\xi_{0}$ 


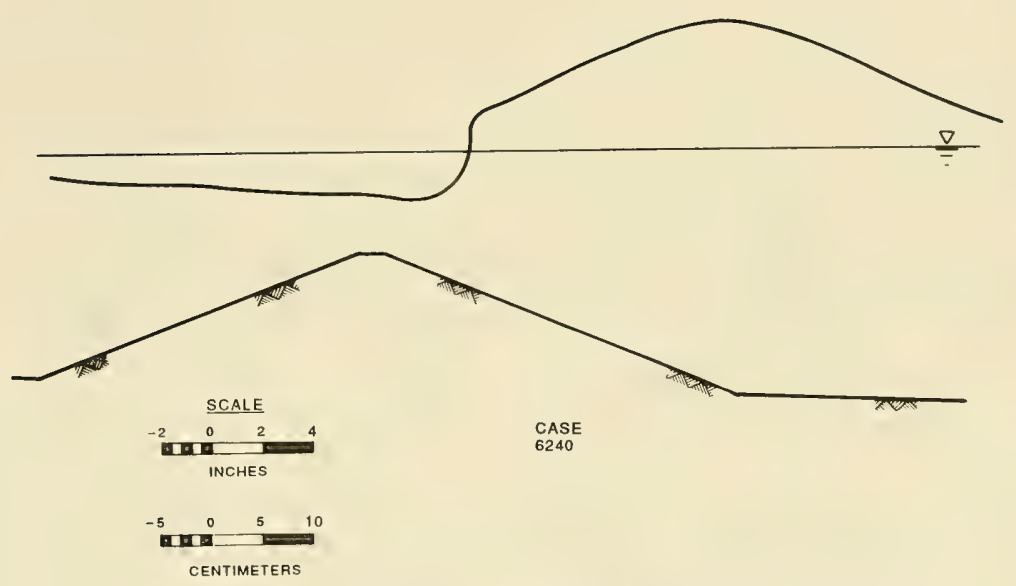

Figure 35. Collapsing wave at incipient breaking

on the front face of the wave crest; i.e., breaking begins before the incident wave has reached the depth-limited break condition. The wave crest was located in deeper water at incipient breaking for these conditions, which increased $h_{b}$ and lowered values of $\gamma_{b}$. The wide scatter of data indicates the strength of the return flow is a major factor influencing breaker depth at higher $\xi_{0}$-values.

136. A breaker depth analysis was made for increasing values and decreasing values of $\gamma_{b}$ in Figure 34, excluding the wide scatter data in the transition region $\left(0.9 \leq \xi_{0} \leq 1.6\right)$. A 1 inear regression was performed on the increasing values of $\gamma_{b}$ for spilling and plunging waves occurring over bars in which $\beta_{1} \leq 10 \mathrm{deg}$, which is approximately the maximum seaward bar angle observed in nature. A relationship for collapsing waves with $\xi_{0} \geq 1.6$ was made by a best-fit line drawn through $\gamma_{b}$-values. The resulting relationships for breaker depth index was:

$$
\begin{aligned}
& \gamma_{b}=0.41+0.98 \xi_{0} \quad \text { for } 0.3 \leq \xi_{0} \leq 0.90 \\
& \gamma_{b}=1.45-0.22 \xi_{0} \text { for } 1.6 \leq \xi_{0} \leq 3.5
\end{aligned}
$$

The coefficient of determination for the regression analysis was 0.85 . Equation 78 is shown in Figure $36 \mathrm{a}$ and $\mathrm{b}$ and also in Figure 34. The decreasing 
relationship of Equation 78 was extended through the transition region, represented by the dashed line in Figure 34, for illustrative purposes. Although the data are widely scattered in this region, the relationship follows the trend of the higher $\gamma_{b}$-values and intersects with the increasing relationship of Equation 78 near the lower limit of the transition region.

137. An exponential relation was also developed to predict $\gamma_{\mathrm{b}}$ for plunging and spilling waves that had $\xi_{0}$-values greater than 0.90 . The visual fit line shown in Figure 37 is expressed as:

$$
\gamma_{\mathrm{b}}=1.3\left(1-e^{-2.5 \xi_{0}}\right)
$$

Equation 79 was obtained for $0.29<\xi_{0}<1.07$ and $\beta_{1} \leq 10 \mathrm{deg}$.

138. Figure 38 gives a comparison of $\boldsymbol{\gamma}_{\mathrm{b}}$ for 5- and 10-deg bar slopes computed using Equation 79 to the equation developed for plane slopes, Equation 58, as a function of deepwater wave steepness. The prediction for planar slopes exceeds the limits of beach slope given for Equation 58. The values shown by the plane slope equation are values for $m=1 / 10$. Equation 58 gives higher values of $\gamma_{\mathrm{b}}$ than Equation 79 for $\beta_{1}=5 \mathrm{deg}$ for $0.02<\mathrm{H}_{\circ} / \mathrm{L}_{\circ}$ $<0.09$, but underpredicts $\gamma_{\mathrm{b}}$ for $\beta_{1}=10 \mathrm{deg}$. Also shown in Figure 38 are the expected values of $\gamma_{b}$ on a 1/30 slope using Equation 58, which predicts $\gamma_{\mathrm{b}}$ slightly lower for high wave steepness for $\beta_{1}=5 \mathrm{deg}$. Equation 58 significantly underpredicts $\gamma_{\mathrm{b}}$ for low wave steepnesses for $\beta_{1}=5 \mathrm{deg}$ and for all wave steepnesses for $\beta_{1}=10 \mathrm{deg}$.

Breaker height index

139. Values of $\Omega_{\mathrm{b}}$ as a function of deepwater wave steepness are shown in Figure 39 (a-d) grouped by seaward bar angle. The data show increasing breaker height with decreasing wave steepness, which is also typical of wave breaking on a plane slope. Therefore, it is reasonable to express the data collected on barred profiles in the same form as the relationships developed for plane-slope data:

$$
\Omega_{\mathrm{b}}=C\left(\beta_{1}\right)\left(\frac{\mathrm{H}_{\mathrm{o}}}{\mathrm{L}_{\mathrm{o}}}\right)^{-\mathrm{n}\left(\beta_{1}\right)}
$$




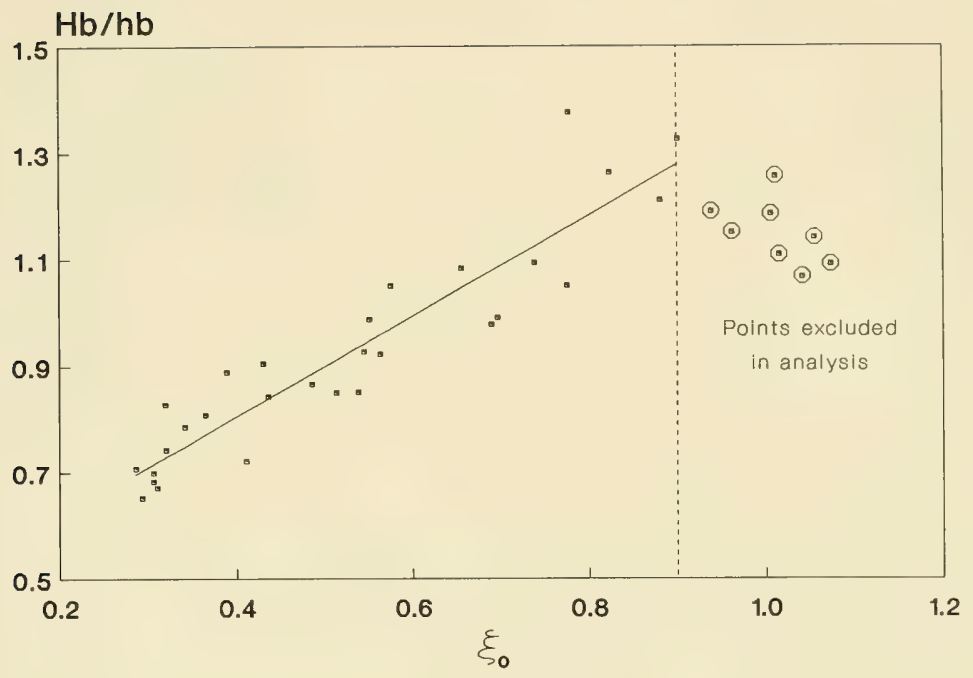

a. Spilling and plunging waves

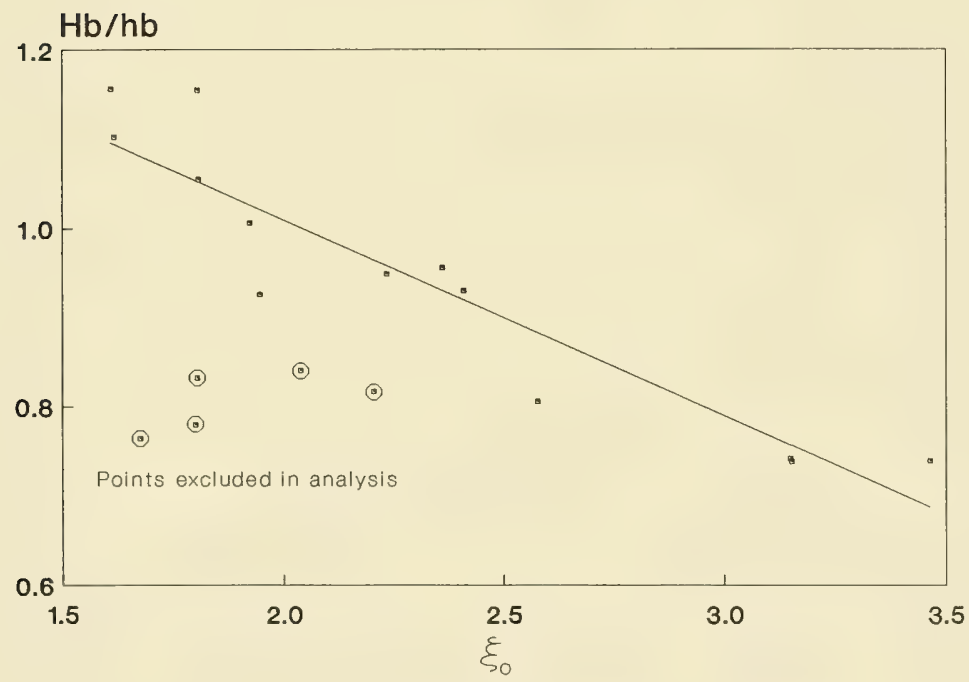

b. Collapsing waves

Figure 36. Expression of $\gamma_{b}$ as a function of $\xi_{0}$ 


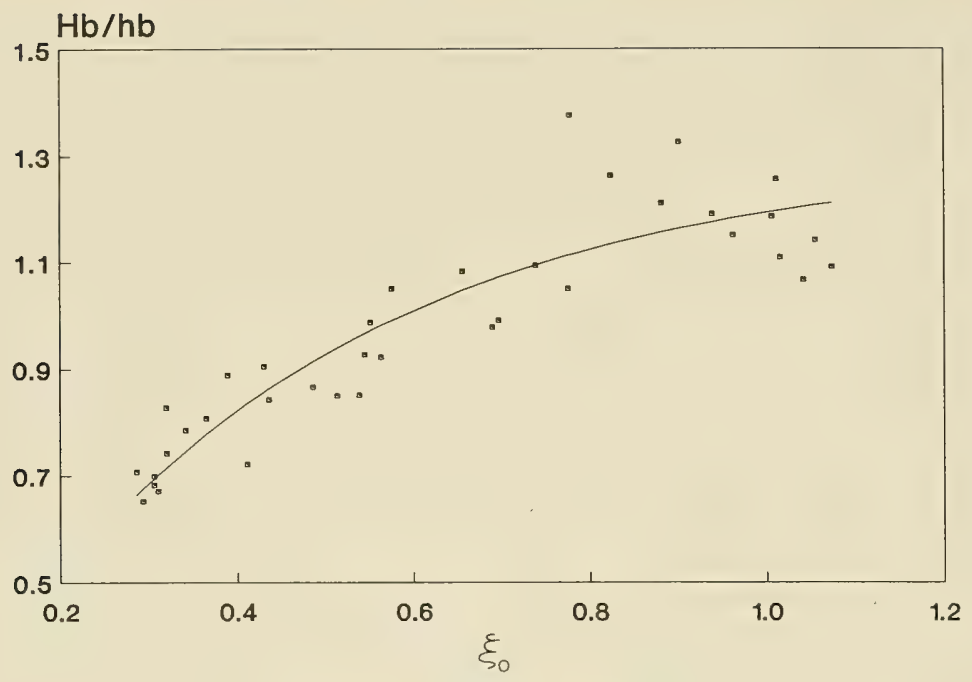

Figure 37. Exponential expression of $\gamma_{b}$ as a function of $\xi_{\text {o }}$

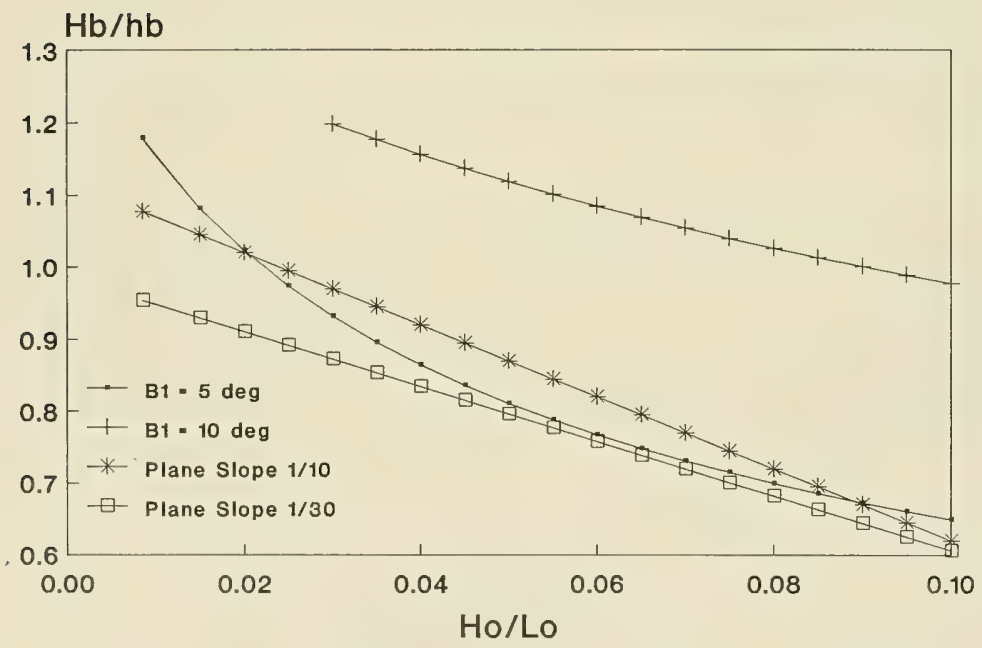

Figure 38. Comparison of breaker depth relationships developed for barred profiles and plane slopes 


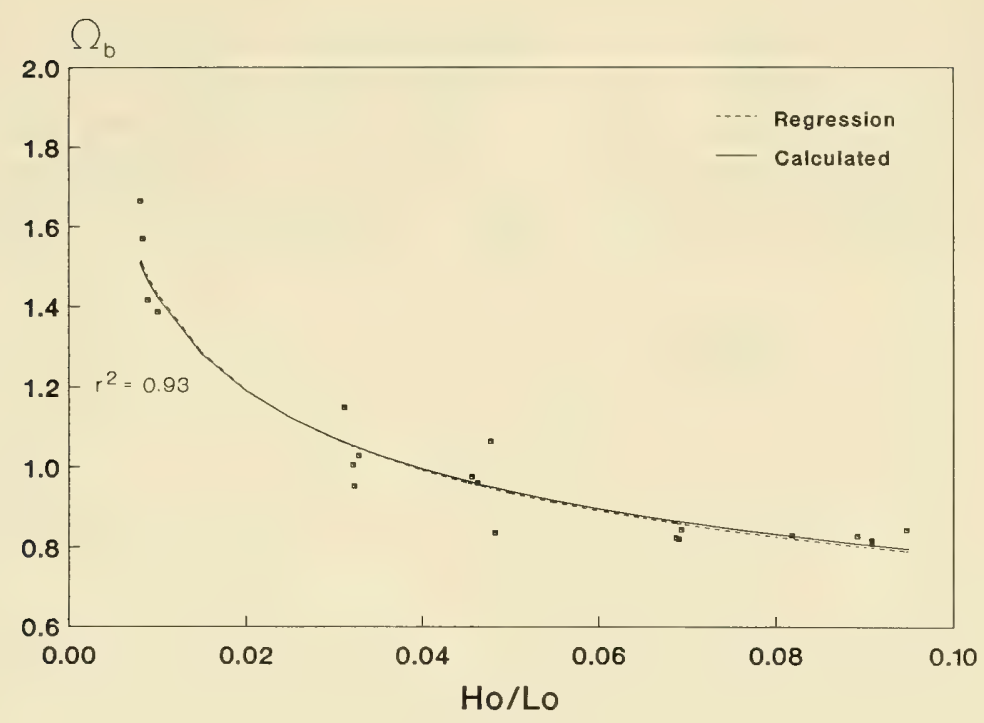

a. $\beta_{1}=5 \mathrm{deg}$

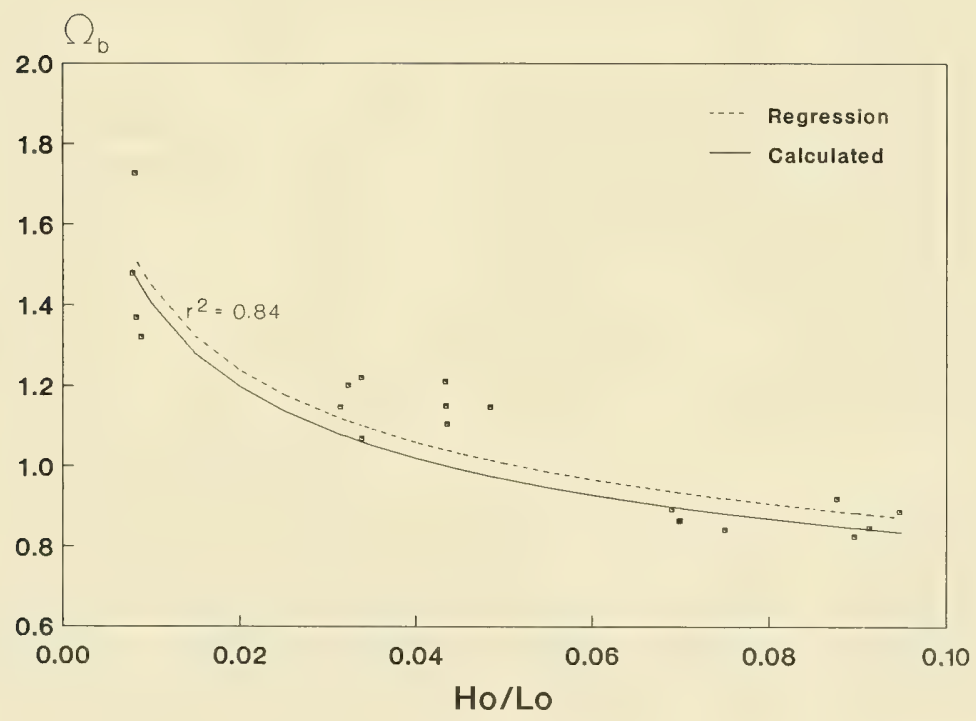

b. $\beta_{1}=10 \mathrm{deg}$

Figure 39. $\Omega_{\mathrm{b}}$ as a function of $\mathrm{H}_{\mathrm{o}} / \mathrm{L}_{\mathrm{o}}$. (Continued) 


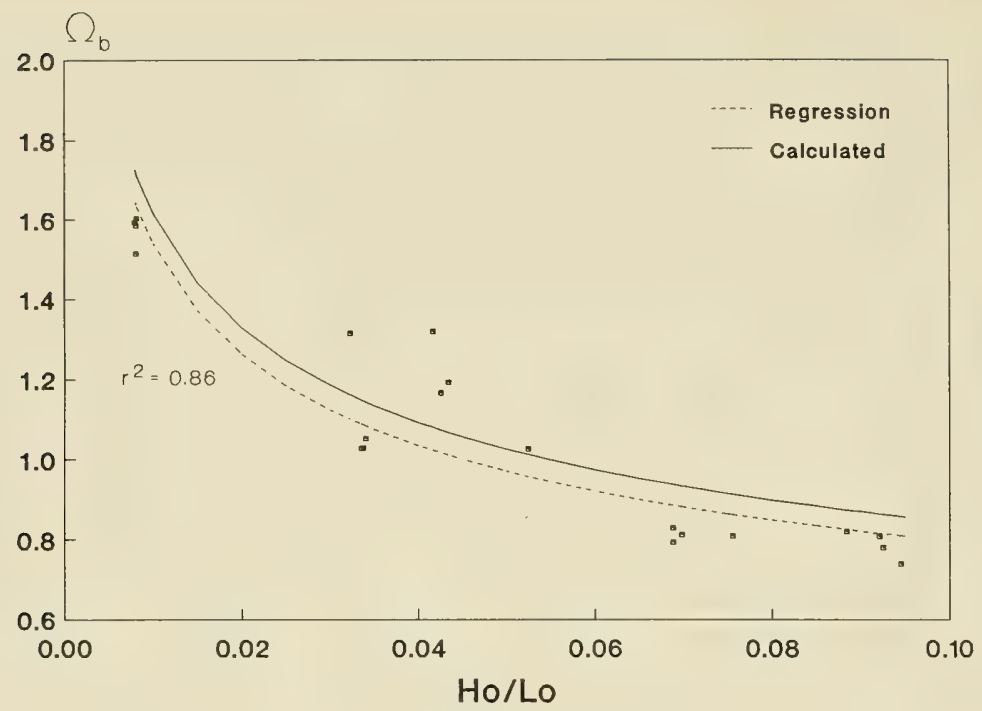

c. $\beta_{1}=15 \mathrm{deg}$

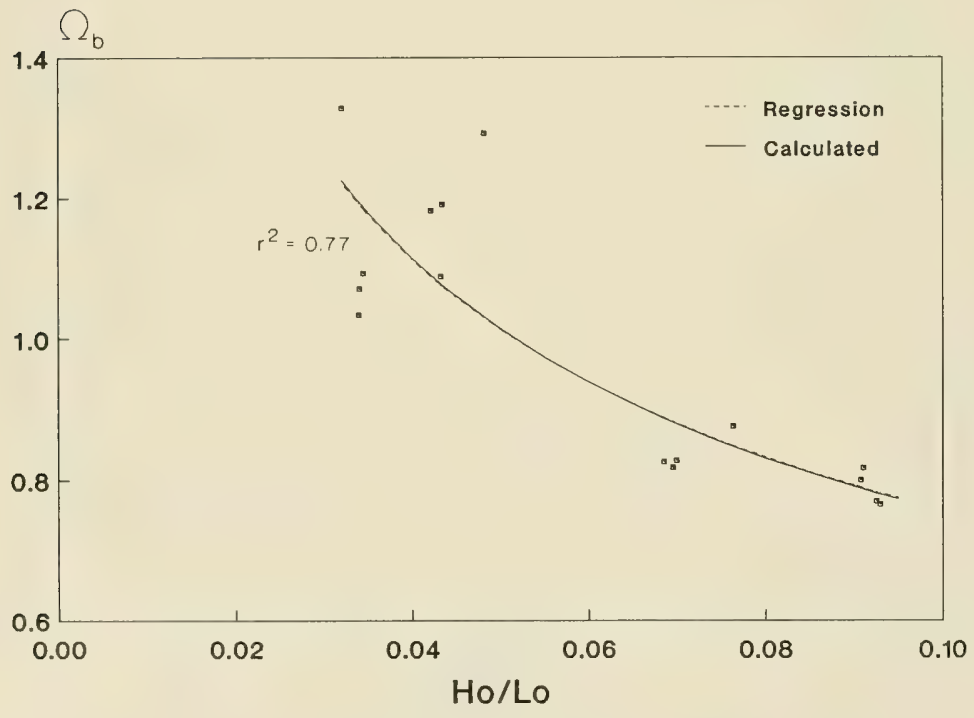

d. $\beta_{1}=20 \mathrm{deg}$

Figure 39. (Concluded) 
An equation in the form of Equation 80 was developed for each seaward bar angle by performing a regression analysis on $\Omega_{b}$ as a function of $H_{0} / L_{o}$, represented by the dashed line in Figure 39 (a-d). Equations for the empirical parameters $\mathrm{C}\left(\beta_{1}\right)$ and $\mathrm{n}\left(\beta_{1}\right)$ were obtained by plotting these two parameters as a function of seaward bar angle, Figures 40 and 41, and fitting an equation to the data by regression analysis. The resulting polynomial functions were

$$
C\left(\beta_{1}\right)=0.28+2.17 \tan \beta_{1}-6.00 \tan ^{2} \beta_{1}
$$

and

$$
\mathrm{n}\left(\beta_{1}\right)=0.36-1.59 \tan \beta_{1}+4.85 \tan ^{2} \beta_{1}
$$

The coefficients of determination for $\mathrm{C}\left(\beta_{1}\right)$ and $\mathrm{n}\left(\beta_{1}\right)$ were 0.93 and 0.99 , respectively. The calculated values of $\Omega_{\mathrm{b}}$ using Equation 80 are shown as the solid line in Figure $39(\mathrm{a}-\mathrm{d})$. The variation of breaker height index calculated from the regression analysis of each $\beta_{1}$-angle is presented in Figure 42 as a function of wave steepness. Figure 43 shows a comparison of $\Omega_{\mathrm{b}}$ by seaward angle using Equation 80 .

140. Figure 42 shows that $\Omega_{\mathrm{b}}$ decreases for values of $\beta_{1}>10 \mathrm{deg}$ for high wave steepnesses, whereas for low wave steepnesses $\Omega_{b}$ becomes larger. Since the steeper bars are shorter, shoaling of the incident wave prior to breaking occurs mainly on the $1 / 30$ plane slope rather than the bar, and $\Omega_{\mathrm{b}}$ is approximately the same as the values predicted for the $1 / 30$ slope using Equation 61. Figure 43 shows $\Omega_{\mathrm{b}}$-values for the 15 -deg bar are higher than those for the 10-deg bar at higher wave steepnesses, which differs from Figure 42. This is simply due to the variability in the regression of the coefficients $\mathrm{C}\left(\beta_{1}\right)$ and $\mathrm{n}\left(\beta_{1}\right)$.

141. The higher values of $\Omega_{\mathrm{b}}$ observed at low wave steepnesses may be explained by the presence of a strong return flow visually observed in these cases. A heuristic explanation can be given to describe the influence of return velocity on wave height. The treatment is based on the depth integrated energy equation of Phillips (1977), which for the situation of the tank becomes: 


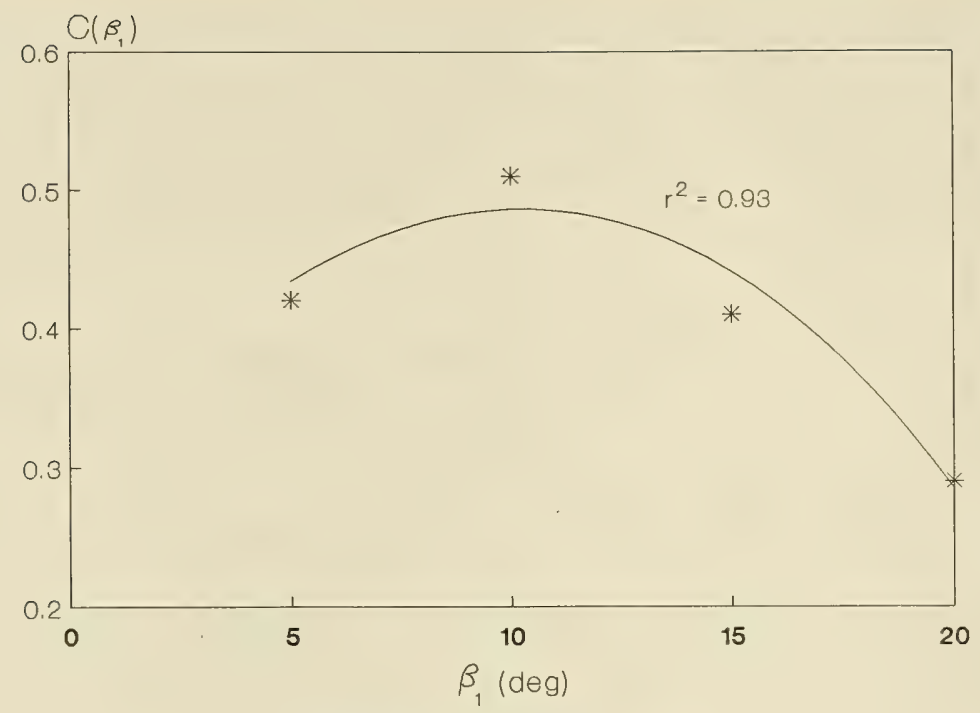

Figure 40. Function $\mathrm{C}\left(\beta_{1}\right)$

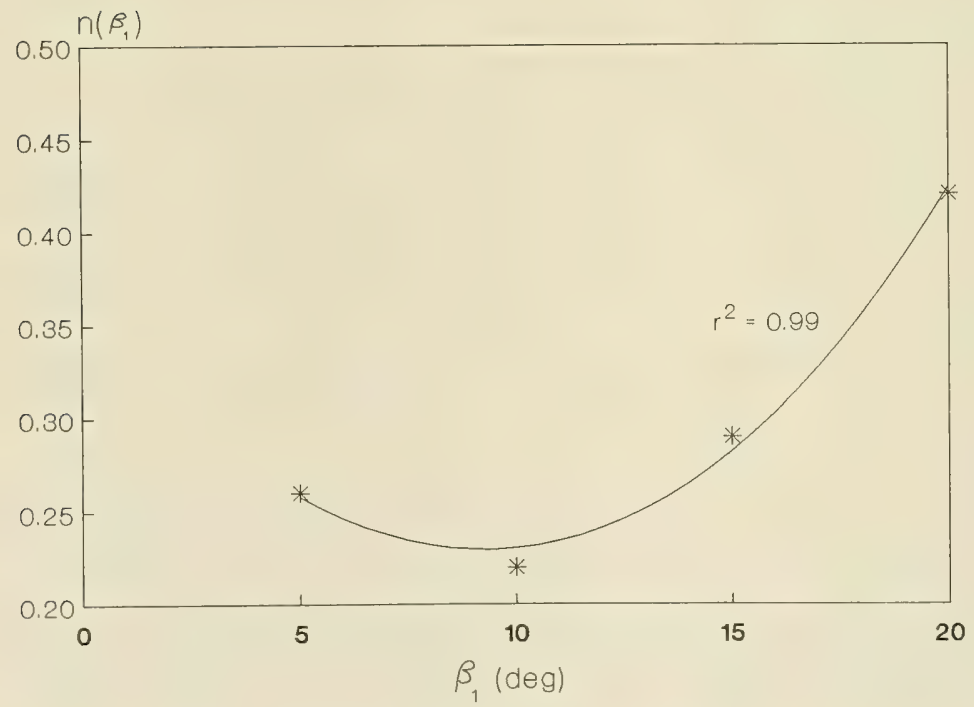

Figure 41. Function $\mathrm{n}\left(\beta_{1}\right)$ 


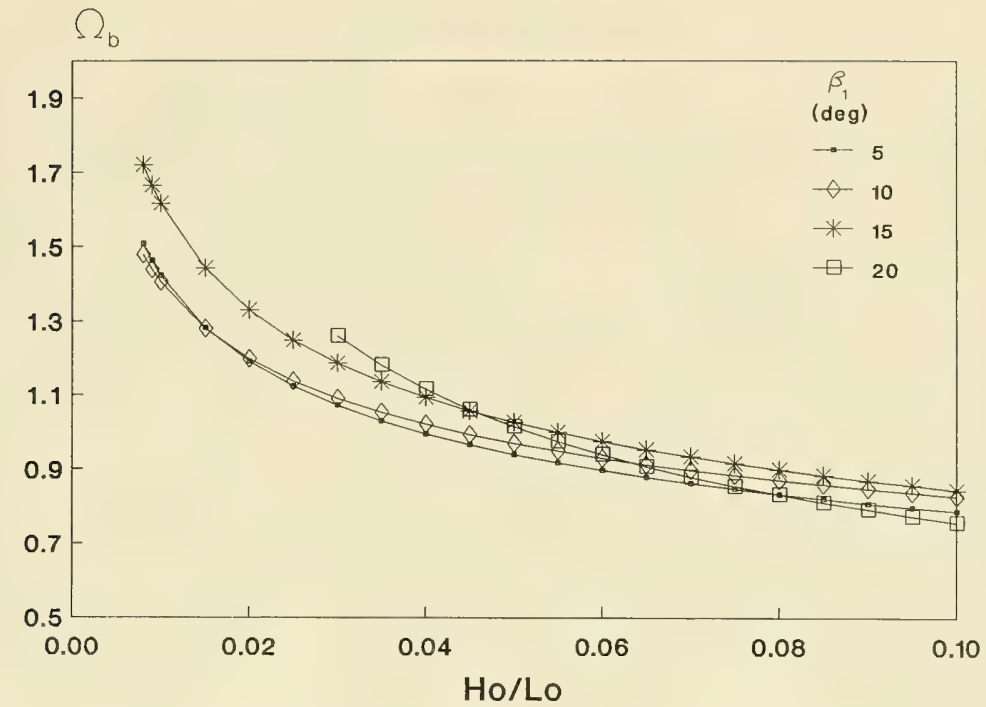

Figure 42. Regression curves of $\Omega_{b}$ as a function of $H_{o} / L_{o}$

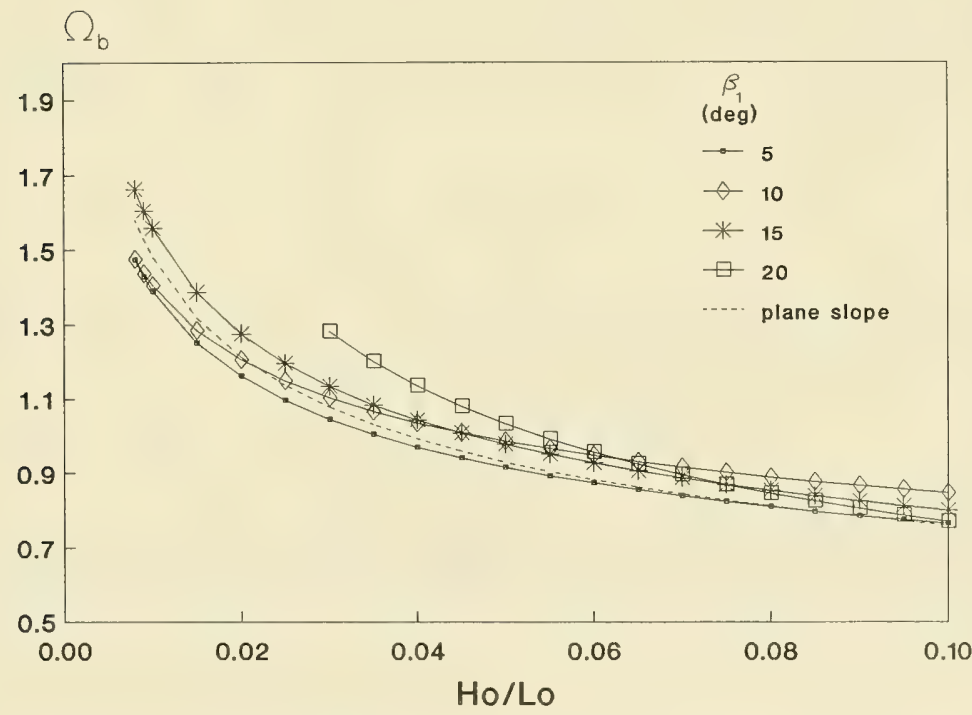

Figure 43. Calculated values of $\Omega_{b}$ as a function of $H_{0} / L_{\circ}$ 


$$
\frac{d}{d x}\left[E\left(U+C_{g}\right)\right]+S_{x x} \frac{d U}{d x}=0
$$

in which $U$ is the horizontal flow speed taken positive in the shoreward direction and $S_{x x}$ is the shoreward component of the radiation stress. The return flow $U$ is created to balance the mass of water thrown shoreward in the crest of the breaking wave. Equation 83 will be evaluated at the end of the tank near the wave board (called deep water) and at the break point. To simplify the discussion the term $S_{x x} d U / d x$ will be omitted, although it is non-negligible. Also, frictional losses in the tank, expected to be small, are neglected.

142. At the wave board, $U$ must equal zero since the current cannot penetrate through the board. In the open ocean, this is equivalent to $U$ being zero in deep water. With this consideration and the stated assumptions, Equation 83 gives

$$
\left[E\left(-U+C_{g}\right)\right]_{b}^{\prime}=\left[E C_{g}\right]_{0}
$$

in which the prime denotes conditions where return flow is present and the minus sign in front of the magnitude $U$ indicates flow in the seaward direction. If no return flow or only a very small flow is present, Equation 84 is simply:

$$
\left[\mathrm{EC}_{\mathrm{g}}\right]_{\mathrm{b}}=\left[\mathrm{EC}_{\mathrm{g}}\right]_{0}
$$

Equating the different breaking conditions (with and without a return flow) having identical deepwater wave conditions yields

$$
\left[E\left(-U+C_{g}\right)\right]_{b}^{\prime}=\left[E_{g}\right]_{b}
$$

Substitution of the equation for wave energy density (Equation 39) into Equation 86 and elimination of constants on both sides of the resultant equation yields the following expression:

$$
\left(\mathrm{H}_{\mathrm{b}}^{\prime}\right)^{2}\left(\mathrm{C}_{\mathrm{gb}}^{\prime}-\mathrm{U}_{\mathrm{b}}\right)=\mathrm{H}_{\mathrm{b}}^{2} \mathrm{C}_{\mathrm{gb}}
$$


where

$\mathrm{H}_{\mathrm{b}}^{\prime}=$ breaking wave height in presence of return flow

$\mathrm{C}_{\mathrm{gb}}^{\prime}=$ group speed of waves at breaking in presence of return flow

$\mathrm{U}_{\mathrm{b}}=$ magnitude of the return flow in the vicinity of the break point Assuming $\mathrm{C}_{\mathrm{gb}}^{\prime} \approx \mathrm{C}_{\mathrm{gb}}$, Equation 87 can be expressed as:

$$
\left(\frac{\mathrm{H}_{\mathrm{b}}^{\prime}}{\mathrm{H}_{\mathrm{b}}}\right)^{2}=\frac{1}{1-\frac{\mathrm{U}_{\mathrm{b}}}{\mathrm{C}_{\mathrm{gb}}}}
$$

Under the stated assumptions, Equation 88 demonstrates that a return flow produces higher breaking wave heights than if the flow is absent. Figure $44 a$ and b shows $\Omega_{\mathrm{b}}$ as a function of deepwater wave steepness for seaward angles of 5 and $20 \mathrm{deg}$. The highest $\Omega_{\mathrm{b}}$-values result from conditions in which the cross-sectional water area shoreward of the bar was $\operatorname{small}\left(\beta_{3}=0 \mathrm{deg}\right)$ or the shoreward angle was very steep $\left(\beta_{3}=40 \mathrm{deg}\right)$. Return flow increases for $\beta_{3}=0$ deg because of continuity; i.e., velocity increases if the water area shoreward of the bar decreases. For situations with steep shoreward slopes, the vertical component of return flow velocity is directed opposite (up) to that expected for a plane slope (down). For cases in which the weir flow was observed, steep $\beta_{1}$-angles and low wave steepness, the return flow speed increased over the crest of the bar. Equation 88 shows that under these circumstances $H_{b}$ will increase, which may explain the increase in $\Omega_{b}$-values for 20-deg angles at low values of wave steepness in Figures 42 and 43 .

143. The preceding derivation shows that a stronger return flow increases the breaker height, but observations indicate $\Omega_{b}$ is still well behaved in the presence of strong return flow, unlike the breaker depth index. This suggests breaker height is a more stable parameter than breaker depth for situations with steep bar faces. The derivation can be refined by including the wave-current interaction term $\mathrm{S}_{\mathrm{xx}} \mathrm{dU} / \mathrm{dx}$ and actual values of $\left(\mathrm{C}_{\mathrm{g}}\right)_{\mathrm{b}}$ and $\left(\mathrm{C}_{\mathrm{g}}\right)_{\mathrm{b}}$, leading to an equation that must be solved by iteration. 


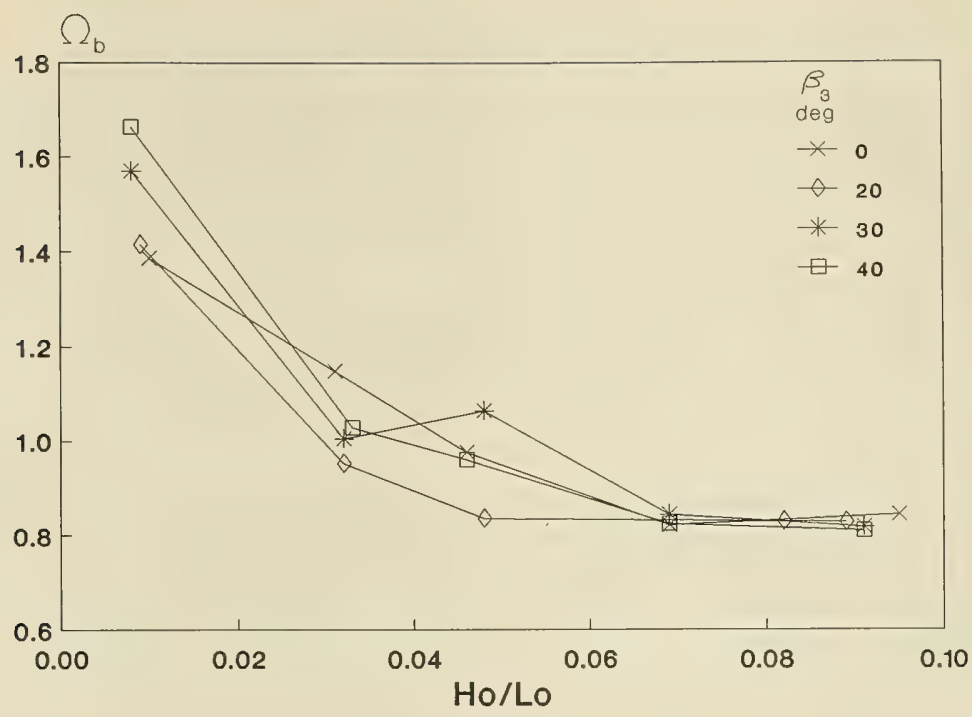

a. $\beta_{1}=5 \mathrm{deg}$

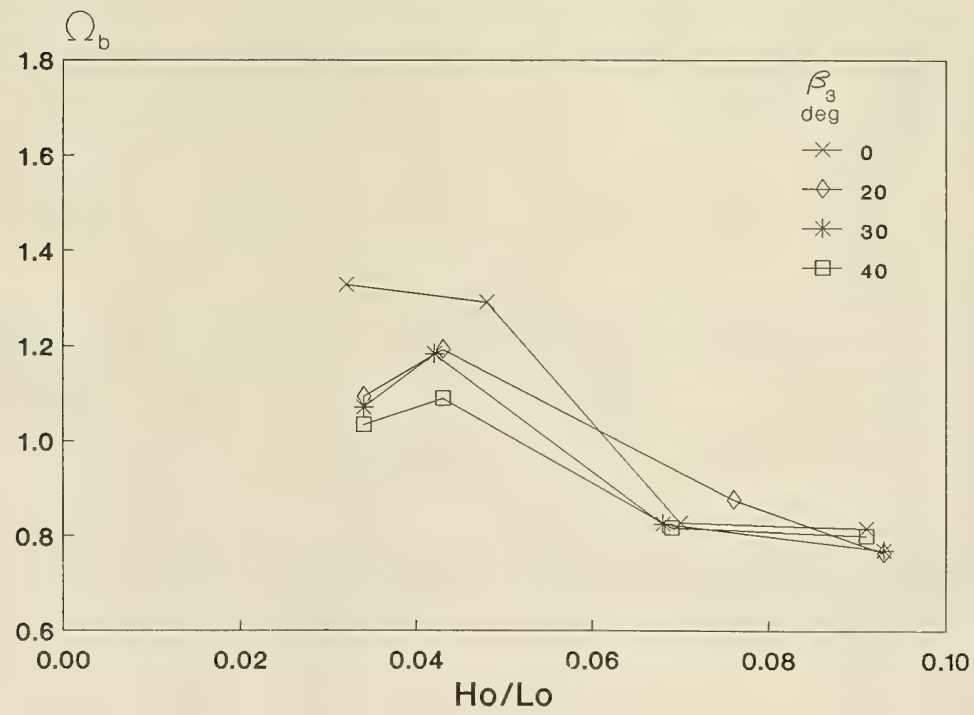

b. $\beta_{1}=20 \mathrm{deg}$

Figure 44. $\Omega_{b}$ as a function of $H_{0} / L_{0}$ 


\section{Plunge Distance}

144. After measurements of breaker height, breaker depth, and breaking location were made for monochromatic wave tests, the videotape was advanced to the plunge point, and plunge distance was measured. Plunge distances were averaged for the same waves that breaker height and depth were measured. Occasionally, the plunge point was obstructed from the video camera by tank wall supports. In these cases, the plunge distance was averaged for the number of unobstructed observations that could be made. Figure 45 shows plunge distance normalized by breaking wave height as a function of $\xi_{0}$. The solid line drawn through the data by visual fit represents the following equation:

$$
\frac{\mathrm{X}_{\mathrm{p}}}{\mathrm{H}_{\mathrm{b}}}=0.63 \xi_{\mathrm{o}}^{-1.00}+1.81
$$

for $0.29 \leq \xi_{0} \leq 3.46$.

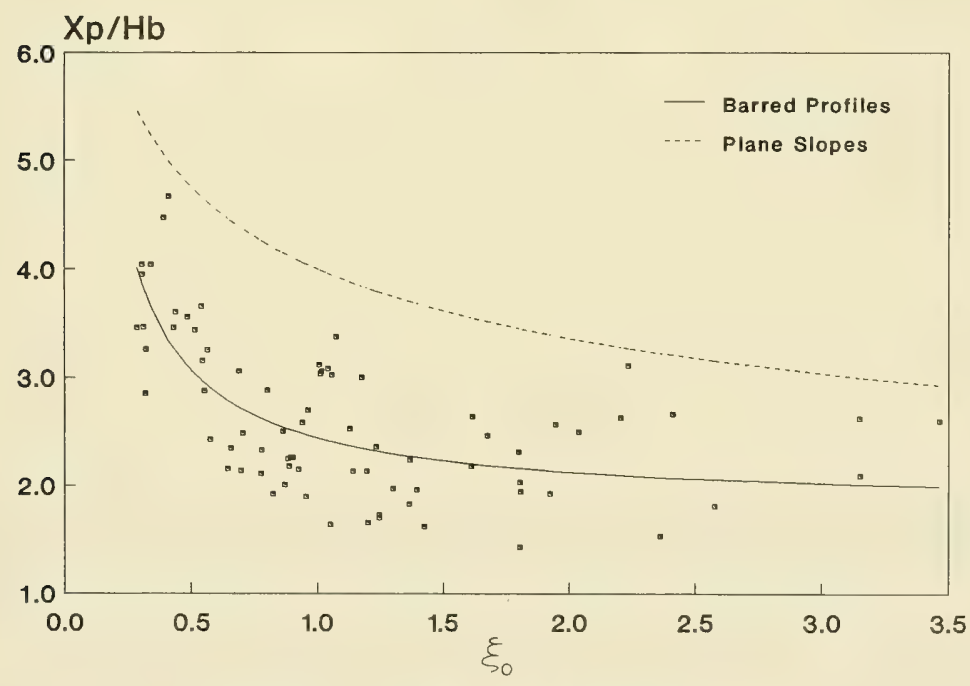

Figure 45. $\mathrm{X}_{\mathrm{p}} / \mathrm{H}_{\mathrm{b}}$ as a function of $\xi_{\text {o }}$ 
145. Plunge distance was also plotted as a function of the local surf similarity parameter (Figure 46). The visual-fit solid line through the data is expressed as

$$
\frac{\mathrm{X}_{\mathrm{p}}}{\mathrm{H}_{\mathrm{b}}}=0.65 \xi_{\mathrm{b}}^{-1.05}+1.80
$$

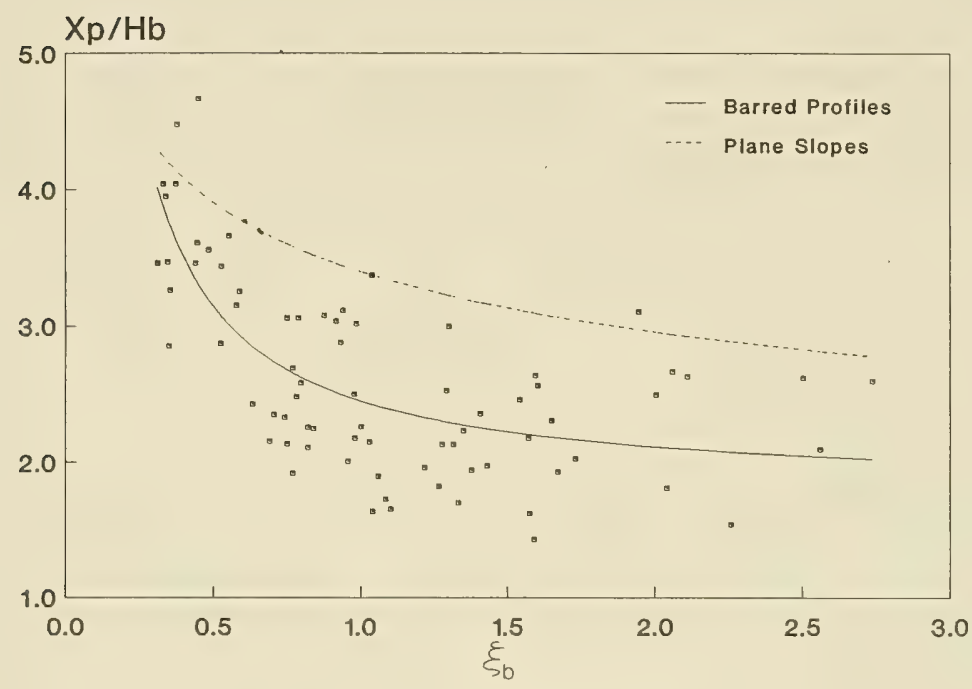

Figure 46. $\mathrm{x}_{\mathrm{p}} / \mathrm{H}_{\mathrm{b}}$ as a function of $\xi_{\mathrm{b}}$

for $0.31 \leq \xi_{\mathrm{b}} \leq 2.74$, where $\xi_{\mathrm{b}}$ is an inshore surf similarity parameter. Both Figures 45 and 46 show comparable scatter of the data, and neither Equation 89 nor 90 appears to be a better predictor of plunge distance.

146. The equations developed in Part II for plunge distance on plane slopes is shown by the dashed line in Figures 45 and 46 . Plunge distance for barred profiles are shorter than those for plane slopes with common values of $\xi_{0}$. This can be explained by the transformation of breaker type caused by the barred profiles. Spilling and plunging waves would be expected for the range of $\xi$-values shown in Figures 45 and 46, whereas spilling, plunging, and collapsing waves occurred for the barred profiles. The data obtained for barred profiles indicate that $\mathrm{X}_{\mathrm{p}} / \mathrm{H}_{\mathrm{b}}$ decreases steeply for smaller surf 
similarity values and approaches a constant value for larger surf similarity parameter values, whereas plunge distance on plane slopes shows a gradual decrease with increasing surf similarity parameter. This could also be an effect of the return flow, since return flow was observed to be stronger at larger $\xi_{0}$-values.

147. Figure 47 shows no apparent trend for plunge distance normalized by deepwater wave height plotted as a function of $\xi_{0}$, which means that plunge distance is insensitive to deepwater wave height. A representative value visually determined from Figure 47 is $x_{p} \approx 3 H_{o}$.

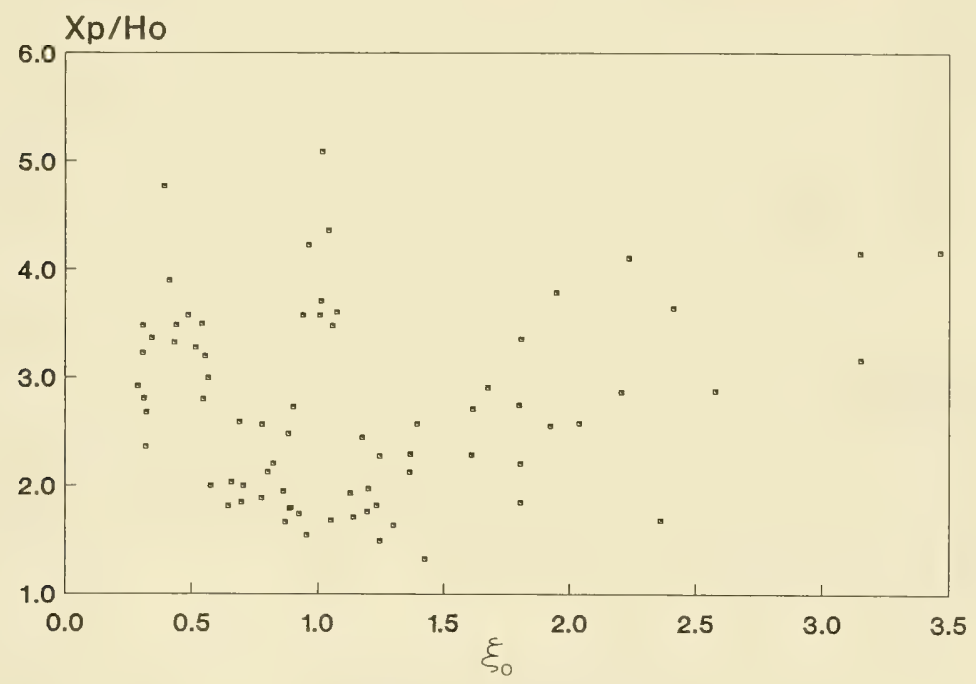

Figure 47. $\mathrm{X}_{\mathrm{p}} / \mathrm{H}_{\mathrm{o}}$ as a function of $\xi_{0}$

\section{Splash Distance}

148. The procedure used to measure plunge distance was also applied to measure splash distance. Splash distance normalized by $H_{b}$ as a function of $\xi_{0}$ is shown in Figure 48. The data are extremely scattered and show no trend. The splash distance is evidently not dependent on breaking wave height.

149. The ratio of plunge distance to splash distance ranged from 0.6 to 2.7 , but average plunge distance was approximately equal to the splash distance $\left(\mathrm{X}_{\mathrm{p}} / \mathrm{X}_{\mathrm{s}}=0.95\right)$, which agrees with the result of Galvin (1968) for 


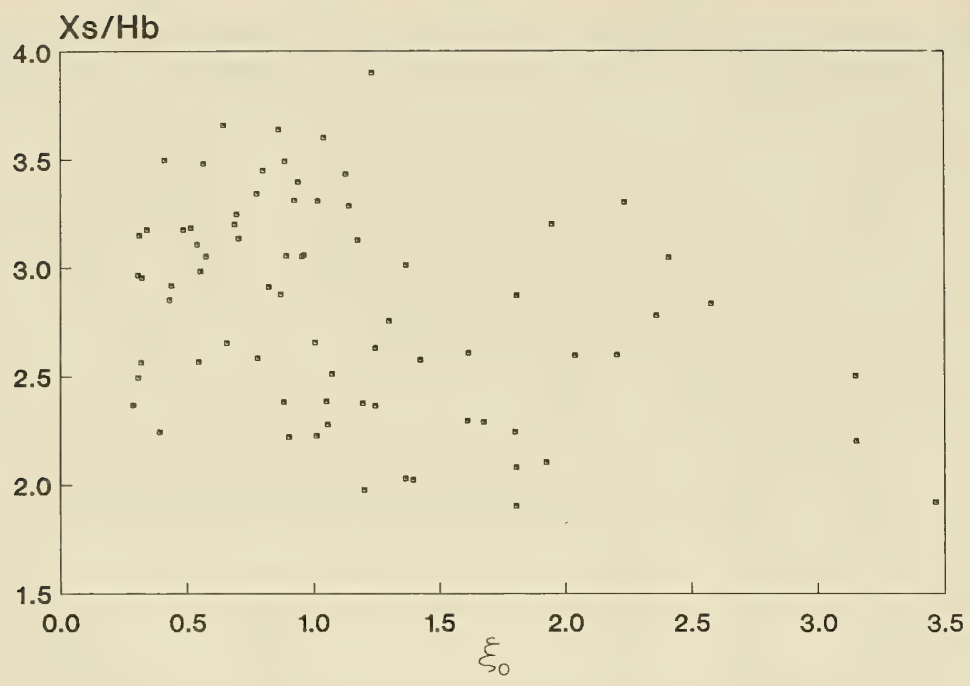

Figure 48. $\mathrm{X}_{\mathrm{s}} / \mathrm{H}_{\mathrm{b}}$ as a function of $\xi_{0}$

plane-sloped beaches. Values of $\mathrm{X}_{\mathrm{p}} / \mathrm{X}_{\mathrm{s}}$ tended to be greater for smaller $\xi_{0}$-values as shown in Figure 49. Plunge distance was also longer for smaller values of $\xi_{0}$, which may explain this trend. The dashed line in Figure 49 represents the empirical relation of Galvin (1968), and the solid line is given by the following visually fit equation

$$
\frac{X_{p}}{X_{s}}=0.14 \xi_{o}^{-1.50}+0.70
$$

for $0.29 \leq \xi_{0} \leq 3.46$. Figure 50 shows $X_{p} / X_{s}$ as a function of $\xi_{b}$, with the empirical equation of Galvin shown by the dashed line. The solid line was visually fit to the data and is identical to Equation 91 with $\xi_{\mathrm{b}}$ substituted for $\xi_{0}$, for $0.31 \leq \xi_{\mathrm{b}} \leq 2.74$. Equation 91 may be rewritten as: 


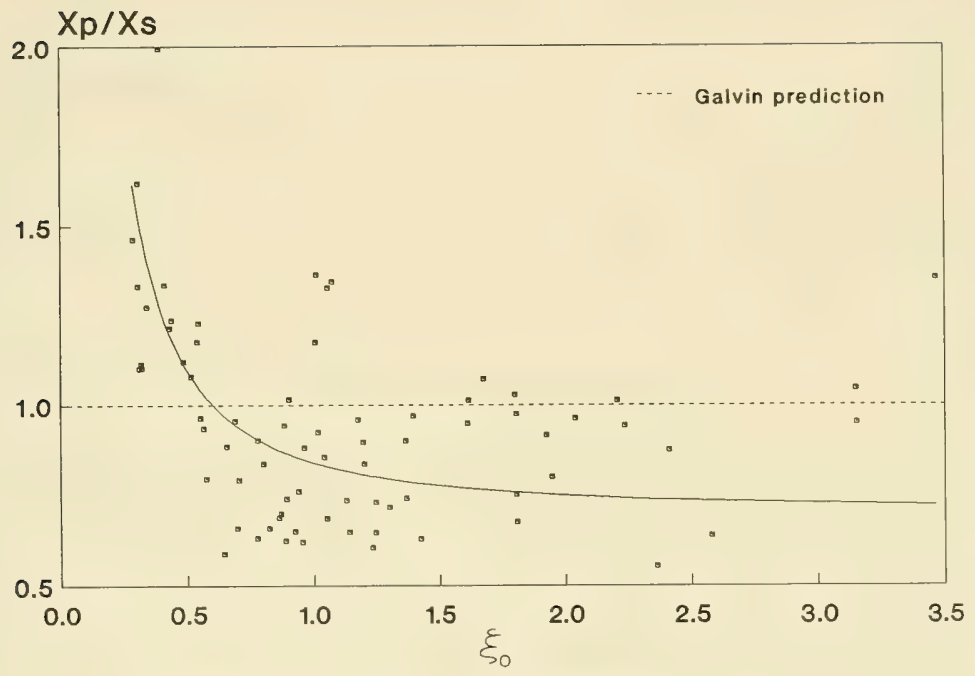

Figure 49. $\mathrm{X}_{\mathrm{p}} / \mathrm{X}_{\mathrm{s}}$ as a function of $\xi_{0}$

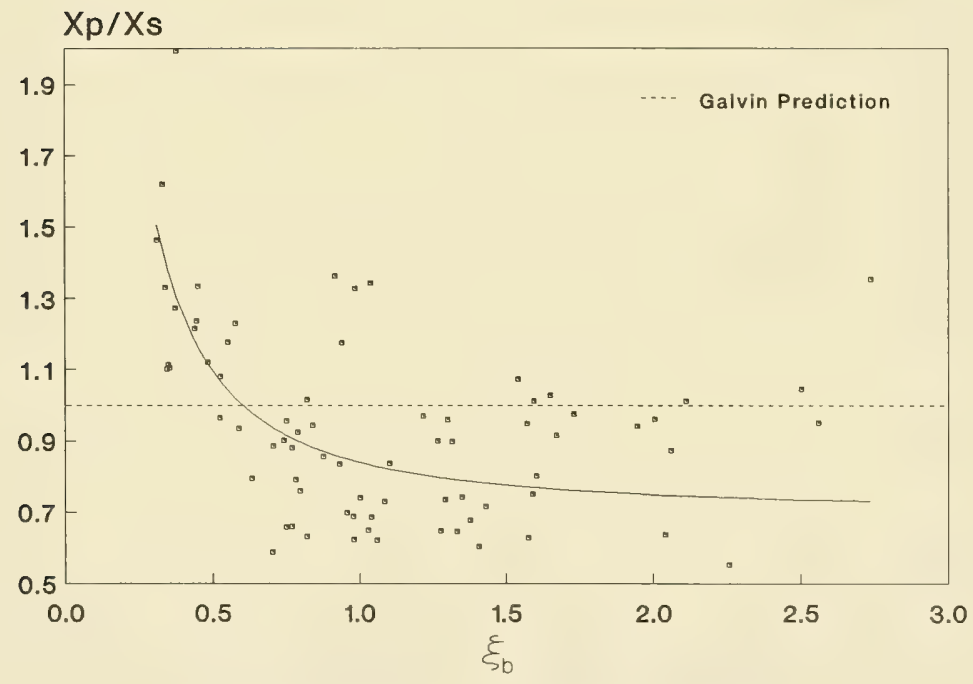

Figure 50. $\mathrm{X}_{\mathrm{p}} / \mathrm{X}_{\mathrm{s}}$ as a function of $\xi_{\mathrm{b}}$ 


$$
\frac{X_{p}}{X_{s}}=0.14 \xi_{b}^{-1.50}+0.70
$$

This implies that either surf similarity parameter may be used. Equations 91 and 92 give good indications of the trend; however, variability in the data is large. From observations of the video recordings, splash distance appeared to be a function of not only breaker type, but also of the angle at which the plunging wave entered the water column.

\section{Penetration Distance}

150. A wave crest continues to travel shoreward upon entering the water column until its forward momentum ceases. The penetration distance $\mathrm{x}_{\mathrm{t}}$ was defined as the total travel distance of the wave crest from the break point. Values of $x_{t}$ were estimated from videotape by observing the maximum forward travel of bubbles caused by the turbulence of the wave crest plunging into the water column. Figure 51 shows penetration distance normalized by breaking

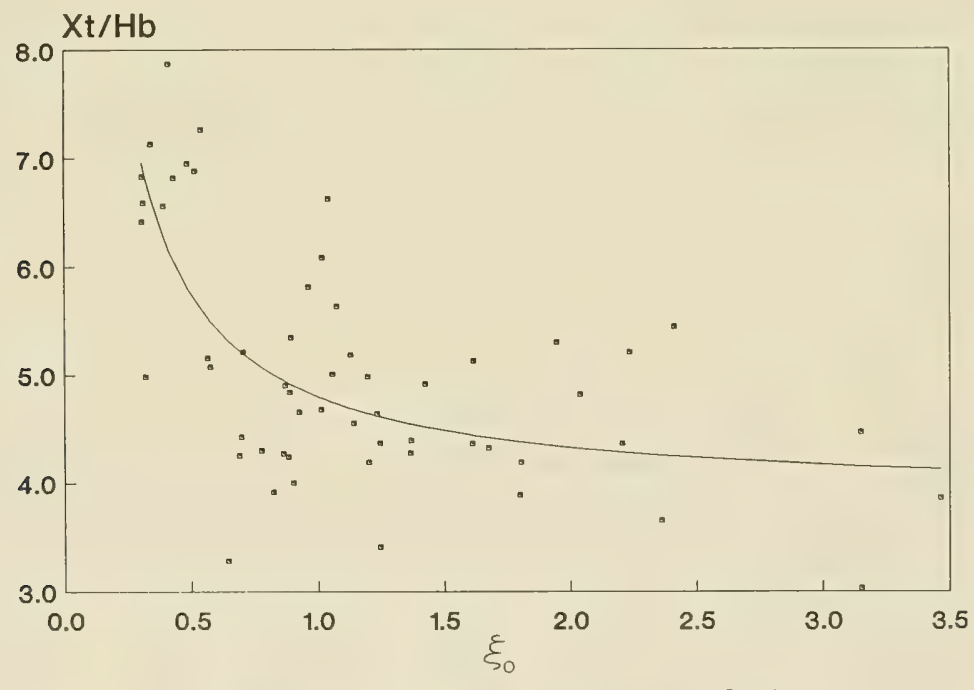

Figure 51. $\mathrm{X}_{\mathrm{t}} / \mathrm{H}_{\mathrm{b}}$ as a function of $\xi_{0}$ 
wave height as a function of the surf similarity parameter. Data obtained from terraced bar cases were not included in analysis because the shoreward face of the bar hindered forward travel of the wave crest. The line shown in Figure 51 is given by

$$
\frac{X_{t}}{H_{b}}=0.95 \xi_{o}^{-1}+3.85
$$

with limits of $0.31 \leq \xi_{0} \leq 3.46$. The expression for $\mathrm{X}_{\mathrm{t}} / \mathrm{H}_{\mathrm{b}}$ as a function of the local surf similarity parameter shown in Figure 52 is

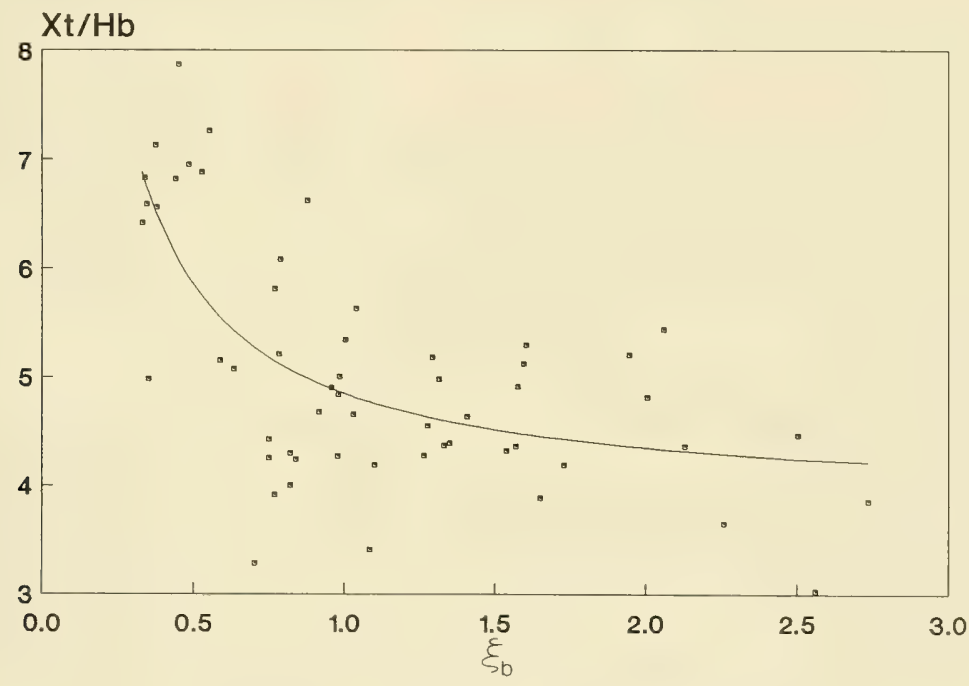

Figure 52. $\mathrm{X}_{\mathrm{t}} / \mathrm{H}_{\mathrm{b}}$ as a function of $\xi_{\mathrm{b}}$

$$
\frac{X_{t}}{H_{b}}=1.00 \xi_{b}^{-1}+3.85
$$

for $0.33 \leq \xi_{\mathrm{b}} \leq 2.74$. Both Equations 93 and 94 were determined by visual fit. Penetration distance normalized by $H_{0}$ shows much scatter if plotted versus $\xi_{0}$ (Figure 53). The hypothesis made that $x_{p}$ is primarily dependent on local wave height also applies to penetration distance. 


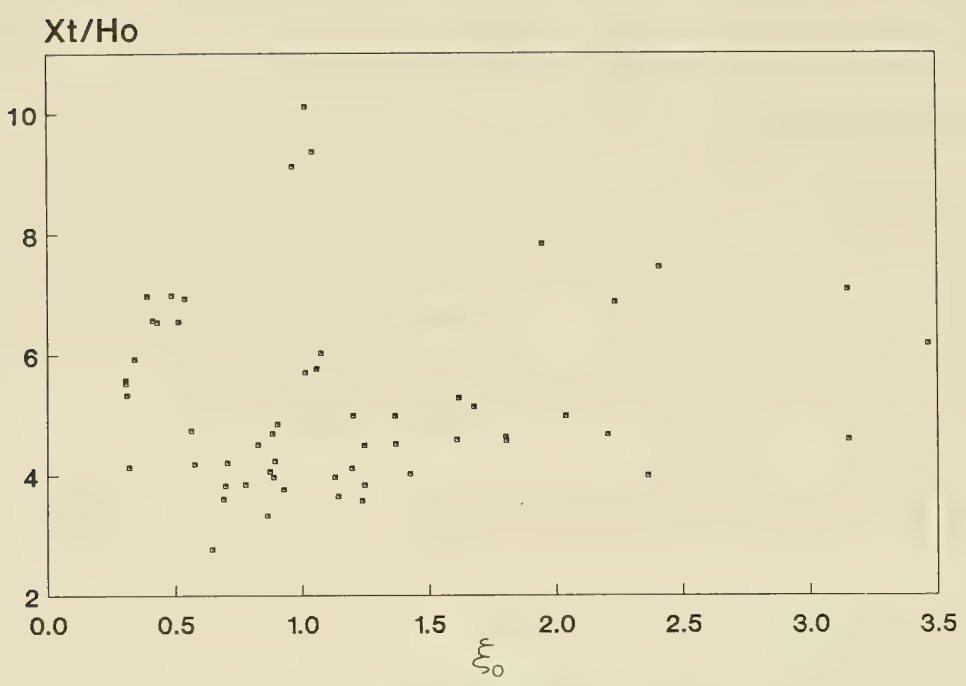

Figure 53. $\mathrm{X}_{t} / \mathrm{H}_{\mathrm{o}}$ as a function of $\xi_{\mathrm{o}}$

\section{Breaker Vortex}

151. The breaker vortex is formed when air becomes entrapped by the overturning crest at the plunge point. As the vortex penetrates the water column, the angular velocity of the vortex decreases and the cross-sectional area $A_{v}$ of the vortex becomes greater, until the energy is dissipated. Because the vortex area increases as the vortex moves into the water column, measurements of $A_{v}$ were made at a distinct location. The plunge point is unique to all breaking waves and is also the location of vortex formation; therefore, $A_{v}$ was measured at this point. The shape of the vortex was elliptical in most cases (Figure 7); the major and minor axes of the vortex were measured, and the equation for an ellipse was used to estimate vortex area .

152. Vortex area was estimated for 34 tests with seaward angles less than $10 \mathrm{deg}$. Vortex area normalized by deepwater wave height was plotted as a function of $\xi_{0}$ in Figure 54, which shows $A_{v} / H_{0}^{2}$ is small for spilling breakers and larger for plunging and collapsing breakers. Although only three cases in the collapsing range were available for analysis, the data show the trend represented by the visually fit equation 


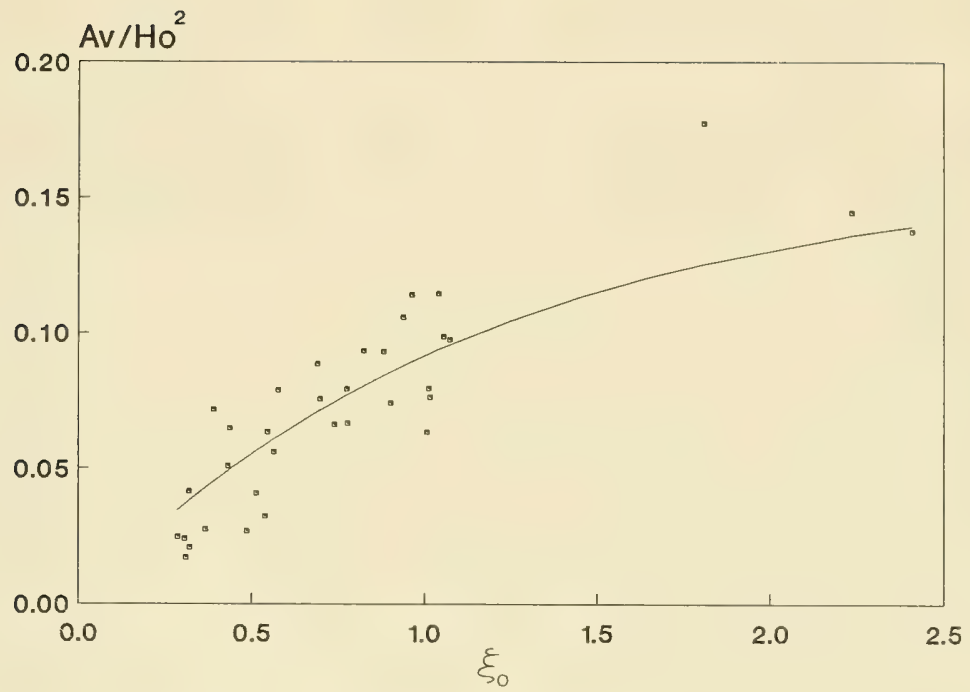

Figure 54. Exponential relation for $A_{v} / H_{0}^{2}$ as a function of $\xi$ 。

$$
\frac{\mathrm{A}_{\mathrm{v}}}{\mathrm{H}_{\mathrm{o}}^{2}}=0.16\left(1-e^{-0.85 \xi_{0}}\right)
$$

for $0.29 \leq \xi_{0} \leq 2.06$. A linear regression was also made for spilling and plunging waves only, shown in Figure 55, which gave:

$$
\frac{\mathrm{A}_{\mathrm{v}}}{\mathrm{H}_{\mathrm{o}}^{2}}=0.005+0.089 \xi_{0}
$$

The coefficient of determination was 0.69 , and the equation is limited to $0.29 \leq \xi_{0} \leq 1.07$. Vortex area was also plotted versus $\xi_{\mathrm{b}}$, Figure 56. The line drawn through the data represents 


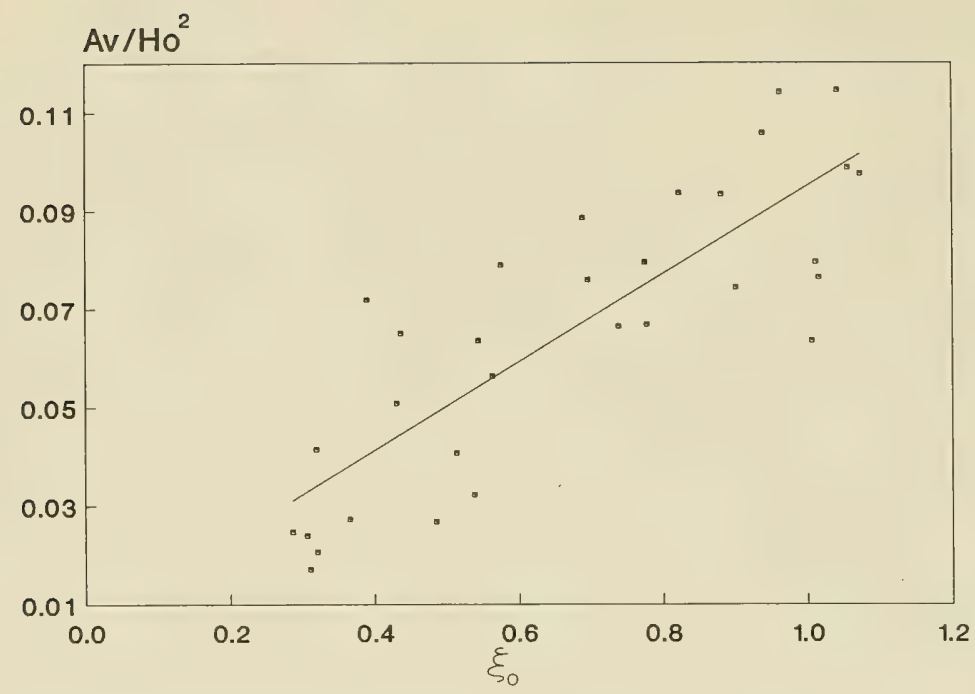

Figure 55. Linear relation for $\mathrm{A}_{v} / \mathrm{H}_{\mathrm{o}}^{2}$ as a function of $\xi_{0}$

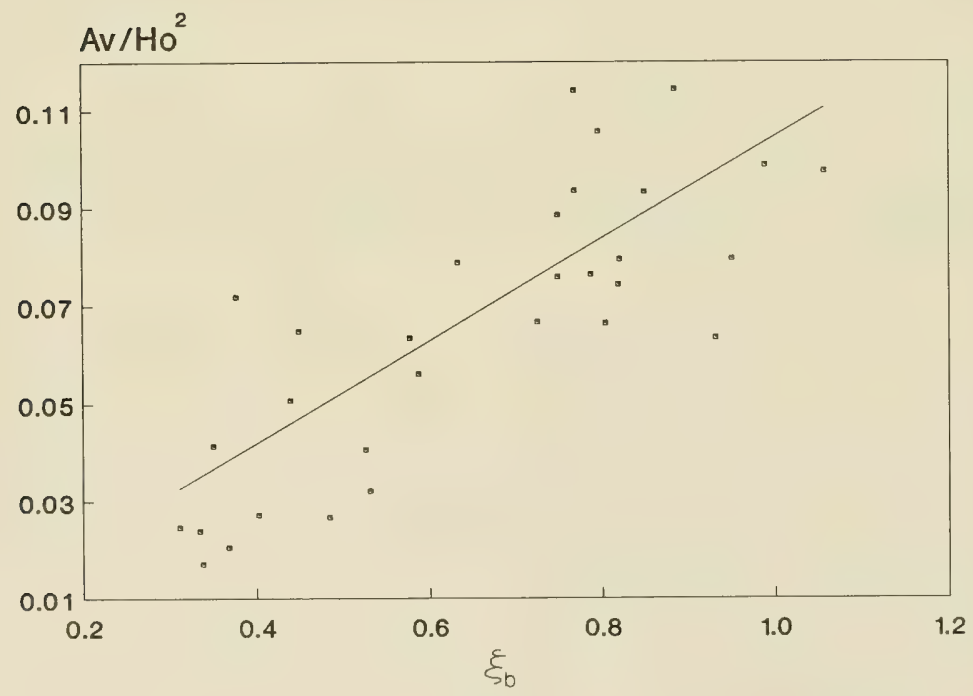

Figure 56. Linear relation for $\mathrm{A}_{\mathrm{v}} / \mathrm{H}_{\mathrm{o}}^{2}$ as a function of $\xi_{\mathrm{b}}$ 


$$
\frac{\mathrm{A}_{\mathrm{v}}}{\mathrm{H}_{\mathrm{o}}^{2}}=0.105 \xi_{\mathrm{b}}
$$

for $0.31 \leq \xi_{\mathrm{b}} \leq 1.06$. Equations 96 and 97 give good estimates of vortex area for spilling and plunging waves. For larger values of the surf similarity parameter, Equation 95 can be used to estimate $A_{v}$.

\section{Wave Height Decay}

153. Wave height measurements were made for all tests, and the data should be of value for surf zone wave model development. Figure $57(a-e)$ shows wave height at each gage normalized by $\mathrm{H}_{\circ}$ as a function of distance for five cases. Comparison of measured wave height with predictions of wave decay models is left for future work. Because beach profiles were irregular, numerical models (e.g., Svendsen (1984, 1985); Dally (1980); and Dally, Dean, and Dalrymple (1985a, 1985b)) will need to be applied. Average wave heights for 12 to 15 waves at each gage for all tests are included in Appendix C.

\section{Wave Runup}

154. Wave runup measurements were made for all tests. Figure 58 shows $\mathrm{R} / \mathrm{H}_{0}$ plotted as a function of seaward bar angle $\beta_{1}$, and, for the planeslope data, beach slope angle $\left(m=1 / 30\right.$, i.e., $\left.\beta_{1}=1.91 \mathrm{deg}\right)$. Except for the plane-slope points, each data point represents the average $\mathrm{R} / \mathrm{H}_{\mathrm{o}}-\mathrm{value}$ for the four shoreward bar angles associated with the seaward bar angle for the particular wave steepness. Basically, the seaward bar angle has no influence on runup. Runup also shows no dependence on $\xi_{0}$, as shown in Figure 59. Runup was plotted versus the surf similarity parameter using the 1/30 slope as the primary angle. The data show increasing $\mathrm{R} / \mathrm{H}_{\mathrm{o}}$ with increasing values of $\xi_{0}$. The line drawn in Figure 60 represents the average value of $\left(\mathrm{R} / \mathrm{H}_{\mathrm{o}}\right) / \xi_{0}$, which was 0.76 , whereas Equation 52 (Battjes 1975) predicts this ratio to be 1.0. Figure 61 shows runup for the plane slope cases versus the surf similarity parameter. All measured values are overpredicted by Equation 52, shown as the solid line. The cause of the difference in runup determined in this experiment and by Battjes is not known; additional measurements are clearly warranted. 


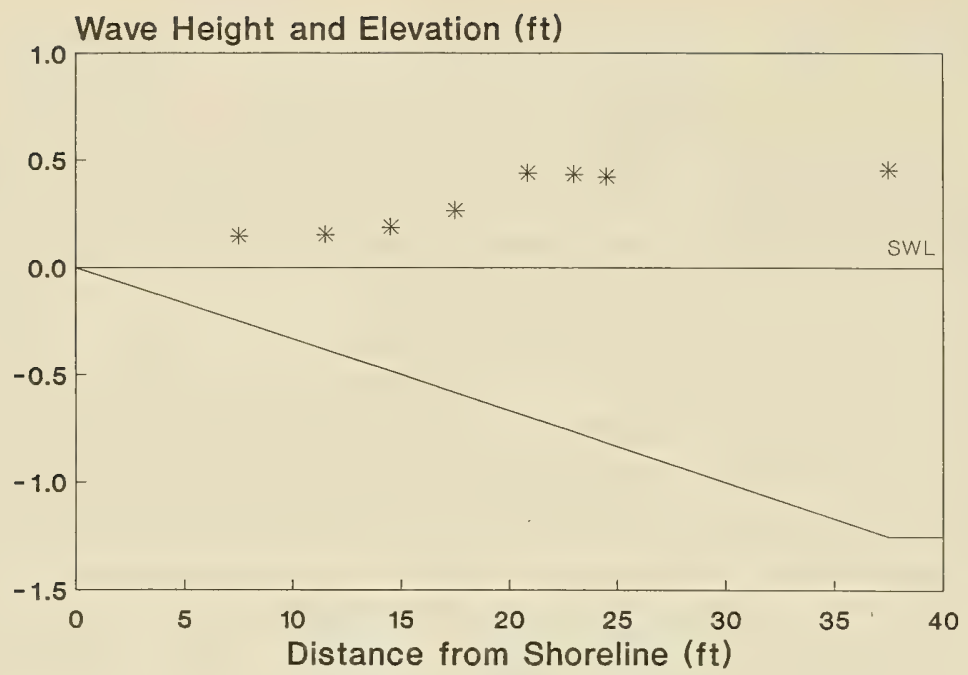

a. Case 2000

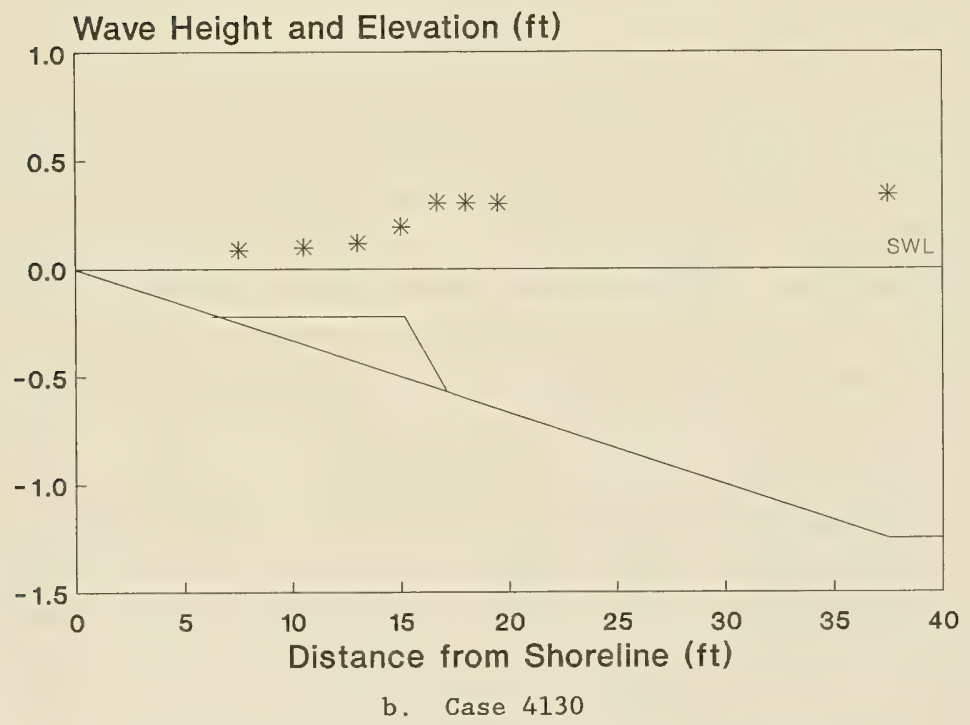

Figure 57. Wave height as a function of horizontal distance (Sheet 1 of 3 ) 

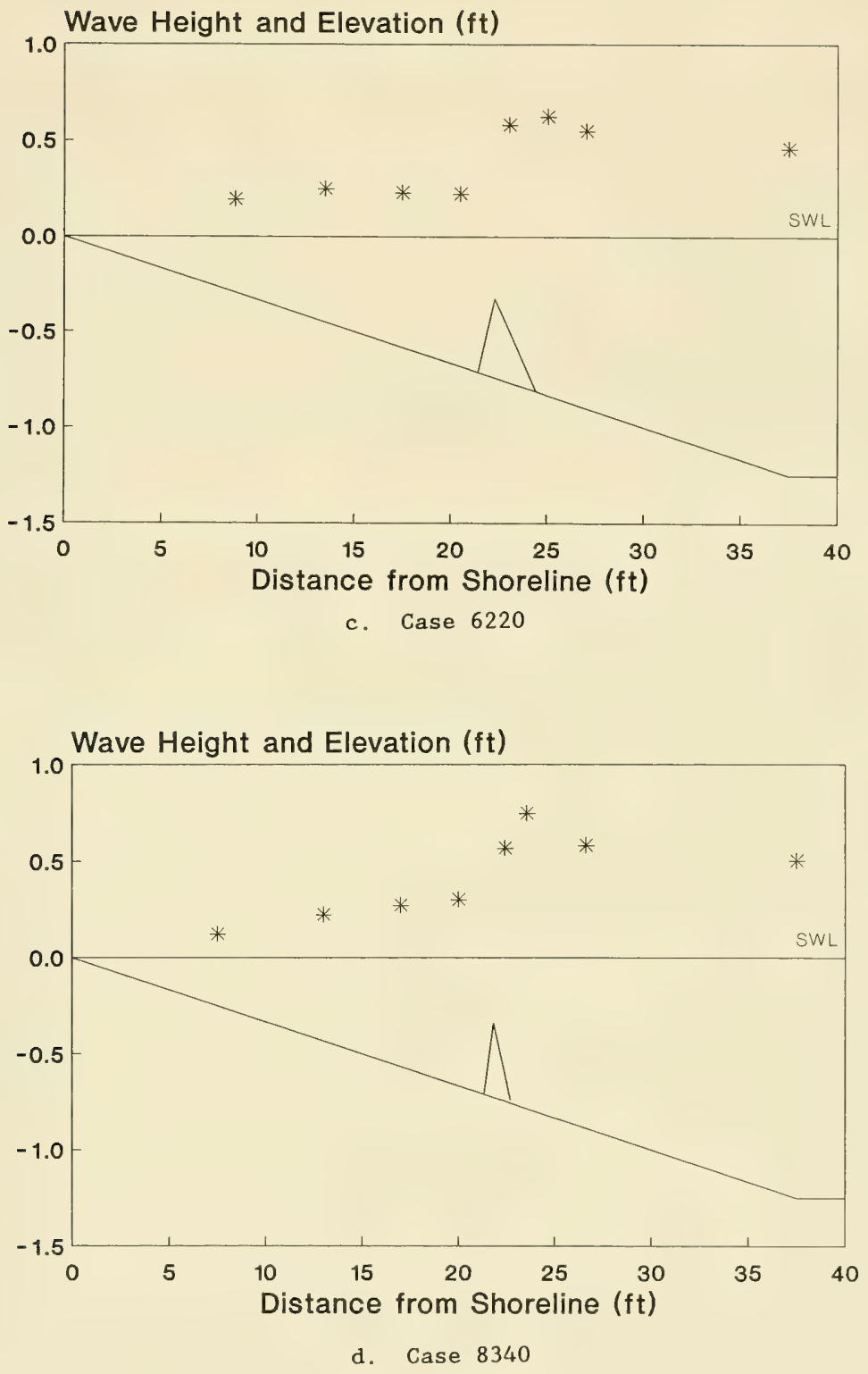

Figure 57 . (Sheet 2 of 3 ) 


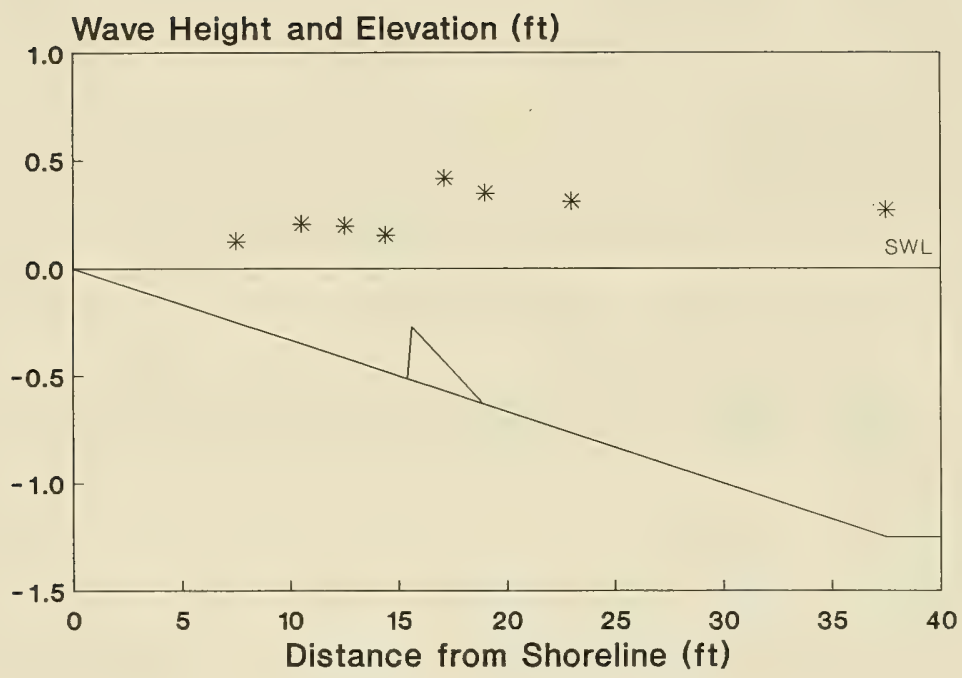

e. Case 10410

Figure 57. (Sheet 3 of 3 ) 


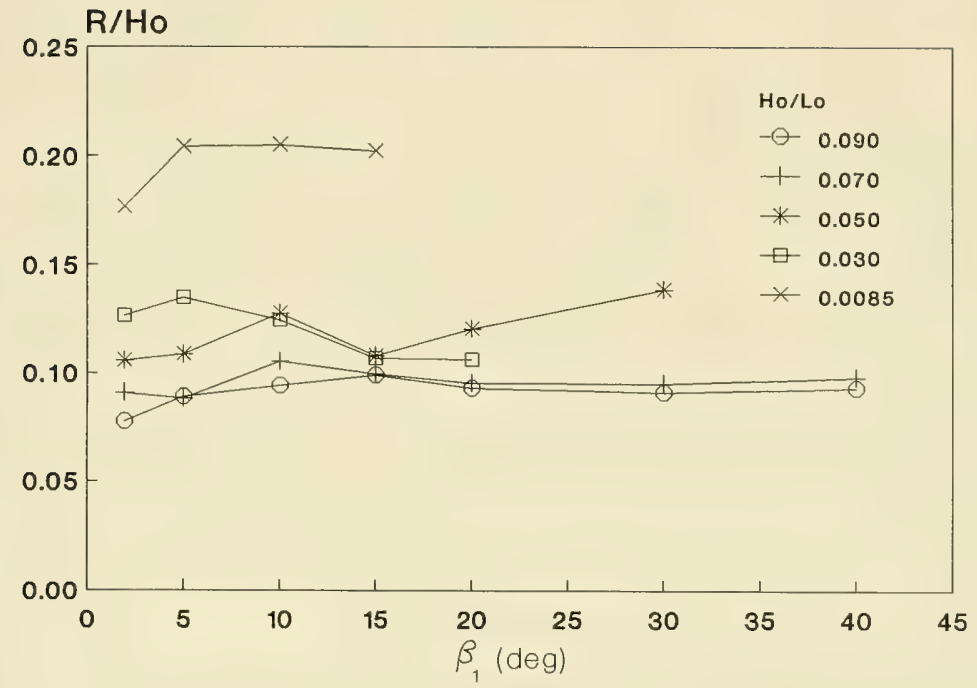

Figure 58. $\mathrm{R} / \mathrm{H}_{\mathrm{o}}$ as a function of $\beta_{1}$

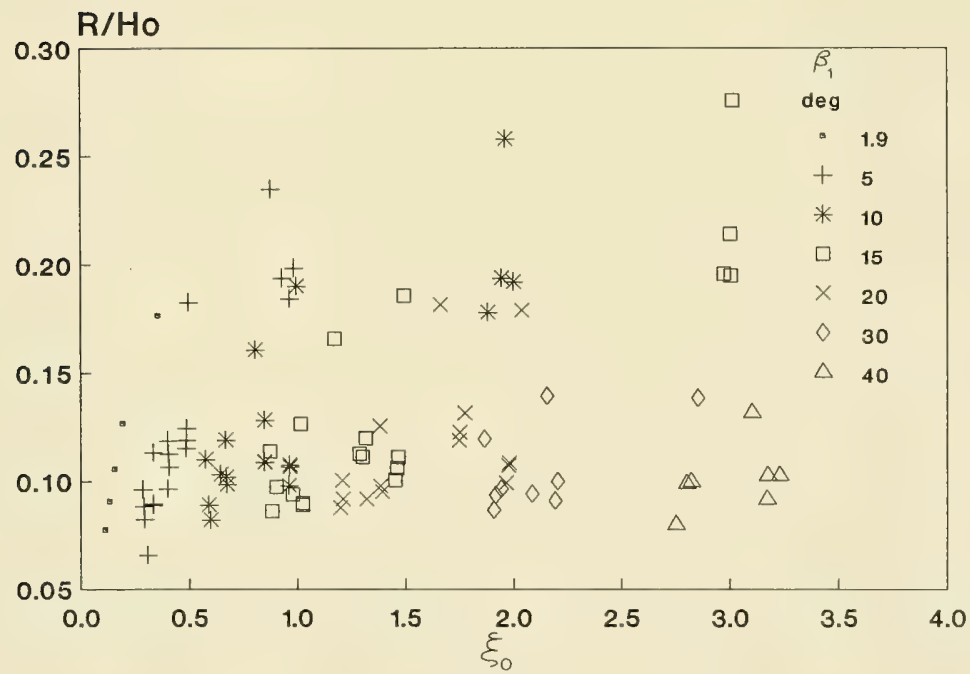

Figure 59. $\mathrm{R} / \mathrm{H}_{\mathrm{o}}$ as a function of $\xi_{0}$ 


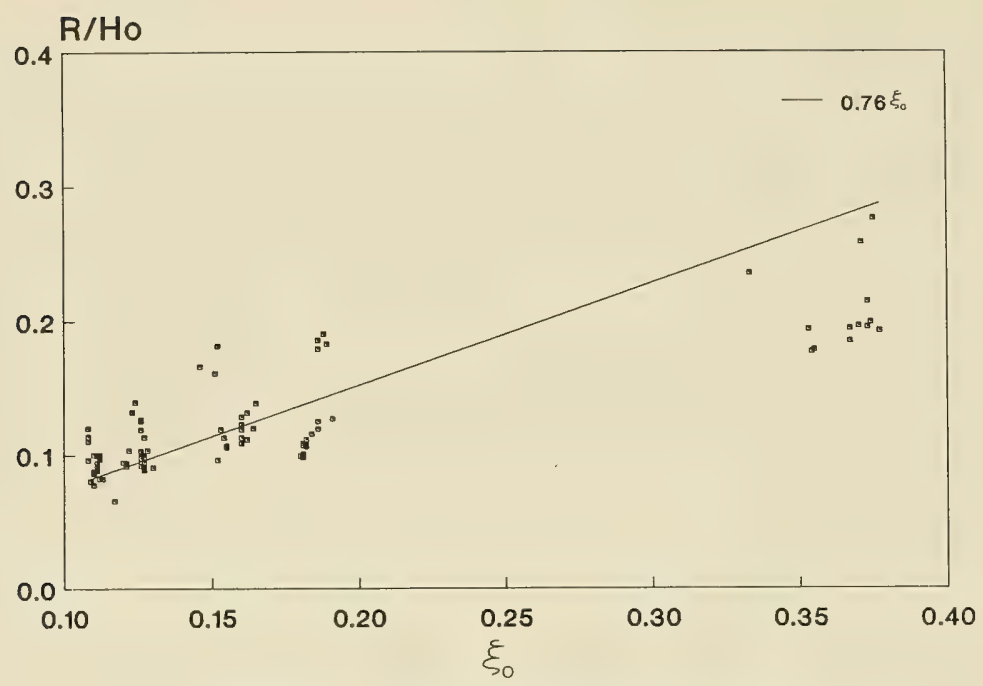

Figure 60. $\mathrm{R} / \mathrm{H}_{\mathrm{o}}$ as a function of $\xi_{0}$ using $m$ as the predominant angle

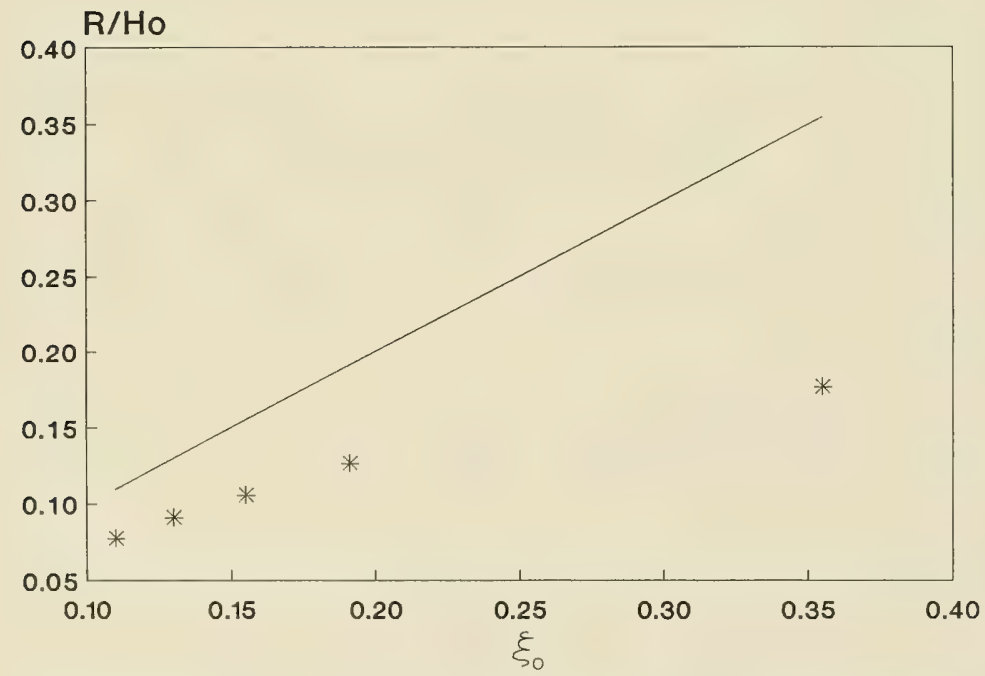

Figure 61. $\mathrm{R} / \mathrm{H}_{\mathrm{o}}$ for $\mathrm{plane-slope}$ cases as a function of $\xi_{0}$ 
155. Holman and Sallenger (1985) analyzed an extensive field data set of runup on a barred beach. Although there was wide scatter in the data, they concluded that runup appeared to depend on $\xi_{0}$. However, the choice of slope with which to calculate $\xi_{0}$ was unclear. The foreslope appeared to be appropriate for data taken at high tide and midtide, whereas the bar slope appeared to "have at least some influence" on setup at low tide. (Runup is defined as the combination of a superelevated MWL, called setup, and a time dependent quantity called swash.) It is not clear if the bar was a major cause of wave breaking in their low-tide measurements. The present tests with regular waves indicate that the bar has a very weak influence, if any, on runup if waves break on the bar. In agreement with Holman and Sallenger, the foreshore, or wide-area slope, appears to be the best quantity to use in correlating $\mathrm{R} / \mathrm{H}_{\circ}$ with $\xi_{0}$; however, only one slope (1/30) was used in the present study, so this conclusion can only be tentative.

\section{Wave Steepness Scaling}

156. A subtest was performed to determine if the magnitude of wave height and period had an effect on wave breaking properties. Cases 6210 , 6220, and 6230 each had a nominal wave steepness $H_{0} / L_{o}=0.05$, but this ratio was obtained with different wave periods and heights, listed in Table 12. Breaker depth and height indices, runup, and reflection coefficients were determined from these tests and are also listed in Table 12. Figures 62(a-c)-64(a-c) show the wave forms at incipient breaking for the base test (a) and the two variations $(b, c)$. The wave forms for the base test and scaled variations show the incipient breaking wave is similar, but not identical, and has the same breaker type for corresponding seaward bar angles. Breaker depth values for the variation cases follow the trend of the base case; however, breaker height indices and reflection coefficients show great variation and no apparent trend. Although runup values show deviations between the variation cases and base cases, the values are nearly constant. Because runup was shown to depend on wave steepness and primary slope (m), not bar slope, this result is not unexpected.

157. The subtest was inconclusive and not successful in proving or disproving if wave period and wave height individually exert influence on wave 


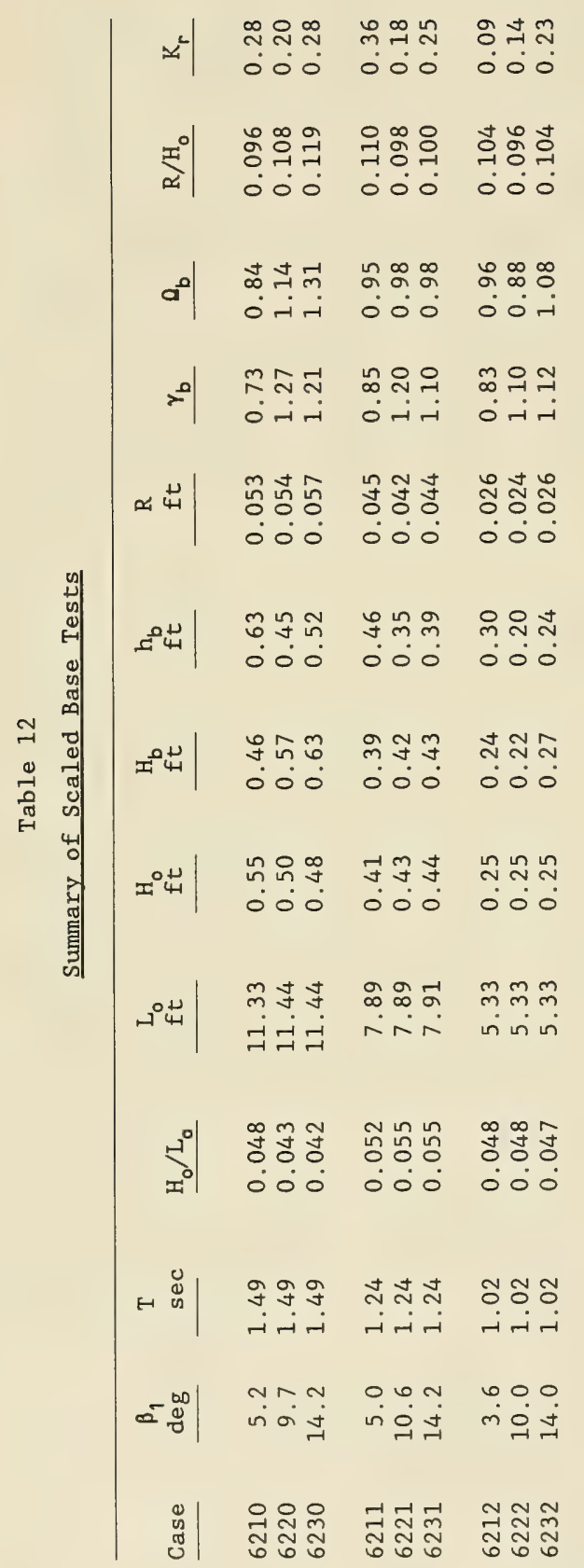



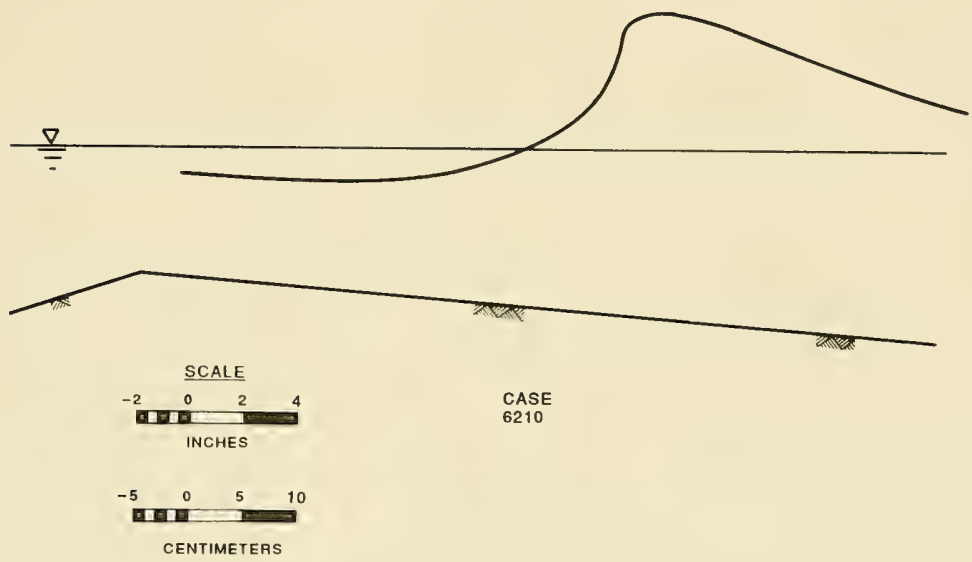

a. Case 6210

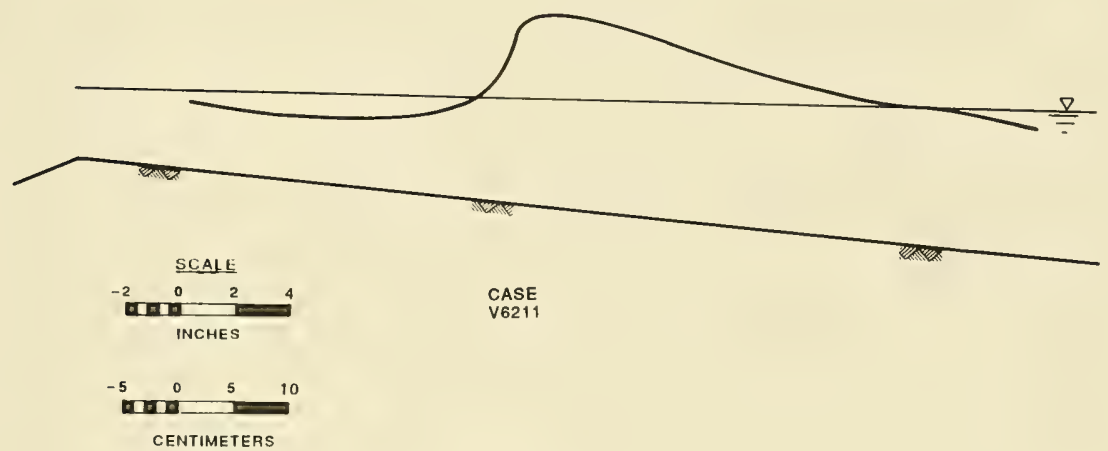

b. Case 6211

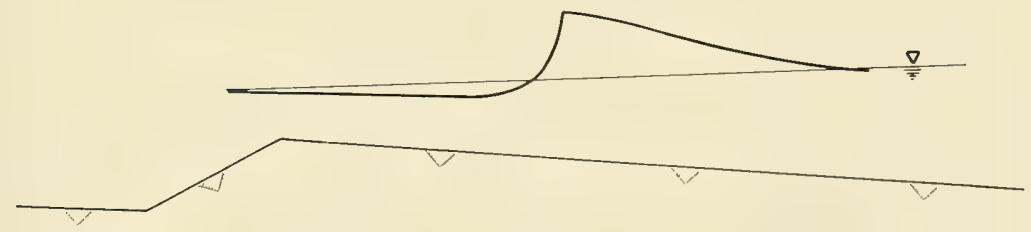

\begin{tabular}{lccccc}
\multicolumn{2}{c}{ SCALE } & \multicolumn{4}{c}{ SCALE } \\
2 & 0 & 2 IN & 5 & 0 & $5 \mathrm{CM}$ \\
& 1 & 1 & & 1 & 1
\end{tabular}

c. Case 6212

Figure 62. Wave at incipient breaking for $\mathrm{H}_{\mathrm{o}} / \mathrm{L}_{\mathrm{o}}=0.05$, $\beta_{1}=5 \mathrm{deg}, \beta_{3}=20 \mathrm{deg}$ 


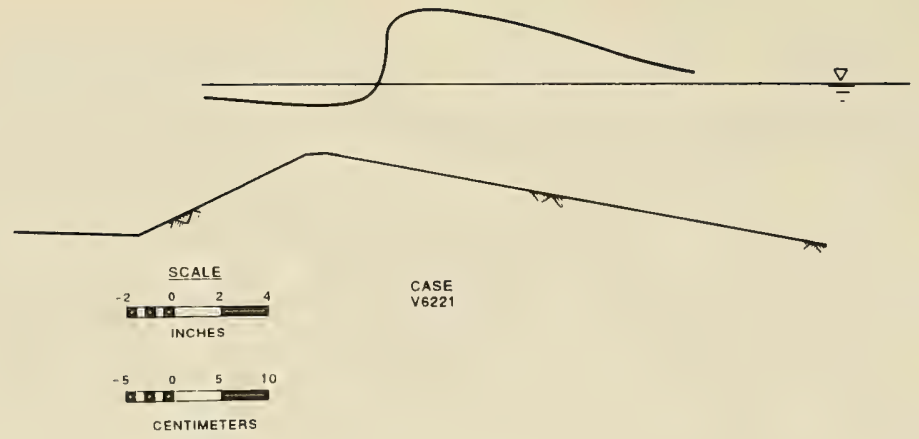

a. Case 6220

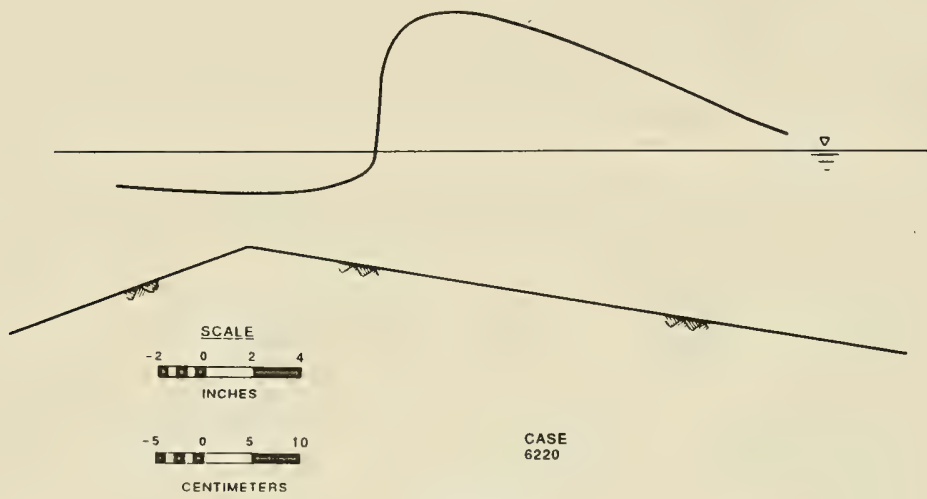

b. Case 6221
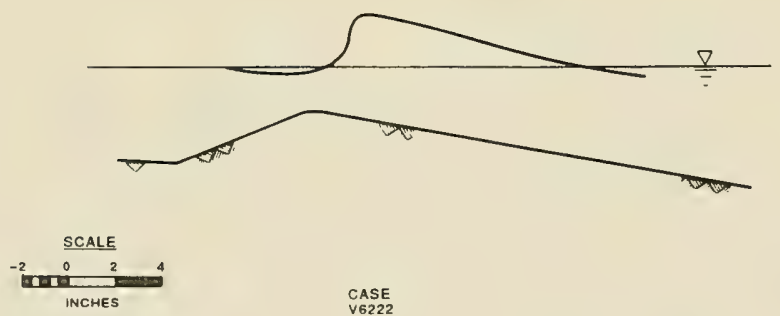

$$
\begin{aligned}
& \text { CASE } \\
& \text { V } 6222
\end{aligned}
$$

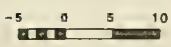

CENTIMETERS

c. Case 6222

Figure 63. Wave at incipient breaking for $\mathrm{H}_{\mathrm{o}} / \mathrm{L}_{\mathrm{o}}=0.05$, $\beta_{1}=10 \mathrm{deg}, \beta_{3}=20 \mathrm{deg}$ 


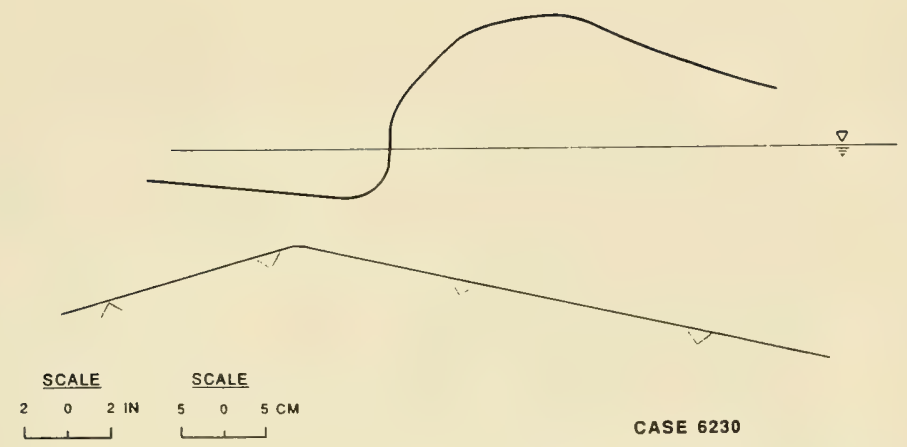

a. Case 6230

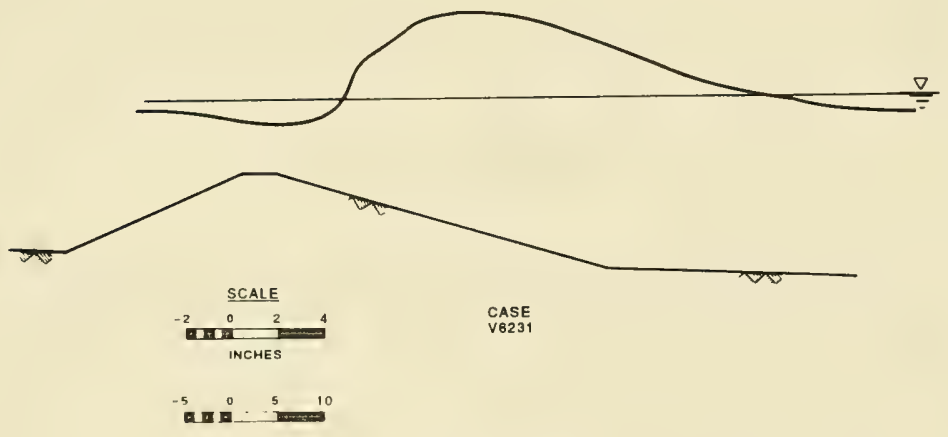

b. Case 6231

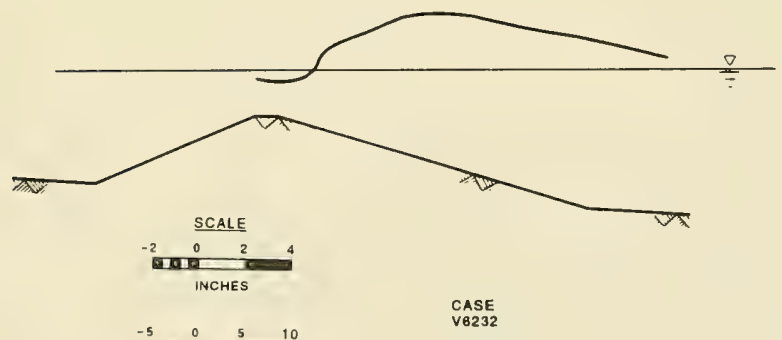

- 2010

CENTIMETERS

c. Case 6232

Figure 64. Wave at incipient breaking for $H_{0} / L_{0}=0.05$, $\beta_{1}=15 \mathrm{deg}, \beta_{3}=20 \mathrm{deg}$ 
breaking properties for the same wave steepness. Reasons the subtest were indeterminate are:

a. Deviations of the phenomenon were within the range of deviation for the experiment.

b. The range of the variables that determined deepwater wave steepness, $H_{0}$ and $L_{o}$, was only a factor of two.

c. The independent variables, such as $\beta_{1}$ and $H_{0} / L_{0}$, changed slightly between the base case and variation cases because of limitations in equipment.

d. Accuracy of the data decreased as the wave height decreased because of limitations in measurements.

A complicating factor is the wide variability in reflection, which is not known. The validity of steepness scaling should be pursued in future work, and it is recommended that the various wave conditions be generated on a plane slope prior to installing bars to establish a control condition by eliminating bar variables that are difficult to specify with precision.

\section{Irregular Waves}

158. Irregular waves were generated, recorded, and analyzed for 500 waves, contrary to the monochromatic wave tests that were analyzed for 15 waves. Because wave height and period varied, a longer record was required for the irregular wave tests to obtain a statistically strong confidence interval for calculating the wave spectrum. Reflection and re-reflection between the beach and the wave board could not be avoided in the irregular wave tests.

159. Maximum, significant, and rms wave height were calculated from the time series of the irregular wave trains and are plotted versus distance from the still-water shoreline in Figures $65(\mathrm{a}-\mathrm{c})-68(\mathrm{a}-\mathrm{c})$. Figures $65(\mathrm{a}-\mathrm{c})-68(\mathrm{a}-\mathrm{c})$ show that the difference between $H_{\max }, H_{s}$, and $H_{r m s}$ decreases as the waves enter shallow water, which indicates the distribution of wave heights becomes narrower, i.e., the waves become more "regular." This behavior was also shown in other irregular wave laboratory and field studies, such as Thompson and Vincent (1984) and Ebersole and Hughes (1987).

160. Wave height decay for the plane-beach tests is extremely gentle, making it difficult to define a mean breaker line as discussed by Thornton, Wu, and Guza (1985). The barred tests depict a decrease in wave height immediately shoreward of the bar, but the wave height over the bar (Gage 4) was not 


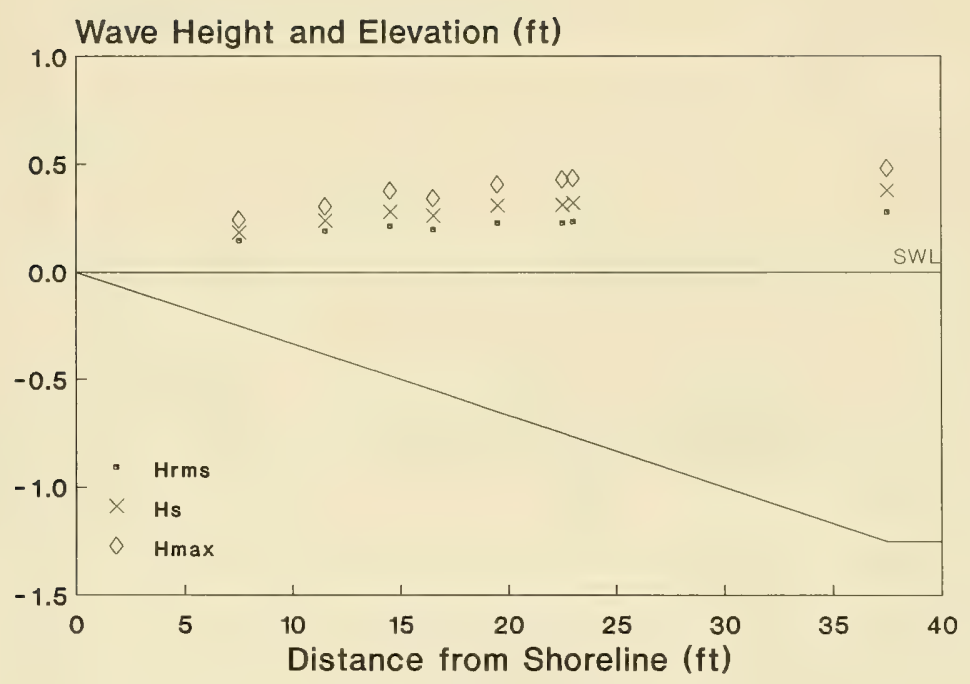

a. Case R2000

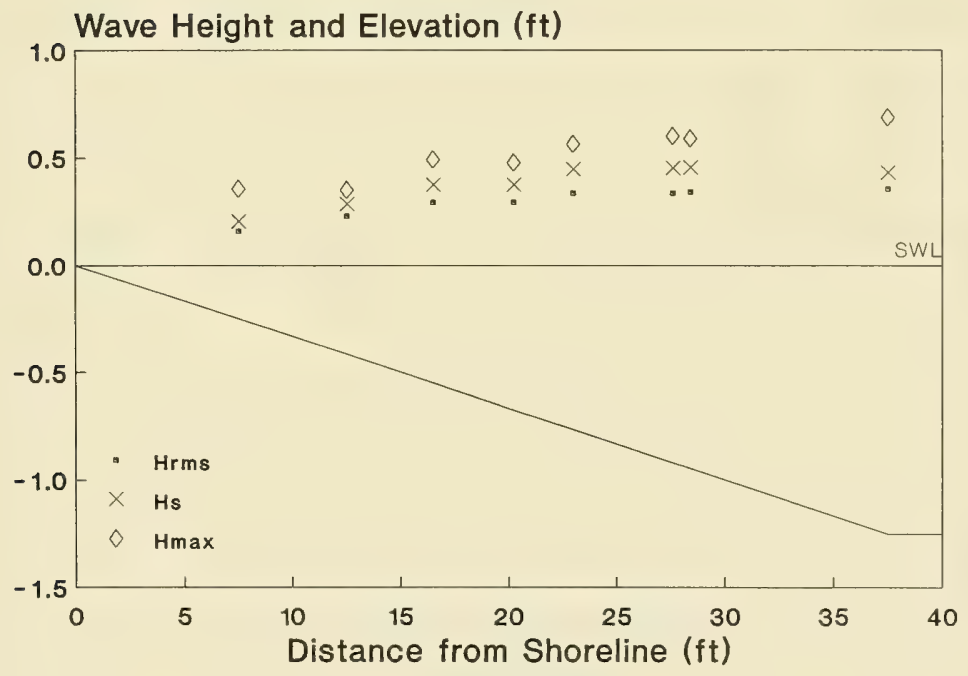

b. Case R6000

Figure 65. $\mathrm{H}_{\max }, \mathrm{H}_{\mathrm{s}}$, and $\mathrm{H}_{\mathrm{rms}}$ as a function of distance, $\mathrm{m}=1 / 30$ (Continued) 


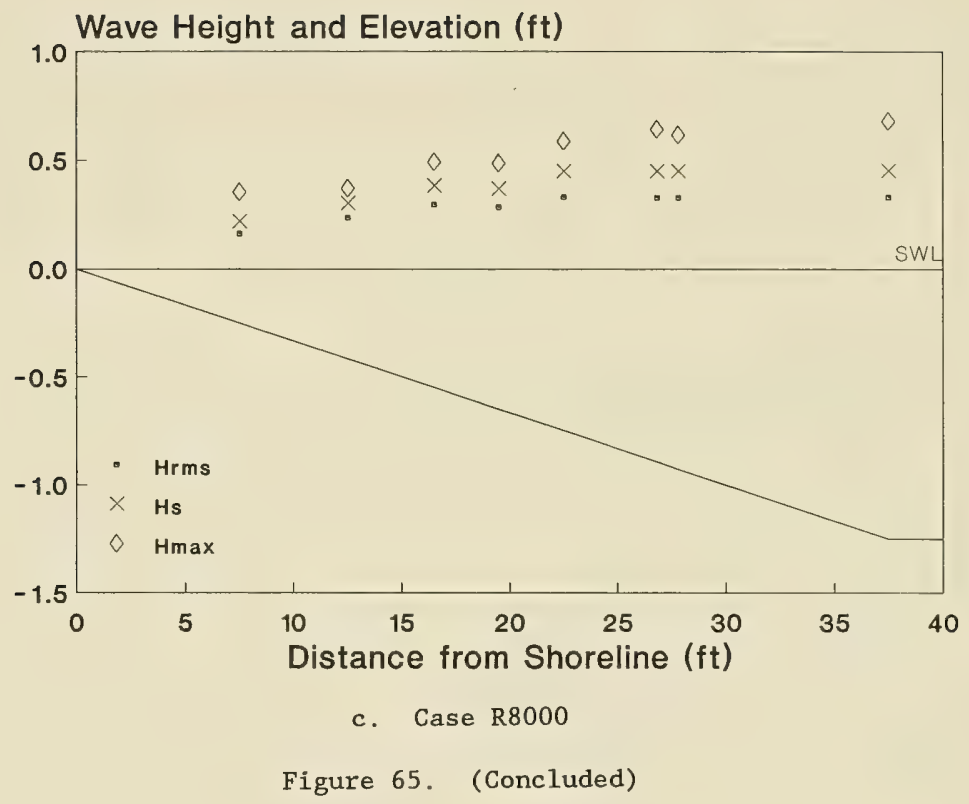




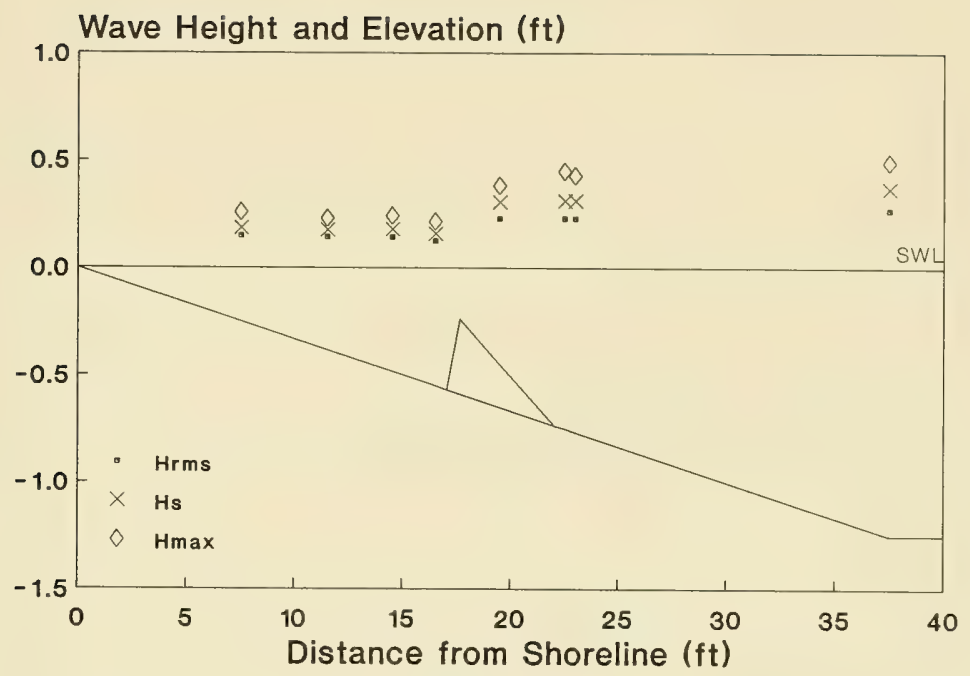

a. Case R2210

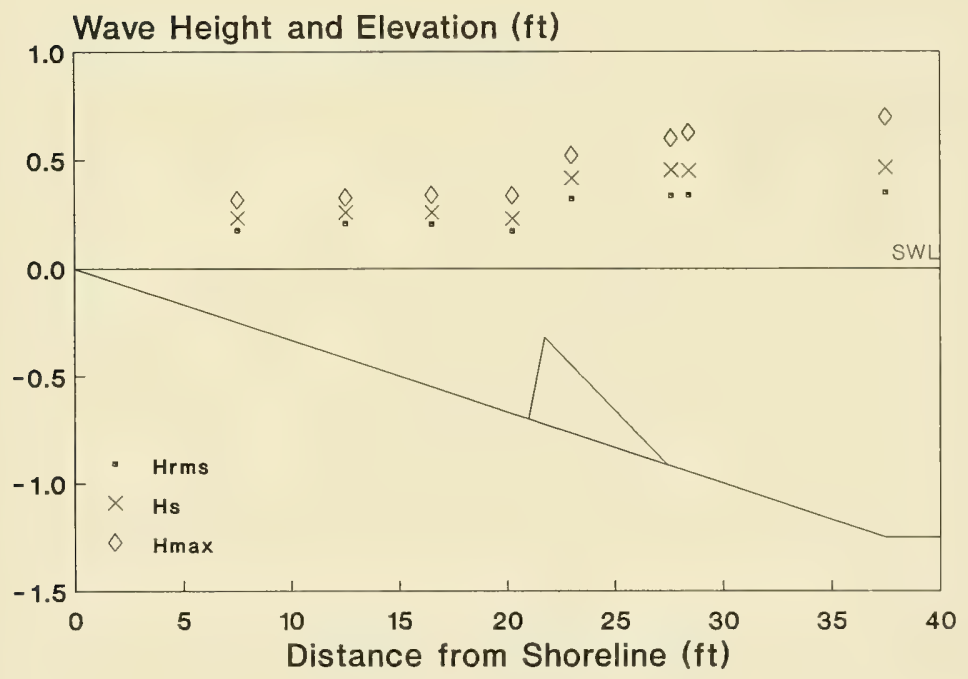

b. Case R6210

Figure 66. $H_{\max }, \mathrm{H}_{\mathrm{s}}$, and $\mathrm{H}_{\mathrm{rms}}$ as a function of distance, $\beta_{1}=5 \mathrm{deg}$ (Continued) 


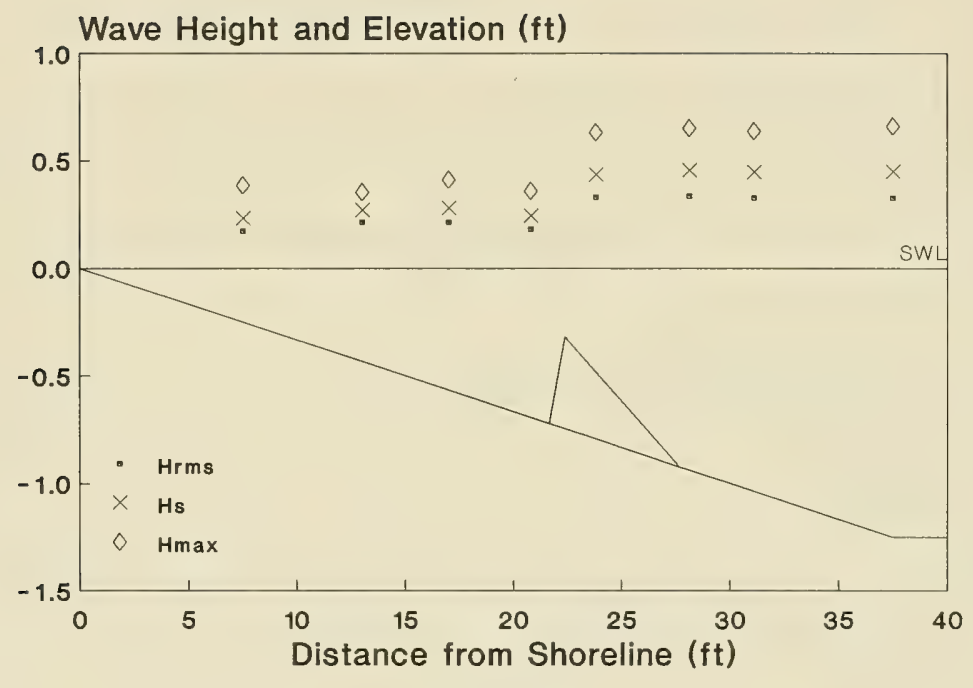

c. Case R8210

Figure 66. (Concluded) 


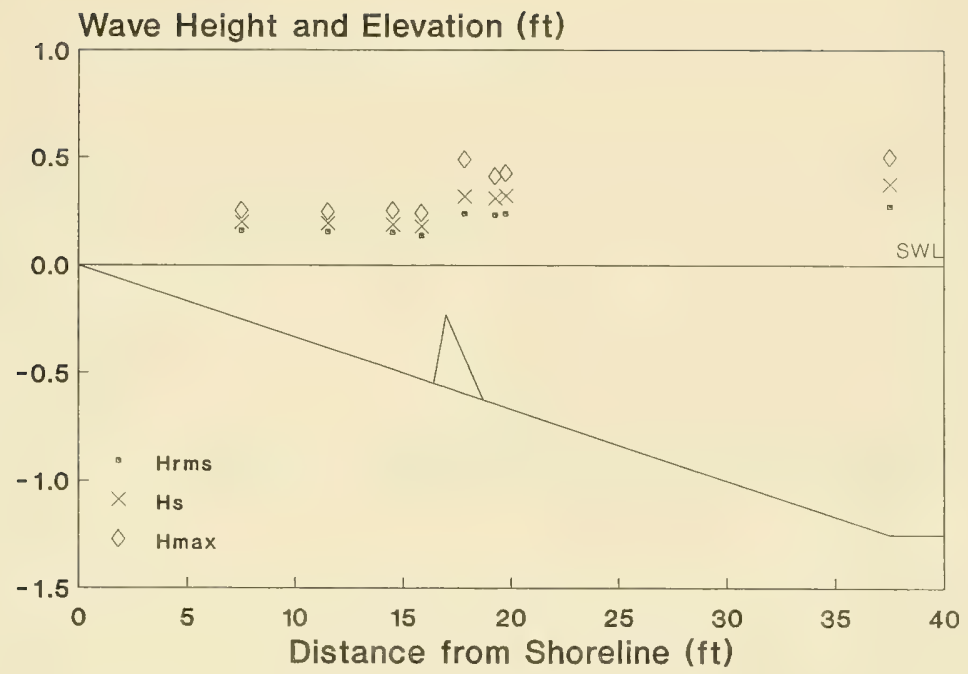

a. Case R2220

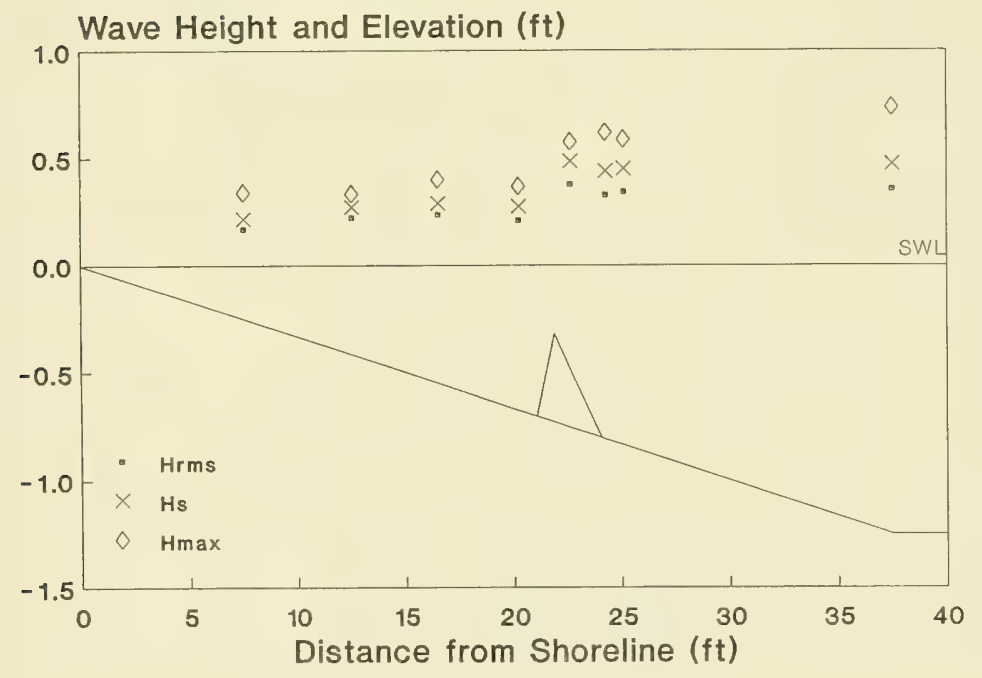

b. Case R6220

Figure 67. $H_{\max }, H_{s}$, and $H_{\text {rms }}$ as a function of distance, $\beta_{1}=10 \mathrm{deg}$ (Continued) 


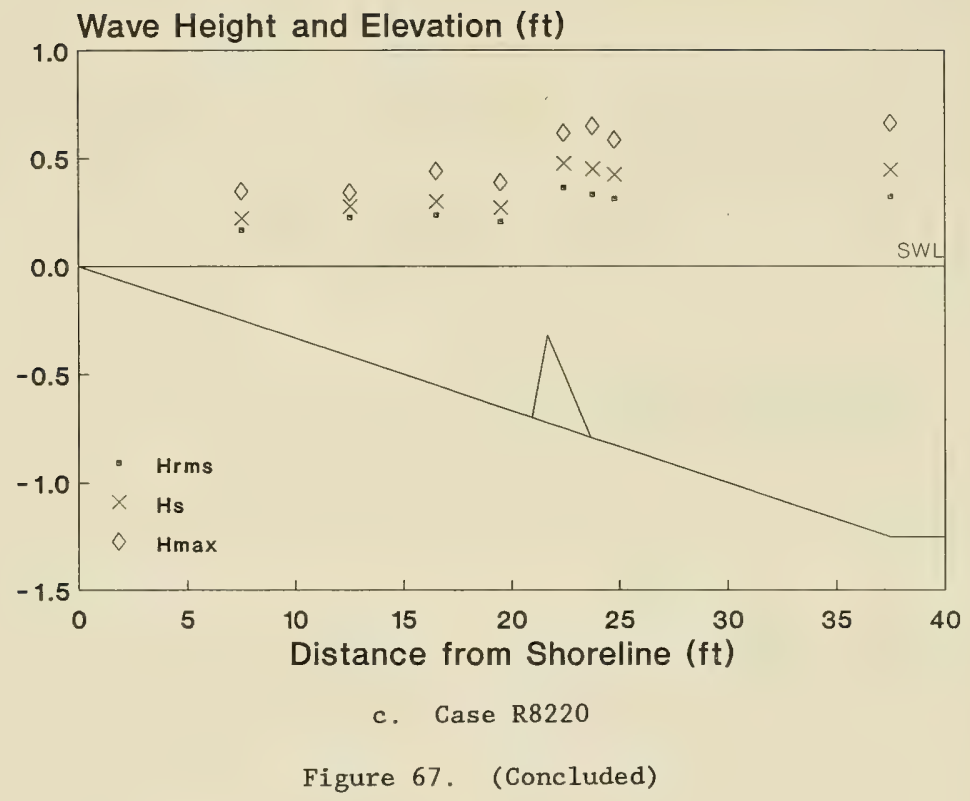




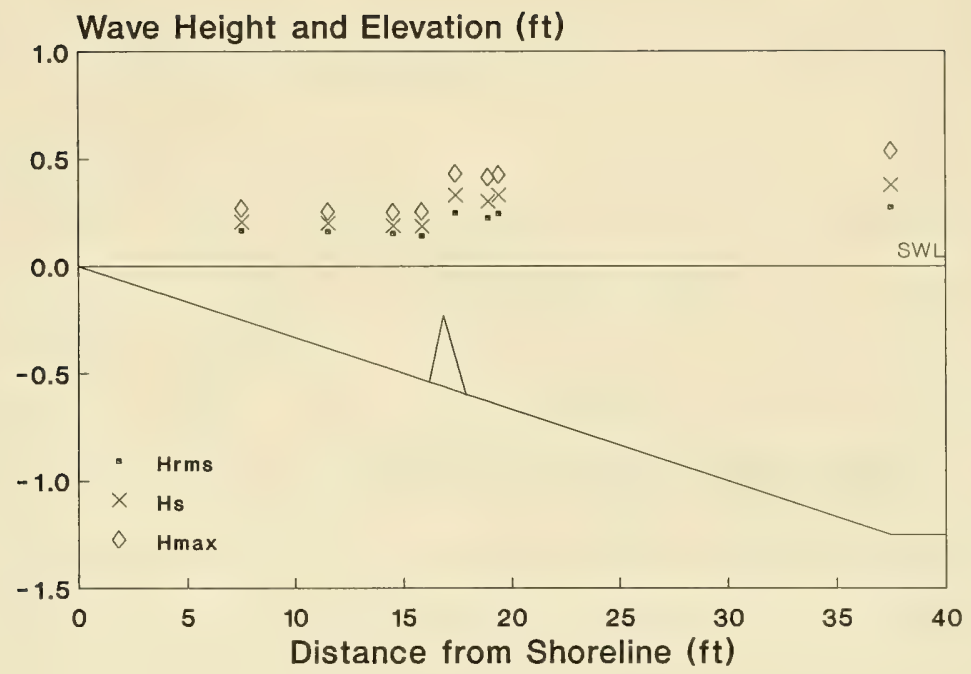

a. Case R2230

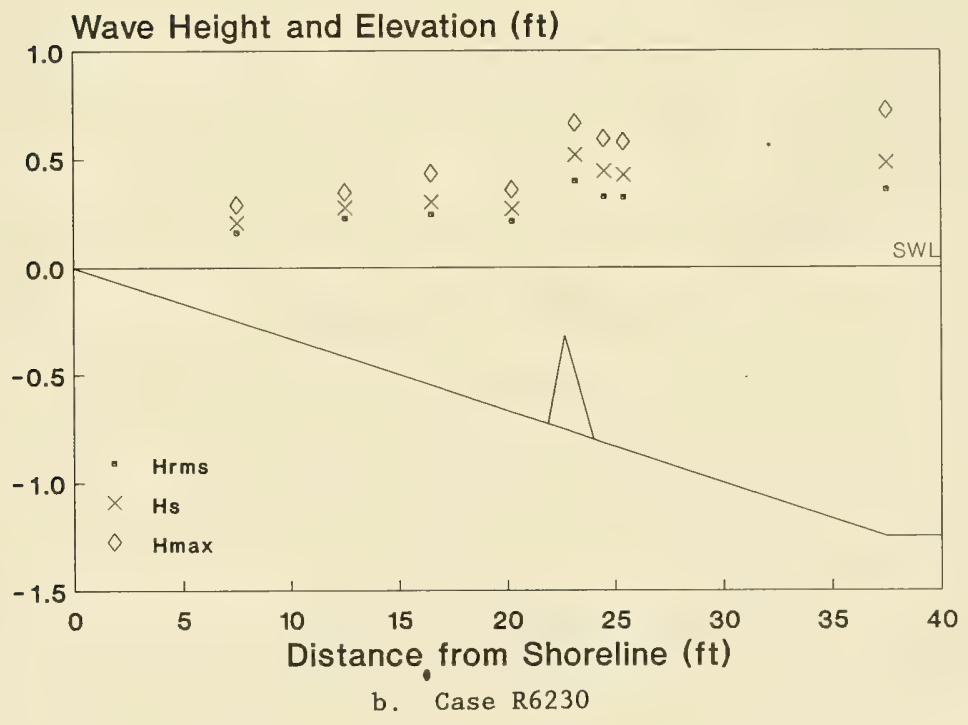

Figure 68. $\mathrm{H}_{\max }, \mathrm{H}_{\mathrm{s}}$, and $\mathrm{H}_{\mathrm{rms}}$ as a function of distance, $\beta_{1}=15 \mathrm{deg}$ (Continued) 


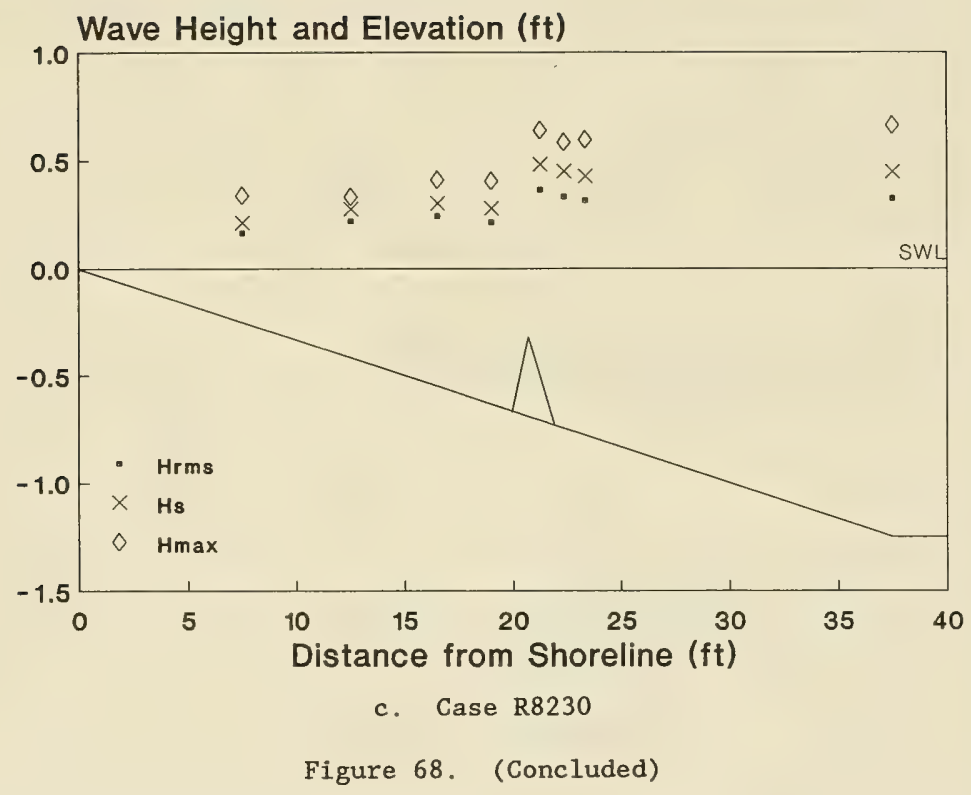


always the maximum in the shoaling transformation. Because a steep decay in wave height occurred between Gages 4 and 5 for tests involving a bar, the height at Gage 4 was used as the breaking wave height in analysis of both the barred and the plane-sloped cases.

161. Statistical wave heights derived from data from Gage 4 were compared with Equation 34, the breaker height expression for irregular waves (Goda 1975). Goda assumed the breaker height would vary and gave a range to the coefficient $A$ to predict this variation. In the present study, it seemed reasonable to compare the higher value of $A$ with the maximum wave height measured at Gage 4. Figure 69 shows the predicted wave height given by Equation 34 , in which the coefficient $A$ was the maximum specified by Goda plotted versus $H_{\max }$ normalized by $\left(\mathrm{H}_{\mathrm{s}}\right)_{0}$. The line of perfect prediction is also plotted in Figure 69 . The Goda equation predicts the lower measured values well, but for tests with steep-faced bars, resulting in higher $\xi_{0}$-values, the equation gives an overprediction.

162. Because Equation 34 (Goda 1975) predicted $H_{\max }$ well using the upper limit of A, the lower limit of A was used to estimate a statistically averaged wave height. Goda arbitrarily selected the lower limit to be two-thirds of the upper limit; therefore, significant wave height was used since it is the average of the highest one-third wave heights. Predicted values of $\left(\Omega_{b}\right)_{s}$ using $A=0.12$ in Equation 34 were plotted against the significant wave height normalized by $\left(\mathrm{H}_{\mathrm{s}}\right)_{0}$ at Gage 4 in Figure 70 . The predicted values of $\left(\Omega_{\mathrm{b}}\right)_{\mathrm{s}}$ agree well with the measurements for $\beta_{1} \leq 10 \mathrm{deg}$; but are too high for $\beta_{1}=15 \mathrm{deg}$.

163. With the reasonable success in associating A-values with $H_{\max }$ and $H_{s}$, a procedure was developed to determine the A-value required to predict $\mathrm{H}_{\mathrm{rms}} /\left(\mathrm{H}_{\mathrm{s}}\right)$ 。. Because the Rayleigh distribution gives $\mathrm{H}_{\mathrm{s}}=\sqrt{2} \mathrm{H}_{\mathrm{rms}}$, the minimum value of the empirical coefficient recommended by Goda (1975) was reduced by $\sqrt{2}$, which yielded $A=0.085$. Predictions with this value of $A$ gave reasonable results for all seaward angles, but it underestimated the measured values for $\beta_{1} \leq 5 \mathrm{deg}$. Therefore, the coefficient was rounded to $A=0.09$. The predicted versus measured values using $A=0.09$ are shown in Figure 71 .

164. Equation 34 predicts the measured wave heights well for the planeslope cases, and for the barred slope cases for $\beta_{1} \leq 10 \mathrm{deg}$. The equation overpredicted wave heights in the tests with the steeper bar angle 


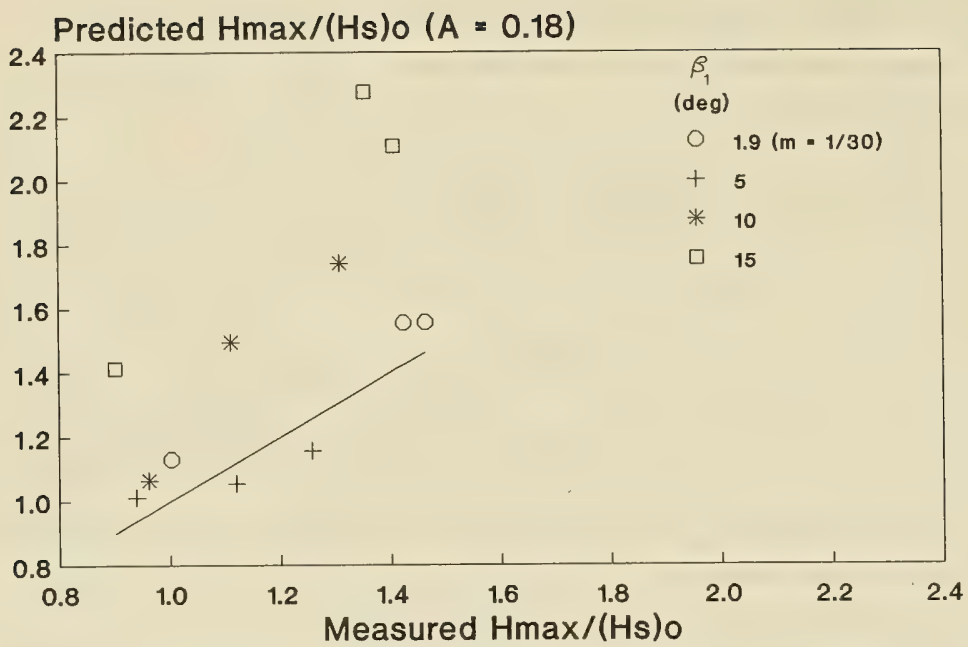

Figure 69. Predicted $\mathrm{H}_{\max } /\left(\mathrm{H}_{\mathrm{s}}\right)_{0}$ of Goda (1975)

$(A=0.18)$ as a function of measured $H_{\max } /\left(H_{s}\right)$ 。

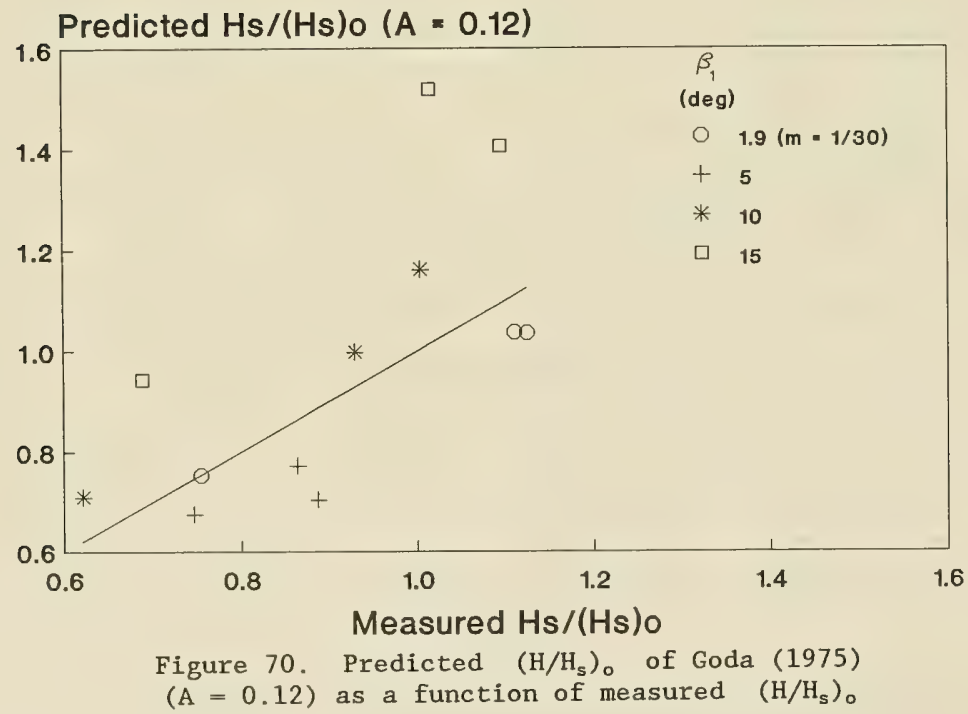




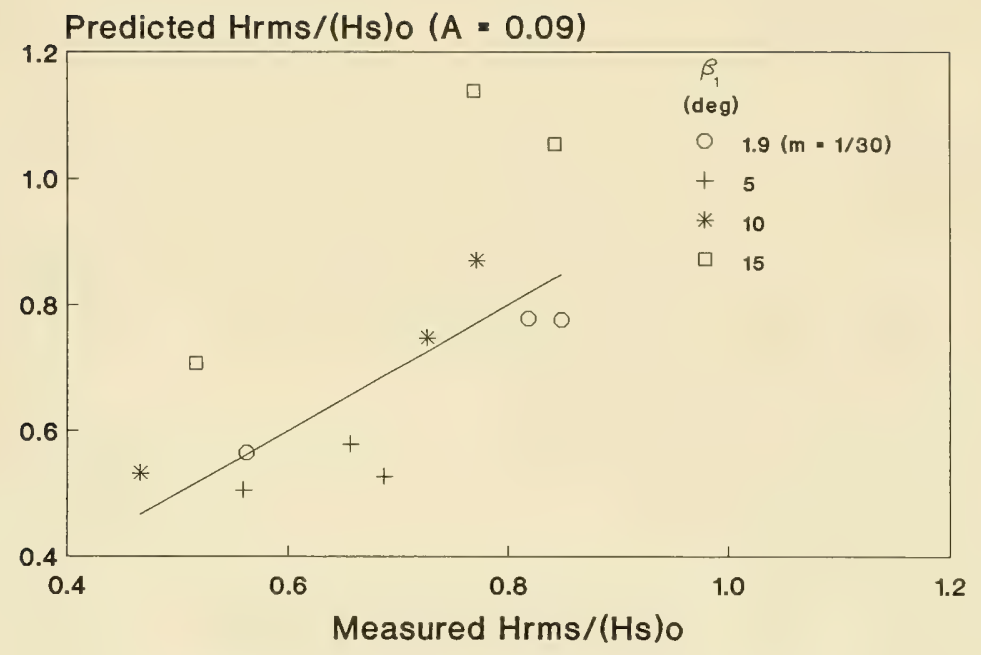

Figure 71. Predicted $\mathrm{H}_{\mathrm{rms}} /\left(\mathrm{H}_{\mathrm{s}}\right)$ 。 of Goda (1975) $(A=0.09)$ as a function of measured $H_{r m s} /\left(H_{s}\right)$ 。

$\left(\beta_{1}=15 \mathrm{deg}\right)$. Other procedures are clearly required to predict heights over engineering structures having steep faces.

165. Figures $72(\mathrm{a}-\mathrm{c})$ to $75(\mathrm{a}-\mathrm{c})$ show $\mathrm{H}_{\mathrm{rms}} / \mathrm{h}$ as a function of distance from the shoreline, where $h$ is the depth evaluated from SwL. The tests conducted with bars show a significant increase of $H_{r m s} / h$ over the bar. The increase results from the water depth becoming shallow and waves becoming higher by shoaling and becoming nonlinear in shape over the bar. Wave height to water depth decreases directly shoreward of the bar because water depth is deeper and a majority of the waves broke on the bar. The ratio continues to increase as water depth decreases in the surf zone for all tests, including tests on the plane slope. The plots indicate that wave height does not decay consistently with the decrease in water depth; therefore, $\mathrm{H}_{\mathrm{rms}} / \mathrm{h}$ is not constant through the surf zone for either barred profiles or plane-sloping beaches.

166. On the basis of their field measurements, Sallenger and Howd (1989) found $\mathrm{H}_{\mathrm{rms}} / \mathrm{h}$ to be constant, independent of offshore wave conditions, and stated that the wave distribution was energy saturated through the inner surf zone. They concluded that offshore migration of nearshore bars is, therefore, not necessarily associated with the break-point processes; however, 


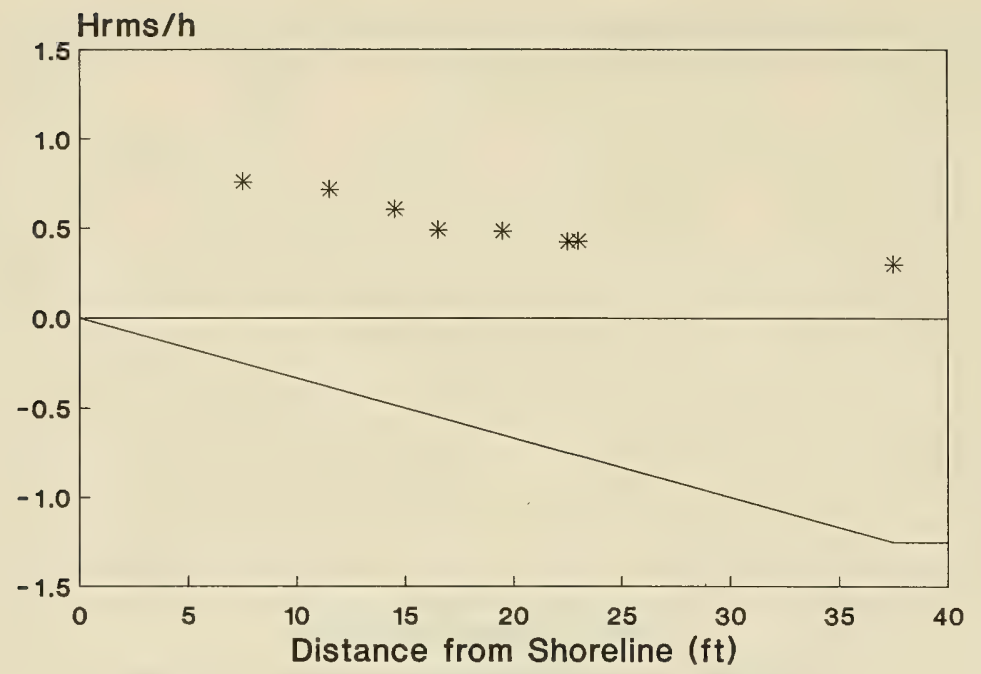

a. Case R2000

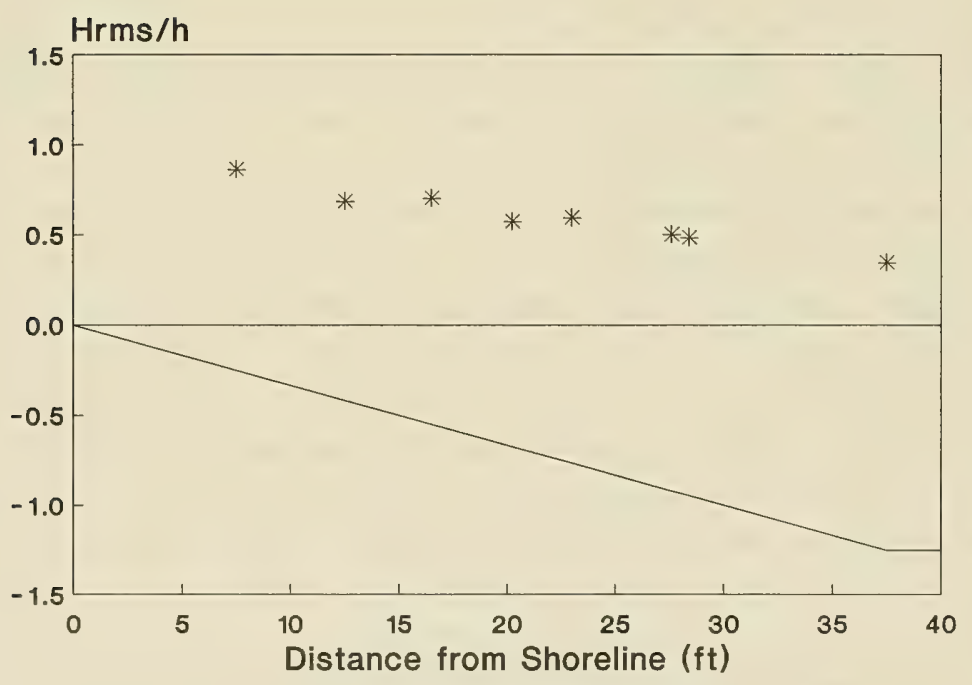

b. Case R6000

Figure 72. $\mathrm{H}_{\mathrm{rms}} / \mathrm{h}$ as a function of distance, $m=1 / 30$ (Continued) 


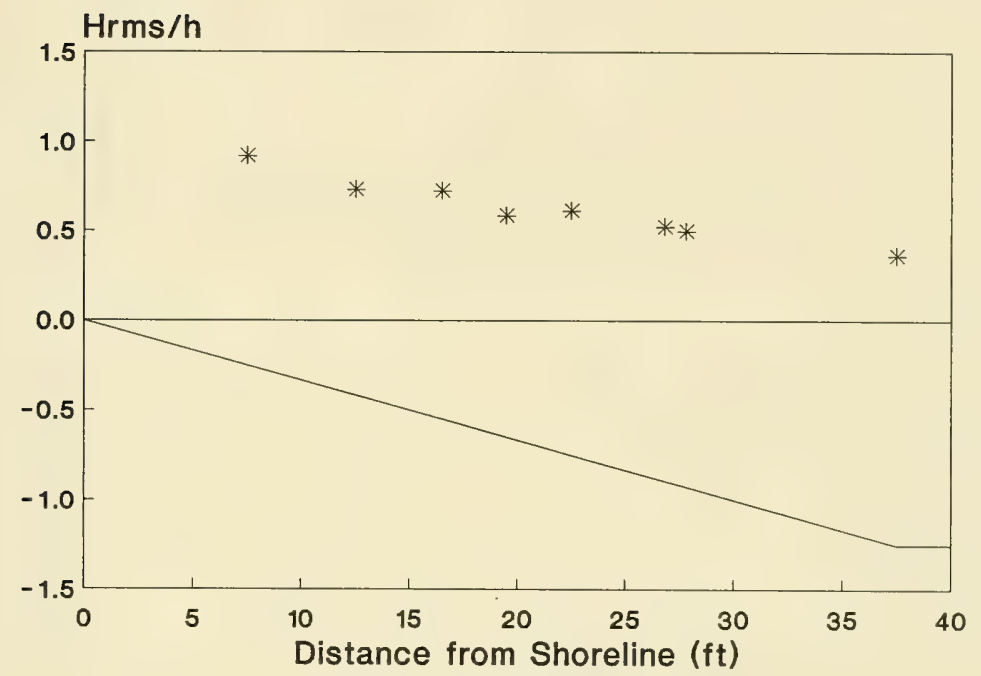

c. Case R8000

Figure 72. (Concluded) 


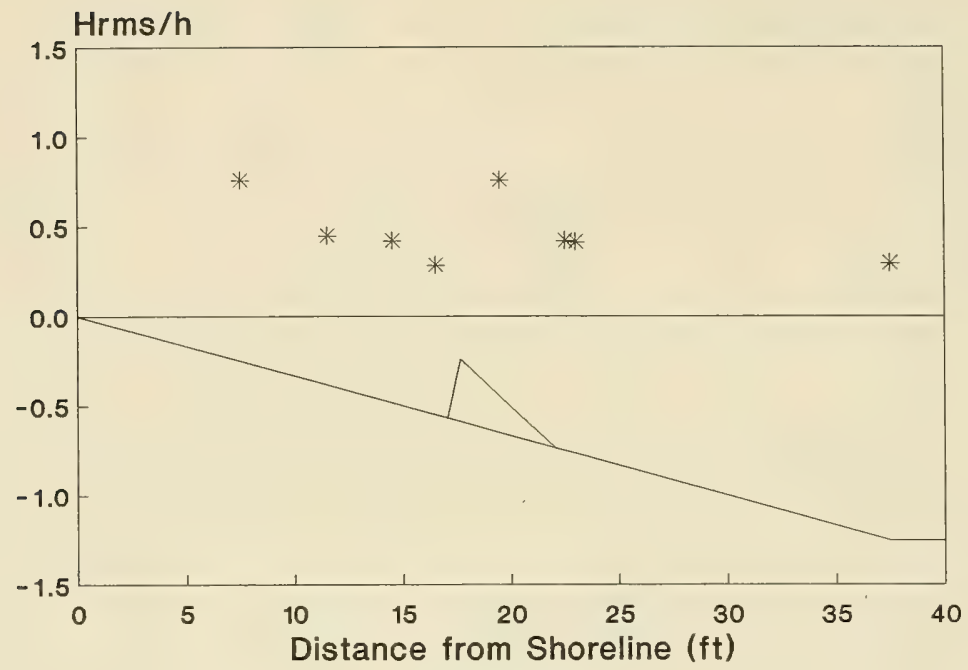

a. Case R2210

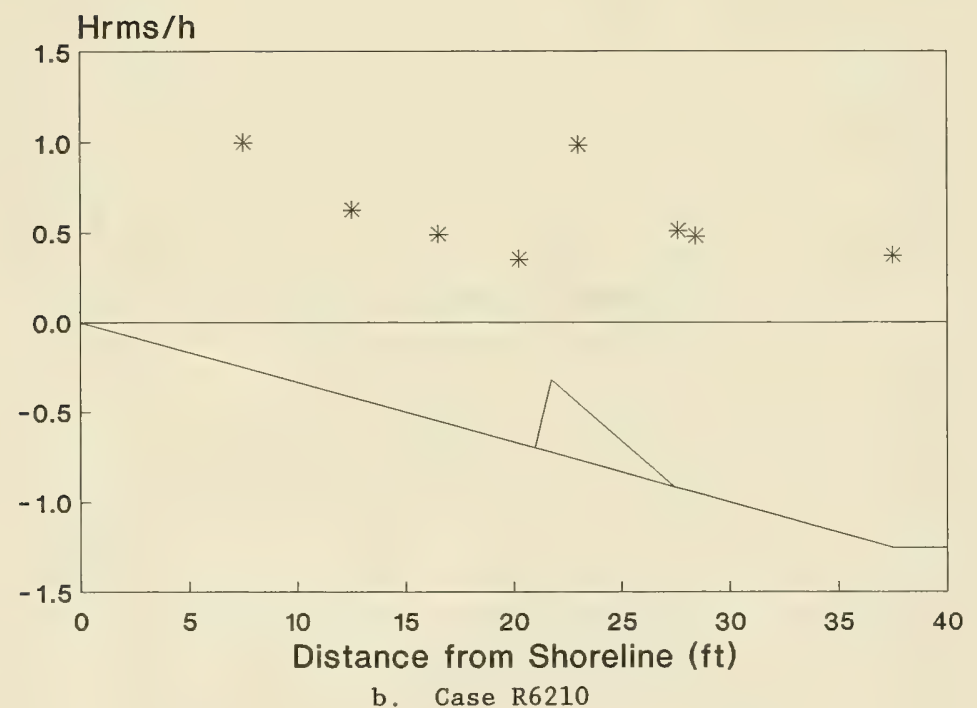

Figure 73. $\mathrm{H}_{\mathrm{rms}} / \mathrm{h}$ as a function of distance, $\beta_{1}=5 \mathrm{deg}$ (Continued) 


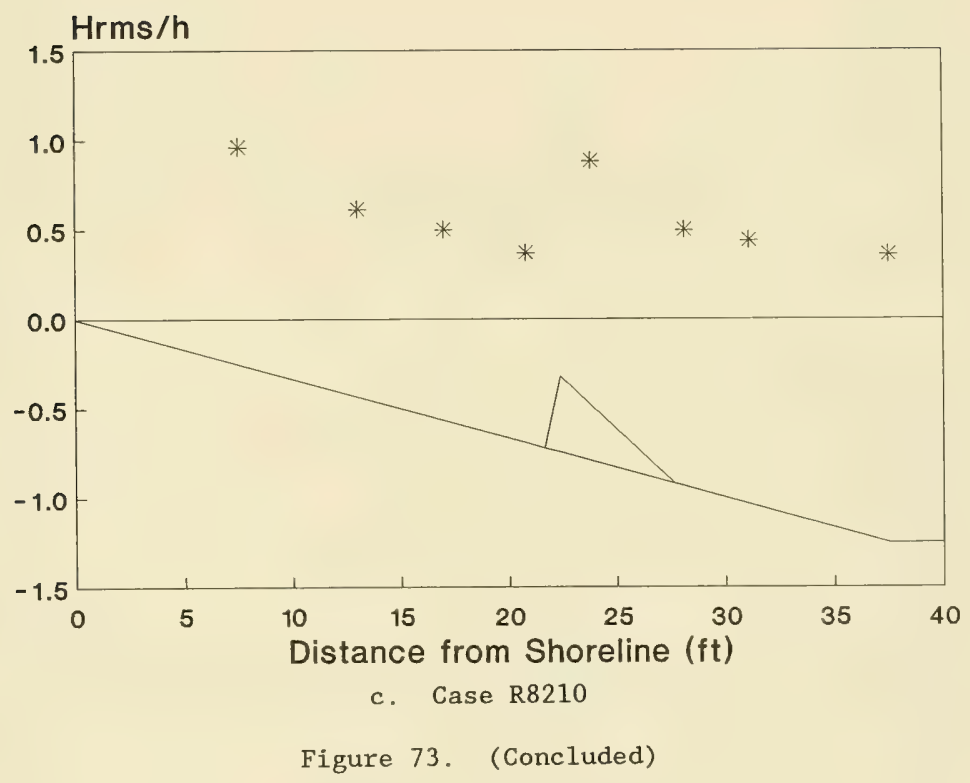




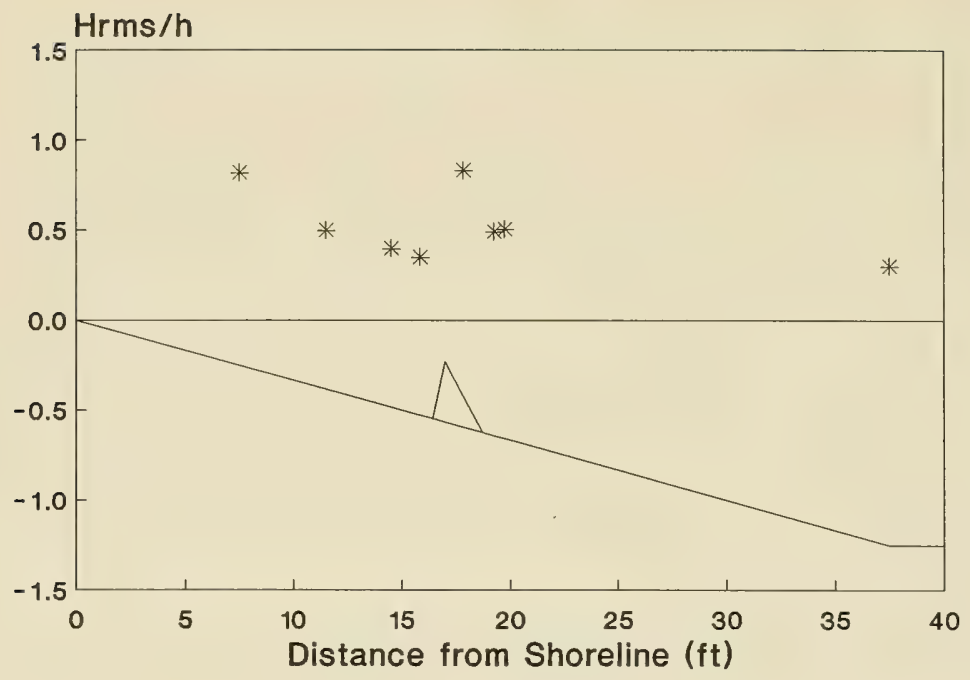

a. Case R2220

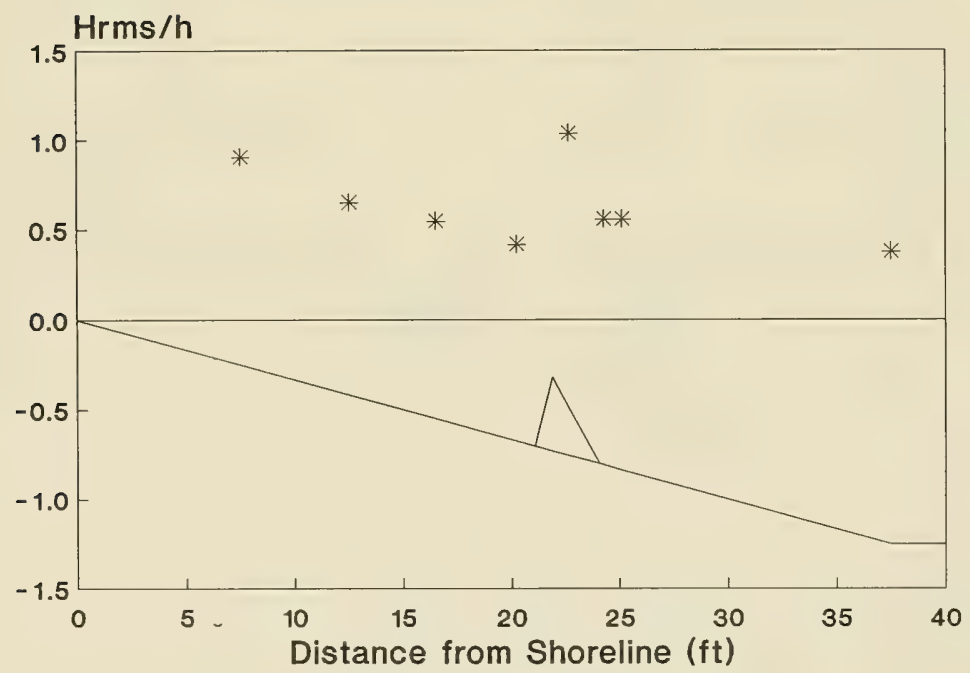

b. Case R6220

Figure 74. $\mathrm{H}_{\mathrm{rms}} / \mathrm{h}$ as a function of distance, $\beta_{1}=10 \mathrm{deg}$ (Continued) 


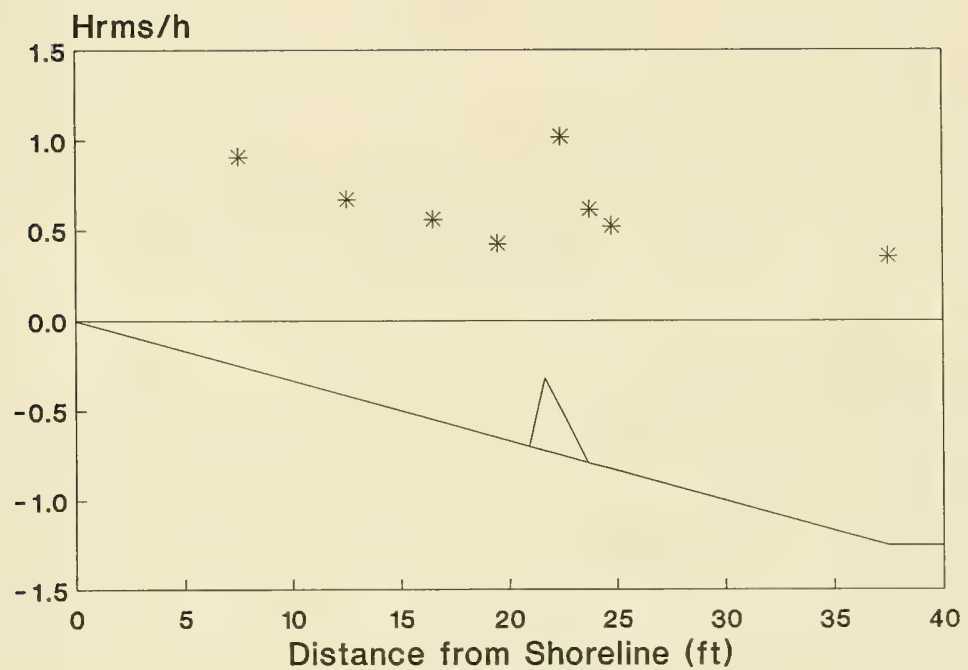

c. Case R8220

Figure 74. (Concluded) 


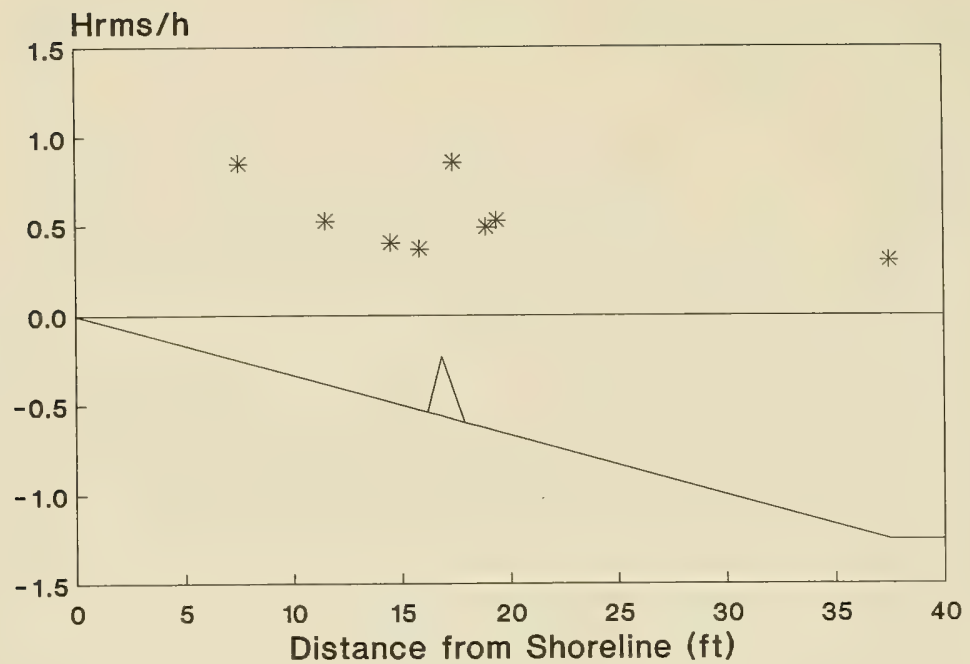

a. Case R2230

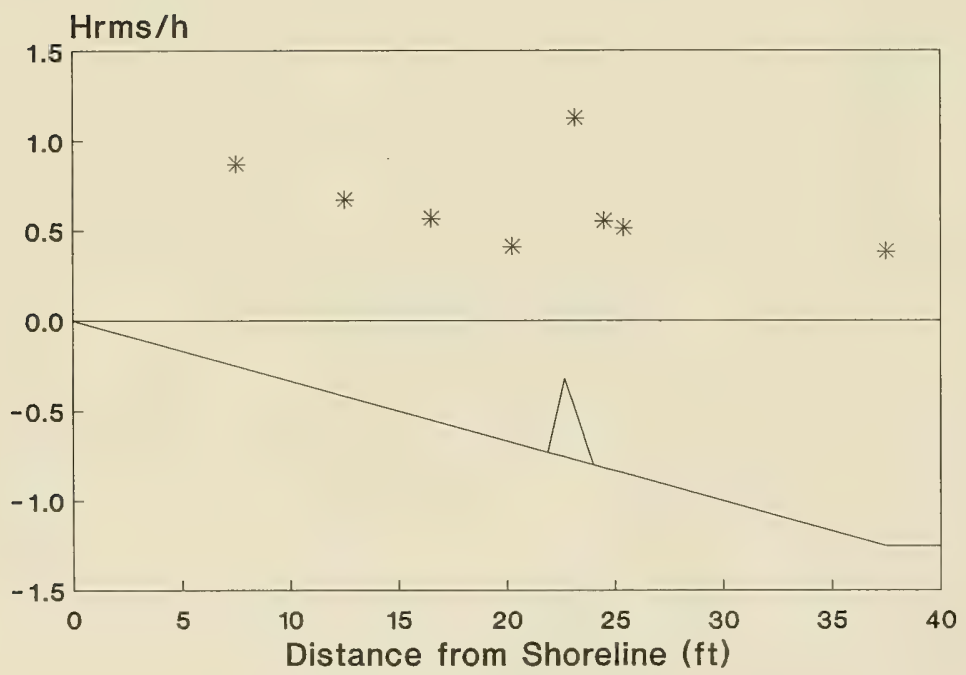

b. Case R6230

Figure 75. $\mathrm{H}_{\mathrm{rms}} / \mathrm{h}$ as a function of distance, $\beta_{1}=15 \mathrm{deg}$ (Continued) 


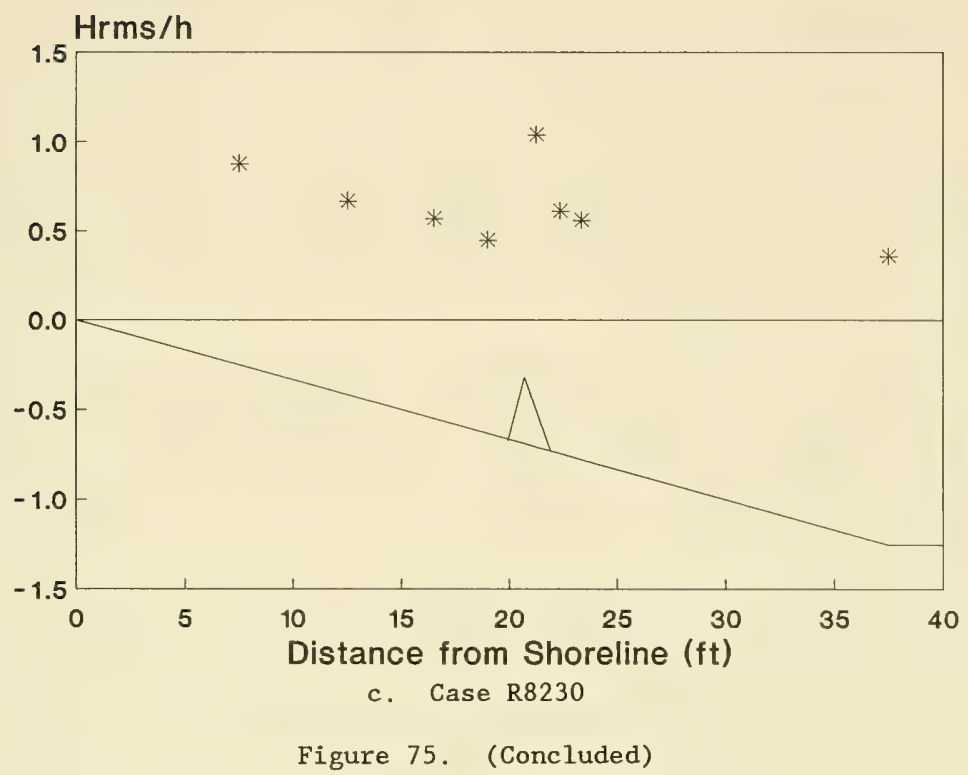

the results of the present study bring into question their conclusion that $\mathrm{H}_{\mathrm{rms}} / \mathrm{h}$ is constant through the surf zone on a barred profile. Sallenger and Howd measured wave heights seaward of an inner bar, and Figures $72(a-c)$ to $75(a-c)$ show $H_{r m s} / h$-values are uniform seaward of the bar. Because Sallenger and Howd apparently did not measure wave height shoreward of the inner bar, they did not find a varying $\mathrm{H}_{\mathrm{rms}} / \mathrm{h}$.

167. Runup measurements for irregular waves were taken for 13 to 34 waves near the start of the test, prior to estimated re-reflection from the wave board; therefore, the irregular wave runup data are not statistically reliable. Average wave runup normalized by $\left(\mathrm{H}_{\mathrm{s}}\right)_{0}$ is plotted as a function of $\xi_{0}$ using the seaward angle $\beta_{1}$ in Figure 76 . The predicted values by Equation 55 (Ahrens 1981) are shown as the solid line. Equation 55 estimates runup well for the plane-slope cases, but underpredicts the measurements for tests with barred profiles. Runup normalized by $\left(H_{s}\right)_{0}$ is independent of $\xi_{0}$ for the barred profiles. Runup was plotted as a function of $\xi_{0}$ with the $1 / 30$ slope used as the primary angle (Figure 77). Equation 55 gives good results if the $1 / 30$ slope is used in the surf similarity parameter. 


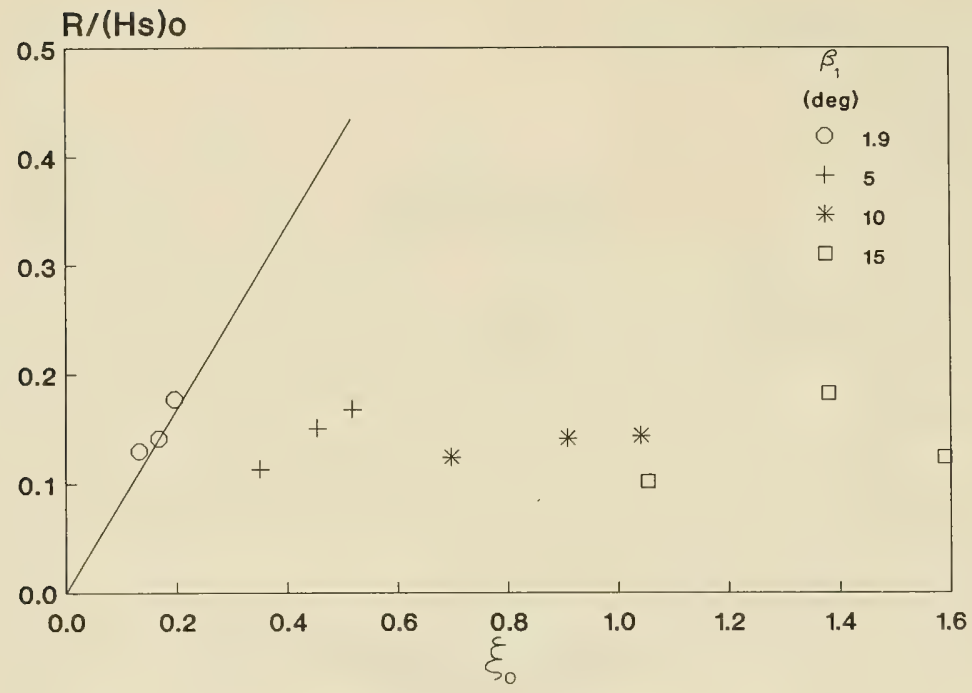

Figure 76. $\mathrm{R} /\left(\mathrm{H}_{\mathrm{o}}\right)_{\mathrm{s}}$ as a function of $\xi \circ$

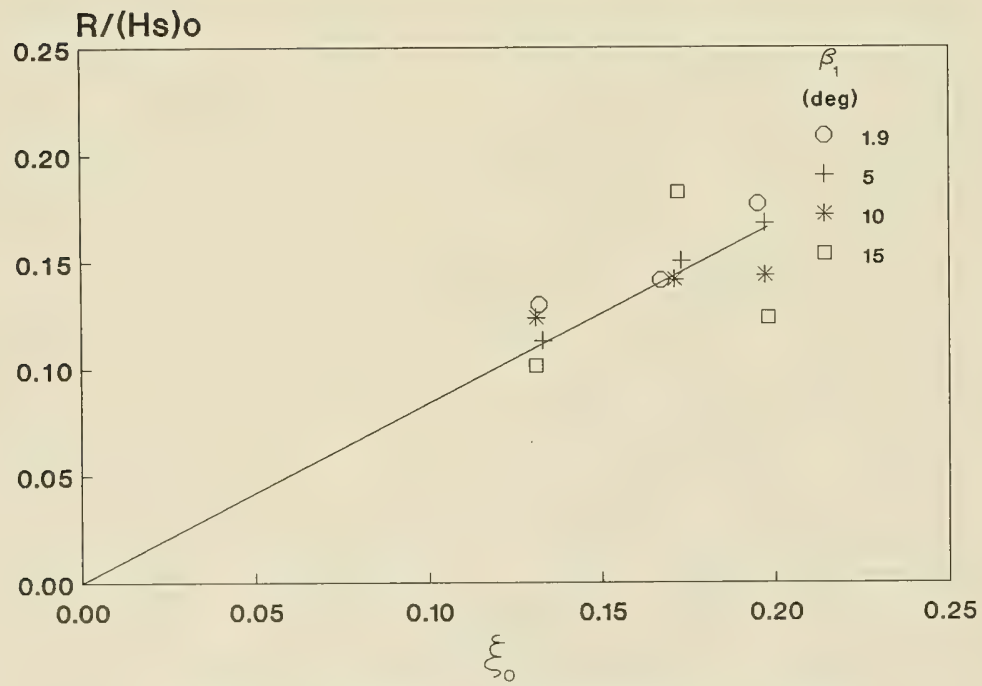

Figure 77. $\mathrm{R} /\left(\mathrm{H}_{\mathrm{o}}\right)_{\mathrm{s}}$ as a function of $\xi_{0}$ using $\mathrm{m}=1 / 30$ as the predominant angle 
168. Runup normalized by $H_{\text {rms }}$ for irregular waves was plotted versus seaward bar angle in Figure 78. Runup values for monochromatic waves were included in the plot to illustrate the differences between wave types. Runup of irregular waves was higher than that of monochromatic waves of equivalent deepwater wave steepness, which is attributed to some waves overtaking others and causing a higher uprush, and the back wash of large waves obliterating the incoming smaller waves, which results in measurements of only higher runup events.

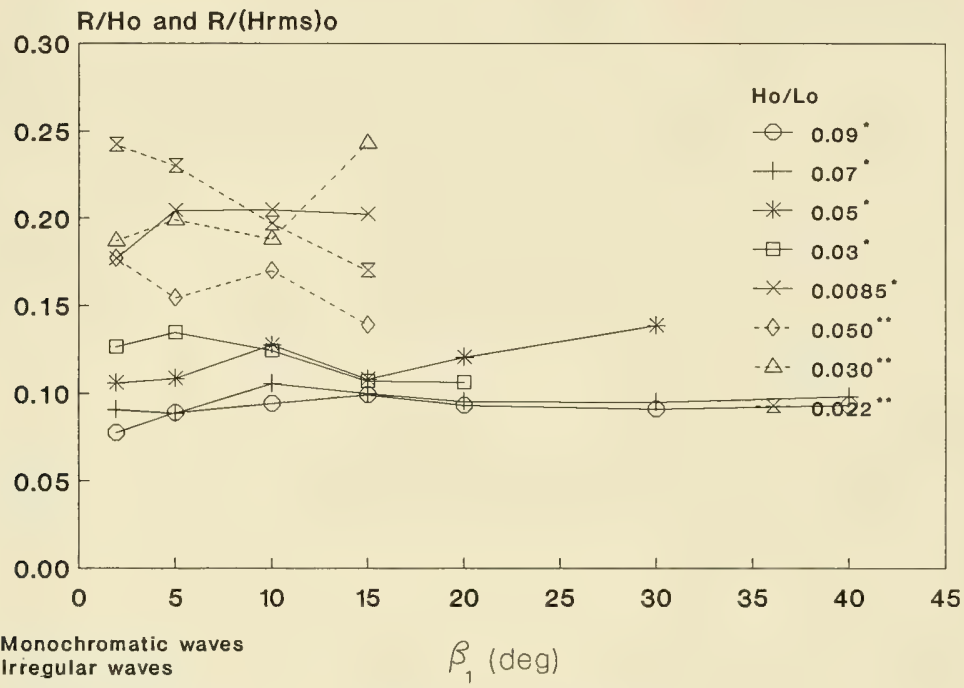

Figure 78. $\mathrm{R} / \mathrm{H}_{\mathrm{rms}}$ as a function of $\beta_{1}$

\section{Measurement Errors}

169. All tests were conducted with utmost care. However, some error was introduced because of equipment limitations.

170. A description of the data collection system used can be found in Turner and Durham (1984). Wave rods were calibrated before the first test of the day using the microcomputer that collected the wave data. Calibration began when a signal was sent from the microcomputer to the motors attached to the wave rods. The signal caused the motors to move the wave rods a predetermined vertical distance, and a voltage reading was taken by the rod at the 
depth. The procedure was repeated until readings had been made for 11 positions over the calibration range of the rods. The voltage readings were converted to vertical distances from the horizontal datum, SWL, and compared with the known physical position of the rod at each reading. The voltage readings of the rods were accepted if the maximum deviation for each of the 11 positions was less than $0.01 \mathrm{ft}$. The maximum deviation was usually less than $0.01 \mathrm{ft}$, and deviations greater than this were typically caused by residue on the rods. In these cases, the rod was cleaned, and the procedure was repeated for the individual rods that exceeded the tolerance.

171. The SWL was measured by a point gage, graduated to $0.001 \mathrm{ft}$, 1ocated in the horizontal section of the tank. The water depth was checked before conducting each test. Measurements of water depth at gages and at the bar were made with a rule that was accurate to $0.05 \mathrm{ft}$. The same rule was used for measuring seaward and shoreward faces during construction of the bars. All measurements during video analysis were made by scaling the 2-in. grid as it appeared on the monitor to $1 / 4 \mathrm{in.,}$ which gave an error of $\pm 1 / 8 \mathrm{in}$.

172. Monochromatic waves were analyzed and averaged for 15 waves from the wave record, and measurements from videotapes were averaged for 10 waves. Although the waves were monochromatic (constant period) and regular (constant height), it was important to average the measured quantities to reduce fluctuations caused by reflections and currents present in the tank. It was also critical to limit the number of waves averaged to those that were not reflected from the wave board.

173. To illustrate the importance of averaging values, breaker depth index was plotted as a function of $\mathrm{H}_{0} / \mathrm{L}_{0}$ for five tests, as shown in Figure 79. The average value of $\gamma_{b}$ is marked by the symbol, and the range between maximum and minimum values of $\gamma_{b}$ computed from the 10 measured values of each test is shown by the vertical line at each wave steepness. It is seen that differences of 15 percent in individual values occurred despite care to eliminate wave reflection and seiching. The considerable scatter in the data, often much more than 15 percent, shown in many figures of this report is attributable in part to the difficulty in defining the given quantity, such as breaking of collapsing waves, and not to direct measurement. The process of averaging 10 values as done in the present study helped to reduce scatter due to individual wave variation and thus presented a more realistic picture of the variability in the developed relationships. 


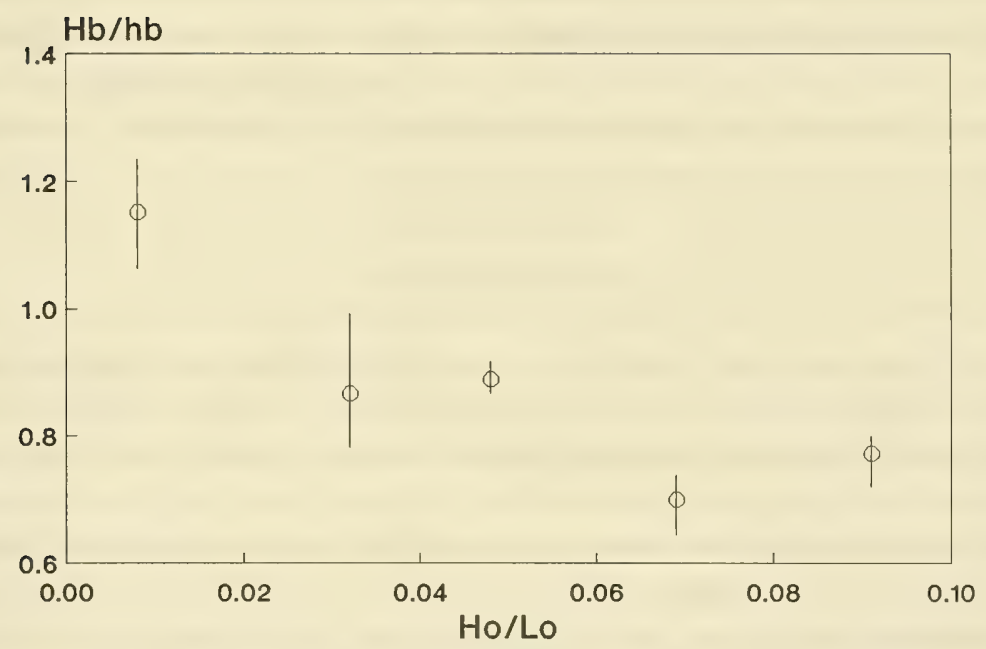

Figure 79. Range between maximum and minimum values of $\gamma_{b}$ as a function of $H_{o} / L_{\circ}$ 
174. The purpose of this study was to examine the macroscale features of wave breaking over bars and artificial reefs in a wave tank. All major experiments on wave breaking prior to this one were conducted on plane beaches, and relationships for breaker indices and plunge distances developed from these studies were expected to be invalid for complex profiles. The present experiments demonstrated that waves break differently (their forms are different) on a barred profile as compared with on a plane slope, and wave breaking properties such as breaker index and plunge distance also differ. Equations were developed incorporating seaward bar angle and deepwater wave steepness for describing breaker indices, plunge distance, splash distance, penetration distance, and vortex area. New relationships were also developed for breaker index and plunge distance based on a large data set developed from previous plane-slope experiments.

\section{Monochromatic Tests}

175. Breaker type transition values for spilling and plunging waves over barred profiles were lower than the commonly accepted values given by Battjes (1975) that apply to plane slopes. A strong return flow was observed if seaward bar angles were steep or wave steepness was low. The return flow influenced breaker type by causing the wave to "trip" over the bar, rather than to break as a result of becoming unstable due to limiting depth. The return flow was also strong if the shoreward bar angle was horizontal (terraced bottom). Depth-limited or well-behaved breaking occurred for conditions in which the seaward length of the bar was at least 75 percent as long as the wavelength at breaking. Complex breaking occurred for conditions in which the bar faces were steep, and the seaward bar length was shorter than the breaking wavelength.

176. Breaker depth index was greatly influenced by return flow, based on visual observations. Breaker depth data were scattered for collapsing waves and for plunging waves with large values of the surf similarity parameter. A relationship was developed for breaker depth index for plunging and spilling waves having seaward bar angles less than $10 \mathrm{deg}$, which restricted the analysis to depth-limited breaking (small return flow). The seaward bar 
angle $\beta_{1}$ created under natural conditions is typically gentler than $10 \mathrm{deg}$. The relationship for barred profiles was compared with the equation developed in this study for plane slopes. The plane-slope equation gave smaller values of breaker depth index than the barred profile equation for $\beta_{1}=5$ deg for low wave steepness and all wave steepnesses for 10-deg seaward angles. The plane-slope equation gave larger breaker depth values than the barred profile equation for $\beta_{1}=5 \mathrm{deg}$ and $0.02<\mathrm{H}_{\mathrm{o}} / \mathrm{L}_{\mathrm{o}}<0.09$.

177. Breaker height index was a stabler parameter for conditions involving a strong return flow. Breaker height as a function of deepwater wave steepness gave the same trend for bars as data collected on plane slopes. An equation for the experimental data was expressed in the same form as the analogous plane-slope equation. Breaker height decreased for $\beta_{1}>10 \mathrm{deg}$ at high wave steepnesses, but if wave steepness was low, breaker heights increased as $\beta_{1}$ increased. For high-steepness waves and steep bars, wave shoaling was mostly caused by the plane slope because the bars were short and had little effect on the waves. Return flow was not strong for these conditions. If wave steepness was low and bars steep, a strong return flow was present. A heuristic derivation was made to illustrate breaking wave height increases as return velocity increases.

178. Plunge distance normalized by breaking wave height was relatively constant for larger values of the surf similarity parameter, but increased at smaller values of the surf similarity parameter. Plunge distance was shorter by a factor of approximately 2 if bars were present as compared with the analogous plane-slope case, because breaker type differs with bars present. Plunge distance normalized by deepwater wave height showed no dependence on the surf similarity parameter, which indicates the distance traveled by the plunging wave depends on the local (breaking) wave height.

179. The average of plunge distance divided by splash distance was approximately unity, which agrees with findings of Galvin (1969) for plane slopes, but the plunge-to-splash distance ratio increased for smaller valued surf similarity parameters. Plunge distance was also greater for smaller surf similarity values. Splash distance normalized by deepwater and breaking wave height was essentially constant if expressed as a function of surf similarity, but the data were widely scattered.

180. For barred profiles, the total distance traveled by the wave crest from the break point was called the penetration distance. Penetration 
distance was found to increase for small values of the surf similarity parameter. Penetration distance normalized by deepwater wave height showed no dependency on the surf similarity parameter and appears to be dependent on local wave height only.

181. Vortex area was measured at the plunge point for 34 tests with the seaward bar face angle $\beta_{1} \leq 10 \mathrm{deg}$. The plunge point was chosen because of its uniqueness to every breaking wave, and it is the point of vortex formation. Vortex area was found to increase with values of the surf similarity parameters and approach an asymptotic value for waves in the collapsing region.

182. Wave reflection was measured seaward of the bar for all tests and was found to increase for steeper bars, which was expected, although reflection did not notably increase with bar slope. Reflection coefficients from the experiment were compared with relationships of Miche (1951) and Battjes (1975) developed for plane slopes. The equation of Miche overpredicted reflection for most of the experimental data. The Battjes equation predicts increasing reflection with the surf similarity parameter; however, measured values plotted as near-constant and were independent of the surf similarity parameter. Reflection coefficients were also correlated with the reef reflection parameter of Ahrens (1987) developed for reef-type breakwaters. The Ahrens equation gave better agreement, but the equation did not fully reproduce the trend. A parameter to quantify wave reflection from bars and reefs was not identified, and additional study is required.

183. Wave runup was measured for all tests. Runup was normalized by deepwater wave height and plotted as a function of seaward bar angle, but seaward angle showed little effect on runup. Wave runup was also constant, but scattered, if plotted versus the surf similarity parameter; however, runup was found to increase with the surf similarity parameter if the beach slope of $1 / 30$ was used to compute $\xi_{0}$ instead of seaward bar angle. The average runup normalized by $H_{\circ}$ was $0.76 \xi_{0}$, whereas Battjes (1975) found that $R / H_{0}$ $=1.0 \xi_{0}$. Lower measured values of runup could result from additional energy dissipation caused by bars; however, runup was also lower than predicted by Battjes for the present plane-slope tests. A complete analysis of wave height decay and setup is required to reach a relationship for runup for barred profiles. Wave data were collected through the surf zone and are presented in Appendix C. 


\section{Irregular Wave Tests}

184. Maximum breaker height from irregular wave tests was compared with the predictive expression of Goda (1975) using $A=0.18$. Maximum wave height was predicted well for $\beta_{1} \leq 10 \mathrm{deg}$. Significant wave height was also compared with the Goda expression with $A=0.12$, which also gave good results for $\beta_{1} \leq 10 \mathrm{deg}$. The minimum coefficient specified by Goda $(A=0.12)$ was reduced by $\sqrt{2}$ to predict values for rms wave height based on a Rayleigh distribution, which gave $A=0.09$. The equation reasonably predicted wave heights for $\beta_{1} \leq 10 \mathrm{deg}$ and overpredicted wave heights for $\beta_{1}=15 \mathrm{deg}$.

185. Wave height-to-water depth ratio was plotted as a function of horizontal distance from the shoreline for irregular waves. The ratio was not constant through the inner surf zone, but increased uniformly into shallow water for plane-sloping beaches and was double peaked for single barred profiles.

186. Irregular wave runup was normalized by $\left(\mathrm{H}_{\mathrm{s}}\right)_{0}$, plotted as a function of the surf similarity parameter, and compared with the expression of Ahrens (1981). The Ahrens expression predicted runup for the plane-slope tests well, but overpredicted runup with bars in the tank. The predicted values of Ahrens showed good agreement with the measured values if the foreshore beach slope $(1 / 30)$ was used to compute the surf similarity parameter rather than seaward bar angle. Runup thus appears to depend mainly on the foreshore slope and not on the presence of a bar.

\section{Recommendations for Future Study}

187. This study verified the strategy of using solid but adjustable bars for examining wave breaking on realistic nearshore bottom shapes. It is recommended to continue the line of study to more exact replication of bar forms and sizes, although gently sloping bars will require considerable resources, equipment, and effort to build and emplace.

188. It was clearly demonstrated that bars alter the characteristics of breaking waves. This is due to the steeper seaward bar slope, volume of water shoreward of the bar, and the speed of the return flow. In future 
experiments, the speed of the return flow should be measured and related to the breaking wave properties.

189. Commonly accepted formulae for runup and wave reflection did not well describe the data generated in this study. Further work is needed to investigate more fully these quantities.

190. A next logical step in this experiment program would be to emphasize irregular wave breaking. The video technique relied upon extensively in the present study will probably be relegated to providing supplementary qualitative information, with quantitative data requirements necessitating a dense array of wave gages or other, more automated measurement procedures. 
Ahrens, J. P. 1979. "Irregular Wave Runup," Proceedings of Coastal Structures '79, American Society of Civil Engineers, pp 998-1019.

1981. "Irregular Wave Runup on Smooth Slopes," Coastal Engineering Technical Aid No. 81-17, US Army Engineer Waterways Experiment Station, Coastal Engineering Research Center, Vicksburg, MS.

1987. "Characteristics of Reef Breakwaters," Technical Report CERC-87-17, US Army Engineer Waterways Experiment Station, Coastal Engineering Research Center, Vicksburg, MS.

Ahrens, J. P., and Titus, M. F. 1985. "Wave Runup Formulas for Smooth Slopes," Journal of Waterway. Port. Coastal and Ocean Engineering, American Society of Civil Engineers, Vo1 111, No. 1, pp 128-133.

Battjes, J. A. 1973. "Set-Up due to Irregular Waves," Proceedings of the 13th Coastal Engineering Conference, American Society of Civil Engineers, pp 1993-2004.

1975. "Surf Similarity," Proceedings of the 14th Coastal Engineering Conference, American Society of Civil Engineers, pp 466-480.

Battjes, J. A., and Janssen, J. P. F. M. 1979. "Energy Loss and Setup due to Breaking of Random Waves," Proceedings of the 16th Coastal Engineering Conference, American Society of Civil Engineers, pp 569-587.

Bowen, A. J., Inman, D. L., and Simmons, V. P. 1969. "Wave 'Set-Down' and Set-Up," Journal of Geophysical Research, Vol 73, No. 8, pp 2569-2577.

Collins, J. I. 1971. "Probabilities of Breaking Wave Characteristics," Proceedings of the 12 th Coastal Engineering Conference, American Society of Civil Engineers, pp 399-414.

Collins, J. I., and Weir, W. 1969. "Probabilities of Wave Characteristics in the Surf Zone," Tetra Tech Report \#TC-149, Pasadena, CA.

Dally, W. R. 1980. "Numerical Model for Beach Profile Evolution," M. S. Thesis, Department of Civil Engineering, University of Delaware, Newark, DE.

Dally, W. R., Dean, R. G., and Dalrymple, R. A. 1985a. "A Model for Breaker Decay on Beaches," Proceedings of the 19th Coastal Engineering Conference, American Society of Civil Engineers, pp 82-98.

1985b. "Wave Height Variation Across Beaches of Arbitrary Profile," Journal of Geophysical Research, Vo1 90, No. C6, pp 11917-11927.

Dane1, P. 1952. "On the Limiting Clapotis," Gravity Waves, Circular 521, National Bureau of Standards, pp 35-45.

Divoky, D., Le Méhauté, B., and Lin, A. 1970. "Breaking Waves on Gentle Slopes," Journal of Geophysical Research, Vol 75, No. 9, pp 1681-1692.

Douglass, S. L., and Weggel, J. R. 1989. "Laboratory Experiments on the Influence of Wind on Nearshore Wave Breaking," Proceedings of the 21st Coastal Engineering Conference, American Society of Civil Engineers, pp 632-643.

Duncan, J. H. 1981. "An Experimental Investigation of Breaking Waves Produced by a Towed Hydrofoil," Proceedings of the Royal Sociaty of London, Vol A 377, pp 331-348. 
Ebersole, B. A. 1987. "Measurement and Prediction of Wave Height Decay in the Surf Zone," Proceedings of the Coastal Hydrodynamics Conference, American Society of Civil Engineers, pp 1-16.

Ebersole, B. A., and Hughes, S. A. 1987. "DUCK85 Photopole Experiment," Miscellaneous Paper CERC-87-18, US Army Engineer Waterways Experiment Station, Coastal Engineering Research Center, Vicksburg, MS .

Fowler, J. E., and Smith, E. R. 1986. "Evaluation of Movable-Bed Modeling Guidances," Proceedings of the Third International Symposium on River Sedimentation, School of Engineering, University of Mississippi, pp 1793-1802.

Galvin, C. J. 1968. "Breaker Type Classification on Three Laboratory Beaches," Journal of Geophysical Research, Vol 73, No. 12, pp 3651-3659.

1969. "Breaker Travel and Choice of Design Wave Height," Journal of the Waterways and Harbors Division, American Society of Civil Engineers, Vol 95, No. WW2, pp 175-200.

1970. "Finite-Amplitude, Shallow Water-Waves of Periodically Recurring Form," Proceedings of the Symposium on Long Waves, pp 1-32.

Goda, Y. 1970. "A Synthesis of Breaker Indices, " Transactions of Japanese Society of Civil Engineers, Vol 2, Part 2, pp 227-230.

1975. "Irregular Wave Deformation in the Surf Zone," Coastal Engineering in Japan, Vol 18, pp 13-26.

Goda, Y., and Suzuki, Y. 1977. "Estimation of Incident and Reflected Waves in Random Wave Experiments," Proceeding of the 15th Coastal Engineering Conference, American Society of Civil Engineers, pp 828-845.

Hamada, T. 1963. "Breakers and Beach Erosion," Port and Harbour Research Institute, Ministry of Transport, Yokosuka, Japan.

Holman, R. A., and Sallenger, A. H., Jr. 1985. "Setup and Swash on a Natural Beach," Journal of Geophysical Research, Vo1 90, No. C1, pp 945-953.

Horikawa, K., and Kuo, C. 1967. "A Study on Wave Transformation Inside the Surf Zone," Proceedings of the 10th Coastal Engineering Conference, American Society of Civil Engineers, pp 235-249.

Hughes, S. A. 1983. "Movable-Bed Modeling Law for Coastal Dune Erosion," Journal of Waterway, Port, Coastal and Ocean Engineering, American Society of Civil Engineers, Vol 109, No. 2, pp 164-179.

Hunt, I. A. 1959. "Design of Seawalls and Breakwaters," Journal of the Waterways and Harbors Division, American Society of Civil Engineers, Vol 85, No. WW3, pp 123-152.

Ippen, A. T., and Kulin, G. 1955. "The Shoaling and Breaking of the Solitary Wave," Proceedings of the 5th Coastal Engineering Conference, American Society of Civil Engineers, pp 27-47.

Iribarren, C. R., and Nogales, C. 1947. "Protection des Ports," Section II, Comm. 4, XVIIth International Navigation Congress, Lisbon, Portugal, pp 31-80. Iversen, H. W. 1952. "Laboratory Study of Breakers," Gravity Waves, Circular 52, US Bureau of Standards, pp 9-32. 
Iwagaki, Y., Sakai, T., Tsukioka, K., and Sawai, N. 1974. "Relationship Between Vertical Distribution of Water Particle Velocity and Type of Breakers on Beaches," Coastal Engineering in Japan, Vol 17, pp 51-58.

Jen, Y., and Lin, P.-M. 1971. "Plunging Wave Pressures on a Semi-Cylindrica1 Tube," Proceedings of the 12th Coastal Engineering Conference, American Society of Civil Engineers, pp 1469-1490.

Kajima, R., Shimizu, T., Maruyama, K., and Saito, S. 1983. "Experiments of Beach Profile Change with Large Wave Flume," Proceedings of the 18th Coastal Engineering Conference, American Society of Civil Engineers, pp 1385-1404.

Kamphuis, J. W., and Mohamed, N. 1978. "Runup of Irregular Waves on Plane Smooth Slope," Journal of the Waterway, Port, Coastal, and Ocean Division, Vo1 104, No. WW2, pp 135-146.

Keulegan, G. H. 1945. "Depths of Offshore Bars," Beach Erosion Board, Engineering Notes No. 8, US Army Engineer Waterways Experiment Station, Coastal Engineering Research Center, Vicksburg, MS.

Komar, P. D., and Gaughan, M. K. 1973. "Airy Wave Theory and Breaker Height Prediction," Proceedings of the 13th Coastal Engineering Conference, American Society of Civil Engineers, pp 405-418.

Kraus, N. C., and Larson, M. 1988. "Beach Profile Change Measured in the Tank for Large Waves 1956-1957," Technical Report CERC-88-6, US Army Engineer Waterways Experiment Station, Coastal Engineering Research Center, Vicksburg, MS .

Kuo, C. T. 1965. "A Study on Wave Transformation After Breaking," M. S. Thesis, Department of Civil Engineering, University of Tokyo, Tokyo, Japan. (in Japanese).

Kuo, C. T., and Kuo, S. T. 1974. "Effect of Wave Breaking on Statistical Distribution of Wave Heights," Proceedings Civil Engineering Oceans,

pp 1211-1231.

Larson, M., and Kraus, N. C. 1989. "SBEACH: Numerical Model for Simulating Storm-Induced Beach Change, Report 1, Empirical Foundation and Model Development," Technical Report CERC-89-9, US Army Engineer Waterways Experiment Station, Coastal Engineering Research Center, Vicksburg, MS.

Larson, M., Kraus, N. C., and Sunamura, T. 1989. "Beach Profile Change: Morphology, Transport Rate, and Numerical Simulation," Proceedings of the 21st Coastal Engineering Conference, American Society of Civil Engineers, pp 1295-1309.

LeMéhauté, B. 1963. "On Non-Saturated Breakers and the Wave Run-Up," Proceedings of the 8 th Coastal Engineering Conference, American Society of Civil Engineers, pp 77-92.

Lepetit, J. P., and Leroy, J. 1977. "Etalonnage Sedimentologique du Modele Reduit d'Ensemble," Rap. 14 (Avant-Port ouest de Dunkerque), Laboratoire National D'Hydraulique, Electricite de France, Chatou, France.

Longuet-Higgins, M. S., and Stewart, R. W. 1964. "Radiation Stress in Water Waves; A Physical Discussion with Application," Deep Sea Research, Vol 11, No. 4, pp 529-562. 
Mase, H., and Iwagaki, Y. 1985. "Run-Up of Random Waves on Gentle Slopes," Proceedings of the 19 th Coastal Engineering Conference, American Society of Civil Engineers, pp 593-609.

Maruyama, K., Sakakiyama, T., Kajima, R., Saito, S., and Shimizu, T. 1983. "Experimental Study on Wave Height and Water Particle Velocity Near the Surf Zone Using a Large Wave Flume," Civil Engineering Laboratory Report No. 382034, The Central Research Institute of Electric Power Industry, Chiba, Japan. (in Japanese).

McCowan, J. 1891. "On the Solitary Wave," Philosophical Magazine, 5th Series, Vo1 32, No. 194, pp 45-58.

McLellan, T. N. 1990. "Nearshore Mound Construction Using Dredged Material," Journal of Coastal Research, Specialty Issue No. 6, Rational Design of Mound Structures.

Miche, M. 1951. "Le Pouvoir Reflechissant des Ouvrages Maritimes Exposes a 1'Action de la Houle" Annals des Ponts et Chaussees, 12le Annee, pp 285-319 (translated by Lincoln and Chevron, University of California, Berkeley, Wave Research Laboratory, Series 3, Issue 363, June, 1954).

Miche11, J. H. 1893. "On the Highest Waves in Water," Philosophical Magazine, 5th Series, Vol 36, pp 430-437.

Miller, R. L. 1976. "Role of Vortices in Surf Zone Prediction: Sedimentation and Wave Forces," Beach and Nearshore Sedimentation, Society of Economic Paleontologists and Mineralogists, Special Publication No. 24, pp 92-114.

Mizuguchi, M. 1981. "An Heuristic Model of Wave Height Distribution in Surf Zone," Proceedings of the 17th Coastal Engineering Conference, American Society of Civil Engineers, pp 278-289.

Moraes, C. 1971. "Experiments of Wave Reflexion on Impermeable Slopes," Proceedings of the 12th Coastal Engineering Conference, American Society of Civil Engineers, pp 509-521.

Munk, W. H. 1949. "The Solitary Wave Theory and Its Application to Surf Problems," Annals of the New York Academy of Sciences, Vol 51.

Noda, E. K. 1972. "Equilibrium Beach Profile Scale-Model Relationship," Journal of the Waterways, Harbors and Coastal Engineering Division, American Society of Civil Engineers, Vol 98, No. WW4, pp 511-528.

Patrick, D. A, and Wiegel, R. L. 1954. "Amphibian Tractors in the Surf," Proceedings of the First Conference on Ships and Waves, American Society of Civil Engineers, pp 397-422.

Phillips, O. M. 1977. The Dynamics of the Upper Ocean, Cambridge University Press, London.

Pratte, T. 1989. "Patagonia Reef: One Step at a Time", Making Waves, The Surfrider Foundation, Vol 5, No. 1, p 5.

Reid, R. O., and Bretschneider, C. L. 1953. "Surface Waves and Offshore Structures," Technical Report No. 53-10, Texas AdM University, College Station, TX.

Saeki, H., and Sasaki, M. 1973. "A Study of the Deformation of Waves After Breaking (1)," Proceedings of the 20th Japanese Conference on Coastal Engineering, Japan Society of Civil Engineers, pp 559-564. (in Japanese). 
Sallenger, A. S., Jr., Holman, R. A., and Birkemeier, W. A. 1985. "StormInduced Response of a Nearshore-Bar System, " Marine Geology, Vol 64, pp 237-257.

Sallenger, A. S., Jr., and Howd, P. A. 1989. "Nearshore Bars and the BreakPoint Hypothesis," Coastal Engineering, Vol 12, pp 301-313.

Sasaki, M., and Saeki, H. 1974. "A Study of the Deformation of Waves After Breaking (2)," Proceedings of the 21st Japanese Conference on Coastal Engineering, Japan Society of Civil Engineers, pp 39-44. (in Japanese).

Savage, R. P. 1958. "Wave Runup on Roughened and Permeable Slopes, " Journal of the Waterways and Harbor Division, American Society of Civil Engineers, Vo1 84, No. WW3, pp 1-38.

Saville, T., Jr. 1955. "Data on Wave Run-Up and Overtopping of Shore Structures," Technical Memorandum No. 64, Beach Erosion Board, US Army Engineer Waterways Experiment Station, Coastal Engineering Research Center, Vicksburg, MS .

1956. "Wave Run-Up on Shore Structures," Journal of the Waterways and Harbors Division, American Society of Civil Engineers, Vol 82, WW2, pp 1-14.

. 1957. "Scale Effects in Two Dimensional Beach Studies," Proceedings 7 th International Association of Hydraulic Research Congress, pp A3.1-A3.10.

Sawaragi, T., and Iwata, K. 1974. "Turbulence Effect on Wave Deformation After Breaking," Coastal Engineering in Japan, Vol 17, pp 39-49.

Sawaragi, T., Deguchi, I., and Park, S. 1989. "Reduction of Wave Overtopping Rate by the Use of Artificial Reefs," Proceedings of the 21st Coastal Engineering Conference, American Society of Civil Engineers, pp 335-349.

Seelig, W. N. 1979. "Estimating Nearshore Significant Wave Height for Irregular Waves, " Coastal Engineering Technical Aid No. 79-5, US Army Engineer Waterways Experiment Station, Coastal Engineering Research Center, Vicksburg, MS.

. 1980. "Maximum Wave Heights and Critical Water Depths for Irregular Waves in the Surf Zone," Coastal Engineering Technical Aid No. 80-1, US Army Engineer Waterways Experiment Station, Coastal Engineering Research Center, Vicksburg, MS .

Seelig, W. N., Ahrens, J. P., and Grosskopf, W. G. 1983. "The Elevation and Duration of Wave Crests," Miscellaneous Report 83-1, US Army Engineer Waterways Experiment Station, Coastal Engineering Research Center, Vicksburg, MS.

Shore Protection Manual. 1977. 3rd Ed., 3 Vol, US Army Engineer Waterways Experiment Station, Coastal Engineering Research Center, US Government Printing Office, Washington, DC.

Singamsetti, S. R., and Wind, H. G. 1980. "Characteristics of Breaking and Shoaling Periodic Waves Normally Incident on to Plane Beaches of Constant Slope," Report M1371, Delft Hydraulic Laboratory, Delft, The Netherlands.

Smith, J. M., and Kraus, N. C. 1988. "An Analytical Model of Wave-Induced Longshore Current Based on Power Law Wave Height Decay," Miscellaneous Paper CERC-88-3, US Army Engineer Waterways Experiment Station, Coastal Engineering Research Centex, Vicksburg, MS . 
Stevens, J. C., Bardsley, C. E., Lane, E. W., and Straub, L. G. 1942. "Hydraulic Models," Manuals on Engineering Practice, No. 25, American Society of Civil Engineers, New York.

Stive, M. J. F. 1984. "Energy Dissipation in Waves Breaking on Gentle Slopes," Coastal Engineering, Vol 8, pp 99-127.

Engineering, Vol 9, pp 151-158.

Street, R. L., and Camfield, F. E. 1967. "Observation and Experiments on Solitary Wave Deformation," Proceedings of the 10th Coastal Engineering Conference, American Society of Civil Engineers, pp 284-293.

Sunamura, T. 1981. "A Laboratory Study of Offshore Transport of Sediment and a Model for Eroding Beaches," Proceedings of the 17 th Coastal Engineering Conference, American Society of Civil Engineers, pp 1051-1070.

1982. "Determination of Breaker Height and Depth in the Field," Annual Report of the Institute of Geoscience, University of Tsukuba, Tsukuba, Japan, No. 8, pp 53-54.

1987. "Wave-Induced Geomorphic Response of Eroding Beaches with Special Reference to Seaward Migrating Bars," Proceedings of Coastal Sediments 187, American Society of Civil Engineers, pp 788-801.

Sunamura, T., and Horikawa, K. 1975. "Two-Dimensional Beach Transformation Due to Waves," Proceedings of the 14th Coastal Engineering Conference, American Society of Civil Engineers, pp 920-938.

Suquet, F. 1950. "Experimental Study on the Breaking of Waves," La Houille Blanche, No. 3.

Svendsen, I. A. 1984. "Wave Heights and Set-Up in a Surf Zone," Coastal Engineering, Vo1 8, pp 303-329.

1985. "Wave Attenuation and Set-Up on a Beach," Proceedings of the 19th Coastal Engineering Conference, American Society of Civil Engineers, pp 54-69.

Thompson, E. F., and Vincent, C. L. 1984. "Shallow Water Wave Height Parameters," Journal of Waterway, Port, Coastal and Ocean Engineering, American Society of Civil Engineers, Vol 110, No. 2, pp 293-299.

Thornton, E. B., and Guza, R. T. 1983. "Transformation of Wave Height Distribution," Journal of Geophysical Research, Vol 88, No. C10, pp 5925-5938.

Thornton, E. B., Wu, C. S., and Guza, R. T. 1985. "Breaking Wave Design Criteria," Proceedings of the 19th Coastal Engineering Conference, American Society of Civil Engineers, pp 31-41.

Turner, K. A., and Durham, D. L. 1984. "Documentation of Wave-Height and Tidal Analysis Programs for Automated Data Acquisition and Control Systems," Miscellaneous Paper HL-84-2, US Army Engineer Waterways Experiment Station, Vicksburg, MS.

Van Dorn, W. G. 1977. "Set-Up and Run-Up in Shoaling Breakers," Proceedings of the 15th Coastal Engineering Conference, American Society of Civil Engineers, pp 738-751. 
Van Oorschot, J. H., and d'Angremond, K. 1969. "The Effect of Wave Energy Spectra on Wave Runup," Proceedings of the 11th Coastal Engineering Conference, American Society of Civil Engineers, pp 888-900.

Vellinga, P. 1982. "Beach and Dune Erosion During Storm Surges," Coastal Engineering, Vol 6, No. 4, pp 361-387.

Visser, P. J. 1982. "The Proper Longshore Current in a Wave Basin," Report No. 82-1, Laboratory of Fluid Mechanics, Department of Civil Engineering, Delft University of Technology, Delft, The Netherlands.

Walker, J. R. 1974a. "Recreational Surf Parameters," Technical Report No. 30, University of Hawaii, Look Lab-73-30, Honolulu, HI. . 1974b. "Wave Transformations Over a Sloping Bottom and Over a Three-Dimensional Shoal," Miscellaneous Report No. 11, University of Hawaii, Look Lab-75-11, Honlulu, HI.

Wegge1, J. R. 1972. "Maximum Breaker Height," Journal of the Waterways. Harbors and Coastal Engineering Division, American Society of Civil Engineers, Vol 98, No. WW4, pp 529-548.

Wegge1, J. R., and Maxwe11, W. H. C. 1970. "Experimental Study of Breaking Wave Pressures," OTC-1244, Second Annual Offshore Technology Conference.

Weisher, L. L., and Byrne, R. J. 1979. "Field Study of Breaking Wave Characteristics," Proceedings of the 16th Coastal Engineering Conference, American Society of Civil Engineers, pp 487-506. 

1. This appendix consists of data generated in the present study and data obtained from previous laboratory experiments conducted with periodic waves.

2. Results from the present study (Tables $A 1$ and $A 2$ ) represent average values of 10 to 15 consecutive waves. Analysis of the data was begun after waves reflected off the concrete slope $(m=1 / 30)$ and was ended before reflections from the wave board had returned to the bar.

3. Data from previous studies (Tables A3-A13) were selected from experiments in which the slope was planar and fixed, deepwater steepness was given or could be calculated from the data, and no structures were present in the wave tank that would alter wave breaking. 


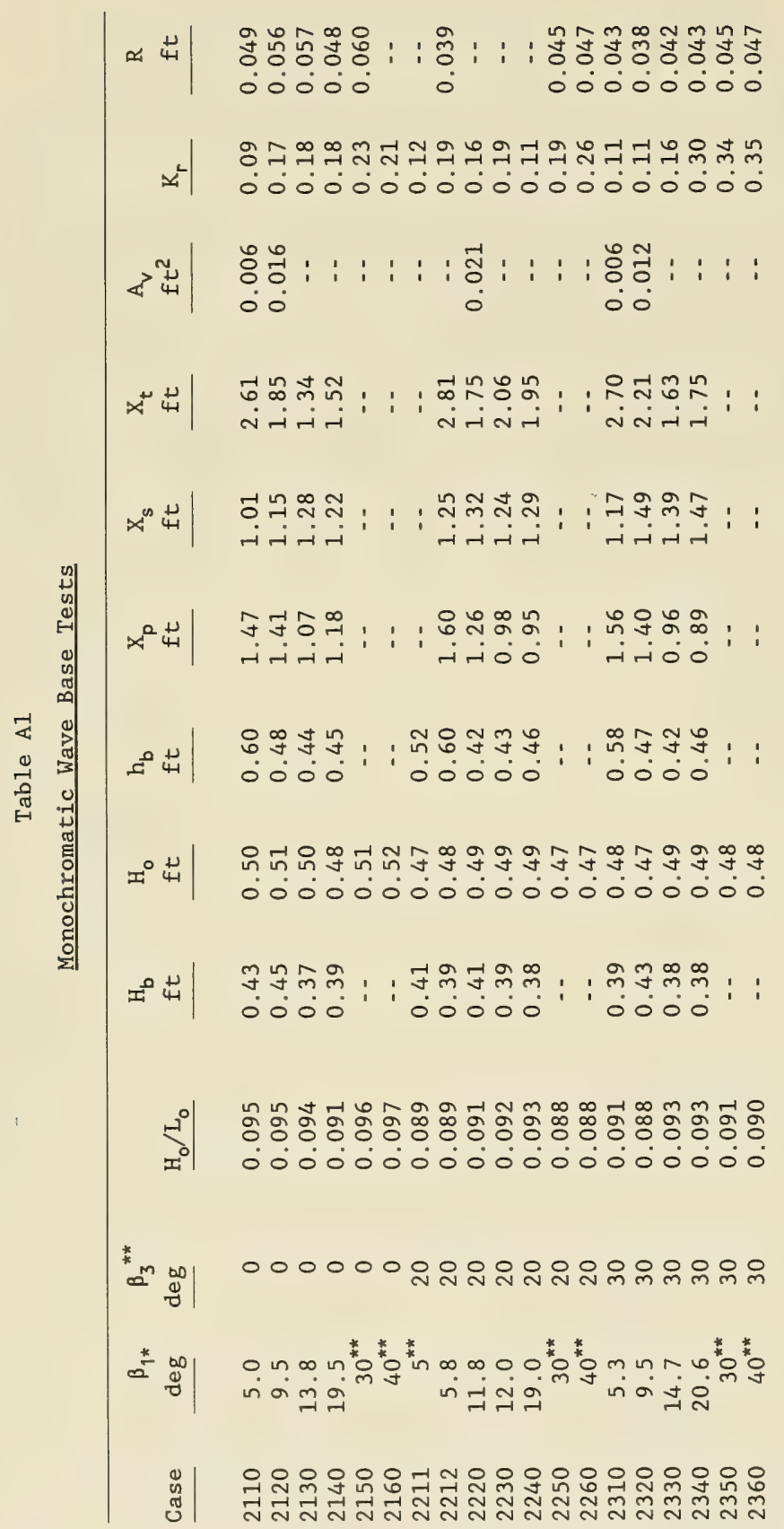




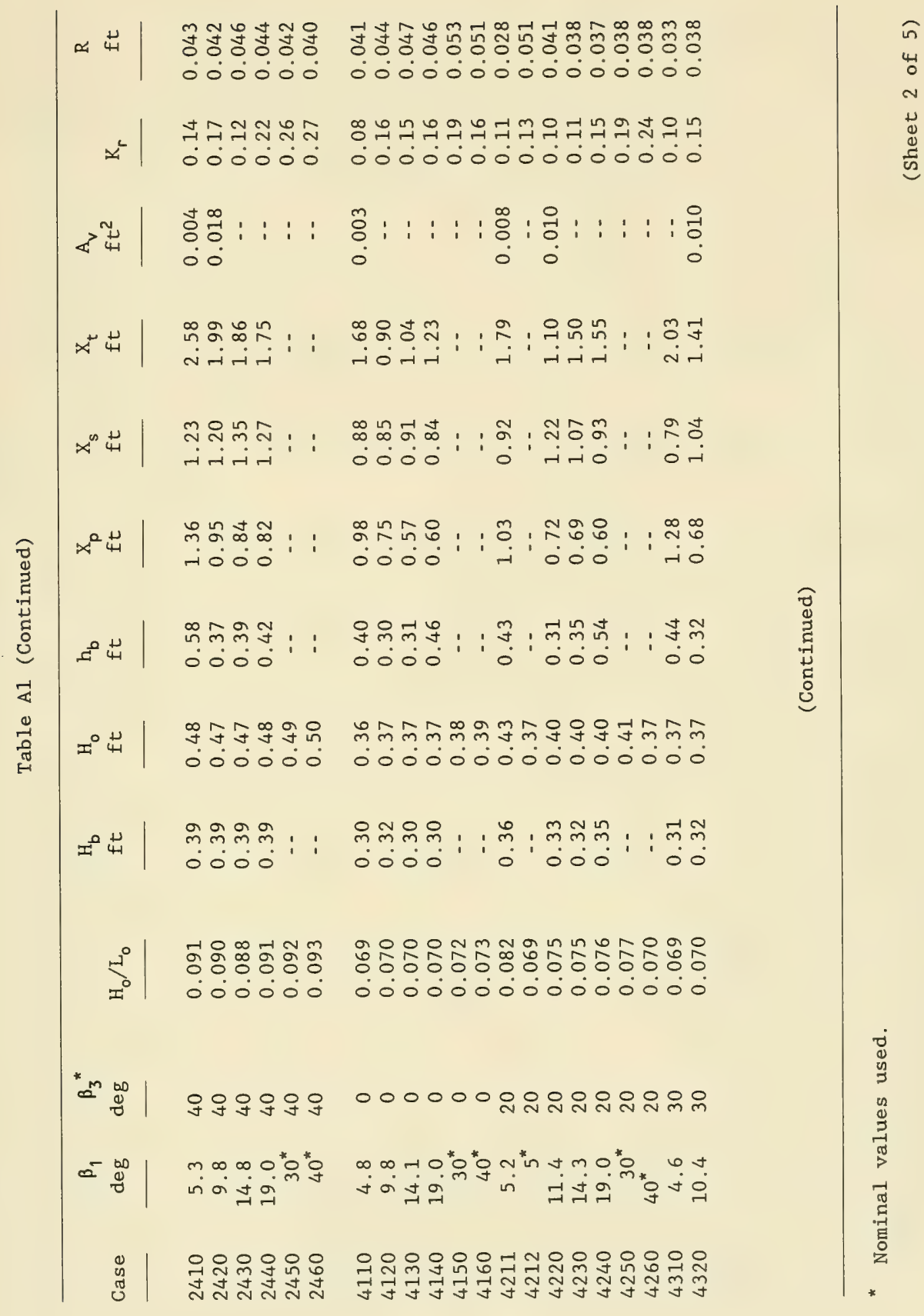




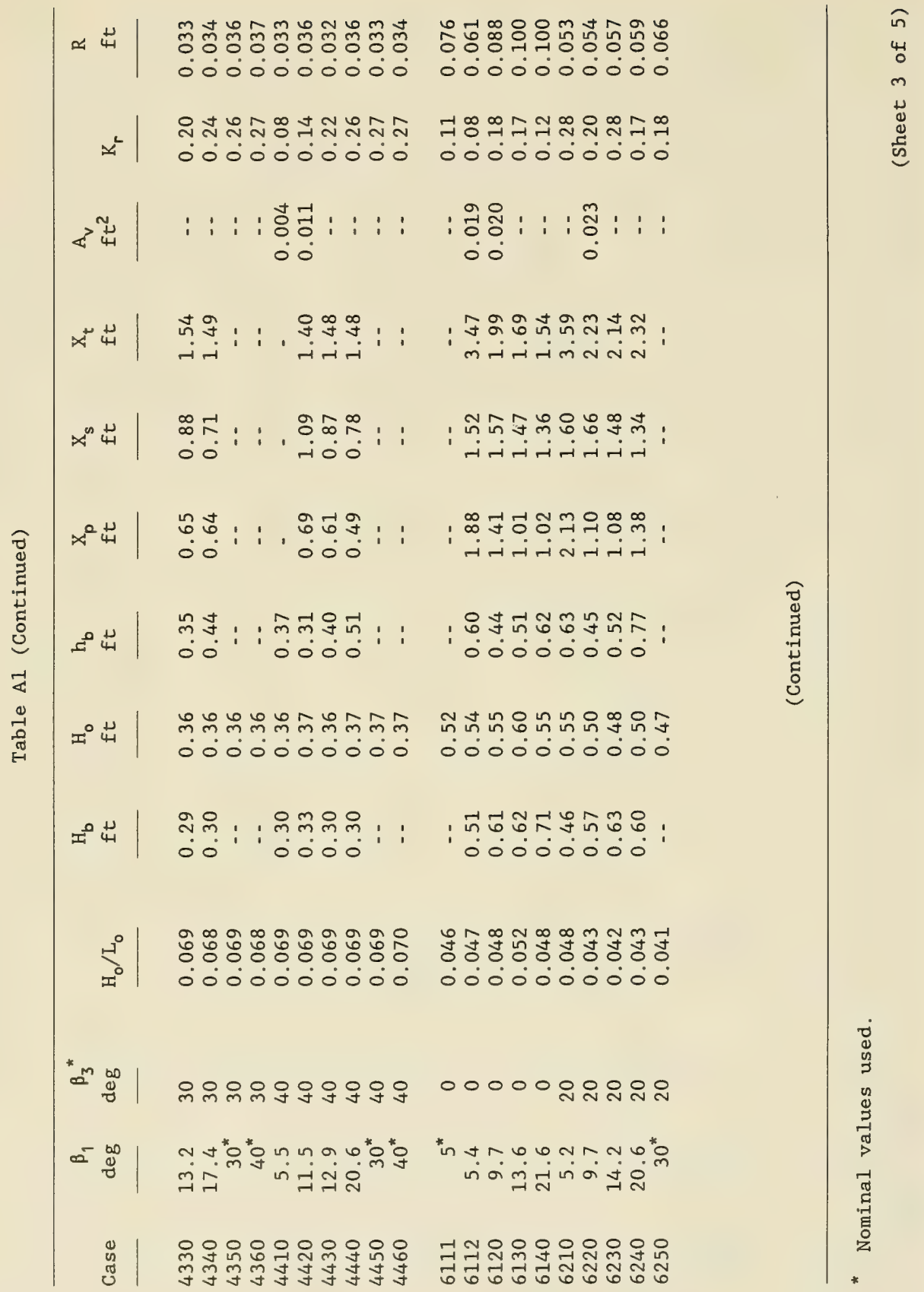




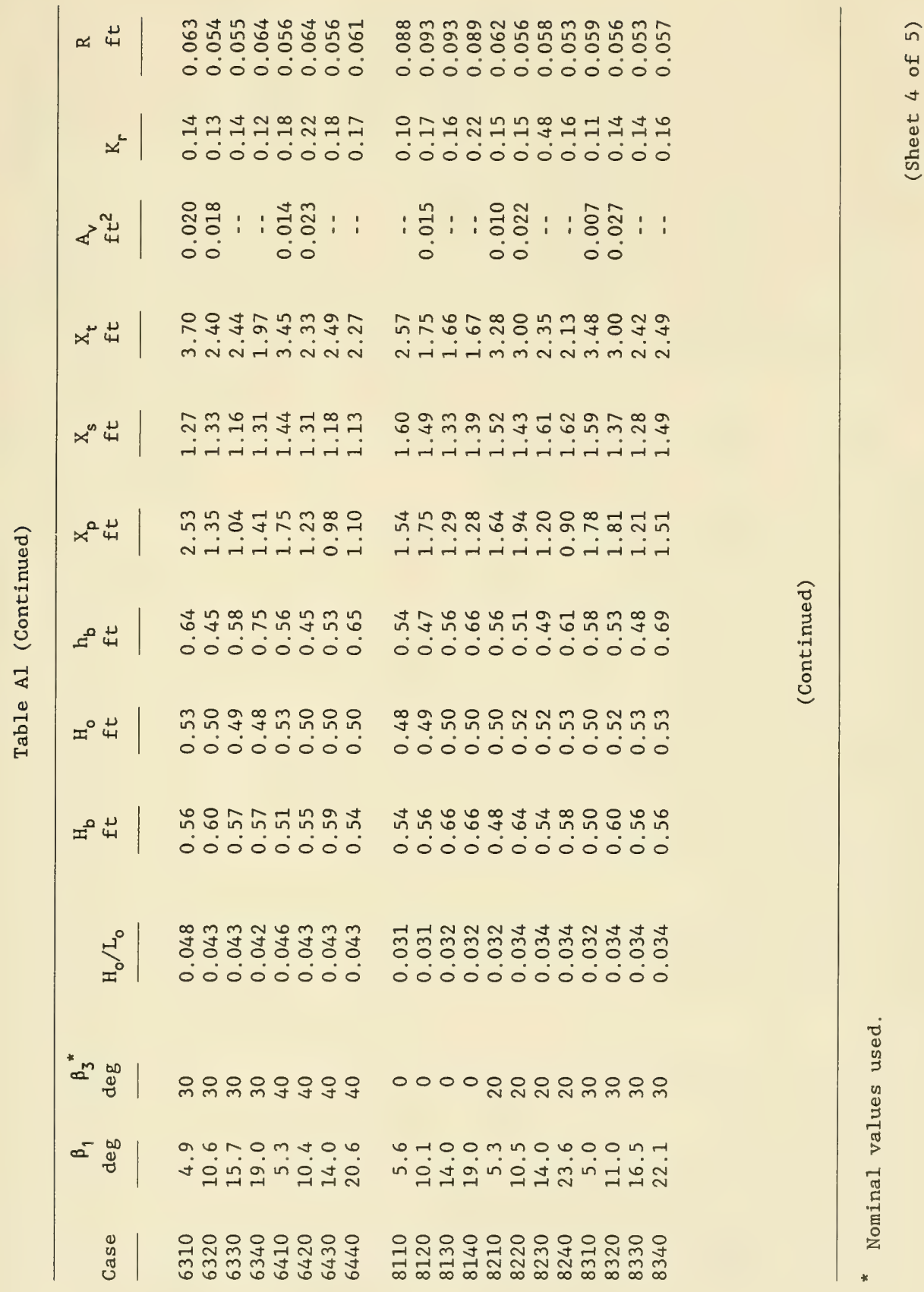




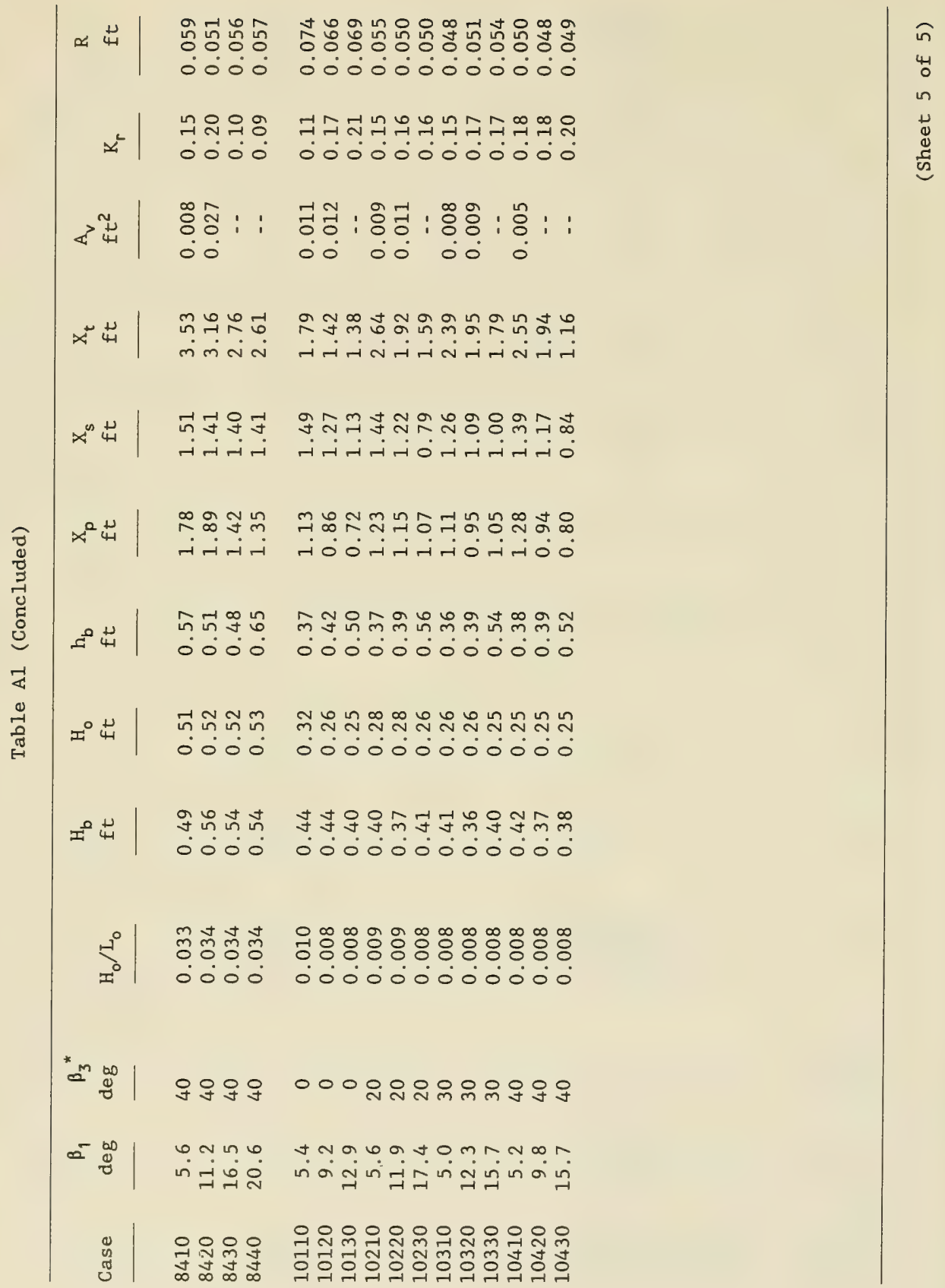


Table A2

Summary of Plane-Slope Tests

\begin{tabular}{|c|c|c|c|c|c|c|c|c|}
\hline Case & $\mathrm{m}$ & $\begin{array}{c}\mathrm{T} \\
\mathrm{sec}\end{array}$ & $\begin{array}{l}\mathrm{L}_{\circ} \\
\mathrm{ft}\end{array}$ & $\begin{array}{l}\mathrm{H}_{\mathrm{o}} \\
\mathrm{ft}\end{array}$ & $\mathrm{H}_{\mathrm{o}} / \mathrm{L}_{\mathrm{o}}$ & $\begin{array}{l}\mathrm{H}_{\mathrm{b}} \\
\mathrm{ft}\end{array}$ & $\begin{array}{l}h_{b} \\
\text { ft }\end{array}$ & $\begin{array}{l}x_{p} \\
f t\end{array}$ \\
\hline 2000 & 0.033 & 1.02 & 5.29 & 0.49 & 0.092 & 0.40 & 0.67 & 2.86 \\
\hline 4000 & 0.033 & 1.02 & 5.31 & 0.35 & 0.066 & 0.27 & 0.43 & 1.84 \\
\hline 6000 & 0.033 & 1.49 & 11.35 & 0.52 & 0.046 & 0.51 & 0.71 & 3.55 \\
\hline 8000 & 0.033 & 1.74 & 15.43 & 0.47 & 0.030 & 0.54 & 0.71 & 2.94 \\
\hline 10000 & 0.033 & 2.49 & 31.65 & 0.28 & 0.009 & 0.42 & 0.49 & 2.15 \\
\hline
\end{tabular}


Table A3

Results of Iversen (1952)*

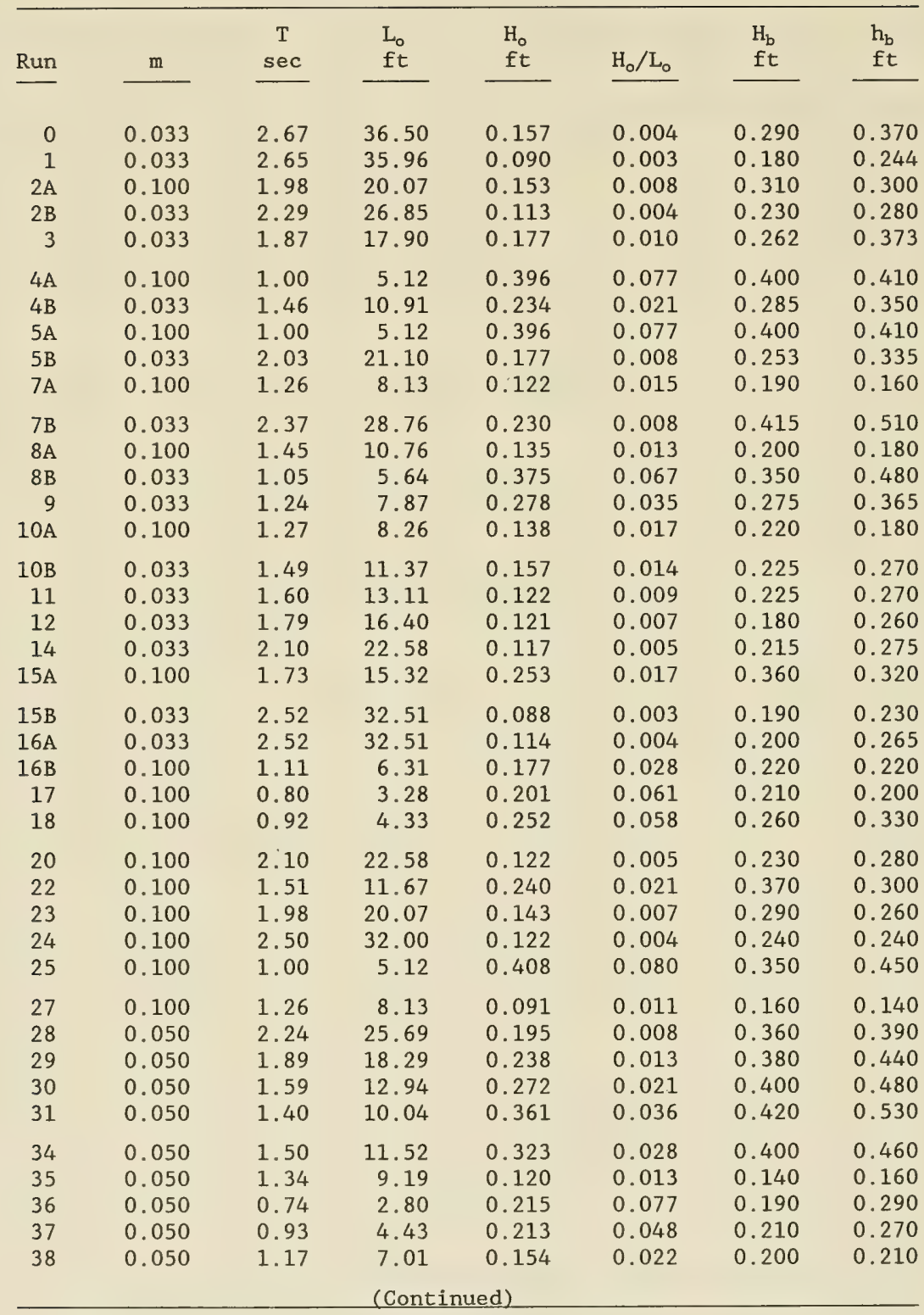

* See References at the end of the main text. 
Table A3 (Concluded)

\begin{tabular}{|c|c|c|c|c|c|c|c|}
\hline Run & $\mathrm{m}$ & $\begin{array}{c}\mathrm{T} \\
\mathrm{sec}\end{array}$ & $\begin{array}{l}\mathrm{L}_{\mathrm{o}} \\
\mathrm{ft}\end{array}$ & $\begin{array}{l}\mathrm{H}_{\circ} \\
\mathrm{ft}\end{array}$ & $\mathrm{H}_{\mathrm{o}} / \mathrm{L}_{\mathrm{o}}$ & $\begin{array}{l}\mathrm{H}_{\mathrm{b}} \\
\mathrm{ft}\end{array}$ & $\begin{array}{l}h_{b} \\
f t\end{array}$ \\
\hline 39 & 0.050 & 1.12 & 6.42 & 0.173 & 0.027 & 0.190 & 0.230 \\
\hline 40 & 0.050 & 1.03 & 5.43 & 0.196 & 0.036 & 0.180 & 0.250 \\
\hline 41 & 0.050 & 1.55 & 12.30 & 0.102 & 0.008 & 0.450 & 0.180 \\
\hline 42 & 0.050 & 1.26 & 8.13 & 0.284 & 0.035 & 0.330 & 0.340 \\
\hline 43 & 0.050 & 1.41 & 10.18 & 0.224 & 0.022 & 0.270 & 0.230 \\
\hline 44 & 0.050 & 1.33 & 9.06 & 0.263 & 0.029 & 0.300 & 0.340 \\
\hline 45 & 0.050 & 1.15 & 6.77 & 0.325 & 0.048 & 0.310 & 0.390 \\
\hline 46 & 0.050 & 1.04 & 5.54 & 0.404 & 0.073 & 0.350 & 0.540 \\
\hline 47 & 0.050 & 1.67 & 14.28 & 0.186 & 0.013 & 0.270 & 0.290 \\
\hline 48 & 0.050 & 1.93 & 19.07 & 0.151 & 0.008 & 0.250 & 0.250 \\
\hline 58 & 0.020 & 1.00 & 5.12 & 0.368 & 0.072 & 0.303 & 0.403 \\
\hline 59 & 0.020 & 1.00 & 5.12 & 0.243 & 0.047 & 0.218 & - \\
\hline 60 & 0.020 & 1.00 & 5.12 & 0.193 & 0.038 & 0.185 & - \\
\hline 61 & 0.020 & 0.90 & 4.15 & 0.293 & 0.071 & 0.222 & 0.326 \\
\hline 62 & 0.020 & 0.81 & 3.36 & 0.305 & 0.091 & 0.250 & - \\
\hline 63 & 0.020 & 1.17 & 7.01 & 0.264 & 0.038 & 0.274 & 0.321 \\
\hline 66 & 0.020 & 1.74 & 15.50 & 0.202 & 0.013 & 0.283 & 0.334 \\
\hline 68 & 0.020 & 1.62 & 13.44 & 0.255 & 0.019 & 0.268 & 0.306 \\
\hline 70 & 0.020 & 1.13 & 6.54 & 0.304 & 0.047 & 0.297 & 0.350 \\
\hline 71 & 0.020 & 2.00 & 20.48 & 0.188 & 0.009 & 0.208 & 0.222 \\
\hline 73 & 0.020 & 1.30 & 8.65 & 0.264 & 0.031 & 0.248 & 0.328 \\
\hline 74 & 0.020 & 0.95 & 4.62 & 0.233 & 0.050 & 0.191 & 0.228 \\
\hline 77 & 0.020 & 1.35 & 9.33 & 0.208 & 0.022 & 0.199 & 0.231 \\
\hline 78 & 0.020 & 2.43 & 30.23 & 0.224 & 0.007 & 0.353 & - \\
\hline 80 & 0.020 & 2.25 & 25.92 & 0.168 & 0.007 & 0.217 & - \\
\hline 81 & 0.020 & 2.65 & 35.96 & 0.234 & 0.007 & 0.398 & 0.513 \\
\hline 82 & 0.020 & 2.65 & 35.96 & 0.176 & 0.005 & 0.320 & 0.422 \\
\hline 83 & 0.020 & 1.90 & 18.48 & 0.137 & 0.007 & 0.181 & 0.212 \\
\hline
\end{tabular}


Table A4

Results of Horikawa and Kuo (1967)

\begin{tabular}{|c|c|c|c|c|c|c|c|}
\hline Run & $\mathrm{m}$ & $\begin{array}{c}\mathrm{T} \\
\mathrm{sec} \\
\end{array}$ & $\begin{array}{l}\mathrm{L}_{\circ} \\
\mathrm{cm}\end{array}$ & $\begin{array}{l}\mathrm{H}_{\mathrm{o}} \\
\mathrm{cm}\end{array}$ & $\mathrm{Ho}_{\mathrm{o}} / \mathrm{L}_{\mathrm{o}}$ & $\begin{array}{l}\mathrm{H}_{\mathrm{b}} \\
\mathrm{cm}\end{array}$ & $\begin{array}{l}\mathrm{h}_{\mathrm{b}} \\
\mathrm{cm}\end{array}$ \\
\hline 1 & 0.0125 & 1.2 & 224.6 & 8.8 & 0.039 & 8.6 & 12.5 \\
\hline 2 & 0.0125 & 1.2 & 224.6 & 9.1 & 0.041 & 9.1 & 12.5 \\
\hline 3 & 0.0125 & 1.2 & 224.6 & 12.0 & 0.053 & 12.2 & 13.8 \\
\hline 4 & 0.0125 & 1.2 & 224.6 & 14.8 & 0.066 & 14.2 & 16.3 \\
\hline 5 & 0.0125 & 1.2 & 224.6 & 11.7 & 0.052 & 11.6 & 16.3 \\
\hline 6 & 0.0125 & 1.2 & 224.6 & 13.0 & 0.058 & 13.1 & 16.3 \\
\hline 7 & 0.0125 & 1.2 & 224.6 & 13.3 & 0.059 & 13.2 & 17.5 \\
\hline 8 & 0.0125 & 1.2 & 224.6 & 13.5 & 0.060 & 13.3 & 20.0 \\
\hline 9 & 0.0125 & 1.2 & 224.6 & 14.6 & 0.065 & 14.7 & 22.5 \\
\hline 10 & 0.0125 & 1.2 & 224.6 & 14.2 & 0.063 & 13.9 & 21.3 \\
\hline 11 & 0.0125 & 1.2 & 224.6 & 11.8 & 0.053 & 18.2 & 21.3 \\
\hline 12 & 0.0125 & 1.2 & 224.6 & 16.4 & 0.073 & 16.3 & 26.3 \\
\hline 13 & 0.0125 & 1.4 & 305.8 & 8.0 & 0.026 & 7.8 & 12.5 \\
\hline 14 & 0.0125 & 1.4 & 305.8 & 9.0 & 0.029 & 9.3 & 13.8 \\
\hline 15 & 0.0125 & 1.4 & 305.8 & 9.7 & 0.032 & 9.4 & 15.0 \\
\hline 16 & 0.0125 & 1.4 & 305.8 & 11.6 & 0.038 & 11.6 & 15.0 \\
\hline 17 & 0.0125 & 1.4 & 305.8 & 11.4 & 0.037 & 11.5 & 16.3 \\
\hline 18 & 0.0125 & 1.4 & 305.8 & 12.9 & 0.042 & 12.3 & 18.8 \\
\hline 19 & 0.0125 & 1.4 & 305.8 & 13.5 & 0.044 & 13.0 & 20.0 \\
\hline 20 & 0.0125 & 1.4 & 305.8 & 14.1 & 0.046 & 13.5 & 21.3 \\
\hline 21 & 0.0125 & 1.4 & 305.8 & 14.8 & 0.048 & 13.8 & 21.3 \\
\hline 22 & 0.0125 & 1.4 & 305.8 & 15.3 & 0.050 & 16.4 & 22.5 \\
\hline 23 & 0.0125 & 1.4 & 305.8 & 16.7 & 0.055 & 17.3 & 25.0 \\
\hline 24 & 0.0125 & 1.6 & 399.4 & 7.9 & 0.020 & 7.9 & 11.3 \\
\hline 25 & 0.0125 & 1.6 & 399.4 & 9.8 & 0.025 & 10.2 & 12.5 \\
\hline 26 & 0.0125 & 1.6 & 399.4 & 10.5 & 0.026 & 10.3 & 15.0 \\
\hline 27 & 0.0125 & 1.6 & 399.4 & 11.1 & 0.028 & 11.7 & 16.3 \\
\hline 28 & 0.0125 & 1.6 & 399.4 & 12.4 & 0.031 & 13.7 & 16.3 \\
\hline 29 & 0.0125 & 1.6 & 399.4 & 13.1 & 0.033 & 14.3 & 16.3 \\
\hline 30 & 0.0125 & 1.6 & 399.4 & 14.2 & 0.036 & 15.2 & 17.5 \\
\hline 31 & 0.0125 & 1.6 & 399.4 & 15.9 & 0.040 & 14.7 & $21: 3$ \\
\hline 32 & 0.0125 & 1.6 & 399.4 & 16.8 & 0.042 & 15.0 & 22.5 \\
\hline 33 & 0.0125 & 1.8 & 505.4 & 7.4 & 0.015 & 8.8 & 11.3 \\
\hline 34 & 0.0125 & 1.8 & 505.4 & 8.3 & 0.016 & 9.2 & 12.5 \\
\hline 35 & 0.0125 & 1.8 & 505.4 & 9.0 & 0.018 & 9.4 & 15.0 \\
\hline 36 & 0.0125 & 1.8 & 505.4 & 9.6 & 0.019 & 10.0 & 13.8 \\
\hline 37 & 0.0125 & 1.8 & 505.4 & 10.5 & 0.021 & 10.8 & 15.0 \\
\hline 38 & 0.0125 & 1.8 & 505.4 & 10.9 & 0.022 & 11.6 & 16.3 \\
\hline 39 & 0.0125 & 1.8 & 505.4 & 11.3 & 0.022 & 11.8 & 16.3 \\
\hline 40 & 0.0125 & 1.8 & 505.4 & 11.8 & 0.023 & 11.5 & 18.8 \\
\hline
\end{tabular}

(Continued) 
Table A4 (Continued)

\begin{tabular}{|c|c|c|c|c|c|c|c|}
\hline Run & $\mathrm{m}$ & $\begin{array}{c}\mathrm{T} \\
\mathrm{sec} \\
\end{array}$ & $\begin{array}{l}\mathrm{L}_{\circ} \\
\mathrm{cm}\end{array}$ & $\begin{array}{l}\mathrm{H}_{\mathrm{o}} \\
\mathrm{cm}\end{array}$ & $\mathrm{H}_{\mathrm{o}} / \mathrm{L}_{\mathrm{o}}$ & $\begin{array}{l}\mathrm{H}_{\mathrm{b}} \\
\mathrm{cm}\end{array}$ & $\begin{array}{l}\mathrm{h}_{\mathrm{b}} \\
\mathrm{cm}\end{array}$ \\
\hline 41 & 0.0125 & 1.8 & 505.4 & 13.1 & 0.026 & 12.4 & 18.8 \\
\hline 42 & 0.0125 & 1.8 & 505.4 & 13.9 & 0.027 & 12.6 & 20.0 \\
\hline 43 & 0.0125 & 1.8 & 505.4 & 14.5 & 0.029 & 13.8 & 21.3 \\
\hline 44 & 0.0125 & 1.8 & 505.4 & 15.3 & 0.030 & 13.8 & 21.3 \\
\hline 45 & 0.0125 & 1.8 & 505.4 & 15.4 & 0.030 & 15.0 & 22.5 \\
\hline 46 & 0.0125 & 2.0 & 624.0 & 8.5 & 0.014 & 8.3 & 13.1 \\
\hline 47 & 0.0125 & 2.0 & 624.0 & 8.9 & 0.014 & 9.1 & 13.8 \\
\hline 48 & 0.0125 & 2.0 & 624.0 & 9.8 & 0.016 & 10.4 & 15.0 \\
\hline 49 & 0.0125 & 2.0 & 624.0 & 10.7 & 0.017 & 11.2 & 15.0 \\
\hline 50 & 0.0125 & 2.0 & 624.0 & 11.3 & 0.018 & 11.8 & 15.0 \\
\hline 51 & 0.0125 & 2.0 & 624.0 & 11.5 & 0.018 & 12.0 & 16.8 \\
\hline 52 & 0.0125 & 2.0 & 624.0 & 12.0 & 0.019 & 11.9 & 16.5 \\
\hline 53 & 0.0125 & 2.0 & 624.0 & 12.6 & 0.020 & 12.5 & 20.0 \\
\hline 54 & 0.0125 & 2.0 & 624.0 & 13.3 & 0.021 & 13.6 & 20.0 \\
\hline 55 & 0.0125 & 2.0 & 624.0 & 14.4 & 0.023 & 14.6 & 21.3 \\
\hline 56 & 0.0125 & 2.0 & 624.0 & 15.2 & 0.024 & 15.4 & 21.3 \\
\hline 57 & 0.0125 & 2.0 & 624.0 & 16.3 & 0.026 & 15.5 & 25.0 \\
\hline 74 & 0.0333 & 2.2 & 755.0 & 13.0 & 0.017 & 12.8 & 17.3 \\
\hline 75 & 0.0333 & 2.2 & 755.0 & 12.1 & 0.016 & 10.0 & 14.5 \\
\hline 76 & 0.0333 & 2.2 & 755.0 & 10.4 & 0.014 & 11.5 & 15.2 \\
\hline 77 & 0.0333 & 2.2 & 755.0 & 10.8 & 0.014 & 11.2 & 13.5 \\
\hline 78 & 0.0333 & 2.2 & 755.0 & 9.5 & 0.013 & 7.6 & 11.8 \\
\hline 79 & 0.0333 & 2.2 & 755.0 & 8.6 & 0.011 & 11.3 & 13.5 \\
\hline 80 & 0.0333 & 2.2 & 755.0 & 8.5 & 0.011 & 10.8 & 13.5 \\
\hline 81 & 0.0333 & 2.2 & 755.0 & 6.8 & 0.009 & 8.7 & 11.8 \\
\hline 82 & 0.0333 & 2.2 & 755.0 & 6.3 & 0.008 & 9.2 & 10.8 \\
\hline 83 & 0.0333 & 2.2 & 755.0 & 5.6 & 0.007 & 8.4 & 9.8 \\
\hline 84 & 0.0333 & 2.2 & 755.0 & 4.7 & 0.006 & 6.0 & 6.8 \\
\hline 85 & 0.0333 & 1.4 & 305.8 & 16.1 & 0.053 & 16.7 & 20.2 \\
\hline 86 & 0.0333 & 1.4 & 305.8 & 14.6 & 0.048 & 15.5 & 18.4 \\
\hline 87 & 0.0333 & 1.4 & 305.8 & 14.0 & 0.046 & 15.1 & 18.3 \\
\hline 88 & 0.0333 & 1.4 & 305.8 & 13.3 & 0.043 & 12.7 & 18.2 \\
\hline 89 & 0.0333 & 1.4 & 305.8 & 11.7 & 0.038 & 12.1 & 18.2 \\
\hline 92 & 0.0333 & 1.4 & 305.8 & 6.9 & 0.023 & 6.3 & 7.5 \\
\hline 93 & 0.0500 & 1.4 & 305.8 & 8.2 & 0.027 & 7.5 & 6.0 \\
\hline 94 & 0.0500 & 1.4 & 305.8 & 9.2 & 0.030 & 9.4 & 6.0 \\
\hline 95 & 0.0500 & 1.4 & 305.8 & 10.5 & 0.034 & 10.3 & 8.0 \\
\hline 96 & 0.0500 & 1.4 & 305.8 & 12.0 & 0.039 & 11.1 & 8.0 \\
\hline 97 & 0.0500 & 1.4 & 305.8 & 11.1 & 0.036 & 11.7 & 14.0 \\
\hline 90 & 0.0333 & 1.4 & 305.8 & 10.0 & 0.033 & 10.4 & 15.8 \\
\hline
\end{tabular}

(Continued)

(Sheet 2 of 3 ) 
Table A4 (Concluded)

\begin{tabular}{|c|c|c|c|c|c|c|c|}
\hline Run & m & $\begin{array}{c}\mathrm{T} \\
\mathrm{sec}\end{array}$ & $\begin{array}{l}\mathrm{L}_{\mathrm{o}} \\
\mathrm{cm}\end{array}$ & $\begin{array}{l}\mathrm{H}_{\circ} \\
\mathrm{cm}\end{array}$ & $\mathrm{H}_{\mathrm{o}} / \mathrm{L}_{\mathrm{o}}$ & $\begin{array}{l}\mathrm{H}_{\mathrm{b}} \\
\mathrm{cm}\end{array}$ & $\begin{array}{l}h_{b} \\
\mathrm{~cm}\end{array}$ \\
\hline 91 & 0.0333 & 1.4 & 305.8 & 8.2 & 0.027 & 6.9 & 7.5 \\
\hline 98 & 0.0500 & 1.4 & 305.8 & 13.2 & 0.043 & 13.9 & 14.0 \\
\hline 99 & 0.0500 & 1.4 & 305.8 & 14.2 & 0.046 & 13.1 & 16.0 \\
\hline 100 & 0.0500 & 1.4 & 305.8 & 16.1 & 0.053 & 13.2 & 16.0 \\
\hline 101 & 0.0500 & 1.4 & 305.8 & 17.3 & 0.057 & 15.7 & 20.5 \\
\hline 102 & 0.0500 & 1.4 & 305.8 & 16.9 & 0.055 & 16.6 & 20.5 \\
\hline 103 & 0.0500 & 2.2 & 755.0 & 5.6 & 0.007 & 6.5 & 7.3 \\
\hline 104 & 0.0500 & 2.2 & 755.0 & 6.3 & 0.008 & 7.8 & 10.8 \\
\hline 105 & 0.0500 & 2.2 & 755.0 & 7.0 & 0.009 & 8.3 & 9.3 \\
\hline 105 & 0.0500 & 2.2 & 755.0 & 8.3 & 0.011 & 10.5 & 9.3 \\
\hline 107 & 0.0500 & 2.2 & 755.0 & 9.5 & 0.013 & 12.3 & 10.5 \\
\hline 108 & 0.0500 & 2.2 & 755.0 & 9.8 & 0.013 & 11.3 & 12.3 \\
\hline 109 & 0.0500 & 2.2 & 755.0 & 10.1 & 0.013 & 11.6 & 12.8 \\
\hline 110 & 0.0500 & 2.2 & 755.0 & 10.6 & 0.014 & 11.7 & 13.5 \\
\hline 111 & 0.0500 & 2.2 & 755.0 & 11.3 & 0.015 & 12.3 & 13.3 \\
\hline 112 & 0.0500 & 2.2 & 755.0 & 12.1 & 0.016 & 12.8 & 15.3 \\
\hline 113 & 0.0500 & 2.3 & 825.2 & 13.7 & 0.017 & 15.6 & 16.5 \\
\hline
\end{tabular}


Table A5

Results of Galvin (1969)

\begin{tabular}{|c|c|c|c|c|c|c|c|c|}
\hline Run & m & $\begin{array}{c}\mathrm{T} \\
\mathrm{sec}\end{array}$ & $\begin{array}{l}\mathrm{L}_{\circ} \\
\mathrm{ft}\end{array}$ & $\begin{array}{l}\mathrm{H}_{\circ} \\
\mathrm{ft}\end{array}$ & $\mathrm{H}_{\mathrm{o}} / \mathrm{L}_{\mathrm{o}}$ & $\begin{array}{l}\mathrm{H}_{\mathrm{b}} \\
\mathrm{ft}\end{array}$ & $\begin{array}{l}\mathrm{h}_{\mathrm{b}} \\
\mathrm{ft}\end{array}$ & $\begin{array}{l}x_{p} \\
f t\end{array}$ \\
\hline 1 & 0.05 & 2.0 & 20.5 & 0.18 & 0.0089 & 0.31 & 0.34 & 0.88 \\
\hline 2 & 0.05 & 4.0 & 82.0 & 0.13 & 0.0016 & 0.37 & 0.33 & 1.18 \\
\hline 3 & 0.05 & 5.0 & 128.1 & 0.12 & 0.0009 & 0.39 & 0.36 & 1.45 \\
\hline 4 & 0.05 & 4.0 & 82.0 & 0.23 & 0.0028 & 0.58 & 0.53 & 2.11 \\
\hline 5 & 0.05 & 5.0 & 128.1 & 0.17 & 0.0013 & 0.52 & 0.48 & 2.10 \\
\hline 6 & 0.05 & 6.0 & 184.5 & 0.13 & 0.0007 & 0.45 & 0.44 & 1.77 \\
\hline 7 & 0.05 & 6.0 & 184.5 & - & - - & 0.46 & 0.60 & 2.24 \\
\hline 8 & 0.10 & 1.0 & 5.1 & 0.19 & 0.0378 & 0.21 & 0.20 & 0.69 \\
\hline 9 & 0.10 & 2.0 & 20.5 & - - & -. & 0.13 & 0.13 & 0.27 \\
\hline 10 & 0.10 & 5.0 & 128.1 & -- & - - & 0.47 & 0.29 & 1.67 \\
\hline 11 & 0.10 & 6.0 & 184.5 & 0.15 & 0.0008 & 0.33 & 0.25 & 1.20 \\
\hline 12 & 0.10 & 1.0 & 5.1 & 0.23 & 0.0448 & 0.24 & 0.20 & 0.64 \\
\hline 13 & 0.10 & 2.0 & 20.5 & 0.09 & 0.0045 & 0.14 & 0.13 & 0.33 \\
\hline 14 & 0.10 & 2.0 & 20.5 & 0.27 & 0.0133 & 0.39 & 0.30 & 0.94 \\
\hline 15 & 0.10 & 5.0 & 128.1 & 0.23 & 0.0018 & 0.49 & 0.33 & 1.37 \\
\hline 16 & 0.10 & 2.0 & 20.5 & 0.11 & 0.0052 & 0.15 & 0.15 & 0.36 \\
\hline 17 & 0.10 & 2.0 & 20.5 & 0.32 & 0.0155 & 0.31 & 0.38 & 1.21 \\
\hline 18 & 0.10 & 4.0 & 82.0 & 0.23 & 0.0028 & 0.48 & 0.34 & 1.48 \\
\hline 19 & 0.20 & 1.0 & 5.1 & 0.19 & 0.0378 & 0.20 & 0.20 & 0.44 \\
\hline 20 & 0.20 & 1.0 & 5.1 & 0.23 & 0.0448 & 0.30 & 0.26 & 0.72 \\
\hline 21 & 0.20 & 1.0 & 5.1 & 0.26 & 0.0503 & 0.30 & 0.27 & 0.81 \\
\hline 22 & 0.20 & 2.0 & 20.5 & 0.11 & 0.0052 & 0.23 & 0.21 & 0.34 \\
\hline
\end{tabular}

Table A6

Results of Saeki and Sasaki (1973)

\begin{tabular}{|c|c|c|c|c|c|c|c|}
\hline Run & $\mathrm{m}$ & $\begin{array}{c}\mathrm{T} \\
\text { sec }\end{array}$ & $\begin{array}{l}\mathrm{L}_{\circ} \\
\mathrm{cm}\end{array}$ & $\begin{array}{l}\mathrm{H}_{\circ} \\
\mathrm{cm}\end{array}$ & $\mathrm{H}_{\mathrm{o}} / \mathrm{L}_{\mathrm{o}}$ & $\begin{array}{l}\mathrm{H}_{\mathrm{b}} \\
\mathrm{cm}\end{array}$ & $\begin{array}{l}\mathrm{h}_{\mathrm{b}} \\
\mathrm{cm}\end{array}$ \\
\hline 1 & 0.02 & 1.3 & 263.6 & 10.3 & 0.039 & 10.6 & 16.4 \\
\hline 2 & 0.02 & 2.5 & 975.0 & 5.3 & 0.005 & 9.9 & 9.7 \\
\hline
\end{tabular}


Table A7

Results of Iwagaki et al. (1974)

\begin{tabular}{|c|c|c|c|c|c|c|c|}
\hline Run & m & $\begin{array}{c}\mathrm{T} \\
\mathrm{sec}\end{array}$ & $\begin{array}{l}\mathrm{L}_{\circ} \\
\mathrm{cm}\end{array}$ & $\begin{array}{l}\mathrm{H}_{\mathrm{o}} \\
\mathrm{cm}\end{array}$ & $\mathrm{H}_{\mathrm{o}} / \mathrm{L}_{\mathrm{o}}$ & $\begin{array}{l}\mathrm{H}_{\mathrm{b}} \\
\mathrm{cm}\end{array}$ & $\begin{array}{l}\mathrm{h}_{\mathrm{b}} \\
\mathrm{cm}\end{array}$ \\
\hline 1 & 0.10 & 1.0 & 156.1 & 9.1 & 0.058 & 9.7 & 11.1 \\
\hline 2 & 0.10 & 1.0 & 156.1 & 6.6 & 0.042 & 6.8 & 7.5 \\
\hline 3 & 0.10 & 1.0 & 156.1 & 4.4 & 0.028 & 5.1 & 6.1 \\
\hline 4 & 0.10 & 1.5 & 351.3 & 8.1 & 0.023 & 10.1 & 12.0 \\
\hline 5 & 0.10 & 1.5 & 351.3 & 6.7 & 0.019 & 9.9 & 9.8 \\
\hline 6 & 0.10 & 1.5 & 351.3 & 4.6 & 0.013 & 6.8 & 6.7 \\
\hline 7 & 0.05 & 1.0 & 156.1 & 11.4 & 0.073 & 10.9 & 15.8 \\
\hline 8 & 0.05 & 1.0 & 156.1 & 8.0 & 0.051 & 8.4 & 10.6 \\
\hline 9 & 0.05 & 1.0 & 156.1 & 4.8 & 0.031 & 5.7 & 6.8 \\
\hline 10 & 0.05 & 1.5 & 351.3 & 11.2 & 0.032 & 12.8 & 14.8 \\
\hline 11 & 0.05 & 1.5 & 351.3 & 6.7 & 0.019 & 8.3 & 10.5 \\
\hline 12 & 0.05 & 1.5 & 351.3 & 3.5 & 0.010 & 6.2 & 6.0 \\
\hline 13 & 0.05 & 2.0 & 624.5 & 6.9 & 0.011 & 9.2 & 12.0 \\
\hline 14 & 0.05 & 2.0 & 624.5 & 5.0 & 0.008 & 8.0 & 9.7 \\
\hline 15 & 0.05 & 2.0 & 624.5 & 3.1 & 0.005 & 5.3 & 6.3 \\
\hline 16 & 0.03 & 1.0 & 156.1 & 8.0 & 0.051 & 8.1 & 11.8 \\
\hline 17 & 0.03 & 1.0 & 156.1 & 6.1 & 0.039 & 6.6 & 9.7 \\
\hline 18 & 0.03 & 1.0 & 156.1 & 4.1 & 0.026 & 4.4 & 6.7 \\
\hline 19 & 0.03 & 1.5 & 351.3 & 8.8 & 0.025 & 10.9 & 12.8 \\
\hline 20 & 0.03 & 1.5 & 351.3 & 5.6 & 0.016 & 7.5 & 9.9 \\
\hline 21 & 0.03 & 2.0 & 624.5 & 6.9 & 0.011 & 9.6 & 12.3 \\
\hline 22 & 0.03 & 2.0 & 624.5 & 5.0 & 0.008 & 8.3 & 9.9 \\
\hline 23 & 0.03 & 2.0 & 624.5 & 3.1 & 0.005 & 5.9 & 6.9 \\
\hline
\end{tabular}


Table A8

Results of Walker (1974b)

\begin{tabular}{|c|c|c|c|c|c|c|c|}
\hline Run & m & $\begin{array}{c}\mathrm{T} \\
\text { sec } \\
\end{array}$ & $\begin{array}{l}\mathrm{L}_{\mathrm{o}} \\
\mathrm{ft}\end{array}$ & $\begin{array}{l}\mathrm{H}_{\circ} \\
\mathrm{ft}\end{array}$ & $\mathrm{H}_{\mathrm{o}} / \mathrm{L}_{\mathrm{o}}$ & $\begin{array}{l}\mathrm{H}_{\mathrm{b}} \\
\mathrm{ft}\end{array}$ & $\begin{array}{l}h_{b} \\
f t \\
\end{array}$ \\
\hline 1 & 0.033 & 1.17 & 6.9 & 0.092 & 0.013 & 0.14 & 0.15 \\
\hline 2 & 0.033 & 1.67 & 14.2 & 0.062 & 0.004 & 0.12 & 0.13 \\
\hline 3 & 0.033 & 2.00 & 20.4 & 0.045 & 0.002 & 0.10 & 0.11 \\
\hline 4 & 0.033 & 2.33 & 27.8 & 0.034 & 0.001 & 0.08 & 0.10 \\
\hline 5 & 0.033 & 2.33 & 27.8 & 0.074 & 0.003 & 0.17 & 0.17 \\
\hline 6 & 0.033 & 2.00 & 20.4 & 0.097 & 0.005 & 0.19 & 0.18 \\
\hline 7 & 0.033 & 1.67 & 14.2 & 0.125 & 0.009 & 0.24 & 0.21 \\
\hline 8 & 0.033 & 1.17 & 6.9 & 0.180 & 0.026 & 0.25 & 0.20 \\
\hline 9 & 0.033 & 1.17 & 6.9 & 0.261 & 0.038 & 0.32 & 0.33 \\
\hline 10 & 0.033 & 1.67 & 14.2 & 0.185 & 0.013 & 0.31 & 0.30 \\
\hline 11 & 0.033 & 2.00 & 20.4 & 0.145 & 0.007 & 0.30 & 0.24 \\
\hline 12 & 0.033 & 2.33 & 27.8 & 0.112 & 0.004 & 0.25 & 0.22 \\
\hline 13 & 0.033 & 2.33 & 27.8 & 0.152 & 0.005 & 0.29 & 0.29 \\
\hline 14 & 0.033 & 2.00 & 20.4 & 0.200 & 0.010 & 0.34 & 0.30 \\
\hline 15 & 0.033 & 1.67 & 14.2 & 0.247 & 0.017 & 0.38 & 0.41 \\
\hline
\end{tabular}


Table A9

Results of Singamsetti and Wind (1980)

\begin{tabular}{|c|c|c|c|c|c|c|c|c|}
\hline Run & $\mathbf{m}$ & $\begin{array}{c}\mathrm{T} \\
\mathrm{sec}\end{array}$ & $\begin{array}{c}\mathrm{L}_{\circ} \\
\mathrm{m}\end{array}$ & $\begin{array}{c}\mathrm{H}_{\mathrm{o}} \\
\text { m }\end{array}$ & $\mathrm{H}_{\mathrm{o}} / \mathrm{L}_{\mathrm{o}}$ & $\begin{array}{c}\mathrm{H}_{\mathrm{b}} \\
\text { m }\end{array}$ & $\begin{array}{c}\mathrm{h}_{\mathrm{b}} \\
\mathrm{m}\end{array}$ & $\begin{array}{c}\mathrm{x}_{\mathrm{p}} \\
\mathrm{m}\end{array}$ \\
\hline A5-28 & 0.20 & 1.55 & 3.77 & 0.105 & 0.028 & 0.117 & 0.131 & 0.42 \\
\hline A5-39 & 0.20 & 1.55 & 3.76 & 0.149 & 0.040 & 0.193 & 0.160 & 0.61 \\
\hline A $5-40$ & 0.20 & 1.28 & 2.57 & 0.102 & 0.040 & 0.156 & 0.124 & 0.46 \\
\hline A5 - 27 & 0.20 & 1.28 & 2.57 & 0.071 & 0.027 & 0.097 & 0.103 & 0.34 \\
\hline A5- 18 & 0.20 & 1.55 & 3.76 & 0.067 & 0.018 & 0.095 & 0.078 & 0.32 \\
\hline A5-47 & 0.20 & 1.04 & 1.68 & 0.079 & 0.047 & 0.106 & 0.082 & 0.32 \\
\hline A5 - 48 & 0.20 & 1.28 & 2.57 & 0.125 & 0.048 & 0.160 & 0.134 & 0.57 \\
\hline A $5-43$ & 0.20 & 1.04 & 1.67 & 0.072 & 0.043 & 0.091 & 0.079 & 0.39 \\
\hline A5 - 60 & 0.20 & 1.04 & 1.68 & 0.101 & 0.060 & 0.117 & 0.099 & 0.36 \\
\hline A5-32 & 0.20 & 1.72 & 4.61 & 0.146 & 0.032 & 0.184 & 0.195 & 0.64 \\
\hline A5-21 & 0.20 & 1.72 & 4.62 & 0.097 & 0.021 & 0.125 & 0.117 & 0.41 \\
\hline A5 - 54 & 0.20 & 1.28 & 2.57 & 0.138 & 0.054 & 0.162 & 0.139 & 0.57 \\
\hline B5-41 & 0.20 & 1.28 & 2.57 & 0.105 & 0.041 & 0.121 & 0.104 & 0.46 \\
\hline B5 - 29 & 0.20 & 1. 28 & 2.57 & 0.076 & 0.029 & 0.089 & 0.083 & 0.34 \\
\hline B5 - 17 & 0.20 & 1.55 & 3.76 & 0.066 & 0.017 & 0.093 & 0.098 & 0.32 \\
\hline B5-49 & 0.20 & 1.04 & 1.68 & 0.084 & 0.050 & 0.087 & 0.082 & 0.32 \\
\hline B5 - 50 & 0.20 & 1.28 & 2.57 & 0.129 & 0.050 & 0.150 & 0.134 & 0.57 \\
\hline B5 - 42 & 0.20 & 1.04 & 1.68 & 0.071 & 0.042 & 0.077 & 0.099 & 0.39 \\
\hline B5 -60 & 0.20 & 1.04 & 1.68 & 0.102 & 0.060 & 0.118 & 0.099 & 0.36 \\
\hline B5- 31 & 0.20 & 1.72 & 4.63 & 0.142 & 0.031 & 0.184 & 0.195 & 0.64 \\
\hline B5-21 & 0.20 & 1.72 & 4.61 & 0.096 & 0.021 & 0.124 & 0.177 & 0.41 \\
\hline A10-29 & 0.10 & 1.55 & 3.75 & 0.108 & 0.029 & 0.137 & 0.129 & 0.70 \\
\hline A10-39 & 0.10 & 1.55 & 3.75 & 0.146 & 0.039 & 0.169 & 0.200 & 1.05 \\
\hline A10-37 & 0.10 & 1.28 & 2.56 & 0.095 & 0.037 & 0.118 & 0.129 & 0.68 \\
\hline A10-26 & 0.10 & 1.28 & 2.57 & 0.068 & 0.026 & 0.086 & 0.108 & 0.53 \\
\hline$A 10-20$ & 0.10 & 1.55 & 3.75 & 0.074 & 0.020 & 0.111 & 0.103 & 0.55 \\
\hline$A 10-45$ & 0.10 & 1.04 & 1.67 & 0.075 & 0.045 & 0.091 & 0.113 & 0.60 \\
\hline A10 - 47 & 0.10 & 1.28 & 2.55 & 0.120 & 0.047 & 0.135 & 0.146 & 0.83 \\
\hline A10-42 & 0.10 & 1.04 & 1.67 & 0.071 & 0.042 & 0.073 & 0.097 & 0.53 \\
\hline$A 10-62$ & 0.10 & 1.04 & 1.67 & 0.103 & 0.062 & 0.106 & 0.129 & 0.65 \\
\hline A10 - 28 & 0.10 & 1.72 & 4.62 & 0.132 & 0.029 & 0.169 & 0.186 & 0.93 \\
\hline A10-19 & 0.10 & 1.72 & 4.63 & 0.089 & 0.019 & 0.141 & 0.117 & $0: 62$ \\
\hline A10- 53 & 0.10 & 1.28 & 2.55 & 0.134 & 0.053 & 0.150 & 0.184 & 0.80 \\
\hline B10-28 & 0.10 & 1.55 & 3.75 & 0.109 & 0.029 & 0.141 & 0.130 & 0.60 \\
\hline B10-41 & 0.10 & 1.55 & 3.75 & 0.151 & 0.040 & 0.170 & 0.188 & 0.88 \\
\hline B10-40 & 0.10 & 1.28 & 2.56 & 0.103 & 0.040 & 0.118 & 0.131 & 0.55 \\
\hline B10-29 & 0.10 & 1.28 & 2.55 & 0.075 & 0.029 & 0.101 & 0.090 & 0.50 \\
\hline B10-20 & 0.10 & 1.55 & 3.75 & 0.077 & 0.020 & 0.119 & 0.108 & 0.55 \\
\hline B10-48 & 0.10 & 1.03 & 1.66 & 0.080 & 0.048 & 0.086 & 0.090 & 0.58 \\
\hline \multicolumn{9}{|c|}{ (Continued) } \\
\hline
\end{tabular}


Table A9 (Continued)

\begin{tabular}{|c|c|c|c|c|c|c|c|c|}
\hline Run & $\mathbf{m}$ & $\begin{array}{c}\mathrm{T} \\
\mathrm{sec}\end{array}$ & $\begin{array}{c}\mathrm{L}_{\mathrm{o}} \\
\mathrm{m}\end{array}$ & $\begin{array}{c}\mathrm{H}_{\mathrm{o}} \\
\mathrm{m}\end{array}$ & $\mathrm{H}_{\mathrm{o}} / \mathrm{L}_{\mathrm{o}}$ & $\begin{array}{c}\mathrm{H}_{\mathrm{b}} \\
\mathrm{m}\end{array}$ & $\begin{array}{c}h_{b} \\
\text { m }\end{array}$ & $\begin{array}{c}\mathrm{X}_{\mathrm{p}} \\
\mathrm{m}\end{array}$ \\
\hline B $10-50$ & 0.10 & 1.28 & 2.55 & 0.128 & 0.050 & 0.143 & 0.173 & 0.75 \\
\hline B10-42 & 0.10 & 1.03 & 1.66 & 0.070 & 0.042 & 0.078 & 0.078 & 0.43 \\
\hline B10-62 & 0.10 & 1.03 & 1.66 & 0.102 & 0.062 & 0.112 & 0.135 & 0.67 \\
\hline B10-30 & 0.10 & 1.71 & 4.57 & 0.137 & 0.030 & 0.175 & 0.185 & 0.90 \\
\hline B10-19 & 0.10 & 1.71 & 4.55 & 0.086 & 0.019 & 0.140 & 0.124 & 0.65 \\
\hline B10-55 & 0.10 & 1.28 & 2.55 & 0.141 & 0.055 & 0.156 & 0.188 & 0.90 \\
\hline A20-30 & 0.05 & 1.55 & 3.75 & 0.114 & 0.030 & 0.140 & 0.170 & 0.53 \\
\hline A20-41 & 0.05 & 1.55 & 3.75 & 0.156 & 0.042 & 0.174 & 0.202 & 0.75 \\
\hline A20-39 & 0.05 & 1.28 & 2.54 & 0.099 & 0.039 & 0.115 & 0.127 & 0.59 \\
\hline A20-32 & 0.05 & 1.28 & 2.55 & 0.081 & 0.032 & 0.097 & 0.102 & 0.60 \\
\hline A20-19 & 0.05 & 1.55 & 3.74 & 0.072 & 0.019 & 0.106 & 0.103 & 0.50 \\
\hline A20-52 & 0.05 & 1.04 & 1.68 & 0.088 & 0.052 & 0.088 & 0.108 & 0.66 \\
\hline A20-47 & 0.05 & 1.28 & 2.55 & 0.121 & 0.047 & 0.135 & 0.174 & 0.89 \\
\hline A20-42 & 0.05 & 1.04 & 1.68 & 0.070 & 0.042 & 0.079 & 0.093 & 0.45 \\
\hline A20-59 & 0.05 & 1.04 & 1.68 & 0.099 & 0.059 & 0.101 & 0.130 & 0.51 \\
\hline A20-29 & 0.05 & 1.73 & 4.65 & 0.135 & 0.029 & 0.176 & 0.202 & 0.89 \\
\hline A20-20 & 0.05 & 1.73 & 4.69 & 0.091 & 0.019 & 0.133 & 0.125 & 0.75 \\
\hline A20-62 & 0.05 & 1.28 & 2.55 & 0.158 & 0.062 & 0.163 & 0.203 & 0.77 \\
\hline B20-31 & 0.05 & 1.55 & 3.75 & 0.118 & 0.031 & 0.142 & 0.160 & 0.82 \\
\hline B20-42 & 0.05 & 1.55 & 3.75 & 0.158 & 0.042 & 0.181 & 0.213 & 0.78 \\
\hline B20-41 & 0.05 & 1.28 & 2.54 & 0.103 & 0.041 & 0.119 & 0.135 & 0.54 \\
\hline B20-33 & 0.05 & 1.28 & 2.55 & 0.084 & 0.033 & 0.101 & 0.106 & 0.58 \\
\hline B20-21 & 0.05 & 1.55 & 3.76 & 0.078 & 0.021 & 0.106 & 0.104 & 0.61 \\
\hline B20-53 & 0.05 & 1.04 & 1.68 & 0.089 & 0.053 & 0.092 & 0.100 & 0.59 \\
\hline B20-48 & 0.05 & 1.28 & 2.55 & 0.123 & 0.048 & 0.133 & 0.165 & 0.67 \\
\hline B20-44 & 0.05 & 1.04 & 1.69 & 0.074 & 0.044 & 0.077 & 0.083 & 0.47 \\
\hline B20-61 & 0.05 & 1.04 & 1.68 & 0.102 & 0.061 & 0.101 & 0.135 & 0.67 \\
\hline B20-29 & 0.05 & 1.73 & 4.67 & 0.136 & 0.029 & 0.171 & 0.181 & 0.81 \\
\hline B20-19 & 0.05 & 1.73 & 4.69 & 0.091 & 0.019 & 0.132 & 0.128 & 0.68 \\
\hline B20-63 & 0.05 & 1.28 & 2.55 & 0.160 & 0.063 & 0.165 & 0.193 & 0.58 \\
\hline A40-29 & 0.025 & 1.55 & 3.75 & 0.110 & 0.029 & 0.136 & 0.145 & 0.65 \\
\hline A40-39 & 0.025 & 1.55 & 3.74 & 0.146 & 0.039 & 0.170 & 0.203 & 1.25 \\
\hline A $40-40$ & 0.025 & 1.28 & 2.54 & 0.102 & 0.040 & 0.119 & 0.140 & 1.20 \\
\hline A $40-28$ & 0.025 & 1.28 & 2.54 & 0.072 & 0.028 & 0.093 & 0.111 & 0.80 \\
\hline$A 40-21$ & 0.025 & 1.55 & 3.76 & 0.080 & 0.021 & 0.112 & 0.118 & 0.57 \\
\hline A40-51 & 0.025 & 1.04 & 1.68 & 0.086 & 0.051 & 0.096 & 0.117 & -- \\
\hline A40-42 & 0.025 & 1.04 & 1.67 & 0.070 & 0.042 & 0.079 & 0.093 & 0.60 \\
\hline A40-59 & 0.025 & 1.28 & 2.54 & 0.151 & 0.059 & 0.159 & 0.220 & -- \\
\hline A $40-48$ & 0.025 & 1.28 & 2.55 & 0.122 & 0.048 & 0.137 & 0.151 & 0.60 \\
\hline
\end{tabular}


Table A9 (Concluded)

\begin{tabular}{|c|c|c|c|c|c|c|c|c|}
\hline Run & m & $\begin{array}{c}\mathrm{T} \\
\text { sec } \\
\end{array}$ & $\begin{array}{c}\mathrm{L}_{\mathrm{o}} \\
\mathrm{m}\end{array}$ & $\begin{array}{c}\mathrm{H}_{\mathrm{o}} \\
\mathrm{m}\end{array}$ & $\mathrm{H}_{\mathrm{o}} / \mathrm{L}_{\mathrm{o}}$ & $\begin{array}{c}\mathrm{H}_{\mathrm{b}} \\
\mathbf{m}\end{array}$ & $\begin{array}{c}\mathrm{h}_{\mathrm{b}} \\
\text { m }\end{array}$ & $\begin{array}{c}X_{p} \\
m\end{array}$ \\
\hline$A 40-62$ & 0.025 & 1.04 & 1.68 & 0.104 & 0.062 & 0.110 & 0.153 & -- \\
\hline A40-71 & 0.025 & 1.04 & 1.68 & 0.120 & 0.071 & 0.124 & 0.169 & 0.60 \\
\hline A40-80 & 0.025 & 1.04 & 1.68 & 0.134 & 0.080 & 0.133 & 0.205 & - - \\
\hline$A 40-20$ & 0.025 & 1.72 & 4.63 & 0.093 & 0.020 & 0.132 & 0.150 & 0.75 \\
\hline B $40-30$ & 0.025 & 1.55 & 3.75 & 0.111 & 0.030 & 0.136 & 0.145 & 0.65 \\
\hline$B 40-40$ & 0.025 & 1.55 & 3.76 & 0.150 & 0.040 & 0.169 & 0.213 & 1.25 \\
\hline B $40-41$ & 0.025 & 1.28 & 2.55 & 0.105 & 0.041 & 0.118 & 0.130 & 1.20 \\
\hline B $40-29$ & 0.025 & 1.28 & 2.54 & 0.073 & 0.029 & 0.092 & 0.101 & 0.70 \\
\hline B $40-22$ & 0.025 & 1.55 & 3.74 & 0.081 & 0.022 & 0.111 & 0.118 & 0.57 \\
\hline B $40-50$ & 0.025 & 1.04 & 1.68 & 0.084 & 0.050 & 0.095 & 0.127 & -. \\
\hline B $40-42$ & 0.025 & 1.04 & 1.69 & 0.071 & 0.042 & 0.078 & 0.093 & 0.60 \\
\hline B40-57 & 0.025 & 1.28 & 2.54 & 0.146 & 0.057 & 0.160 & 0.195 & -. \\
\hline B $40-47$ & 0.025 & 1.28 & 2.54 & 0.121 & 0.047 & 0.132 & 0.166 & 0.60 \\
\hline B40-61 & 0.025 & 1.04 & 1.68 & 0.103 & 0.061 & 0.109 & 0.143 & -. \\
\hline B $40-73$ & 0.025 & 1.04 & 1.68 & 0.123 & 0.073 & 0.125 & 0.164 & 0.60 \\
\hline B $40-79$ & 0.025 & 1.04 & 1.68 & 0.134 & 0.079 & 0.137 & 0.195 & - \\
\hline B $40-21$ & 0.025 & 1.72 & 4.61 & 0.095 & 0.021 & 0.140 & 0.155 & 0.57 \\
\hline
\end{tabular}


Table A10

Results of Mizuguchi (1981)

\begin{tabular}{|c|c|c|c|c|c|c|c|}
\hline Run & m & $\begin{array}{c}\mathrm{T} \\
\mathrm{sec}\end{array}$ & $\begin{array}{l}\mathrm{L}_{\circ} \\
\mathrm{cm}\end{array}$ & $\begin{array}{l}\mathrm{H}_{\circ} \\
\mathrm{cm}\end{array}$ & $\mathrm{H}_{\mathrm{o}} / \mathrm{L}_{\mathrm{o}}$ & $\begin{array}{l}\mathrm{H}_{\mathrm{b}} \\
\mathrm{cm}\end{array}$ & $\begin{array}{l}\mathrm{h}_{\mathrm{b}} \\
\mathrm{cm}\end{array}$ \\
\hline 1 & 0.10 & 1.2 & 224.6 & 10.0 & 0.045 & 10.0 & 8.3 \\
\hline
\end{tabular}

Table All

Results of Maruyama et a1. (1983)

\begin{tabular}{|c|c|c|c|c|c|c|c|}
\hline Run & m & $\begin{array}{c}\mathrm{T} \\
\mathrm{sec}\end{array}$ & $\begin{array}{c}\mathrm{L}_{\circ} \\
\mathrm{m}\end{array}$ & $\begin{array}{c}\mathrm{H}_{\mathrm{o}} \\
\mathrm{m}\end{array}$ & $\mathrm{H}_{0} / \mathrm{L}_{0}$ & $\begin{array}{r}\mathrm{H}_{\mathrm{b}} \\
\text { mII }\end{array}$ & $\begin{array}{c}\mathrm{h}_{\mathrm{b}} \\
\mathrm{m}\end{array}$ \\
\hline 1 & 0.034 & 3.1 & 14.99 & 1.37 & 0.091 & 1.29 & 2.0 \\
\hline
\end{tabular}

Table A12

Results of Visser (1982)

\begin{tabular}{|c|c|c|c|c|c|c|c|c|}
\hline Run & $\mathrm{m}$ & $\begin{array}{c}\mathrm{T} \\
\mathrm{sec}\end{array}$ & $\begin{array}{l}\mathrm{L}_{\circ} \\
\mathrm{cm}\end{array}$ & $\begin{array}{l}\mathrm{H}_{\mathrm{o}} \\
\mathrm{cm}\end{array}$ & $\mathrm{H}_{0} / \mathrm{L}_{0}$ & $\begin{array}{l}\mathrm{H}_{\mathrm{b}} \\
\mathrm{cm}\end{array}$ & $\begin{array}{l}\mathrm{h}_{\mathrm{b}} \\
\mathrm{cm}\end{array}$ & $\begin{array}{l}\mathrm{X}_{\mathrm{p}} \\
\mathrm{cm}\end{array}$ \\
\hline 1 & 0.10 & 2.01 & 630.1 & 9.8 & 0.016 & 10.5 & 10.4 & 59.5 \\
\hline 2 & 0.10 & 1.00 & 156.0 & 10.2 & 0.065 & 10.0 & 10.9 & 58.5 \\
\hline 3 & 0.10 & 1.00 & 156.0 & 9.6 & 0.062 & 9.7 & 11.4 & 56.4 \\
\hline 4 & 0.05 & 1.02 & 162.3 & 8.5 & 0.052 & 9.1 & 11.0 & 60.2 \\
\hline 5 & 0.05 & 1.85 & 533.8 & 7.5 & 0.014 & 10.8 & 11.6 & 69.8 \\
\hline 6 & 0.05 & 0.70 & 76.4 & 6.0 & 0.079 & 5.8 & 8.8 & 34.9 \\
\hline 7 & 0.05 & 1.02 & 162.3 & 8.5 & 0.052 & 9.0 & 12.2 & 60.5 \\
\hline
\end{tabular}

Table A13

Results of Stive (1985)

\begin{tabular}{|c|c|c|c|c|c|c|c|}
\hline Run & $\mathrm{m}$ & $\begin{array}{c}\mathrm{T} \\
\mathrm{sec}\end{array}$ & $\begin{array}{c}\mathrm{L}_{\circ} \\
\mathrm{m}\end{array}$ & $\begin{array}{c}\mathrm{H}_{\circ} \\
\mathrm{m}\end{array}$ & $\mathrm{H}_{\mathrm{o}} / \mathrm{L}_{\mathrm{o}}$ & $\begin{array}{c}\mathrm{H}_{\mathrm{b}} \\
\mathrm{m}\end{array}$ & $\begin{array}{c}\mathrm{h}_{\mathrm{b}} \\
\mathrm{m}\end{array}$ \\
\hline 1 & 0.025 & 1.8 & 5.05 & 0.16 & 0.032 & 0.18 & 0.2 \\
\hline 2 & 0.025 & 5.0 & 39.00 & 1.21 & 0.031 & 1.50 & 1.9 \\
\hline
\end{tabular}




\section{APPENDIX B: TRACINGS OF WAVE FORMS}

The waveforms in this appendix were traced from a video monitor during analysis of breaker data. The waveforms shown represent a typical wave at incipient breaking for selected classes of cases. 

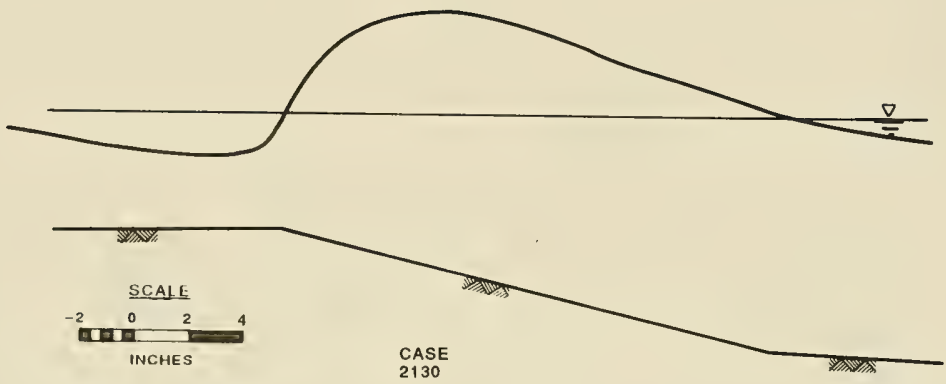

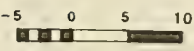

CENTIMETERS

Figure B1. Case 2130 at incipient breaking

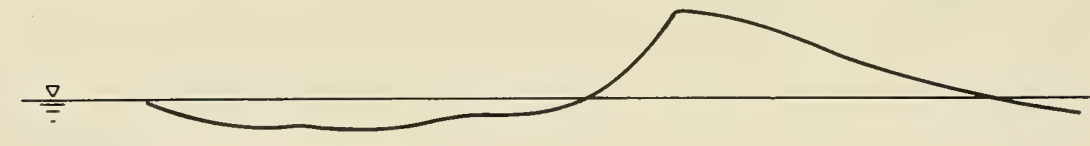

Wus

\$nes

जenter

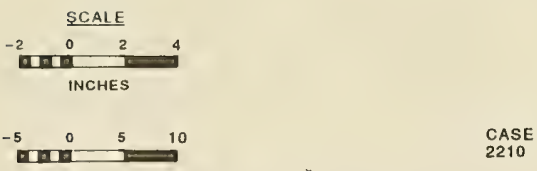

CENTIMETERS

Figure B2. Case 2210 at incipient breaking 


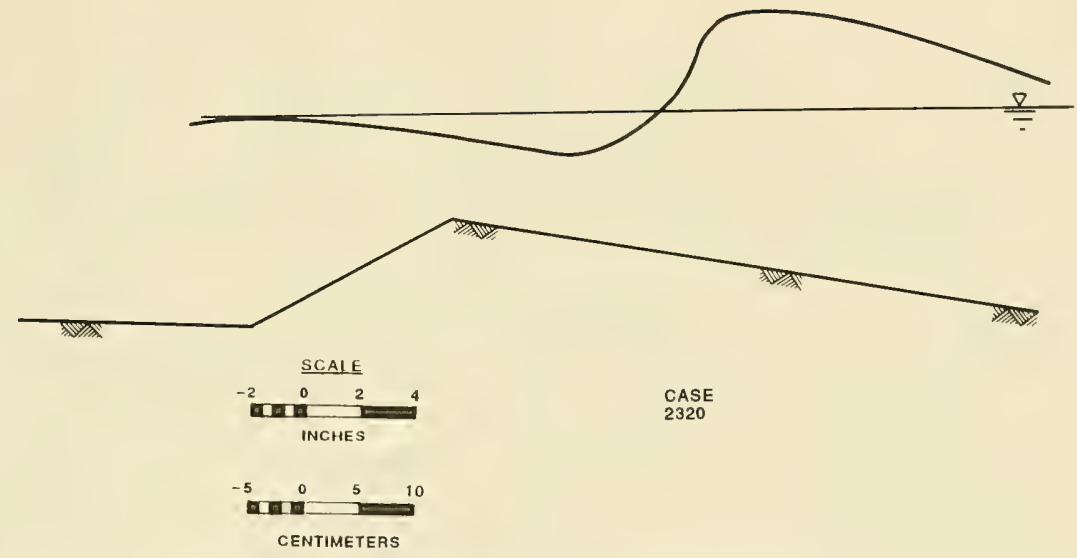

Figure B3. Case 2320 at incipient breaking
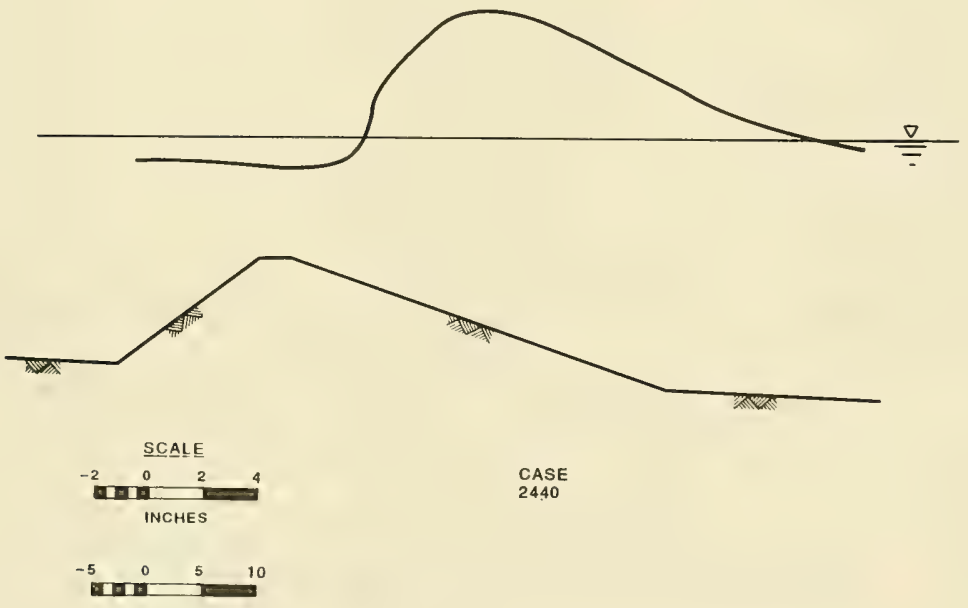

CENTIMETERS

Figure B4. Case 2440 at incipient breaking 

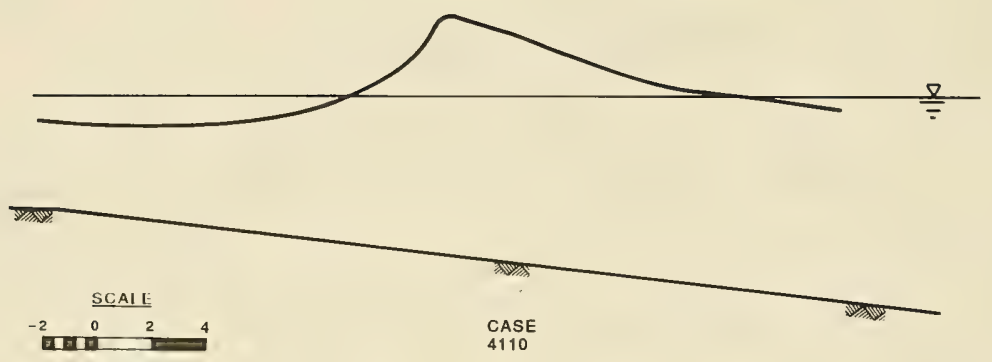

INCHES

4110

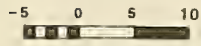

CENTIMETERS

Figure B5. Case 4110 at incipient breaking
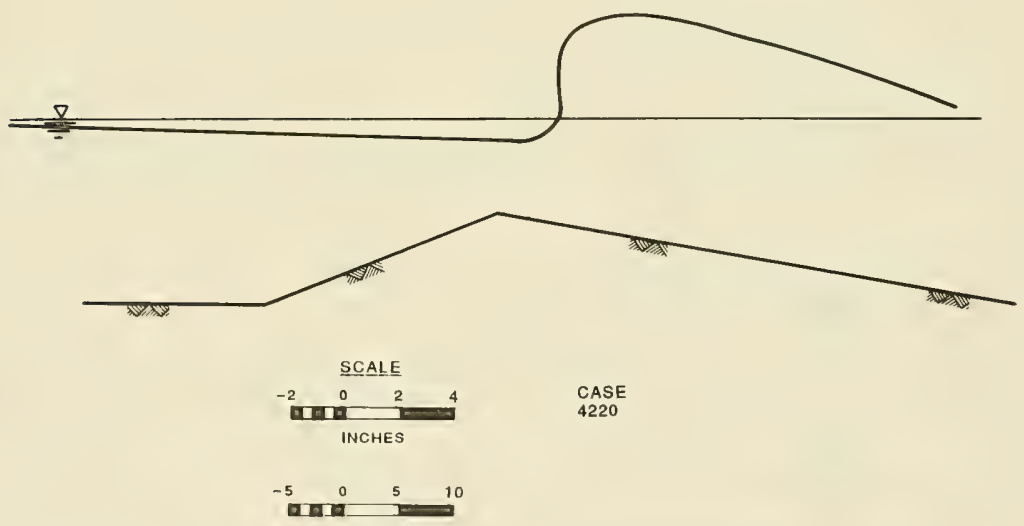

CENTIMETERS

Figure B6. Case 4220 at incipient breaking 


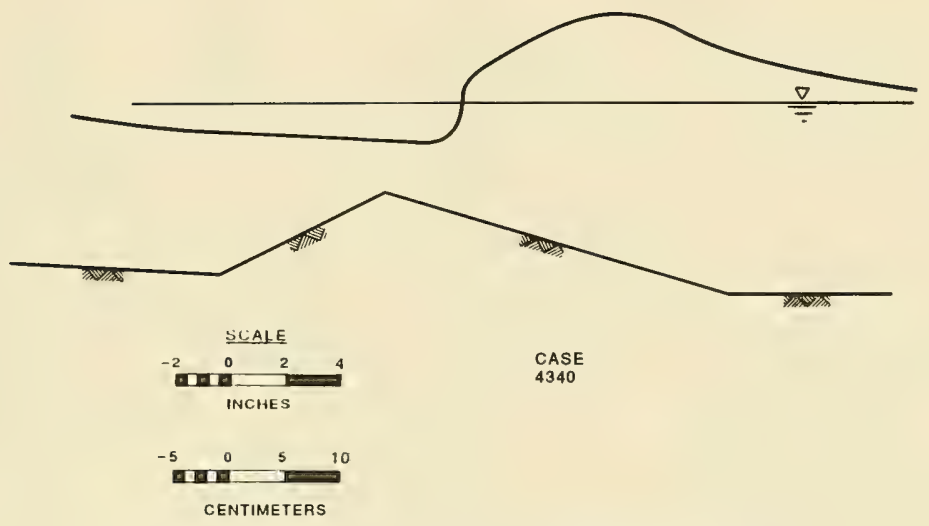

Figure B7. Case 4340 at incipient breaking
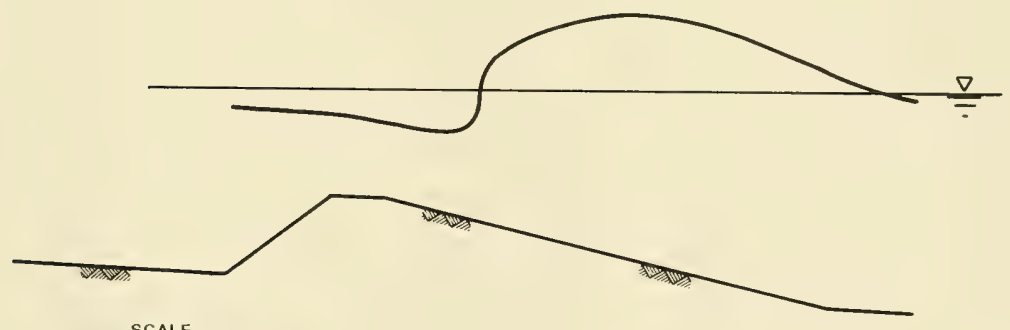

SCALE

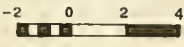

INCHES

$\begin{array}{rrr}-5 & 0 & 5\end{array}$

CASE

CENTIMETERS

Figure B8. Case 4430 at incipient breaking 


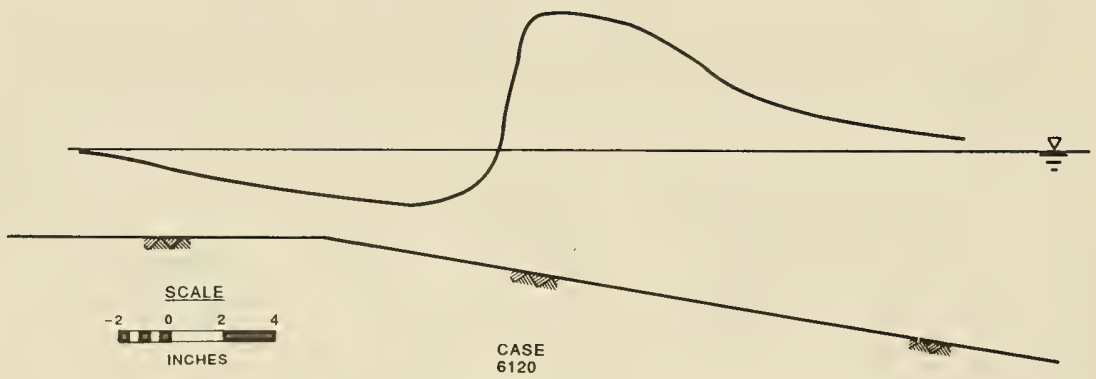

$\begin{array}{lrr}-5 \quad 0 & 5 & 10 \\ \text { मात्व } & =\end{array}$

CENTIMETERS

Figure B9. Case 6120 at incipient breaking

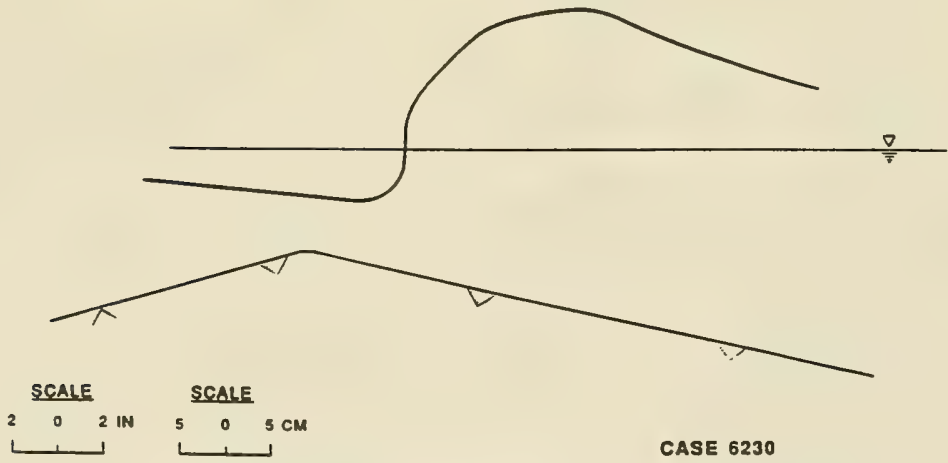

Figure B10. Case 6230 at incipient breaking 

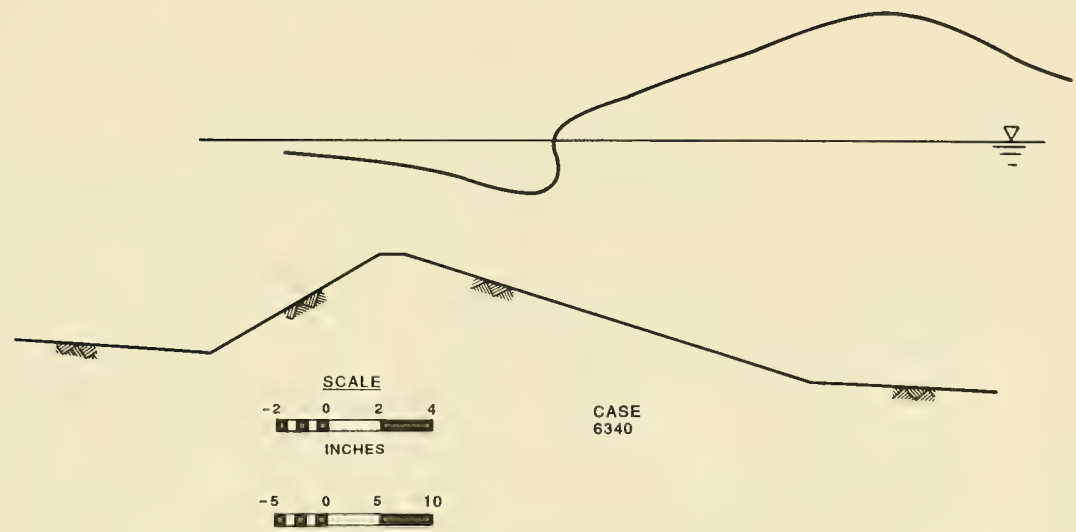

CENTIMETERS

Figure B11. Case 6340 at incipient breaking
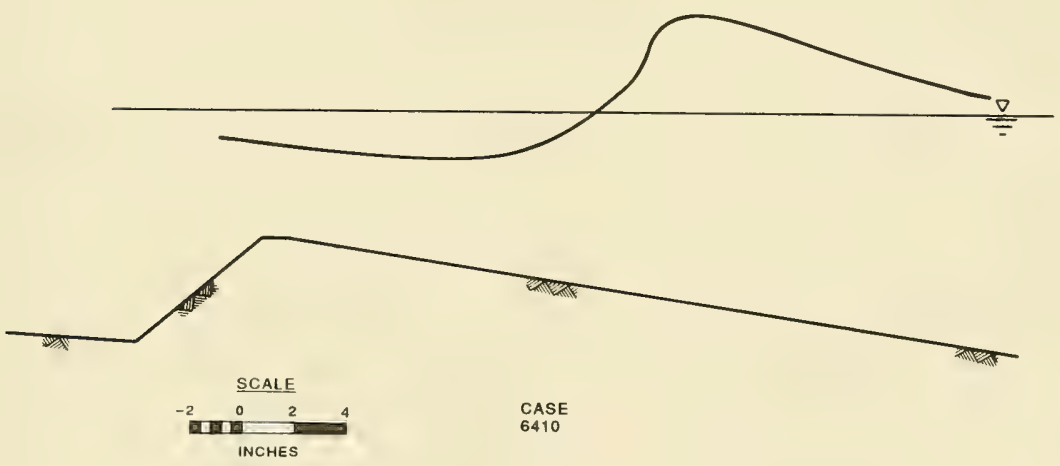

खणत 5

CENTIMETERS

Figure B12. Case 6410 at incipient breaking 


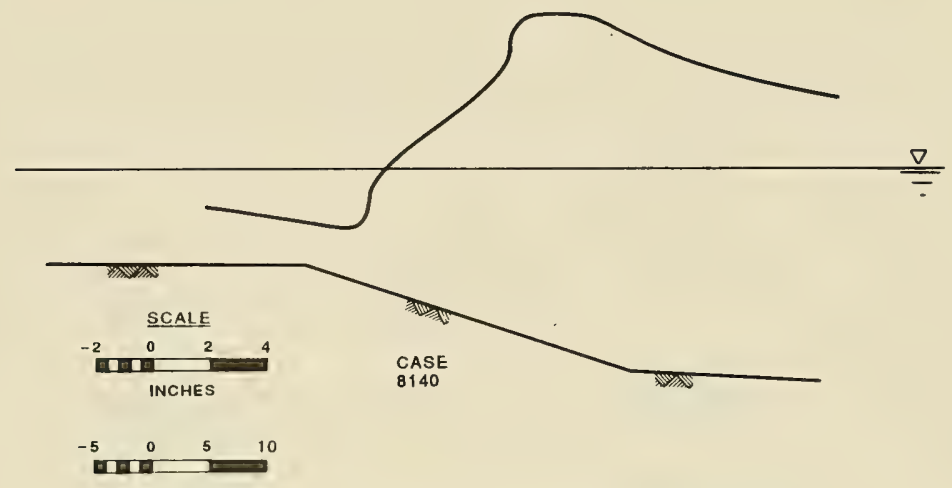

CENTIMETERS

Figure B13. Case 8140 at incipient breaking
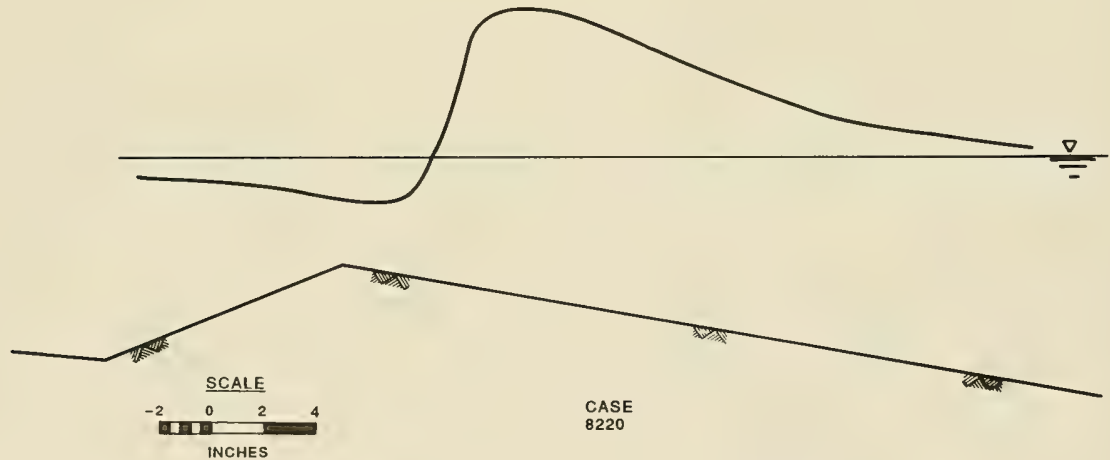

INCHES

$\begin{array}{rrr}-5 \quad 0 & 5 & 10 \\ 0 & 0\end{array}$

CENTIMETERS

Figure B14. Case 8220 at incipient breaking 

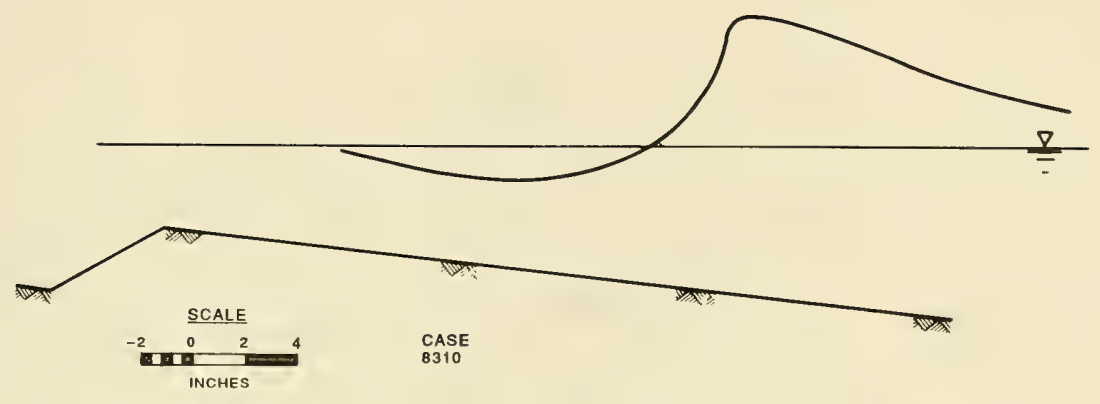

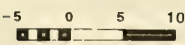

CENTIMETERS

Figure B15. Case 8310 at incipient breaking
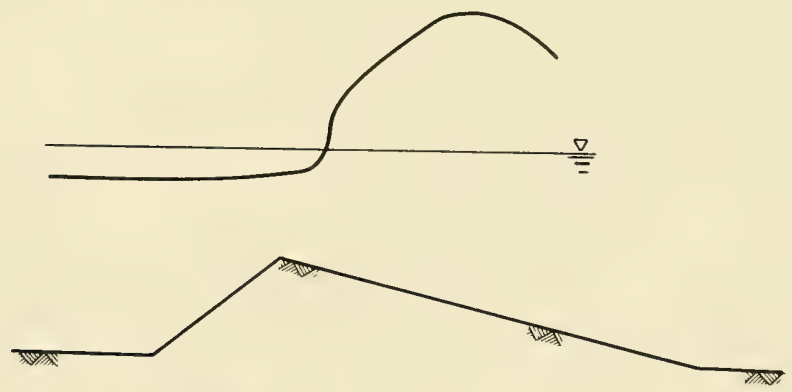

SCAI E:

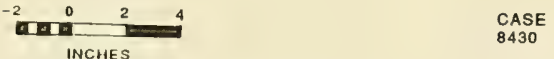

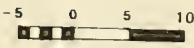

CENTIMETERS

Figure B16. Case 8430 at incipient breaking 

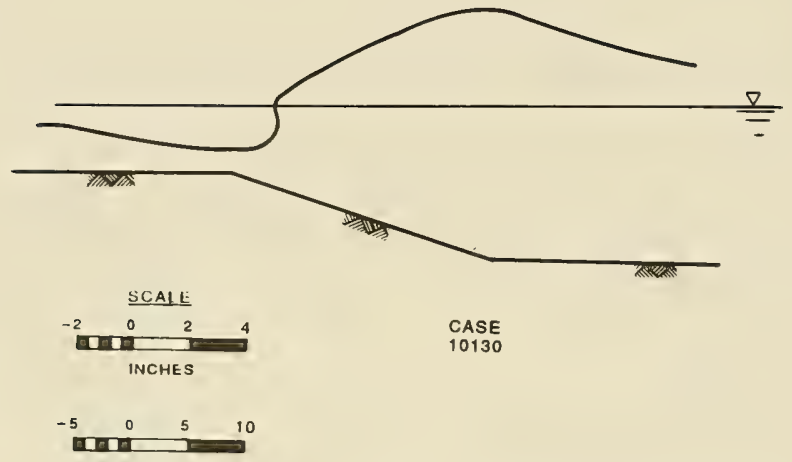

CENTIMETERS

Figure B17. Case 10130 at incipient breaking

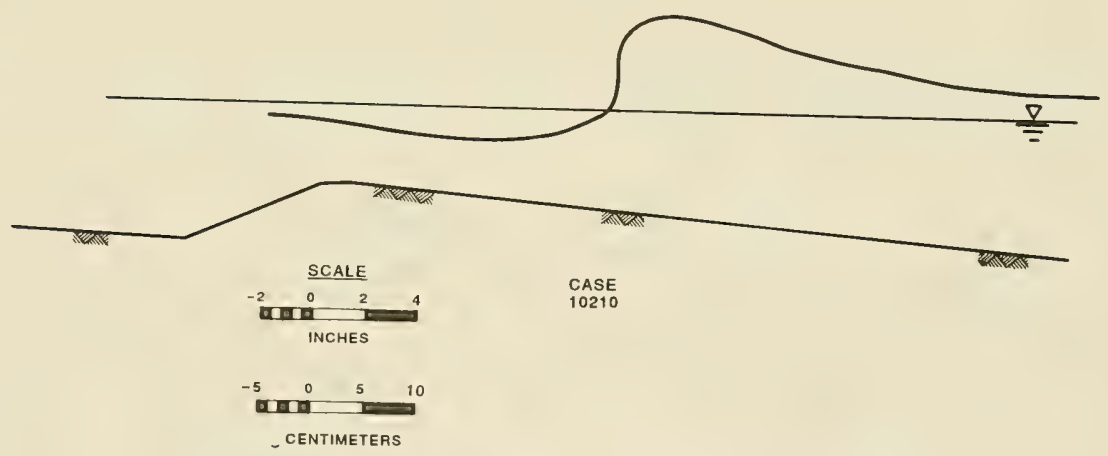

Figure B18. Case 10210 at incipient breaking 


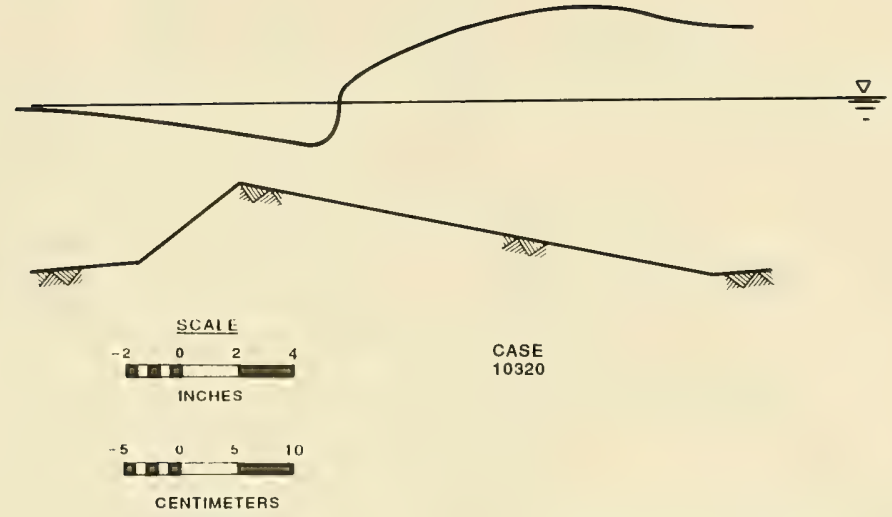

Figure B19. Case 10320 at incipient breaking

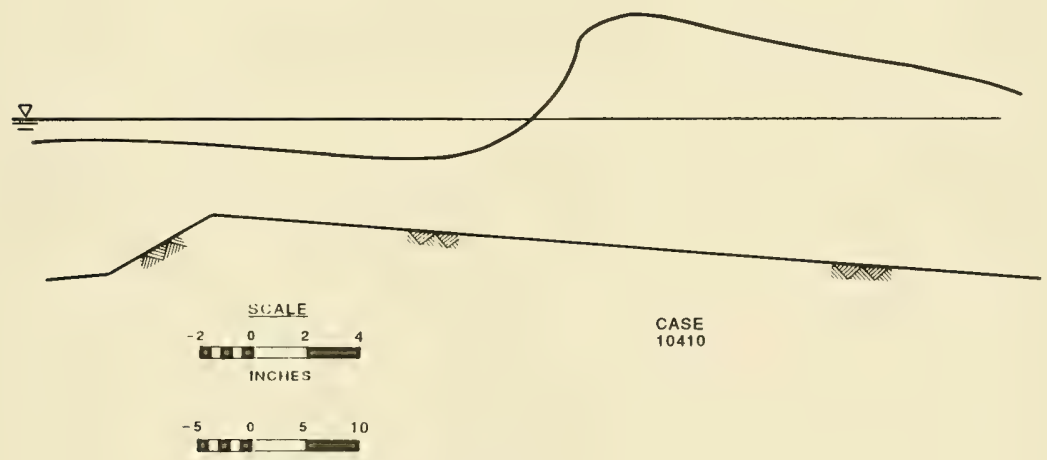

CENTIMETERS

Figure B20. Case 10410 at incipient breaking 



\section{APPENDIX C: WAVE HEIGHT DATA}

MONOCHROMATIC TESTS

KEY:

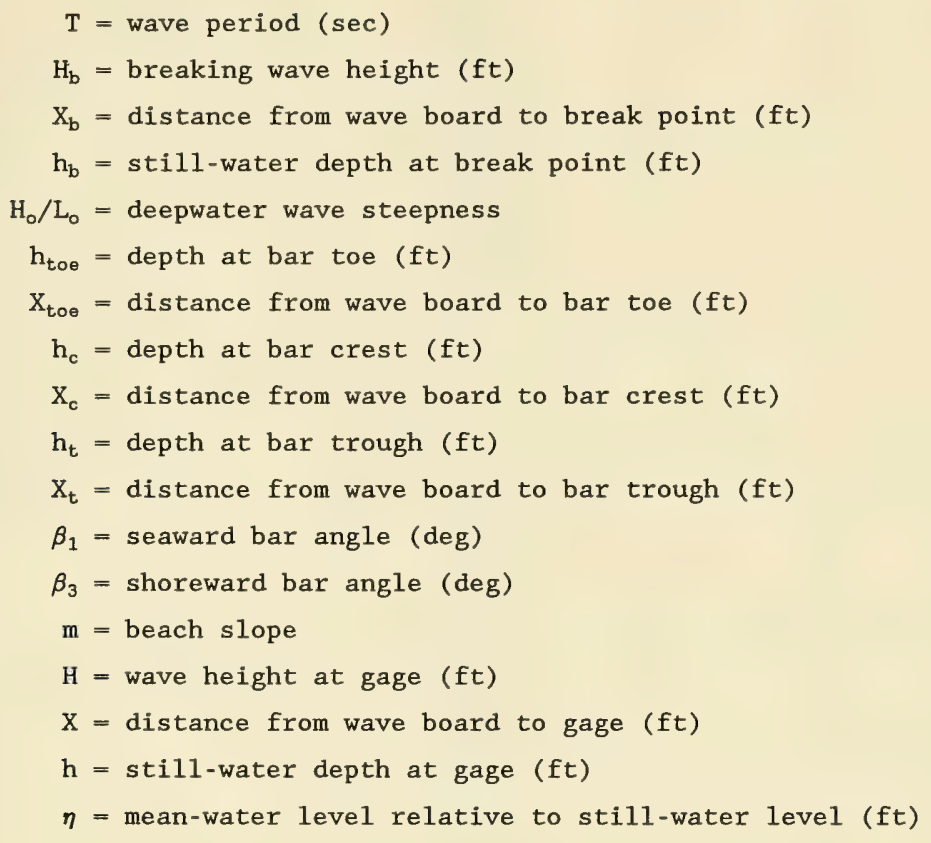

$\begin{array}{llll}0.47 & 30.00 & 1.25 & -0.016 \\ 0.45 & 79.29 & 0.93 & 0.006 \\ 0.44 & 81.00 & 0.85 & -0.003 \\ 0.47 & 83.75 & 0.61 & -0.041 \\ 0.14 & 88.04 & 0.30 & -0.025 \\ 0.12 & 90.08 & 0.30 & 0.012 \\ 0.13 & 93.00 & 0.30 & 0.043 \\ 0.12 & 97.00 & 0.29 & 0.054\end{array}$


Case 2120: $T=1.02 ; \mathrm{H}_{\mathrm{b}}=0.45 ; \mathrm{X}_{\mathrm{b}}=86.08 ; \mathrm{h}_{\mathrm{b}}=0.48 ; \mathrm{H}_{\mathrm{o}} / \mathrm{L}_{\mathrm{o}}=0.095 ;$

$h_{\text {toe }}=0.73 ; x_{\text {toe }}=85.00 ; h_{c}=0.30 ; x_{c}=87.13 ;$

$h_{\mathrm{t}}=0.30 ; \mathrm{X}_{\mathrm{t}}=96.83 ; \beta_{1}=10 ; \beta_{3}=0$.

\section{$\mathrm{H}$}

$\mathrm{X}$

h

$\eta$

0.47

30.00

1.25

$-0.010$

0.46

82.75

0.81

$-0.004$

0.43

84.50

0.75

$-0.018$

0.43

86.25

0.44

$-0.054$

0.15

89.00

0.30

0.029

0.13

91.50

0.30

0.019

0.14

94.00

0.30

0.043

0.12

99.00

0.28

0.060

Case 2130: $T=1.02 ; H_{b}=0.37 ; X_{b}=86.64 ; h_{b}=0.43 ; H_{o} / L_{o}=0.094 ;$

$h_{\text {toe }}=0.69 ; X_{\text {toe }}=85.79 ; h_{c}=0.31 ; x_{c}=87.13$;

$\mathrm{h}_{\mathrm{t}}=0.30 ; \mathrm{X}_{\mathrm{t}}=96.83 ; \beta_{1}=15 ; \beta_{3}=0$.

$\mathrm{H}$

$\mathrm{X}$

$\mathrm{h}$

$\eta$

0.47

30.00

1.25

$-0.024$

0.47

83.29

0.78

$-0.005$

0.44

85.00

0.73

$-0.025$

0.46

85.96

0.57

$-0.049$

0.15

89.50

0.29

0.031

0.12

91.50

0.29

0.014

0.14

94.00

0.29

0.040

0.11

99.00

0.28

0.050

Case 2140: $T=1.01 ; \mathrm{H}_{\mathrm{b}}=0.39 ; \mathrm{X}_{\mathrm{b}}=86.73 ; \mathrm{h}_{\mathrm{b}}=0.45 ; \mathrm{H}_{\mathrm{o}} / \mathrm{L}_{\mathrm{o}}=0.091 ;$

$\mathrm{h}_{\text {toe }}=0.63 ; \mathrm{x}_{\text {toe }}=86.17 ; \mathrm{h}_{\mathrm{c}}=0.31 ; \mathrm{x}_{\mathrm{c}}=87.13$;

$\mathrm{h}_{\mathrm{t}}=0.27 ; \mathrm{x}_{\mathrm{t}}=96.83 ; \beta_{1}=20 ; \beta_{3}=0$.

$\mathrm{H}$

$\mathrm{X}$

0.44

30.00

1. 25

$-0.024$

0.43

83.75

0.72

$-0.022$

0.42

85.50

0.70

$-0.027$

0.42

86.50

0.50

$-0.031$

0.10

89.50

0.29

0.008

0.13

91.50

0.29

0.029

0.14

94.00

0.29

0.041

0.11

99.00

0.28

0.044 
Case 2150: $\quad T=1.00 ; H_{b}=0.00 ; X_{b}=0.00 ; h_{b}=0.00 ; H_{o} / L_{o}=0.090 ;$

$\mathrm{h}_{\text {toe }}=0.66 ; \mathrm{x}_{\text {toe }}=86.50 ; \mathrm{h}_{\mathrm{c}}=0.31 ; \mathrm{x}_{\mathrm{c}}=87.13 ;$

$h_{\mathrm{t}}=0.28 ; \mathrm{x}_{\mathrm{t}}=96.83 ; \beta_{1}=30 ; \beta_{3}=0$.

$\mathrm{H} \quad \mathrm{X} \quad \mathrm{h} \quad \eta$

$\begin{array}{llll}0.47 & 30.00 & 1.25 & -0.003 \\ 0.41 & 83.75 & 0.76 & -0.025 \\ 0.42 & 85.50 & 0.70 & -0.025 \\ 0.42 & 86.58 & 0.53 & -0.030 \\ 0.12 & 89.50 & 0.29 & 0.010 \\ 0.14 & 91.50 & 0.29 & 0.031 \\ 0.13 & 94.00 & 0.28 & 0.041 \\ 0.11 & 99.00 & 0.28 & 0.048\end{array}$

Case 2160: $T=1.00 ; H_{b}=0.00 ; X_{b}=0.00 ; h_{b}=0.00 ; H_{o} / L_{o}=0.090 ;$

$\mathrm{h}_{\text {toe }}=0.66 ; \mathrm{x}_{\text {toe }}=86.67 ; \mathrm{h}_{\mathrm{c}}=0.31 ; \mathrm{x}_{\mathrm{c}}=87.13$;

$h_{\mathrm{t}}=0.28 ; \mathrm{x}_{\mathrm{t}}=96.83 ; \beta_{1}=40 ; \beta_{3}=0$.

$\mathrm{H} \quad \mathrm{X} \quad \mathrm{h} \quad \eta$

$\begin{array}{llll}0.48 & 30.00 & 1.25 & 0.002 \\ 0.42 & 83.75 & 0.77 & -0.012 \\ 0.43 & 85.46 & 0.71 & -0.015 \\ 0.44 & 86.75 & 0.50 & -0.020 \\ 0.11 & 89.50 & 0.30 & 0.014 \\ 0.12 & 91.50 & 0.30 & 0.040 \\ 0.15 & 94.00 & 0.29 & 0.051 \\ 0.10 & 99.00 & 0.28 & 0.055\end{array}$

Case 2211: $T=1.00 ; H_{b}=0.00 ; X_{b}=0.00 ; h_{b}=0.00 ; H_{o} / L_{o}=0.090 ;$

$\mathrm{h}_{\text {toe }}=0.78 ; \mathrm{X}_{\text {toe }}=83.08 ; \mathrm{h}_{\mathrm{c}}=0.29 ; \mathrm{X}_{\mathrm{c}}=88.25$;

$h_{\mathrm{t}}=0.60 ; \mathrm{X}_{\mathrm{t}}=89.08 ; \beta_{1}=5 ; \beta_{3}=20$.

$\begin{array}{cccc}\mathrm{H} & \mathrm{X} & \mathrm{h} & \eta \\ 0.44 & 30.00 & 1.25 & -0.012 \\ 0.42 & 81.00 & 0.96 & -0.007 \\ 0.42 & 82.75 & 0.88 & -0.003 \\ 0.43 & 85.35 & 0.79 & -0.015 \\ 0.10 & 88.75 & 0.65 & 0.013 \\ 0.20 & 90.75 & 0.59 & 0.032 \\ 0.16 & 93.75 & 0.48 & 0.029 \\ 0.17 & 97.25 & 0.33 & 0.032\end{array}$


Case 2212: $T=1.02 ; H_{b}=0.39 ; X_{b}=82.35 ; h_{b}=0.60 ; H_{o} / L_{o}=0.089 ;$

$h_{\text {toe }}=0.87 ; \mathrm{X}_{\text {toe }}=80.63 ; \mathrm{h}_{\mathrm{c}}=0.30 ; \mathrm{X}_{\mathrm{c}}=85.83 ;$

$h_{\mathrm{t}}=0.67 ; \mathrm{X}_{\mathrm{t}}=86.58 ; \beta_{1}=5 ; \beta_{3}=20$.

$\begin{array}{cccc}\mathrm{H} & \mathrm{X} & \mathrm{h} & \eta \\ 0.44 & 30.00 & 1.25 & -0.012 \\ 0.41 & 78.25 & 0.96 & 0.007 \\ 0.41 & 79.92 & 0.88 & 0.018 \\ 0.45 & 83.38 & 0.79 & -0.007 \\ 0.14 & 87.50 & 0.65 & 0.028 \\ 0.20 & 89.50 & 0.59 & 0.025 \\ 0.17 & 92.50 & 0.48 & 0.029 \\ 0.17 & 97.50 & 0.33 & 0.032\end{array}$

Case 2220: $T=1.02 ; H_{b}=0.41 ; x_{b}=85.91 ; h_{b}=0.42 ; H_{o} / L_{o}=0.091 ;$

$h_{\text {toe }}=0.73 ; x_{\text {toe }}=84.88 ; h_{c}=0.26 ; x_{c}=86.83$;

$h_{\mathrm{t}}=0.64 ; \mathrm{x}_{\mathrm{t}}=87.58 ; \beta_{1}=10 ; \beta_{3}=20$.

$\begin{array}{lllll}\mathrm{H} & \mathrm{X} & \mathrm{h} & \eta\end{array}$

$\begin{array}{llll}0.45 & 30.00 & 1.25 & -0.003\end{array}$

$\begin{array}{lllll}0.46 & 82.75 & 0.81 & -0.003\end{array}$

$\begin{array}{llll}0.45 & 84.50 & 0.74 & -0.007\end{array}$

$\begin{array}{llll}0.41 & 86.08 & 0.40 & -0.019\end{array}$

$\begin{array}{lllll}0.18 & 88.29 & 0.62 & 0.011\end{array}$

$\begin{array}{llll}0.16 & 90.25 & 0.57 & 0.017\end{array}$

$\begin{array}{llll}0.17 & 93.25 & 0.46 & 0.017\end{array}$

$\begin{array}{llll}0.18 & 97.30 & 0.33 & 0.020\end{array}$

Case 2230: $T=1.02 ; H_{b}=0.39 ; X_{b}=87.27 ; h_{b}=0.43 ; H_{o} / L_{o}=0.094 ;$

$\mathrm{h}_{\text {toe }}=0.67 ; \mathrm{x}_{\text {toe }}=86.50 ; \mathrm{h}_{\mathrm{c}}=0.30 ; \mathrm{x}_{\mathrm{c}}=87.75 ;$

$h_{\mathrm{t}}=0.62 ; \mathrm{x}_{\mathrm{t}}=88.46 ; \beta_{1}=15 ; \beta_{3}=20$.

$\mathrm{H}$

$\mathrm{X}$

$\mathrm{h}$

$\eta$

0.45

30.00

1.25

0.000

0.45

83.75

0.77

0.001

0.46

85.50

0.71

0.001

0.44

86.92

0.47

$-0.016$

0.23

89.25

0.59

0.016

0.21

91.25

0.52

0.017

0.19

94.25

0.44

0.026

0.18

98.25

0.30

0.028 
Case 2240: $T=1.03 ; \mathrm{H}_{\mathrm{b}}=0.38 ; \mathrm{X}_{\mathrm{b}}=87.32 ; \mathrm{h}_{\mathrm{b}}=0.46 ; \mathrm{H}_{\mathrm{o}} / \mathrm{L}_{\mathrm{o}}=0.091 ;$

$\mathrm{h}_{\text {toe }}=0.67 ; \mathrm{x}_{\text {toe }}=86.83 ; \mathrm{h}_{\mathrm{c}}=0.30 ; \mathrm{x}_{\mathrm{c}}=87.75 ;$

$h_{\mathrm{t}}=0.62 ; \mathrm{X}_{\mathrm{t}}=88.46 ; \beta_{1}=20 ; \beta_{3}=20$.

$\begin{array}{cccc}\mathrm{H} & \mathrm{X} & \mathrm{h} & \boldsymbol{\eta} \\ 0.46 & 30.00 & 1.25 & 0.002 \\ 0.46 & 83.75 & 0.77 & 0.010 \\ 0.49 & 85.50 & 0.71 & -0.009 \\ 0.46 & 87.00 & 0.52 & 0.000 \\ 0.24 & 89.25 & 0.59 & 0.016 \\ 0.21 & 91.25 & 0.52 & 0.019 \\ 0.20 & 94.25 & 0.44 & 0.026 \\ 0.20 & 98.25 & 0.30 & 0.026\end{array}$

Case 2250: $T=1.00 ; \mathrm{H}_{\mathrm{b}}=0.00 ; \mathrm{X}_{\mathrm{b}}=0.00 ; \mathrm{h}_{\mathrm{b}}=0.00 ; \mathrm{H}_{\mathrm{o}} / \mathrm{L}_{\mathrm{o}}=0.090 ;$

$\mathrm{h}_{\text {toe }}=0.66 ; \mathrm{x}_{\mathrm{toe}}=87.21 ; \mathrm{h}_{\mathrm{c}}=0.30 ; \mathrm{x}_{\mathrm{c}}=87.75$;

$h_{\mathrm{t}}=0.63 ; \mathrm{x}_{\mathrm{t}}=88.46 ; \beta_{1}=30 ; \beta_{3}=20$.

$\begin{array}{cccc}\mathrm{H} & \mathrm{X} & \mathrm{h} & \eta \\ 0.43 & 30.00 & 1.25 & -0.005 \\ 0.40 & 84.25 & 0.75 & -0.016 \\ 0.44 & 86.00 & 0.68 & -0.015 \\ 0.47 & 87.40 & 0.50 & -0.021 \\ 0.26 & 89.13 & 0.60 & -0.001 \\ 0.21 & 91.25 & 0.52 & 0.006 \\ 0.18 & 94.25 & 0.44 & 0.016 \\ 0.18 & 98.25 & 0.29 & 0.024\end{array}$

Case 2260: $T=1.00 ; H_{b}=0.00 ; X_{b}=0.00 ; h_{b}=0.00 ; H_{o} / L_{o}=0.090 ;$

$h_{\text {toe }}=0.67 ; X_{\text {toe }}=86.92 ; h_{c}=0.29 ; X_{c}=87.33 ;$

$h_{\mathrm{t}}=0.63 ; \mathrm{X}_{\mathrm{t}}=88.08 ; \beta_{1}=40 ; \beta_{3}=20$.

$\mathrm{H}$ $\mathrm{X}$

h

$\eta$

$\begin{array}{llll}0.44 & 30.00 & 1.25 & -0.010 \\ 0.38 & 84.25 & 0.76 & -0.022 \\ 0.35 & 86.00 & 0.68 & -0.027 \\ 0.52 & 87.04 & 0.41 & -0.030 \\ 0.25 & 88.63 & 0.61 & 0.004 \\ 0.20 & 90.67 & 0.54 & 0.007 \\ 0.19 & 93.67 & 0.45 & 0.019 \\ 0.20 & 97.67 & 0.32 & 0.025\end{array}$


Case 2310: $T=1.02 ; H_{b}=0.39 ; X_{b}=84.13 ; h_{b}=0.58 ; H_{o} / L_{o}=0.091 ;$

$\mathrm{h}_{\mathrm{tO \theta}}=0.83 ; \mathrm{X}_{\mathrm{tO \theta}}=82.17 ; \mathrm{h}_{\mathrm{c}}=0.31 ; \mathrm{X}_{\mathrm{c}}=87.25 ;$

$h_{\mathrm{t}}=0.64 ; \mathrm{x}_{\mathrm{t}}=87.75 ; \beta_{1}=5 ; \beta_{3}=30$.

$\begin{array}{cccc}\text { H } & \mathrm{X} & \mathrm{h} & \eta \\ 0.45 & 30.00 & 1.25 & -0.003 \\ 0.42 & 79.75 & 0.90 & 0.013 \\ 0.41 & 81.50 & 0.83 & 0.015 \\ 0.44 & 84.46 & 0.55 & -0.007 \\ 0.14 & 88.67 & 0.61 & 0.026 \\ 0.20 & 90.67 & 0.55 & 0.025 \\ 0.17 & 93.67 & 0.45 & 0.029 \\ 0.20 & 97.67 & 0.32 & 0.034\end{array}$

Case 2320: $T=1.02 ; \mathrm{H}_{\mathrm{b}}=0.43 ; \mathrm{X}_{\mathrm{b}}=86.17 ; \mathrm{h}_{\mathrm{b}}=0.47 ; \mathrm{H}_{\mathrm{o}} / \mathrm{L}_{\mathrm{o}}=0.088 ;$

$h_{\text {toe }}=0.72 ; X_{\text {toe }}=85.25 ; h_{c}=0.29 ; x_{c}=87.17 ;$

$h_{\mathrm{t}}=0.64 ; \mathrm{X}_{\mathrm{t}}=87.67 ; \beta_{1}=10 ; \beta_{3}=30$.

$\mathrm{H}$

$\mathrm{X}$

h

$\eta$

$\begin{array}{lllr}0.43 & 30.00 & 1.25 & -0.013 \\ 0.47 & 83.25 & 0.80 & 0.002 \\ 0.47 & 85.00 & 0.73 & 0.002 \\ 0.40 & 86.38 & 0.43 & -0.033 \\ 0.23 & 88.67 & 0.61 & 0.014 \\ 0.19 & 90.67 & 0.55 & 0.009 \\ 0.19 & 93.67 & 0.45 & 0.017 \\ 0.21 & 97.67 & 0.32 & 0.019\end{array}$

Case 2330: $T=1.02 ; H_{b}=0.38 ; X_{b}=86.91 ; h_{b}=0.42 ; H_{o} / L_{o}=0.093 ;$

$\mathrm{h}_{\text {toe }}=0.68 ; \mathrm{x}_{\text {toe }}=86.17 ; \mathrm{h}_{\mathrm{c}}=0.29 ; \mathrm{x}_{\mathrm{c}}=87.38 ;$

$\mathrm{h}_{\mathrm{t}}=0.64 ; \mathrm{x}_{\mathrm{t}}=87.83 ; \beta_{1}=15 ; \beta_{3}=30$.

$\begin{array}{cccc}\mathrm{H} & \mathrm{X} & \mathrm{h} & \eta \\ 0.46 & 30.00 & 1.25 & 0.018 \\ 0.44 & 83.75 & 0.77 & 0.007 \\ 0.47 & 85.50 & 0.71 & 0.006 \\ 0.47 & 87.00 & 0.37 & -0.017 \\ 0.25 & 88.67 & 0.61 & 0.015 \\ 0.19 & 90.67 & 0.54 & 0.017 \\ 0.19 & 93.67 & 0.45 & 0.029 \\ 0.19 & 97.67 & 0.32 & 0.029\end{array}$


Case 2340: $T=1.02 ; H_{b}=0.38 ; X_{b}=86.73 ; h_{b}=0.47 ; H_{o} / L_{o}=0.093 ;$

$h_{\text {toe }}=0.67 ; X_{\text {toe }}=86.38 ; h_{c}=0.29 ; x_{c}=87.21 ;$

$h_{\mathrm{t}}=0.64 ; \mathrm{X}_{\mathrm{t}}=87.75 ; \beta_{1}=20 ; \beta_{3}=30$.

$\begin{array}{cccc}\text { H } & \text { X } & \text { h } & \eta \\ 0.46 & 30.00 & 1.25 & 0.013 \\ 0.42 & 84.25 & 0.76 & 0.002 \\ 0.39 & 86.00 & 0.68 & -0.010 \\ 0.50 & 87.00 & 0.38 & -0.016 \\ 0.26 & 88.67 & 0.61 & 0.014 \\ 0.22 & 90.67 & 0.54 & 0.016 \\ 0.21 & 93.67 & 0.45 & 0.028 \\ 0.21 & 97.67 & 0.32 & 0.028\end{array}$

Case 2350: $T=1.00 ; H_{b}=0.00 ; X_{b}=0.00 ; h_{b}=0.00 ; H_{o} / L_{o}=0.090 ;$

$\mathrm{h}_{\text {toe }}=0.67 ; \mathrm{x}_{\text {toe }}=86.67 ; \mathrm{h}_{\mathrm{c}}=0.29 ; \mathrm{x}_{\mathrm{c}}=87.25$;

$\mathrm{h}_{\mathrm{t}}=0.64 ; \mathrm{x}_{\mathrm{t}}=87.75 ; \beta_{1}=30 ; \beta_{3}=30$.

$\begin{array}{cccc}\mathrm{H} & \mathrm{X} & \mathrm{h} & \eta \\ 0.45 & 30.00 & 1.25 & 0.001 \\ 0.41 & 84.25 & 0.76 & -0.009 \\ 0.36 & 86.00 & 0.68 & -0.020 \\ 0.52 & 87.00 & 0.40 & -0.025 \\ 0.24 & 88.67 & 0.61 & 0.013 \\ 0.22 & 90.67 & 0.54 & 0.014 \\ 0.19 & 93.67 & 0.45 & 0.026 \\ 0.20 & 96.83 & 0.34 & 0.029\end{array}$

Case 2360: $T=1.00 ; H_{b}=0.00 ; X_{b}=0.00 ; h_{b}=0.00 ; H_{o} / L_{o}=0.090 ;$

$h_{\text {toe }}=0.68 ; x_{\text {toe }}=87.00 ; h_{c}=0.29 ; x_{c}=87.25 ;$

$h_{t}=0.64 ; X_{t}=87.75 ; \beta_{1}=40 ; \beta_{3}=30$.

$\mathrm{H}$ $\mathrm{X}$

h

$\eta$

0.44

30.00

1.25

$-0.018$

0.41

84.25

0.76

$-0.022$

0.35

86.00

0.68

$-0.033$

0.54

87.00

0.44

$-0.028$

0.23

88.63

0.61

0.001

0.21

90.67

0.54

0.007

0.19

93.67

0.45

0.016

0.20

97.67

0.32

0.024 


$\begin{array}{cccc}\text { Case } 2410: T=1.02 ; \mathrm{H}_{\mathrm{b}}=0.39 ; \mathrm{X}_{\mathrm{b}}=84 \\ \mathrm{~h}_{\text {toe }}=0.81 ; \mathrm{X}_{\text {toe }}=82.33 ; \mathrm{h}_{\mathrm{c}}=0.30 ; \mathrm{X}_{\mathrm{c}} \\ \mathrm{h}_{\mathrm{t}}=0.63 ; \mathrm{X}_{\mathrm{t}}=87.67 ; \beta_{1}=5 ; \beta_{3}=40 . \\ \mathrm{H} & \mathrm{X} & \mathrm{h} & \eta \\ 0.45 & 30.00 & 1.25 & -0.001 \\ 0.41 & 80.25 & 0.88 & 0.012 \\ 0.40 & 82.00 & 0.83 & 0.011 \\ 0.45 & 84.75 & 0.55 & -0.007 \\ 0.13 & 88.67 & 0.61 & 0.029 \\ 0.20 & 90.67 & 0.55 & 0.029 \\ 0.16 & 93.67 & 0.45 & 0.030 \\ 0.19 & 97.67 & 0.32 & 0.035\end{array}$

Case 2420: $T=1.02 ; H_{b}=0.39 ; X_{b}=87.34 ; h_{b}=0.37 ; H_{o} / L_{o}=0.090 ;$ $h_{\text {toe }}=0.69 ; x_{\text {toe }}=85.79 ; h_{c}=0.30 ; x_{c}=87.75 ;$

$h_{\mathrm{t}}=0.62 ; \mathrm{X}_{\mathrm{t}}=88.08 ; \beta_{1}=10 ; \beta_{3}=40$.

$\mathrm{H}$ $\mathrm{X}$

h

$\eta$

$\begin{array}{llll}0.44 & 30.00 & 1.25 & -0.009 \\ 0.48 & 83.50 & 0.78 & -0.003 \\ 0.45 & 85.25 & 0.72 & -0.013 \\ 0.40 & 86.67 & 0.47 & -0.024 \\ 0.16 & 89.50 & 0.59 & 0.011 \\ 0.20 & 91.50 & 0.50 & 0.019 \\ 0.20 & 94.50 & 0.43 & 0.023 \\ 0.22 & 98.50 & 0.30 & 0.024\end{array}$

Case 2430: $T=1.02 ; H_{b}=0.39 ; X_{b}=87.50 ; h_{b}=0.39 ; H_{o} / L_{o}=0.088 ;$

$h_{\text {toe }}=0.66 ; x_{\text {toe }}=86.67 ; h_{c}=0.30 ; x_{c}=87.83 ;$

$\mathrm{h}_{\mathrm{t}}=0.63 ; \mathrm{X}_{\mathrm{t}}=88.17 ; \beta_{1}=15 ; \beta_{3}=40$.

$\mathrm{H}$

$\mathrm{X}$

h

$\eta$

$\begin{array}{llll}0.44 & 30.00 & 1.25 & -0.013 \\ 0.45 & 83.75 & 0.77 & -0.012 \\ 0.47 & 85.50 & 0.70 & -0.009 \\ 0.43 & 86.92 & 0.52 & -0.020 \\ 0.22 & 89.50 & 0.59 & 0.008 \\ 0.21 & 91.50 & 0.50 & 0.015 \\ 0.19 & 94.50 & 0.43 & 0.020 \\ 0.18 & 98.50 & 0.30 & 0.024\end{array}$


Case 2440: $T=1.02 ; H_{b}=0.39 ; X_{b}=87.49 ; h_{b}=0.42 ; H_{o} / L_{o}=0.091 ;$

$\mathrm{h}_{\text {toe }}=0.66 ; \mathrm{x}_{\text {toe }}=86.96 ; \mathrm{h}_{\mathrm{c}}=0.30 ; \mathrm{x}_{\mathrm{c}}=87.83$;

$h_{t}=0.62 ; X_{t}=88.17 ; \beta_{1}=20 ; \beta_{3}=40$.

$\begin{array}{cccc}\text { H } & \mathrm{X} & \mathrm{h} & \eta \\ 0.45 & 30.00 & 1.25 & 0.016 \\ 0.39 & 84.25 & 0.75 & -0.009 \\ 0.42 & 86.00 & 0.68 & -0.019 \\ 0.47 & 87.21 & 0.50 & -0.015 \\ 0.22 & 89.50 & 0.59 & 0.006 \\ 0.21 & 91.50 & 0.50 & 0.005 \\ 0.20 & 94.50 & 0.43 & 0.019 \\ 0.18 & 98.50 & 0.30 & 0.024\end{array}$

Case 2450: $T=1.00 ; H_{b}=0.00 ; X_{b}=0.00 ; h_{b}=0.00 ; H_{o} / L_{o}=0.090 ;$

$h_{\text {toe }}=0.67 ; x_{\text {toe }}=86.58 ; h_{c}=0.30 ; x_{c}=87.13 ;$

$h_{\mathrm{t}}=0.64 ; \mathrm{X}_{\mathrm{t}}=87.46 ; \beta_{1}=30 ; \beta_{3}=40$.

$\begin{array}{lccc}\mathrm{H} & \mathrm{X} & \mathrm{h} & \eta \\ 0.45 & 30.00 & 1.25 & 0.025 \\ 0.37 & 83.75 & 0.77 & -0.009 \\ 0.41 & 85.50 & 0.70 & -0.015 \\ 0.52 & 86.75 & 0.49 & -0.011 \\ 0.19 & 89.50 & 0.59 & 0.005 \\ 0.21 & 91.50 & 0.50 & 0.003 \\ 0.20 & 94.50 & 0.43 & 0.021 \\ 0.19 & 98.50 & 0.30 & 0.027\end{array}$

Case 2460: $T=1.00 ; H_{b}=0.00 ; X_{b}=0.00 ; h_{b}=0.00 ; H_{o} / L_{o}=0.090 ;$

$h_{\text {toe }}=0.67 ; x_{\text {toe }}=86.67 ; h_{c}=0.30 ; x_{c}=87.08 ;$

$h_{t}=0.66 ; X_{t}=87.42 ; \beta_{1}=40 ; \beta_{3}=40$.

$\mathrm{H}$ $\mathrm{X}$

$\mathrm{h}$

$\eta$

$\begin{array}{llll}0.46 & 30.00 & 1.25 & 0.027\end{array}$

$\begin{array}{llll}0.37 & 83.75 & 0.77 & -0.002\end{array}$

$\begin{array}{llll}0.40 & 85.50 & 0.71 & -0.014\end{array}$

$\begin{array}{llll}0.53 & 86.79 & 0.46 & -0.003\end{array}$

$\begin{array}{llll}0.24 & 88.75 & 0.61 & 0.007\end{array}$

$\begin{array}{llll}0.22 & 90.75 & 0.54 & 0.002\end{array}$

$\begin{array}{llll}0.19 & 93.75 & 0.45 & 0.021\end{array}$

$\begin{array}{llll}0.19 & 97.75 & 0.32 & 0.028\end{array}$ 
Case 4110: $T=1.02 ; H_{b}=0.30 ; X_{b}=89.35 ; h_{b}=0.40 ; H_{o} / L_{o}=0.069 ;$

$h_{\text {toe }}=0.66 ; x_{\text {toe }}=87.10 ; h_{c}=0.23 ; x_{c}=91.33 ;$

$\mathrm{h}_{\mathrm{t}}=0.22 ; \mathrm{X}_{\mathrm{t}}=99.13 ; \beta_{1}=5 ; \beta_{3}=0$.

$\begin{array}{cccc}\mathrm{H} & \mathrm{X} & \mathrm{h} & \eta \\ 0.34 & 30.00 & 1.25 & -0.010 \\ 0.30 & 85.50 & 0.71 & -0.009 \\ 0.30 & 87.00 & 0.65 & -0.004 \\ 0.33 & 89.42 & 0.39 & -0.013 \\ 0.11 & 92.50 & 0.22 & 0.021 \\ 0.10 & 94.50 & 0.22 & 0.036 \\ 0.08 & 97.00 & 0.21 & 0.042 \\ 0.07 & 100.00 & 0.23 & 0.041\end{array}$

Case 4120: $T=1.02 ; H_{b}=0.32 ; X_{b}=90.93 ; h_{b}=0.29 ; H_{o} / L_{o}=0.070 ;$

$\mathrm{h}_{\text {toe }}=0.58 ; \mathrm{X}_{\text {toe }}=87.10 ; \mathrm{h}_{\mathrm{c}}=0.23 ; \mathrm{x}_{\mathrm{c}}=91.33$;

$\mathrm{h}_{\mathrm{t}}=0.22 ; \mathrm{X}_{\mathrm{t}}=99.13 ; \beta_{1}=10 ; \beta_{3}=0$.

$\mathrm{H} \quad \mathrm{X} \quad \mathrm{h} \quad \eta$

$\begin{array}{lrrr}0.34 & 30.00 & 1.25 & -0.004 \\ 0.31 & 87.46 & 0.64 & -0.003 \\ 0.28 & 89.00 & 0.60 & -0.008 \\ 0.30 & 90.54 & 0.36 & -0.013 \\ 0.19 & 92.50 & 0.21 & 0.021 \\ 0.11 & 94.50 & 0.21 & 0.036 \\ 0.10 & 97.00 & 0.22 & 0.044 \\ 0.08 & 100.00 & 0.24 & 0.042\end{array}$

Case 4130: $T=1.02 ; H_{b}=0.30 ; X_{b}=91.00 ; h_{b}=0.31 ; H_{o} / L_{o}=0.070 ;$

$h_{\text {toe }}=0.57 ; x_{\text {toe }}=90.33 ; h_{c}=0.22 ; x_{c}=91.33 ;$

$h_{\mathrm{t}}=0.21 ; \mathrm{x}_{\mathrm{t}}=99.13 ; \beta_{1}=15 ; \beta_{3}=0$.

$\mathrm{H} \quad \mathrm{X} \quad \mathrm{h} \quad \boldsymbol{\eta}$

$\begin{array}{lrrr}0.34 & 30.00 & 1.25 & -0.020 \\ 0.30 & 88.00 & 0.63 & -0.029 \\ 0.30 & 89.50 & 0.60 & -0.013 \\ 0.30 & 90.83 & 0.22 & -0.018 \\ 0.19 & 92.50 & 0.22 & 0.016 \\ 0.12 & 94.50 & 0.22 & 0.035 \\ 0.10 & 97.00 & 0.21 & 0.043 \\ 0.08 & 100.00 & 0.24 & 0.046\end{array}$


Case 4140: $T=1.01 ; H_{b}=0.30 ; X_{b}=90.68 ; h_{b}=0.46 ; H_{o} / L_{o}=0.070 ;$

$h_{\text {toe }}=0.56 ; x_{\text {toe }}=90.63 ; h_{c}=0.22 ; x_{c}=91.33 ;$

$\mathrm{h}_{\mathrm{t}}=0.21 ; \mathrm{X}_{\mathrm{t}}=99.13 ; \beta_{1}=20 ; \beta_{3}=0$.

$\begin{array}{cccc}\mathrm{H} & \mathrm{X} & \mathrm{h} & \eta \\ 0.34 & 30.00 & 1.25 & -0.006 \\ 0.30 & 88.00 & 0.62 & -0.007 \\ 0.32 & 89.50 & 0.58 & -0.004 \\ 0.31 & 90.92 & 0.37 & -0.012 \\ 0.10 & 93.00 & 0.22 & 0.022 \\ 0.12 & 95.00 & 0.22 & 0.044 \\ 0.09 & 97.00 & 0.20 & 0.051 \\ 0.07 & 100.00 & 0.24 & 0.047\end{array}$

Case 4150: $T=1.00 ; H_{b}=0.00 ; X_{b}=0.00 ; h_{b}=0.00 ; H_{o} / L_{o}=0.070 ;$

$\mathrm{h}_{\text {toe }}=0.53 ; \mathrm{x}_{\text {toe }}=90.63 ; \mathrm{h}_{\mathrm{c}}=0.23 ; \mathrm{x}_{\mathrm{c}}=91.33 ;$

$\mathrm{h}_{\mathrm{t} .}=0.21 ; \mathrm{x}_{\mathrm{t}}=99.13 ; \beta_{1}=30 ; \beta_{3}=0$.

$\mathrm{H} \quad \mathrm{X} \quad \mathrm{h} \quad \eta$

$\begin{array}{llll}0.35 & 30.00 & 1.25 & -0.003\end{array}$

$\begin{array}{lllll}0.30 & 88.50 & 0.61 & -0.008\end{array}$

$\begin{array}{llll}0.31 & 90.00 & 0.57 & -0.004\end{array}$

$\begin{array}{lllll}0.34 & 91.04 & 0.37 & -0.012\end{array}$

$\begin{array}{llll}0.12 & 93.00 & 0.22 & 0.021\end{array}$

$\begin{array}{llll}0.12 & 95.00 & 0.22 & 0.043\end{array}$

$\begin{array}{llll}0.09 & 97.00 & 0.22 & 0.050\end{array}$

$\begin{array}{llll}0.07 & 100.00 & 0.23 & 0.045\end{array}$

Case 4160: T $=1.00 ; H_{b}=0.00 ; X_{b}=0.00 ; h_{b}=0.00 ; H_{o} / L_{o}=0.070 ;$

$h_{\text {toe }}=0.53 ; x_{\text {toe }}=91.00 ; h_{c}=0.22 ; x_{c}=91.33 ;$

$h_{\mathrm{t}}=0.21 ; \mathrm{X}_{\mathrm{t}}=99.13 ; \beta_{1}=40 ; \beta_{3}=0$.

$\mathrm{H}$

$\mathrm{X} \quad \mathrm{h} \quad \eta$

$\begin{array}{llll}0.36 & 30.00 & 1.25 & -0.002\end{array}$

$\begin{array}{llll}0.30 & 88.50 & 0.67 & -0.009\end{array}$

$\begin{array}{llll}0.31 & 90.00 & 0.57 & -0.005\end{array}$

$\begin{array}{llll}0.35 & 91.08 & 0.36 & -0.020\end{array}$

$\begin{array}{llll}0.13 & 93.00 & 0.22 & 0.025\end{array}$

$\begin{array}{llll}0.11 & 95.00 & 0.22 & 0.046\end{array}$

$\begin{array}{llll}0.09 & 97.00 & 0.22 & 0.051\end{array}$

$\begin{array}{llll}0.09 & 100.00 & 0.24 & 0.047\end{array}$ 
Case 4211: $\quad T=1.02 ; H_{b}=0.36 ; X_{b}=87.72 ; h_{b}=0.43 ; H_{o} / L_{o}=0.082 ;$

$h_{\text {toe }}=0.72 ; x_{\text {toe }}=85.29 ; h_{c}=0.29 ; x_{c}=89.38 ;$

$\mathrm{h}_{\mathrm{t}}=0.58 ; \mathrm{x}_{\mathrm{t}}=89.92 ; \beta_{1}=5 ; \beta_{3}=20$.

$\begin{array}{cccc}\mathrm{H} & \mathrm{X} & \mathrm{h} & \eta \\ 0.40 & 30.00 & 1.25 & 0.022 \\ 0.37 & 83.25 & 0.79 & 0.003 \\ 0.35 & 85.00 & 0.73 & -0.006 \\ 0.36 & 87.79 & 0.42 & -0.014 \\ 0.15 & 91.00 & 0.53 & 0.008 \\ 0.18 & 93.00 & 0.46 & 0.022 \\ 0.16 & 96.00 & 0.38 & 0.018 \\ 0.15 & 100.00 & 0.23 & 0.012\end{array}$

Case 4212: $T=1.00 ; H_{b}=0.00 ; X_{b}=0.00 ; h_{b}=0.00 ; H_{o} / L_{o}=0.070 ;$

$h_{\text {toe }}=0.71 ; x_{\text {toe }}=85.38 ; h_{c}=0.25 ; x_{c}=89.46 ;$

$h_{\mathrm{t}}=0.58 ; \mathrm{X}_{\mathrm{t}}=90.00 ; \beta_{1}=5 ; \beta_{3}=20$.

$\begin{array}{llll}\mathrm{H} & \mathrm{X} & \mathrm{h} & \eta\end{array}$

$\begin{array}{llll}0.34 & 30.00 & 1.25 & 0.002 \\ 0.33 & 83.25 & 0.79 & 0.001 \\ 0.31 & 85.00 & 0.73 & 0.001 \\ 0.35 & 87.71 & 0.41 & -0.005 \\ 0.13 & 90.50 & 0.56 & 0.003 \\ 0.17 & 92.50 & 0.47 & 0.011 \\ 0.15 & 95.50 & 0.40 & 0.014 \\ 0.16 & 99.50 & 0.26 & 0.015\end{array}$

Case 4220: $T=1.02 ; H_{b}=0.33 ; X_{b}=90.35 ; h_{b}=0.31 ; H_{o} / L_{o}=0.075 ;$

$\mathrm{h}_{\text {toe }}=0.60 ; \mathrm{x}_{\text {toe }}=89.25 ; \mathrm{h}_{\mathrm{c}}=0.24 ; \mathrm{x}_{\mathrm{c}}=90.75 ;$

$\mathrm{h}_{\mathrm{t}}=0.52 ; \mathrm{X}_{\mathrm{t}}=91.33 ; \beta_{1}=10 ; \beta_{3}=20$.

$\begin{array}{lllll}\mathrm{H} & \mathrm{X} & \mathrm{h} & \eta\end{array}$

$\begin{array}{lrrr}0.37 & 30.00 & 1.25 & -0.008 \\ 0.35 & 87.00 & 0.65 & -0.007 \\ 0.34 & 88.50 & 0.61 & -0.017 \\ 0.38 & 90.29 & 0.32 & -0.015 \\ 0.19 & 92.00 & 0.49 & 0.012 \\ 0.18 & 94.00 & 0.44 & 0.019 \\ 0.18 & 97.00 & 0.34 & 0.019 \\ 0.17 & 100.00 & 0.24 & 0.019\end{array}$


Case 4230: $T=1.02 ; H_{b}=0.32 ; X_{b}=90.59 ; h_{b}=0.35 ; H_{o} / L_{o}=0.075 ;$

$h_{\text {toe }}=0.57 ; x_{\text {toe }}=90.08 ; h_{c}=0.24 ; x_{c}=91.00$;

$h_{t}=0.51 ; X_{t}=91.58 ; \beta_{1}=15 ; \beta_{3}=20$.

$\mathrm{H}$

$\mathrm{X}$

$\mathrm{h}$

$\eta$

$\begin{array}{lrrr}0.37 & 30.00 & 1.25 & -0.003 \\ 0.32 & 87.50 & 0.64 & -0.012 \\ 0.36 & 89.00 & 0.60 & -0.015 \\ 0.42 & 90.71 & 0.32 & -0.015 \\ 0.15 & 92.50 & 0.47 & 0.008 \\ 0.18 & 94.50 & 0.43 & 0.017 \\ 0.17 & 97.50 & 0.32 & 0.019 \\ 0.16 & 100.50 & 0.22 & 0.021\end{array}$

Case 4240: $T=1.02 ; H_{b}=0.35 ; X_{b}=90.33 ; h_{b}=0.54 ; H_{o} / L_{o}=0.076 ;$

$h_{\text {toe }}=0.56 ; x_{\text {toe }}=90.33 ; h_{c}=0.24 ; x_{c}=91.00 ;$

$h_{\mathrm{t}}=0.51 ; \mathrm{X}_{\mathrm{t}}=91.58 ; \beta_{1}=20 ; \beta_{3}=20$.

$\mathrm{H}$

$\mathrm{X}$

$\mathrm{h}$

$\eta$

$\begin{array}{llll}0.38 & 30.00 & 1.25 & 0.000\end{array}$

$\begin{array}{llll}0.32 & 87.50 & 0.64 & -0.012\end{array}$

$\begin{array}{llll}0.37 & 89.00 \quad 0.60 & -0.010\end{array}$

$\begin{array}{llll}0.42 & 90.63 & 0.35 & -0.013\end{array}$

$\begin{array}{llll}0.16 & 92.50 & 0.47 & 0.006\end{array}$

$\begin{array}{llll}0.18 & 94.50 & 0.43 & 0.017\end{array}$

$\begin{array}{llll}0.17 & 97.50 & 0.32 & 0.022\end{array}$

$\begin{array}{llll}0.15 & 100.50 & 0.22 & 0.025\end{array}$

Case 4250: $T=1.00 ; H_{b}=0.00 ; X_{b}=0.00 ; h_{b}=0.00 ; H_{o} / L_{o}=0.070 ;$

$h_{\text {toe }}=0.57 ; x_{\text {toe }}=90.33 ; h_{c}=0.24 ; x_{c}=90.75 ;$

$h_{\mathrm{t}}=0.52 ; \mathrm{X}_{\mathrm{t}}=91.33 ; \beta_{1}=30 ; \beta_{3}=20$.

$\begin{array}{lccc}\mathrm{H} & \mathrm{X} & \mathrm{h} & \eta \\ 0.38 & 30.00 & 1.25 & 0.007 \\ 0.29 & 87.50 & 0.64 & -0.011 \\ 0.37 & 88.33 & 0.62 & -0.004 \\ 0.45 & 90.50 & 0.33 & -0.013 \\ 0.18 & 92.50 & 0.47 & 0.014 \\ 0.20 & 94.50 & 0.43 & 0.019 \\ 0.16 & 97.50 & 0.33 & 0.025 \\ 0.14 & 100.50 & 0.22 & 0.028\end{array}$


Case 4260: $T=1.00 ; H_{b}=0.00 ; X_{b}=0.00 ; h_{b}=0.00 ; H_{o} / L_{o}=0.070 ;$

$h_{\text {toe }}=0.55 ; x_{\text {toe }}=90.67 ; h_{c}=0.24 ; x_{c}=91.00 ;$

$h_{\mathrm{t}}=0.51 ; \mathrm{X}_{\mathrm{t}}=91.58 ; \beta_{1}=40 ; \beta_{3}=20$.

$\begin{array}{cccc}\mathrm{H} & \mathrm{X} & \mathrm{h} & \eta \\ 0.34 & 30.00 & 1.25 & -0.001 \\ 0.26 & 87.96 & 0.63 & -0.013 \\ 0.29 & 89.46 & 0.59 & -0.007 \\ 0.38 & 90.75 & 0.38 & -0.018 \\ 0.18 & 92.46 & 0.47 & 0.004 \\ 0.20 & 94.00 & 0.44 & 0.009 \\ 0.19 & 96.00 & 0.38 & 0.015 \\ 0.14 & 100.00 & 0.23 & 0.025\end{array}$

Case 4310: $T=1.02 ; H_{b}=0.31 ; X_{b}=88.14 ; h_{b}=0.44 ; H_{o} / L_{o}=0.069 ;$

$\mathrm{h}_{\text {toe }}=0.68 ; \mathrm{x}_{\text {toe }}=86.08 ; \mathrm{h}_{\mathrm{c}}=0.28 ; \mathrm{x}_{\mathrm{c}}=90.00$;

$\mathrm{h}_{\mathrm{t}}=0.56 ; \mathrm{x}_{\mathrm{t}}=90.38 ; \beta_{1}=5 ; \beta_{3}=30$.

$\mathrm{H} \quad \mathrm{X}$ h $\eta$

$\begin{array}{llll}0.34 & 30.00 & 1.25 & -0.005\end{array}$

$\begin{array}{llll}0.30 & 84.50 & 0.75 & -0.004\end{array}$

$\begin{array}{llll}0.33 & 86.00 & 0.68 & 0.002\end{array}$

$\begin{array}{llll}0.34 & 88.25 & 0.42 & -0.009\end{array}$

$\begin{array}{llll}0.15 & 91.25 & 0.51 & 0.005\end{array}$

$\begin{array}{llll}0.17 & 93.33 & 0.46 & 0.011\end{array}$

$\begin{array}{lllll}0.16 & 96.33 & 0.37 & 0.010\end{array}$

$\begin{array}{llll}0.17 & 99.83 & 0.25 & 0.013\end{array}$

Case 4320: $T=1.02 ; H_{b}=0.32 ; X_{b}=90.78 ; h_{b}=0.32 ; H_{o} / L_{o}=0.070 ;$

$h_{\text {toe }}=0.58 ; X_{\text {toe }}=89.83 ; h_{c}=0.23 ; X_{c}=91.33$;

$h_{\mathrm{t}}=0.50 ; \mathrm{X}_{\mathrm{t}}=91.71 ; \beta_{1}=10 ; \beta_{3}=30$.

$\mathrm{H}$

$\mathrm{X}$

$\mathrm{h}$

$\eta$

0.34

30.00

1.25

$-0.005$

0.30

88.04

0.62

$-0.011$

0.33

89.50

0.58

$-0.003$

0.37

90.88

0.31

$-0.010$

0.15

92.67

0.47

0.004

0.18

94.67

0.43

0.015

0.17

96.67

0.35

0.014

0.18

100.00

0.24

0.017 
Case 4330: $T=1.02 ; H_{b}=0.29 ; X_{b}=91.53 ; h_{b}=0.35 ; H_{o} / L_{o}=0.069 ;$

$h_{\text {toe }}=0.63 ; x_{\text {toe }}=91.08 ; h_{c}=0.23 ; x_{c}=92.00 ;$

$h_{\mathrm{t}}=0.48 ; \mathrm{x}_{\mathrm{t}}=92.75 ; \beta_{1}=15 ; \beta_{3}=30$.

$\begin{array}{cccc}\text { H } & \text { X } & \text { h } & \eta \\ 0.34 & 30.00 & 1.25 & -0.004 \\ 0.29 & 88.96 & 0.60 & -0.014 \\ 0.27 & 90.50 & 0.55 & -0.010 \\ 0.38 & 91.54 & 0.31 & -0.011 \\ 0.12 & 93.46 & 0.45 & 0.002 \\ 0.17 & 95.50 & 0.39 & 0.010 \\ 0.16 & 97.50 & 0.32 & 0.015 \\ 0.15 & 100.50 & 0.22 & 0.018\end{array}$

Case 4340: $T=1.02 ; H_{b}=0.30 ; X_{b}=91.45 ; h_{b}=0.44 ; H_{o} / L_{o}=0.068 ;$

$h_{\text {toe }}=0.51 ; x_{\text {toe }}=91.33 ; h_{c}=0.23 ; x_{c}=92.04 ;$

$h_{t}=0.48 ; x_{t}=92.42 ; \beta_{1}=20 ; \beta_{3}=30$.

$\mathrm{H} \quad \mathrm{X} \quad \mathrm{h} \quad \eta$

$\begin{array}{lrrr}0.34 & 30.00 & 1.25 & -0.001 \\ 0.28 & 89.00 & 0.60 & -0.012 \\ 0.29 & 90.50 & 0.55 & -0.005 \\ 0.38 & 91.67 & 0.32 & -0.008 \\ 0.14 & 93.50 & 0.45 & -0.001 \\ 0.17 & 95.50 & 0.39 & 0.012 \\ 0.16 & 97.50 & 0.32 & 0.014 \\ 0.15 & 100.50 & 0.22 & 0.020\end{array}$

Case 4350: $T=1.00 ; H_{b}=0.00 ; X_{b}=0.00 ; h_{b}=0.00 ; H_{o} / L_{o}=0.070 ;$

$\mathrm{h}_{\text {toe }}=0.53 ; \mathrm{x}_{\text {toe }}=91.13 ; \mathrm{h}_{\mathrm{c}}=0.23 ; \mathrm{x}_{\mathrm{c}}=91.54 ;$

$h_{t}=0.50 ; x_{t}=91.92 ; \beta_{1}=30 ; \beta_{3}=30$.

$\begin{array}{cccc}\text { H } & \mathrm{X} & \mathrm{h} & \eta \\ 0.34 & 30.00 & 1.25 & -0.001 \\ 0.26 & 88.50 & -0.61 & -0.014 \\ 0.29 & 90.00 & 0.57 & -0.013 \\ 0.39 & 91.33 & 0.30 & -0.020 \\ 0.17 & 93.00 & 0.46 & 0.004 \\ 0.18 & 95.00 & 0.41 & 0.012 \\ 0.16 & 97.00 & 0.33 & 0.015 \\ 0.15 & 100.00 & 0.23 & 0.020\end{array}$


Case 4360: $T=1.00 ; H_{b}=0.00 ; X_{b}=0.00 ; h_{b}=0.00 ; H_{o} / L_{o}=0.070 ;$

$\mathrm{h}_{\text {toe }}=0.51 ; \mathrm{x}_{\mathrm{toe}}=91.42 ; \mathrm{h}_{\mathrm{c}}=0.23 ; \mathrm{x}_{\mathrm{c}}=91.67 ;$

$h_{\mathrm{t}}=0.49 ; \mathrm{x}_{\mathrm{t}}=92.08 ; \beta_{1}=40 ; \beta_{3}=30$.

$\begin{array}{lccc}\mathrm{H} & \mathrm{X} & \mathrm{h} & \eta \\ 0.33 & 30.00 & 1.25 & -0.007 \\ 0.28 & 88.75 & 0.61 & -0.013 \\ 0.28 & 90.25 & 0.56 & -0.014 \\ 0.39 & 91.46 & 0.37 & -0.023 \\ 0.18 & 93.00 & 0.46 & 0.004 \\ 0.19 & 95.00 & 0.41 & 0.010 \\ 0.17 & 97.00 & 0.33 & 0.017 \\ 0.14 & 100.00 & 0.24 & 0.023\end{array}$

Case 4410: $T=1.02 ; H_{b}=0.30 ; X_{b}=89.39 ; h_{b}=0.37 ; H_{o} / L_{o}=0.069 ;$

$h_{\text {toe }}=0.66 ; X_{\text {toe }}=87.04 ; h_{c}=0.24 ; X_{c}=90.96 ;$

$\mathrm{h}_{\mathrm{t}}=0.52 ; \mathrm{x}_{\mathrm{t}}=91.17 ; \beta_{1}=5 ; \beta_{3}=40$.

$\mathrm{H}$ $\mathrm{X}$

h

0.34

30.00

1.25

$\eta$

0.32

85.33

0.71

$-0.002$

0.33

86.83

0.65

$-0.007$

0.36

89.00

0.41

$-0.001$

0.12

92.50

0.47

$-0.011$

0.17

94.75

0.42

0.011

0.15

97.00

0.33

0.016

0.17

100.00

0.23

0.016

0.018

Case 4420: $T=1.02 ; H_{b}=0.33 ; X_{b}=90.35 ; h_{b}=0.31 ; H_{o} / L_{o}=0.075 ;$

$h_{\text {toe }}=0.57 ; x_{\text {toe }}=90.21 ; h_{c}=0.21 ; x_{c}=91.71 ;$

$h_{t}=0.50 ; X_{t}=91.96 ; \beta_{1}=10 ; \beta_{3}=40$.

$\mathrm{H}$ $\mathrm{X}$

0.34

30.00

h

$\eta$

0.31

88.25

1. 25

$-0.005$

0.35

89.75

0.62

$-0.010$

0.37

91.04

0.58

$-0.001$

0.16

93.00

0.31

$-0.010$

0.17

95.00

0.46

0.004

0.17

97.00

0.40

0.015

0.17

100.00

0.33

0.017

0.23

0.018 
Case 4430: $T=1.02 ; H_{b}=0.30 ; X_{b}=91.11 ; h_{b}=0.40 ; H_{o} / L_{o}=0.069 ;$

$h_{\text {toe }}=0.54 ; x_{\text {toe }}=90.79 ; h_{c}=0.23 ; x_{c}=91.75 ;$

$h_{\mathrm{t}}=0.49 ; \mathrm{x}_{\mathrm{t}}=92.00 ; \beta_{1}=15 ; \beta_{3}=40$.

$\begin{array}{cccc}\mathrm{H} & \mathrm{X} & \mathrm{h} & \eta \\ 0.34 & 30.00 & 1.25 & -0.005 \\ 0.29 & 88.50 & 0.61 & -0.009 \\ 0.30 & 90.00 & 0.56 & -0.008 \\ 0.40 & 91.29 & 0.31 & -0.014 \\ 0.15 & 93.00 & 0.46 & 0.003 \\ 0.20 & 95.00 & 0.41 & 0.011 \\ 0.17 & 97.00 & 0.32 & 0.016 \\ 0.16 & 100.00 & 0.23 & 0.018\end{array}$

Case 4440: $T=1.02 ; H_{b}=0.30 ; X_{b}=90.83 ; h_{b}=0.51 ; H_{o} / L_{o}=0.069 ;$

$h_{\text {toe }}=0.53 ; x_{\text {toe }}=90.83 ; h_{c}=0.22 ; x_{c}=91.54 ;$

$\mathrm{h}_{\mathrm{t}}=0.50 ; \mathrm{X}_{\mathrm{t}}=91.79 ; \beta_{1}=20 ; \beta_{3}=40$.

$\begin{array}{cccc}\mathrm{H} & \mathrm{X} & \mathrm{h} & \eta \\ 0.34 & 30.00 & 1.25 & -0.002 \\ 0.29 & 88.50 & 0.60 & -0.010 \\ 0.29 & 90.00 & 0.56 & -0.012 \\ 0.40 & 91.13 & 0.33 & -0.010 \\ 0.18 & 93.00 & 0.46 & 0.002 \\ 0.20 & 95.00 & 0.41 & 0.012 \\ 0.19 & 97.00 & 0.32 & 0.013 \\ 0.17 & 100.00 & 0.23 & 0.017\end{array}$

Case 4450: $T=1.00 ; H_{b}=0.00 ; X_{b}=0.00 ; h_{b}=0.00 ; H_{o} / L_{o}=0.070 ;$

$h_{\text {toe }}=0.52 ; x_{\text {toe }}=91.29 ; h_{c}=0.23 ; x_{c}=91.75 ;$

$h_{\mathrm{t}}=0.49 ; \mathrm{X}_{\mathrm{t}}=92.00 ; \beta_{1}=30 ; \beta_{3}=40$.

$\mathrm{H}$

$\mathrm{X}$

$\mathrm{h}$

$\eta$

0.34

30.00

1.25

$-0.009$

0.29

88.75

0.60

$-0.014$

0.29

90.25

0.56

$-0.013$

0.41

91.42

0.23

$-0.017$

0.18

93.00

0.46

0.003

0.18

95.00

0.41

0.012

0.17

97.00

0.32

0.015

0.15

100.00

0.23

0.022 
Case 4460: $T=1.00 ; H_{b}=0.00 ; X_{b}=0.00 ; h_{b}=0.00 ; H_{o} / L_{o}=0.070 ;$

$h_{\text {toe }}=0.52 ; x_{\text {toe }}=91.33 ; h_{c}=0.22 ; x_{c}=91.67 ;$

$h_{t}=0.50 ; X_{t}=91.96 ; \beta_{1}=40 ; \beta_{3}=40$.

$\begin{array}{cccc}\mathrm{H} & \mathrm{X} & \mathrm{h} & \eta \\ 0.34 & 30.00 & 1.25 & -0.002 \\ 0.29 & 88.75 & 0.60 & -0.013 \\ 0.29 & 90.25 & 0.57 & -0.013 \\ 0.41 & 91.46 & 0.35 & -0.026 \\ 0.17 & 93.00 & 0.46 & 0.002 \\ 0.19 & 95.00 & 0.41 & 0.011 \\ 0.17 & 97.00 & 0.33 & 0.018 \\ 0.14 & 100.00 & 0.23 & 0.022\end{array}$

Case 6111: $T=1.49 ; H_{b}=0.51 ; X_{b}=81.60 ; h_{b}=0.60 ; H_{o} / L_{o}=0.046 ;$

$h_{\text {toe }}=1.00 ; x_{\text {toe }}=78.13 ; h_{c}=0.29 ; x_{c}=85.17$;

$h_{t}=0.32 ; X_{t}=95.63 ; \beta_{1}=5 ; \beta_{3}=0$.

$\begin{array}{cccc}\mathrm{H} & \mathrm{X} & \mathrm{h} & \eta \\ 0.48 & 30.00 & 1.25 & -0.012 \\ 0.48 & 75.00 & 1.05 & -0.010 \\ 0.47 & 77.95 & 0.96 & -0.011 \\ 0.52 & 81.83 & 0.61 & -0.020 \\ 0.17 & 86.50 & 0.32 & 0.018 \\ 0.15 & 89.50 & 0.31 & 0.035 \\ 0.12 & 93.50 & 0.31 & 0.041 \\ 0.14 & 98.50 & 0.30 & 0.040\end{array}$

Case 6112: $T=1.50 ; \mathrm{H}_{\mathrm{b}}=0.00 ; \mathrm{X}_{\mathrm{b}}=0.00 ; \mathrm{h}_{\mathrm{b}}=0.00 ; \mathrm{H}_{\mathrm{o}} / \mathrm{L}_{\mathrm{o}}=0.050 ;$

$h_{\text {toe }}=1.06 ; x_{\text {toe }}=74.83 ; h_{c}=0.42 ; x_{c}=81.83$;

$h_{t}=0.46 ; x_{t}=92.30 ; \beta_{1}=5 ; \beta_{3}=0$.

H

$\mathrm{X}$

h

$\eta$

$\begin{array}{llll}0.50 & 30.00 & 1.25 & -0.014\end{array}$

$\begin{array}{llll}0.47 & 70.92 & 1.20 & -0.014\end{array}$

$\begin{array}{llll}0.59 & 74.50 & 1.07 & -0.010\end{array}$

$\begin{array}{llll}0.53 & 79.33 & 0.63 & -0.022\end{array}$

$\begin{array}{llll}0.16 & 84.00 & 0.42 & 0.041\end{array}$

$\begin{array}{llll}0.14 & 88.00 & 0.42 & 0.059\end{array}$

$\begin{array}{llll}0.11 & 93.00 & 0.44 & 0.064\end{array}$

$\begin{array}{llll}0.10 & 98.00 & 0.31 & 0.063\end{array}$ 
Case 6120: $T=1.49 ; H_{b}=0.63 ; X_{b}=84.17 ; h_{b}=0.47 ; H_{o} / L_{o}=0.048 ;$

$\mathrm{h}_{\text {toe }}=0.81 ; \mathrm{x}_{\text {toe }}=82.48 ; \mathrm{h}_{\mathrm{c}}=0.30 ; \mathrm{x}_{\mathrm{c}}=85.17$;

$h_{\mathrm{t}}=0.33 ; \mathrm{x}_{\mathrm{t}}=95.63 ; \beta_{1}=10 ; \beta_{3}=0$.

$\mathrm{H}$ $\mathrm{X}$

$\mathrm{h}$

$\eta$

0.51

30.00

1.25

$-0.019$

0.51

79.50

0.91

$-0.017$

0.67

82.00

0.81

0.020

0.61

84.00

0.50

$-0.026$

0.27

86.50

0.29

0.042

0.15

89.50

0.31

0.069

0.11

93.50

0.32

0.076

0.11

98.50

0.31

0.078

Case 6130: $T=1.50 ; \mathrm{H}_{\mathrm{b}}=0.62 ; \mathrm{X}_{\mathrm{b}}=84.33 ; \mathrm{h}_{\mathrm{b}}=0.51 ; \mathrm{H}_{\mathrm{o}} / \mathrm{L}_{\mathrm{o}}=0.052 ;$

$h_{\text {toe }}=0.79 ; x_{\text {toe }}=83.54 ; h_{c}=0.29 ; x_{c}=85.17$;

$h_{\mathrm{t}}=0.33 ; \mathrm{X}_{\mathrm{t}}=95.63 ; \beta_{1}=15 ; \beta_{3}=0$.

$\mathrm{H}$ $\mathrm{X}$

h

$\eta$

$\begin{array}{lllr}0.51 & 30.00 & 1.25 & -0.021 \\ 0.56 & 81.00 & 0.85 & -0.015 \\ 0.63 & 83.00 & 0.79 & 0.023 \\ 0.58 & 84.30 & 0.50 & -0.029 \\ 0.25 & 86.50 & 0.30 & 0.038 \\ 0.17 & 89.50 & 0.31 & 0.070 \\ 0.11 & 93.50 & 0.32 & 0.082 \\ 0.10 & 98.50 & 0.30 & 0.083\end{array}$

Case 6140: $T=1.50 ; H_{b}=0.71 ; X_{b}=84.27 ; h_{b}=0.63 ; H_{o} / L_{o}=0.048 ;$

$h_{\text {toe }}=0.76 ; x_{\text {toe }}=83.96 ; h_{c}=0.30 ; x_{c}=85.17 ;$

$h_{\mathrm{t}}=0.32 ; \mathrm{x}_{\mathrm{t}}=95.63 ; \beta_{1}=20 ; \beta_{3}=0$.

$\mathrm{H}$

$\mathrm{X}$

$\mathrm{h}$

$\eta$

$\begin{array}{lllr}0.51 & 30.00 & 1.25 & -0.021 \\ 0.58 & 81.20 & 0.85 & -0.012 \\ 0.63 & 83.20 & 0.79 & 0.008 \\ 0.55 & 84.38 & 0.57 & -0.022 \\ 0.22 & 86.50 & 0.30 & 0.033 \\ 0.18 & 89.50 & 0.31 & 0.076 \\ 0.12 & 93.50 & 0.32 & 0.085 \\ 0.10 & 98.50 & 0.31 & 0.086\end{array}$


Case 6210: $T=1.49 ; H_{b}=0.46 ; X_{b}=80.19 ; h_{b}=0.63 ; H_{o} / L_{o}=0.048 ;$

$\mathrm{h}_{\text {toe }}=1.00 ; \mathrm{X}_{\text {toe }}=78.83 ; \mathrm{h}_{\mathrm{c}}=0.38 ; \mathrm{X}_{\mathrm{c}}=83.08 ;$

$\mathrm{h}_{\mathrm{t}}=0.76 ; \mathrm{x}_{\mathrm{t}}=84.00 ; \beta_{1}=5 ; \beta_{3}=20$.

$\begin{array}{cccc}\text { H } & \text { X } & \text { h } & \eta \\ 0.51 & 30.00 & 1.25 & -0.002 \\ 0.43 & 73.50 & 1.10 & -0.007 \\ 0.66 & 77.50 & 1.01 & 0.037 \\ 0.43 & 82.46 & 0.44 & -0.016 \\ 0.21 & 85.00 & 0.78 & 0.002 \\ 0.28 & 89.00 & 0.60 & 0.018 \\ 0.25 & 94.00 & 0.44 & 0.018 \\ 0.27 & 98.67 & 0.28 & 0.021\end{array}$

Case 6220: $T=1.49 ; \mathrm{H}_{\mathrm{b}}=0.57 ; \mathrm{X}_{\mathrm{b}}=84.48 ; \mathrm{h}_{\mathrm{b}}=0.45 ; \mathrm{H}_{\mathrm{o}} / \mathrm{L}_{\mathrm{o}}=0.043 ;$

$\mathrm{h}_{\text {toe }}=0.81 ; \mathrm{x}_{\text {toe }}=82.08 ; \mathrm{h}_{\mathrm{c}}=0.33 ; \mathrm{x}_{\mathrm{c}}=85.21$;

$\mathrm{h}_{\mathrm{t}}=0.68 ; \mathrm{x}_{\mathrm{t}}=86.08 ; \beta_{1}=10 ; \beta_{3}=20$.

$\mathrm{H}$

$\mathrm{X}$

h

$\eta$

$\begin{array}{llll}0.46 & 30.00 & 1.25 & -0.005 \\ 0.55 & 80.46 & 0.87 & -0.010 \\ 0.62 & 82.46 & 0.81 & 0.034 \\ 0.58 & 84.46 & 0.45 & -0.020 \\ 0.22 & 87.00 & 0.65 & 0.003 \\ 0.23 & 90.00 & 0.57 & 0.016 \\ 0.25 & 94.00 & 0.45 & 0.023 \\ 0.19 & 98.67 & 0.29 & 0.030\end{array}$

Case 6230: $T=1.49 ; H_{b}=0.63 ; X_{b}=85.15 ; h_{b}=0.52 ; H_{o} / L_{o}=0.042 ;$

$\mathrm{h}_{\text {toe }}=0.75 ; \mathrm{x}_{\text {toe }}=84.42 ; \mathrm{h}_{\mathrm{c}}=0.33 ; \mathrm{x}_{\mathrm{c}}=85.88$;

$h_{\mathrm{t}}=0.67 ; \mathrm{x}_{\mathrm{t}}=86.75 ; \beta_{1}=15 ; \beta_{3}=20$.

$\mathrm{H}$

$\mathrm{X}$

$\mathrm{h}$

$\eta$

0.44

31.00

1.25

$-0.008$

0.58

81.25

0.85

$-0.006$

0.56

84.00

0.76

0.048

0.55

85.08

0.51

$-0.016$

0.21

88.00

0.63

$-0.001$

0.24

91.00

0.54

0.018

0.23

94.50

0.44

0.025

0.17

98.50

0.29

0.032 
Case 6240: $T=1.50 ; H_{b}=0.60 ; X_{b}=83.75 ; h_{b}=0.77 ; H_{o} / L_{o}=0.043 ;$

$\mathrm{h}_{\text {toe }}=0.77 ; \mathrm{x}_{\text {toe }}=83.75 ; \mathrm{h}_{\mathrm{c}}=0.31 ; \mathrm{x}_{\mathrm{c}}=84.83 ;$

$\mathrm{h}_{\mathrm{t}}=0.70 ; \mathrm{x}_{\mathrm{t}}=85.67 ; \beta_{1}=20 ; \beta_{3}=20$.

$\begin{array}{cccc}\text { H } & \mathrm{X} & \mathrm{h} & \eta \\ 0.46 & 30.00 & 1.25 & -0.005 \\ 0.59 & 80.50 & 0.87 & -0.009 \\ 0.61 & 83.00 & 0.79 & 0.036 \\ 0.58 & 84.21 & 0.50 & -0.016 \\ 0.27 & 86.50 & 0.62 & 0.001 \\ 0.29 & 89.50 & 0.59 & 0.018 \\ 0.24 & 93.50 & 0.45 & 0.026 \\ 0.17 & 98.50 & 0.29 & 0.037\end{array}$

Case 6250: $T=1.50 ; H_{b}=0.00 ; X_{b}=0.00 ; h_{b}=0.00 ; H_{o} / L_{o}=0.050 ;$

$\mathrm{h}_{\text {toe }}=0.75 ; \mathrm{x}_{\text {toe }}=94.25 ; \mathrm{h}_{\mathrm{c}}=0.32 ; \mathrm{x}_{\mathrm{c}}=94.87$;

$\mathrm{h}_{\mathrm{t}}=0.69 ; \mathrm{x}_{\mathrm{t}}=95.75 ; \beta_{1}=30 ; \beta_{3}=20$.

$\mathrm{H} \quad \mathrm{X} \quad \mathrm{h} \quad \eta$

$\begin{array}{lllr}0.44 & 30.00 & 1.25 & -0.006 \\ 0.60 & 80.50 & 0.86 & -0.009 \\ 0.59 & 83.00 & 0.79 & 0.036 \\ 0.59 & 84.38 & 0.58 & -0.015 \\ 0.27 & 86.50 & 0.65 & 0.003 \\ 0.30 & 89.50 & 0.58 & 0.022 \\ 0.22 & 93.50 & 0.45 & 0.031 \\ 0.14 & 98.50 & 0.29 & 0.043\end{array}$

Case 6310: $T=1.47 ; \mathrm{H}_{\mathrm{b}}=0.56 ; \mathrm{X}_{\mathrm{b}}=79.91 ; \mathrm{h}_{\mathrm{b}}=0.63 ; \mathrm{H}_{\mathrm{o}} / \mathrm{L}_{\mathrm{o}}=0.048 ;$

$h_{\text {toe }}=1.02 ; x_{\text {toe }}=76.75 ; h_{c}=0.38 ; x_{c}=82.83 ;$

$h_{t}=0.78 ; X_{t}=83.42 ; \beta_{1}=5 ; \beta_{3}=30$.

$\mathrm{H}$ $\mathrm{X} \quad \mathrm{h}$

$0.49 \quad 30.00$

1.26

$-0.004$

0.47

73.46

1.11

$-0.004$

0.48

76.46

1.02

$-0.012$

0.51

80.92

0.55

$-0.016$

0.21

85.96

0.68

0.013

0.27

89.50

0.59

0.019

0.27

93.50

0.46

0.021

0.31

97.50

0.32

0.022 
Case 6320: $T=1.49 ; \mathrm{H}_{\mathrm{b}}=0.60 ; \mathrm{X}_{\mathrm{b}}=84.43 ; \mathrm{h}_{\mathrm{b}}=0.45 ; \mathrm{H}_{\mathrm{o}} / \mathrm{L}_{\mathrm{o}}=0.043 ;$

$\mathrm{h}_{\text {toe }}=0.79 ; \mathrm{x}_{\text {toe }}=82.83 ; \mathrm{h}_{\mathrm{c}}=0.32 ; \mathrm{x}_{\mathrm{c}}=85.19 ;$

$h_{\mathrm{t}}=0.69 ; \mathrm{x}_{\mathrm{t}}=85.75 ; \beta_{1}=10 ; \beta_{3}=30$.

$\begin{array}{cccc}\mathrm{H} & \mathrm{X} & \mathrm{h} & \eta \\ 0.46 & 30.00 & 1.25 & -0.009 \\ 0.56 & 80.75 & 0.85 & -0.010 \\ 0.71 & 82.75 & 0.79 & 0.016 \\ 0.58 & 84.38 & 0.45 & -0.018 \\ 0.18 & 87.25 & 0.65 & 0.007 \\ 0.26 & 89.75 & 0.58 & 0.017 \\ 0.25 & 93.50 & 0.46 & 0.022 \\ 0.18 & 97.50 & 0.32 & 0.029\end{array}$

Case 6330: $T=1.50 ; \mathrm{H}_{\mathrm{b}}=0.57 ; \mathrm{X}_{\mathrm{b}}=84.13 ; \mathrm{h}_{\mathrm{b}}=0.58 ; \mathrm{H}_{\mathrm{o}} / \mathrm{L}_{\mathrm{o}}=0.043 ;$

$\mathrm{h}_{\text {toe }}=0.72 ; \mathrm{x}_{\text {toe }}=83.67 ; \mathrm{h}_{\mathrm{c}}=0.32 ; \mathrm{x}_{\mathrm{c}}=85.10$;

$h_{\mathrm{t}}=0.70 ; \mathrm{x}_{\mathrm{t}}=85.67 ; \beta_{1}=15 ; \beta_{3}=30$.

$\mathrm{H} \quad \mathrm{X} \quad \mathrm{h} \quad \eta$

$\begin{array}{llll}0.45 & 30.00 & 1.25 & -0.007\end{array}$

$\begin{array}{llll}0.59 & 80.75 & 0.86 & -0.010\end{array}$

$\begin{array}{llll}0.71 & 83.25 & 0.79 & 0.016\end{array}$

$\begin{array}{llll}0.59 & 84.67 & 0.51 & -0.020\end{array}$

$\begin{array}{llll}0.21 & 87.25 & 0.66 & 0.000\end{array}$

$\begin{array}{llll}0.27 & 89.75 & 0.58 & 0.012\end{array}$

$\begin{array}{llll}0.23 & 93.50 & 0.46 & 0.020\end{array}$

$\begin{array}{llll}0.19 & 97.50 & 0.32 & 0.031\end{array}$

Case 6340: $T=1.50 ; H_{b}=0.57 ; X_{b}=84.08 ; h_{b}=0.75 ; H_{o} / L_{o}=0.042 ;$

$\mathrm{h}_{\text {toe }}=0.76 ; \mathrm{x}_{\text {toe }}=84.08 ; \mathrm{h}_{\mathrm{c}}=0.32 ; \mathrm{X}_{\mathrm{c}}=85.08 ;$

$\mathrm{h}_{\mathrm{t}}=0.70 ; \mathrm{x}_{\mathrm{t}}=85.67 ; \beta_{1}=20 ; \beta_{3}=30$.

$\mathrm{H} \quad \mathrm{X} \quad \mathrm{h} \quad \boldsymbol{H}$

$\begin{array}{llll}0.45 & 30.00 & 1.25 & -0.007\end{array}$

$\begin{array}{llll}0.60 & 81.00 \quad 0.85 & -0.008\end{array}$

$\begin{array}{llll}0.73 & 83.50 & 0.78 & 0.014\end{array}$

$\begin{array}{llll}0.62 & 84.67 & 0.44 & -0.038\end{array}$

$\begin{array}{llll}0.24 & 87.25 & 0.64 & -0.004\end{array}$

$\begin{array}{llll}0.28 & 89.75 & 0.58 & 0.012\end{array}$

$\begin{array}{llll}0.24 & 93.50 & 0.46 & 0.022\end{array}$

$\begin{array}{llll}0.15 & 97.50 & 0.33 & 0.032\end{array}$ 
Case 6410: $T=1.49 ; H_{b}=0.51 ; X_{b}=80.72 ; h_{b}=0.56 ; H_{o} / L_{o}=0.046 ;$

$\mathrm{h}_{\text {toe }}=1.15 ; \mathrm{x}_{\text {toe }}=76.67 ; \mathrm{h}_{\mathrm{c}}=0.39 ; \mathrm{x}_{\mathrm{c}}=82.67 ;$

$h_{\mathrm{t}}=0.79 ; \mathrm{X}_{\mathrm{t}}=83.08 ; \beta_{1}=5 ; \beta_{3}=40$.

$\begin{array}{lllll}\mathrm{H} & \mathrm{X} & \mathrm{h} & \boldsymbol{\eta}\end{array}$

$\begin{array}{llll}0.49 & 30.00 & 1.26 & -0.006\end{array}$

$\begin{array}{llll}0.48 & 73.50 & 1.12 & -0.006\end{array}$

$\begin{array}{llll}0.69 & 76.50 & 1.15 & 0.018\end{array}$

$\begin{array}{llll}0.54 & 81.33 & 0.52 & -0.011\end{array}$

$\begin{array}{llll}0.25 & 84.50 & 0.75 & 0.002\end{array}$

$\begin{array}{llll}0.28 & 87.50 & 0.64 & 0.012\end{array}$

$\begin{array}{llll}0.28 & 92.50 \quad 0.47 & 0.017\end{array}$

$\begin{array}{llll}0.27 & 97.50 & 0.32 & 0.018\end{array}$

Case 6420: $T=1.49 ; H_{b}=0.54 ; X_{b}=84.46 ; h_{b}=0.45 ; H_{o} / L_{o}=0.043 ;$

$\mathrm{h}_{\text {toe }}=0.79 ; \mathrm{x}_{\text {toe }}=83.00 ; \mathrm{h}_{\mathrm{c}}=0.30 ; \mathrm{x}_{\mathrm{c}}=85.31$;

$h_{\mathrm{t}}=0.70 ; \mathrm{X}_{\mathrm{t}}=85.71 ; \beta_{1}=10 ; \beta_{3}=40$.

$\begin{array}{llll}\mathrm{H} & \mathrm{X} & \mathrm{h} & \eta\end{array}$

$\begin{array}{lllr}0.46 & 30.00 & 1.26 & -0.007 \\ 0.56 & 80.25 & 0.88 & -0.013 \\ 0.62 & 82.75 & 0.80 & 0.036 \\ 0.60 & 84.46 & 0.44 & -0.021 \\ 0.21 & 86.75 & 0.65 & -0.001 \\ 0.25 & 88.75 & 0.61 & 0.014 \\ 0.25 & 93.00 & 0.46 & 0.025 \\ 0.19 & 98.00 & 0.31 & 0.033\end{array}$

Case 6430: $T=1.50 ; H_{b}=0.59 ; X_{b}=84.46 ; h_{b}=0.53 ; H_{o} / L_{o}=0.043 ;$

$h_{\text {toe }}=0.77 ; x_{\text {toe }}=83.88 ; h_{c}=0.32 ; x_{c}=85.25 ;$

$h_{\mathrm{t}}=0.70 ; \mathrm{X}_{\mathrm{t}}=85.65 ; \beta_{1}=15 ; \beta_{3}=40$.

$\mathrm{H}$

$\mathrm{X}$

$\mathrm{h}$

$\eta$

$\begin{array}{llll}0.46 & 30.00 & 1.25 & -0.007 \\ 0.61 & 80.92 & 0.85 & -0.009 \\ 0.71 & 83.50 & 0.78 & 0.026 \\ 0.57 & 84.50 & 0.51 & -0.018 \\ 0.23 & 86.75 & 0.65 & -0.001 \\ 0.26 & 89.75 & 0.61 & 0.011 \\ 0.24 & 93.00 & 0.46 & 0.023 \\ 0.13 & 98.00 & 0.31 & 0.033\end{array}$


Case 6440: $T=1.50 ; \mathrm{H}_{\mathrm{b}}=0.00 ; \mathrm{X}_{\mathrm{b}}=0.00 ; \mathrm{h}_{\mathrm{b}}=0.00 ; \mathrm{H}_{\mathrm{o}} / \mathrm{L}_{\mathrm{o}}=0.050 ;$

$\mathrm{h}_{\mathrm{tOe}}=0.76 ; \mathrm{x}_{\mathrm{tOe}}=84.17 ; \mathrm{h}_{\mathrm{c}}=0.33 ; \mathrm{x}_{\mathrm{c}}=85.17 ;$

$\mathrm{h}_{\mathrm{t}}=0.70 ; \mathrm{X}_{\mathrm{t}}=85.58 ; \beta_{1}=20 ; \beta_{3}=40$.

$\mathrm{H} \quad \mathrm{X}$ h

$\begin{array}{lllr}0.46 & 30.00 & 1.25 & -0.007 \\ 0.62 & 80.92 & 0.85 & -0.008 \\ 0.64 & 83.50 & 0.78 & 0.032 \\ 0.55 & 84.58 & 0.51 & -0.022 \\ 0.29 & 86.75 & 0.65 & -0.001 \\ 0.28 & 88.75 & 0.61 & 0.008 \\ 0.24 & 93.00 & 0.46 & 0.022 \\ 0.11 & 98.00 & 0.31 & 0.033\end{array}$

Case 8110: $T=1.74 ; \mathrm{H}_{b}=0.55 ; \mathrm{X}_{\mathrm{b}}=82.78 ; \mathrm{h}_{\mathrm{b}}=0.53 ; \mathrm{H}_{\mathrm{o}} / \mathrm{L}_{\mathrm{o}}=0.031 ;$

$\mathrm{h}_{\text {toe }}=0.90 ; \mathrm{x}_{\text {toe }}=79.83 ; \mathrm{h}_{\mathrm{c}}=0.31 ; \mathrm{x}_{\mathrm{c}}=85.38$;

$\mathrm{h}_{\mathrm{t}}=0.34 ; \mathrm{x}_{\mathrm{t}}=94.90 ; \beta_{1}=5 ; \beta_{3}=0$.

$\mathrm{H}$

$\mathrm{X}$

h

$\eta$

$\begin{array}{lrrr}0.46 & 30.00 & 1.26 & -0.014 \\ 0.47 & 75.90 & 1.03 & -0.015 \\ 0.51 & 79.50 & 0.92 & -0.015 \\ 0.53 & 83.15 & 0.51 & -0.026 \\ 0.18 & 87.35 & 0.31 & 0.039 \\ 0.16 & 91.00 & 0.36 & 0.051 \\ 0.12 & 95.00 & 0.36 & 0.053 \\ 0.12 & 100.00 & 0.25 & 0.055\end{array}$

Case 8120: $T=1.74 ; H_{b}=0.57 ; X_{b}=84.46 ; h_{b}=0.45 ; H_{o} / L_{\circ}=0.031 ;$

$\mathrm{h}_{\text {toe }}=0.79 ; \mathrm{X}_{\text {toe }}=82.95 ; \mathrm{h}_{\mathrm{c}}=0.30 ; \mathrm{X}_{\mathrm{c}}=85.35 ;$

$h_{\mathrm{t}}=0.33 ; \mathrm{X}_{\mathrm{t}}=94.90 ; \beta_{1}=10 ; \beta_{3}=0$.

$\mathrm{H}$

$\mathrm{X}$

$\mathrm{h}$

$\eta$

0.47

30.00

1.26

$-0.015$

0.50

79.50

0.91

$-0.014$

0.53

82.50

0.81

$-0.016$

0.54

83.88

0.55

$-0.021$

0.29

87.00

0.31

0.034

0.17

91.00

0.35

0.061

0.12

95.00

0.35

0.064

0.13

100.00

0.23

0.063 
Case 8130: $T=1.74 ; \mathrm{H}_{\mathrm{b}}=0.66 ; \mathrm{X}_{\mathrm{b}}=84.45 ; \mathrm{h}_{\mathrm{b}}=0.56 ; \mathrm{H}_{\mathrm{o}} / \mathrm{L}_{\mathrm{o}}=0.032 ;$

$h_{\text {toe }}=0.76 ; x_{\text {toe }}=83.90 ; h_{c}=0.32 ; x_{c}=85.38 ;$

$h_{\mathrm{t}}=0.34 ; \mathrm{x}_{\mathrm{t}}=94.90 ; \beta_{1}=15 ; \beta_{3}=0$.

$\mathrm{H} \quad \mathrm{X} \quad \mathrm{h} \quad \eta$

$\begin{array}{lrrr}0.48 & 30.00 & 1.26 & -0.014 \\ 0.53 & 80.75 & 0.86 & -0.014 \\ 0.75 & 83.25 & 0.79 & -0.007 \\ 0.60 & 84.45 & 0.55 & -0.018 \\ 0.15 & 87.25 & 0.31 & 0.021 \\ 0.20 & 91.00 & 0.35 & 0.058 \\ 0.12 & 95.00 & 0.35 & 0.064 \\ 0.13 & 100.00 & 0.23 & 0.063\end{array}$

Case 8140: $T=1.74 ; H_{b}=0.66 ; X_{b}=84.42 ; h_{b}=0.66 ; H_{o} / L_{o}=0.032 ;$

$h_{\text {toe }}=0.75 ; X_{\text {toe }}=84.30 ; h_{c}=0.32 ; X_{c}=85.35 ;$

$h_{t}=0.33 ; X_{t}=94.90 ; \beta_{1}=20 ; \beta_{3}=0$.

$\begin{array}{lllll}\mathrm{H} & \mathrm{X} & \mathrm{h} & \eta\end{array}$

$\begin{array}{lrrr}0.48 & 30.00 & 1.26 & -0.012 \\ 0.52 & 80.70 & 0.86 & -0.010 \\ 0.53 & 83.70 & 0.77 & -0.017 \\ 0.57 & 84.38 & 0.52 & -0.024 \\ 0.17 & 87.25 & 0.31 & 0.019 \\ 0.20 & 91.00 & 0.35 & 0.057 \\ 0.11 & 95.00 & 0.34 & 0.063 \\ 0.13 & 100.00 & 0.23 & 0.065\end{array}$

Case 8210: $T=1.74 ; H_{b}=0.48 ; X_{b}=82.83 ; h_{b}=0.56 ; H_{o} / L_{o}=0.032 ;$

$h_{\text {toe }}=0.90 ; x_{\text {toe }}=79.85 ; h_{c}=0.36 ; x_{c}=85.13$;

$h_{\mathrm{t}}=0.69 ; \mathrm{X}_{\mathrm{t}}=85.83 ; \beta_{1}=5 ; \beta_{3}=20$.

$\begin{array}{cccc}\text { H } & \mathrm{X} & \mathrm{h} & \eta \\ 0.48 & 30.00 & 1.25 & -0.004 \\ 0.46 & 76.40 & 1.01 & -0.008 \\ 0.66 & 79.40 & 0.92 & 0.009 \\ 0.52 & 83.70 & 0.49 & -0.013 \\ 0.19 & 86.70 & 0.66 & 0.008 \\ 0.28 & 90.50 & 0.56 & 0.021 \\ 0.26 & 94.50 & 0.44 & 0.022 \\ 0.17 & 100.00 & 0.24 & 0.029\end{array}$


Case 8220: $T=1.74 ; \mathrm{H}_{\mathrm{b}}=0.59 ; \mathrm{X}_{\mathrm{b}}=85.18 ; \mathrm{h}_{\mathrm{b}}=0.41 ; \mathrm{H}_{\mathrm{o}} / \mathrm{L}_{\mathrm{o}}=0.034 ;$

$h_{\text {toe }}=0.78 ; x_{\text {toe }}=83.60 ; h_{c}=0.34 ; x_{c}=85.60 ;$

$h_{\mathrm{t}}=0.67 ; \mathrm{X}_{\mathrm{t}}=86.31 ; \beta_{1}=10 ; \beta_{3}=20$.

$\mathrm{H} \quad \mathrm{X} \quad \mathrm{h} \quad \boldsymbol{\eta}$

$\begin{array}{lrrr}0.50 & 30.00 & 1.25 & -0.004 \\ 0.57 & 80.42 & 0.87 & -0.003 \\ 0.70 & 83.42 & 0.78 & 0.017 \\ 0.62 & 84.75 & 0.49 & -0.015 \\ 0.25 & 87.67 & 0.63 & -0.001 \\ 0.27 & 90.50 & 0.56 & 0.016 \\ 0.21 & 94.50 & 0.43 & 0.022 \\ 0.13 & 100.00 & 0.24 & 0.035\end{array}$

Case 8230: $T=1.74 ; \mathrm{H}_{\mathrm{b}}=0.54 ; \mathrm{X}_{\mathrm{b}}=85.15 ; \mathrm{h}_{\mathrm{b}}=0.50 ; \mathrm{H}_{\mathrm{o}} / \mathrm{L}_{\mathrm{o}}=0.034 ;$

$h_{\text {toe }}=0.75 ; x_{\text {toe }}=84.40 ; h_{c}=0.36 ; x_{c}=85.65$;

$h_{\mathrm{t}}=0.67 ; \mathrm{X}_{\mathrm{t}}=86.35 ; \beta_{1}=15 ; \beta_{3}=20$.

$\mathrm{H}$

$\mathrm{X}$

h

$\eta$

0.50

30.00

1.25

$-0.004$

0.54

0.82

$-0.006$

0.74

0.75

0.030

0.58

0.50

$-0.017$

0.31

0.63

0.004

0.30

0.55

0.018

0.22

0.43

0.023

0.12

94.50

0.24

0.039

Case 8240: $\quad T=1.74 ; H_{b}=0.58 ; X_{b}=84.71 ; h_{b}=0.61 ; H_{o} / L_{o}=0.034 ;$

$h_{\text {toe }}=0.74 ; x_{\text {toe }}=84.71 ; h_{c}=0.32 ; x_{c}=85.50 ;$

$h_{\mathrm{t}}=0.67 ; \mathrm{X}_{\mathrm{t}}=86.33 ; \beta_{1}=20 ; \beta_{3}=20$.

$\mathrm{H}$ $\mathrm{X}$

h

0.51

30.00

1.25

$-0.006$

0.60

80.75

0.86

$-0.008$

0.70

83.75

0.76

0.022

0.60

85.00

0.56

$-0.011$

0.29

87.50

0.63

0.005

0.30

90.50

0.54

0.014

0.23

94.50

0.43

0.027

0.14

100.00

0.23

0.040 
Case 8310: $T=1.74 ; \mathrm{H}_{\mathrm{b}}=0.50 ; \mathrm{X}_{\mathrm{b}}=82.80 ; \mathrm{h}_{\mathrm{b}}=0.58 ; \mathrm{H}_{\mathrm{o}} / \mathrm{L}_{\mathrm{o}}=0.032 ;$

$\mathrm{h}_{\text {toe }}=0.89 ; \mathrm{x}_{\text {toe }}=79.92 ; \mathrm{h}_{\mathrm{c}}=0.38 ; \mathrm{x}_{\mathrm{c}}=85.05 ;$

$\mathrm{h}_{\mathrm{t}}=0.71 ; \mathrm{x}_{\mathrm{t}}=85.52 ; \beta_{1}=5 ; \beta_{3}=30$.

\begin{tabular}{lrrr} 
H & X & \multicolumn{1}{c}{ h } & $\eta$ \\
0.48 & 30.00 & 1.25 & -0.007 \\
0.46 & 76.25 & 1.02 & 0.000 \\
0.58 & 79.75 & 0.90 & -0.004 \\
0.54 & 83.60 & 0.55 & -0.011 \\
0.23 & 87.00 & 0.65 & 0.007 \\
0.28 & 91.00 & 0.52 & 0.017 \\
0.26 & 95.00 & 0.41 & 0.019 \\
0.16 & 100.00 & 0.23 & 0.031
\end{tabular}

Case 8320: $T=1.74 ; \mathrm{H}_{\mathrm{b}}=0.60 ; \mathrm{X}_{\mathrm{b}}=84.41 ; \mathrm{h}_{\mathrm{b}}=0.53 ; \mathrm{H}_{\mathrm{o}} / \mathrm{L}_{\mathrm{o}}=0.032 ;$

$\mathrm{h}_{\text {toe }}=0.78 ; \mathrm{x}_{\text {toe }}=83.50 ; \mathrm{h}_{\mathrm{c}}=0.35 ; \mathrm{x}_{\mathrm{c}}=85.44 ;$

$\mathrm{h}_{\mathrm{t} .}=0.69 ; \mathrm{x}_{\mathrm{t}}=85.90 ; \beta_{1}=10 ; \beta_{3}=30$.

$\mathrm{H}$ $\mathrm{X}$

h

$\eta$

0.50

30.00

1.25

$-0.005$

0.58

80.25

0.88

$-0.001$

0.72

83.25

0.79

0.014

0.61

84.45

0.54

$-0.012$

0.29

87.50

0.64

0.004

0.27

90.50

0.55

0.009

0.23

94.50

0.43

0.022

0.13

100.00

0.24

0.035

Case 8330: $T=1.74 ; \mathrm{H}_{\mathrm{b}}=0.56 ; \mathrm{X}_{\mathrm{b}}=85.26 ; \mathrm{h}_{\mathrm{b}}=0.48 ; \mathrm{H}_{\mathrm{o}} / \mathrm{L}_{\mathrm{o}}=0.034 ;$

$\mathrm{h}_{\text {toe }}=0.74 ; \mathrm{x}_{\text {toe }}=84.60 ; \mathrm{h}_{\mathrm{c}}=0.41 ; \mathrm{x}_{\mathrm{c}}=85.75$;

$h_{\mathrm{t}}=0.67 ; \mathrm{x}_{\mathrm{t}}=86.25 ; \beta_{1}=15 ; \beta_{3}=30$.

$\begin{array}{cccc}\mathrm{H} & \mathrm{X} & \mathrm{h} & \eta \\ 0.50 & 30.00 & 1.25 & -0.006 \\ 0.58 & 81.00 & 0.85 & -0.003 \\ 0.79 & 84.00 & 0.75 & 0.014 \\ 0.60 & 85.08 & 0.61 & -0.011 \\ 0.31 & 87.50 & 0.63 & -0.006 \\ 0.30 & 90.50 & 0.55 & 0.010 \\ 0.21 & 94.50 & 0.43 & 0.021 \\ 0.12 & 100.00 & 0.24 & 0.035\end{array}$


Case 8340: $T=1.74 ; \mathrm{H}_{\mathrm{b}}=0.56 ; \mathrm{X}_{\mathrm{b}}=84.80 ; \mathrm{h}_{\mathrm{b}}=0.69 ; \mathrm{H}_{\mathrm{o}} / \mathrm{L}_{\mathrm{o}}=0.034 ;$

$h_{\text {toe }}=0.74 ; x_{\text {toe }}=84.80 ; h_{c}=0.34 ; x_{c}=85.67$;

$h_{\mathrm{t}}=0.67 ; \mathrm{X}_{\mathrm{t}}=86.15 ; \beta_{1}=20 ; \beta_{3}=30$.

$\begin{array}{lrrr}\mathrm{H} & \mathrm{X} & \mathrm{h} & \eta \\ 0.50 & 30.00 & 1.25 & -0.005 \\ 0.58 & 80.90 & 0.85 & -0.007 \\ 0.74 & 83.95 & 0.76 & 0.015 \\ 0.57 & 85.10 & 0.55 & -0.009 \\ 0.30 & 87.50 & 0.63 & -0.001 \\ 0.27 & 90.50 & 0.55 & 0.007 \\ 0.22 & 94.50 & 0.43 & 0.023 \\ 0.12 & 100.00 & 0.24 & 0.039\end{array}$

Case 8410: $T=1.74 ; \mathrm{H}_{\mathrm{b}}=0.52 ; \mathrm{X}_{\mathrm{b}}=83.27 ; \mathrm{h}_{\mathrm{b}}=0.54 ; \mathrm{H}_{\mathrm{o}} / \mathrm{L}_{\mathrm{o}}=0.033 ;$

$\mathrm{h}_{\text {toe }}=0.88 ; \mathrm{x}_{\text {toe }}=80.29 ; \mathrm{h}_{\mathrm{c}}=0.36 ; \mathrm{x}_{\mathrm{c}}=85.33 ;$

$h_{t}=0.70 ; X_{t}=85.65 ; \beta_{1}=5 ; \beta_{3}=40$.

$\begin{array}{lccc}\text { H } & \text { X } & \text { h } & \eta \\ 0.48 & 30.00 & 1.25 & -0.005 \\ 0.47 & 76.50 & 1.01 & -0.007 \\ 0.56 & 80.00 & 0.88 & -0.003 \\ 0.59 & 83.45 & 0.53 & -0.014 \\ 0.20 & 87.00 & 0.66 & 0.009 \\ 0.28 & 91.00 & 0.53 & 0.020 \\ 0.25 & 95.00 & 0.41 & 0.023 \\ 0.15 & 100.00 & 0.23 & 0.031\end{array}$

Case 8420: $T=1.74 ; H_{b}=0.54 ; X_{b}=84.53 ; h_{b}=0.50 ; H_{o} / L_{o}=0.034 ;$

$\mathrm{h}_{\text {toe }}=0.78 ; \mathrm{x}_{\text {toe }}=83.50 ; \mathrm{h}_{\mathrm{c}}=0.35 ; \mathrm{x}_{\mathrm{c}}=85.44 ;$

$h_{t}=0.69 ; X_{t}=85.77 ; \beta_{1}=10 ; \beta_{3}=40$.

$\begin{array}{cccc}\text { H } & \mathrm{X} & \mathrm{h} & \eta \\ 0.50 & 30.00 & 1.25 & -0.006 \\ 0.57 & 80.00 & 0.88 & -0.005 \\ 0.82 & 83.00 & 0.79 & 0.000 \\ 0.61 & 84.13 & 0.58 & -0.009 \\ 0.22 & 87.00 & 0.65 & -0.014 \\ 0.30 & 91.00 & 0.52 & 0.013 \\ 0.24 & 95.00 & 0.41 & 0.023 \\ 0.14 & 100.00 & 0.23 & 0.038\end{array}$


Case 8430: $T=1.74 ; \mathrm{H}_{\mathrm{b}}=0.54 ; \mathrm{X}_{\mathrm{b}}=84.85 ; \mathrm{h}_{\mathrm{b}}=0.49 ; \mathrm{H}_{\mathrm{o}} / \mathrm{L}_{\mathrm{o}}=0.034 ;$

$\mathrm{h}_{\text {toe }}=0.76 ; \mathrm{x}_{\text {toe }}=84.08 ; \mathrm{h}_{\mathrm{c}}=0.38 ; \mathrm{X}_{\mathrm{c}}=85.25 ;$

$h_{t}=0.70 ; X_{t}=85.60 ; \beta_{1}=15 ; \beta_{3}=40$.

$\begin{array}{cccc}\mathrm{H} & \mathrm{X} & \mathrm{h} & \eta \\ 0.50 & 30.00 & 1.25 & -0.005 \\ 0.56 & 80.75 & 0.86 & -0.005 \\ 0.80 & 83.25 & 0.79 & 0.009 \\ 0.62 & 84.44 & 0.59 & -0.008 \\ 0.29 & 87.00 & 0.66 & -0.008 \\ 0.29 & 91.00 & 0.54 & 0.008 \\ 0.22 & 95.00 & 0.41 & 0.021 \\ 0.13 & 100.00 & 0.24 & 0.037\end{array}$

Case 8440: $T=1.74 ; H_{b}=0.54 ; X_{b}=84.84 ; h_{b}=0.65 ; H_{o} / L_{o}=0.034 ;$

$h_{\text {toe }}=0.74 ; x_{\text {toe }}=84.75 ; h_{c}=0.37 ; x_{c}=85.60 ;$

$\mathrm{h}_{\mathrm{t}}=0.69 ; \mathrm{x}_{\mathrm{t}}=85.95 ; \beta_{1}=20 ; \beta_{3}=40$.

$\mathrm{H}$

$\mathrm{X}$

h

$\eta$

$\begin{array}{lrrr}0.50 & 30.00 & 1.25 & -0.003 \\ 0.56 & 81.25 & 0.85 & 0.001 \\ 0.82 & 83.75 & 0.77 & 0.008 \\ 0.61 & 84.85 & 0.64 & -0.009 \\ 0.30 & 87.00 & 0.65 & -0.009 \\ 0.29 & 91.00 & 0.53 & 0.009 \\ 0.22 & 95.00 & 0.41 & 0.021 \\ 0.12 & 100.00 & 0.24 & 0.037\end{array}$

Case 10110: $\mathrm{T}=2.49 ; \mathrm{H}_{\mathrm{b}}=0.44 ; \mathrm{X}_{\mathrm{b}}=89.83 ; \mathrm{h}_{\mathrm{b}}=0.37 ; \mathrm{H}_{\mathrm{o}} / \mathrm{L}_{\mathrm{o}}=0.010 ;$

$h_{\text {toe }}=0.64 ; x_{\text {toe }}=87.55 ; h_{c}=0.25 ; X_{c}=91.25 ;$

$h_{\mathrm{t}}=0.26 ; \mathrm{x}_{\mathrm{t}}=97.80 ; \beta_{1}=5 ; \beta_{3}=0$.

$\mathrm{H}$

$\mathrm{X}$

$\mathrm{h}$

$\eta$

$\begin{array}{lrrr}0.34 & 30.00 & 1.26 & -0.007 \\ 0.33 & 83.50 & 0.78 & -0.007 \\ 0.35 & 87.50 & 0.63 & -0.006 \\ 0.44 & 89.90 & 0.38 & -0.016 \\ 0.17 & 92.50 & 0.24 & 0.020 \\ 0.14 & 94.50 & 0.24 & 0.037 \\ 0.11 & 97.00 & 0.25 & 0.048 \\ 0.13 & 100.00 & 0.23 & 0.050\end{array}$


Case 10120: $T=2.48 ; H_{b}=0.44 ; X_{b}=90.26 ; h_{b}=0.42 ; H_{o} / L_{o}=0.008 ;$

$\mathrm{h}_{\text {toe }}=0.58 ; \mathrm{x}_{\text {toe }}=89.85 ; \mathrm{h}_{\mathrm{c}}=0.25 ; \mathrm{x}_{\mathrm{c}}=91.25 ;$

$h_{\mathrm{t}}=0.26 ; \mathrm{X}_{\mathrm{t}}=97.80 ; \beta_{1}=10 ; \beta_{3}=0$.

$\begin{array}{lrcc}\text { H } & \text { X } & \text { h } & \eta \\ 0.27 & 30.00 & 1.26 & -0.010 \\ 0.31 & 85.50 & 0.71 & -0.008 \\ 0.34 & 89.50 & 0.59 & -0.010 \\ 0.39 & 90.50 & 0.40 & -0.013 \\ 0.20 & 92.50 & 0.24 & 0.017 \\ 0.13 & 94.50 & 0.24 & 0.042 \\ 0.12 & 97.00 & 0.25 & 0.044 \\ 0.08 & 100.00 & 0.23 & 0.051\end{array}$

Case 10130: $T=2.49 ; H_{b}=0.40 ; x_{b}=90.29 ; h_{b}=0.50 ; H_{o} / L_{o}=0.008 ;$

$\mathrm{h}_{\text {toe }}=0.56 ; \mathrm{x}_{\text {toe }}=90.40 ; \mathrm{h}_{\mathrm{c}}=0.24 ; \mathrm{x}_{\mathrm{c}}=91.25$;

$h_{\mathrm{t}}=0.26 ; \mathrm{X}_{\mathrm{t}}=97.80 ; \beta_{1}=15 ; \beta_{3}=0$.

$\begin{array}{lllll}\mathrm{H} & \mathrm{X} & \mathrm{h} & \eta\end{array}$

$\begin{array}{lrrr}0.27 & 30.00 & 1.26 & -0.009 \\ 0.32 & 85.50 & 0.71 & -0.008 \\ 0.32 & 89.50 & 0.58 & -0.012 \\ 0.39 & 90.75 & 0.39 & -0.016 \\ 0.15 & 92.50 & 0.24 & 0.011 \\ 0.14 & 94.50 & 0.23 & 0.038 \\ 0.12 & 97.00 & 0.25 & 0.042 \\ 0.11 & 100.00 & 0.24 & 0.049\end{array}$

Case 10210: $T=2.49 ; H_{b}=0.39 ; X_{b}=89.65 ; h_{b}=0.38 ; H_{o} / L_{o}=0.009 ;$

$\mathrm{h}_{\text {toe }}=0.64 ; \mathrm{x}_{\text {toe }}=87.63 ; \mathrm{h}_{\mathrm{c}}=0.26 ; \mathrm{x}_{\mathrm{c}}=91.05 ;$

$h_{\mathrm{t}}=0.50 ; \mathrm{x}_{\mathrm{t}}=91.54 ; \beta_{1}=5 ; \beta_{3}=20$.

$\begin{array}{cccc}\mathrm{H} & \mathrm{X} & \mathrm{h} & \eta \\ 0.30 & 30.00 & 1.26 & -0.005 \\ 0.31 & 83.50 & 0.78 & -0.004 \\ 0.32 & 87.50 & 0.64 & -0.008 \\ 0.40 & 89.48 & 0.41 & -0.010 \\ 0.17 & 91.75 & 0.50 & 0.000 \\ 0.17 & 94.00 & 0.45 & 0.012 \\ 0.19 & 97.00 & 0.34 & 0.015 \\ 0.14 & 100.00 & 0.24 & 0.022\end{array}$


Case 10220: $T=2.49 ; H_{b}=0.37 ; X_{b}=89.62 ; h_{b}=0.39 ; H_{o} / L_{o}=0.009 ;$

$h_{\text {toe }}=0.60 ; x_{\text {toe }}=89.05 ; h_{c}=0.26 ; x_{c}=90.35 ;$

$h_{\mathrm{t}}=0.53 ; \mathrm{X}_{\mathrm{t}}=90.80 ; \beta_{1}=10 ; \beta_{3}=20$.

$\begin{array}{lrcc}\mathrm{H} & \mathrm{X} & \mathrm{h} & \eta \\ 0.30 & 30.00 & 1.26 & -0.004 \\ 0.33 & 84.50 & 0.74 & -0.005 \\ 0.37 & 88.50 & 0.61 & -0.006 \\ 0.42 & 89.60 & 0.40 & -0.011 \\ 0.18 & 92.00 & 0.49 & 0.000 \\ 0.13 & 94.00 & 0.44 & 0.009 \\ 0.18 & 97.00 & 0.33 & 0.016 \\ 0.10 & 100.00 & 0.23 & 0.026\end{array}$

Case 10230: $T=2.49 ; \mathrm{H}_{\mathrm{b}}=0.41 ; \mathrm{X}_{\mathrm{b}}=89.73 ; \mathrm{h}_{\mathrm{b}}=0.56 ; \mathrm{H}_{\mathrm{o}} / \mathrm{L}_{\mathrm{o}}=0.008 ;$

$h_{\text {toe }}=0.58 ; x_{\text {toe }}=89.73 ; h_{c}=0.27 ; x_{c}=90.53 ;$

$h_{\mathrm{t}}=0.53 ; \mathrm{x}_{\mathrm{t}}=91.00 ; \beta_{1}=15 ; \beta_{3}=20$.

$\mathrm{H} \quad \mathrm{X} \quad \mathrm{h} \quad \eta$

$\begin{array}{lrrr}0.27 & 30.00 & 1.26 & -0.005 \\ 0.33 & 84.75 & 0.74 & -0.005 \\ 0.43 & 88.75 & 0.61 & -0.003 \\ 0.39 & 89.98 & 0.43 & -0.009 \\ 0.21 & 92.00 & 0.49 & 0.000 \\ 0.20 & 94.00 & 0.44 & 0.005 \\ 0.16 & 97.00 & 0.34 & 0.015 \\ 0.10 & 100.00 & 0.23 & 0.025\end{array}$

Case 10310: $T=2.49 ; \mathrm{H}_{\mathrm{b}}=0.41 ; \mathrm{x}_{\mathrm{b}}=90.55 ; \mathrm{h}_{\mathrm{b}}=0.36 ; \mathrm{H}_{\mathrm{o}} / \mathrm{L}_{\mathrm{o}}=0.008 ;$

$h_{\text {toe }}=0.63 ; x_{\text {toe }}=88.17 ; h_{c}=0.27 ; x_{c}=91.55 ;$

$h_{\mathrm{t}}=0.49 ; \mathrm{X}_{\mathrm{t}}=91.85 ; \beta_{1}=5 ; \beta_{3}=30$.

$\mathrm{H}$

$\mathrm{X}$

$\mathrm{h}$

$\eta$

0.28

30.00

1.26

$-0.005$

0.30

84:00

0.76

$-0.004$

0.39

88.00

0.63

$-0.006$

0.41

90.15

0.39

$-0.012$

0.18

93.00

0.46

0.002

0.20

95.00

0.41

0.008

0.19

97.00

0.34

0.015

0.16

100.00

0.23

0.021 
Case 10320: $T=2.49 ; \mathrm{H}_{\mathrm{b}}=0.36 ; \mathrm{x}_{\mathrm{b}}=90.18 ; \mathrm{h}_{\mathrm{b}}=0.38 ; \mathrm{H}_{\mathrm{o}} / \mathrm{L}_{\mathrm{o}}=0.008 ;$

$\mathrm{h}_{\text {toe }}=0.59 ; \mathrm{x}_{\text {toe }}=89.55 ; \mathrm{h}_{\mathrm{c}}=0.27 ; \mathrm{x}_{\mathrm{c}}=90.83$;

$h_{\mathrm{t}}=0.52 ; \mathrm{x}_{\mathrm{t}}=91.10 ; \beta_{1}=10 ; \beta_{3}=30$.

$\begin{array}{cccc}\mathrm{H} & \mathrm{X} & \mathrm{h} & \eta \\ 0.28 & 30.00 & 1.26 & -0.009 \\ 0.32 & 85.00 & 0.73 & -0.006 \\ 0.36 & 89.00 & 0.60 & -0.008 \\ 0.42 & 90.25 & 0.38 & -0.015 \\ 0.23 & 92.00 & 0.49 & -0.002 \\ 0.17 & 95.00 & 0.41 & 0.005 \\ 0.17 & 97.00 & 0.33 & 0.014 \\ 0.10 & 100.00 & 0.23 & 0.021\end{array}$

Case 10330: $T=2.49 ; \mathrm{H}_{\mathrm{b}}=0.40 ; \mathrm{X}_{\mathrm{b}}=90.30 ; \mathrm{h}_{\mathrm{b}}=0.54 ; \mathrm{H}_{\mathrm{o}} / \mathrm{L}_{\mathrm{o}}=0.008 ;$

$h_{\text {toe }}=0.56 ; x_{\text {toe }}=90.30 ; h_{c}=0.27 ; x_{c}=91.05 ;$

$h_{\mathrm{t}}=0.51 ; \mathrm{X}_{\mathrm{t}}=91.40 ; \beta_{1}=15 ; \beta_{3}=30$.

$\begin{array}{cccc}\text { H } & \text { X } & \text { h } & \eta \\ 0.27 & 30.00 & 1.26 & -0.008 \\ 0.32 & 85.25 & 0.72 & -0.007 \\ 0.34 & 89.25 & 0.60 & -0.007 \\ 0.41 & 90.45 & 0.44 & -0.011 \\ 0.21 & 92.00 & 0.49 & -0.001 \\ 0.22 & 95.00 & 0.41 & 0.003 \\ 0.17 & 97.00 & 0.33 & 0.013 \\ 0.09 & 100.00 & 0.23 & 0.021\end{array}$

Case 10410: $T=2.49 ; \mathrm{H}_{\mathrm{b}}=0.42 ; \mathrm{X}_{\mathrm{b}}=90.66 ; \mathrm{h}_{\mathrm{b}}=0.38 ; \mathrm{H}_{\mathrm{o}} / \mathrm{L}_{\mathrm{o}}=0.008 ;$

$\mathrm{h}_{\text {toe }}=0.61 ; \mathrm{x}_{\text {toe }}=88.60 ; \mathrm{h}_{\mathrm{c}}=0.27 ; \mathrm{x}_{\mathrm{c}}=91.90 ;$

$h_{\mathrm{t}}=0.49 ; \mathrm{x}_{\mathrm{t}}=92.10 ; \beta_{1}=5 ; \beta_{3}=40$.

$\begin{array}{llll}\mathrm{H} & \mathrm{X} & \mathrm{h}\end{array}$

$\begin{array}{lrrr}0.27 & 30.00 & 1.26 & -0.007 \\ 0.31 & 84.50 & 0.75 & -0.006 \\ 0.34 & 88.50 & 0.61 & -0.008 \\ 0.42 & 90.40 & 0.39 & -0.012 \\ 0.15 & 93.10 & 0.46 & -0.002 \\ 0.19 & 95.00 & 0.41 & 0.007 \\ 0.20 & 97.00 & 0.34 & 0.015 \\ 0.12 & 100.00 & 0.24 & 0.020\end{array}$


Case 10420: $T=2.49 ; H_{b}=0.37 ; X_{b}=90.64 ; h_{b}=0.40 ; H_{o} / L_{o}=0.008 ;$

$\mathrm{h}_{\text {toe }}=0.57 ; \mathrm{x}_{\text {toe }}=90.13 ; \mathrm{h}_{\mathrm{c}}=0.26 ; \mathrm{x}_{\mathrm{c}}=91.40 ;$

$h_{\mathrm{t}}=0.50 ; \mathrm{X}_{\mathrm{t}}=91.63 ; \beta_{1}=10 ; \beta_{3}=40$.

$\mathrm{H} \quad \mathrm{X} \quad \mathrm{h} \quad \eta$

$\begin{array}{lrrr}0.26 & 30.00 & 1.26 & -0.004 \\ 0.31 & 85.50 & 0.71 & -0.006 \\ 0.37 & 89.50 & 0.59 & -0.007 \\ 0.42 & 90.70 & 0.38 & -0.010 \\ 0.20 & 92.50 & 0.47 & -0.001 \\ 0.19 & 94.50 & 0.44 & 0.005 \\ 0.19 & 97.00 & 0.34 & 0.012 \\ 0.10 & 100.00 & 0.24 & 0.022\end{array}$

Case 10430: $T=2.49 ; H_{b}=0.38 ; X_{b}=90.63 ; h_{b}=0.52 ; H_{o} / L_{o}=0.008 ;$

$h_{\text {toe }}=0.55 ; x_{\text {toe }}=90.63 ; h_{c}=0.26 ; x_{c}=91.42 ;$

$h_{\mathrm{t}}=0.50 ; \mathrm{x}_{\mathrm{t}}=91.65 ; \beta_{1}=15 ; \beta_{3}=40$.

$\mathrm{H} \quad \mathrm{X} \quad \mathrm{h} \quad \eta$

$\begin{array}{lrrr}0.27 & 30.00 & 1.26 & -0.006 \\ 0.32 & 85.50 & 0.71 & -0.007 \\ 0.31 & 89.50 & 0.58 & -0.007 \\ 0.43 & 90.90 & 0.39 & -0.008 \\ 0.17 & 92.50 & 0.47 & 0.000 \\ 0.19 & 94.50 & 0.44 & 0.003 \\ 0.18 & 97.00 & 0.34 & 0.013 \\ 0.11 & 100.00 & 0.24 & 0.023\end{array}$

PLANE SLOPE MONOCHROMATIC TESTS

Case 2000: $T=1.02 ; H_{b}=0.40 ; X_{b}=86.53 ; h_{b}=0.67 ; H_{o} / L_{o}=0.092 ;$

$\mathrm{m}=0.033$

$\begin{array}{cccc}\mathrm{H} & \mathrm{X} & \mathrm{h} & \eta \\ 0.45 & 30.00 & 1.26 & 0.000 \\ 0.42 & 83.00 & 0.80 & -0.004 \\ 0.43 & 84.50 & 0.75 & -0.004 \\ 0.44 & 86.65 & 0.66 & -0.006 \\ 0.27 & 90.00 & 0.57 & -0.008 \\ 0.19 & 93.00 & 0.46 & 0.006 \\ 0.15 & 96.00 & 0.38 & 0.021 \\ 0.15 & 100.00 & 0.24 & 0.027\end{array}$


Case 4000: $T=1.02 ; H_{b}=0.27 ; X_{b}=93.70 ; h_{b}=0.43 ; H_{o} / L_{o}=0.066 ;$ $\mathrm{m}=0.033$

$\begin{array}{cccc}\mathrm{H} & \mathrm{X} & \mathrm{h} & \eta \\ 0.32 & 30.00 & 1.26 & -0.002 \\ 0.30 & 91.00 & 0.53 & -0.002 \\ 0.30 & 92.25 & 0.48 & -0.007 \\ 0.30 & 93.96 & 0.44 & -0.004 \\ 0.21 & 95.05 & 0.41 & -0.004 \\ 0.19 & 96.50 & 0.36 & -0.003 \\ 0.14 & 98.25 & 0.30 & 0.010 \\ 0.12 & 100.00 & 0.23 & 0.016\end{array}$

Case 6000: $T=1.49 ; H_{b}=0.51 ; X_{b}=85.32 ; h_{b}=0.71 ; H_{o} / L_{o}=0.046 ;$ $\mathrm{m}=0.033$

$\begin{array}{cccc}\text { H } & \mathrm{X} & \mathrm{h} & \eta \\ 0.48 & 30.00 & 1.26 & -0.003 \\ 0.54 & 81.50 & 0.84 & -0.009 \\ 0.54 & 84.00 & 0.76 & -0.011 \\ 0.49 & 85.95 & 0.68 & -0.015 \\ 0.32 & 89.00 & 0.60 & -0.011 \\ 0.28 & 91.00 & 0.52 & -0.007 \\ 0.19 & 95.00 & 0.41 & 0.021 \\ 0.15 & 100.00 & 0.24 & 0.039\end{array}$

Case 8000: $T=1.74 ; H_{b}=0.54 ; X_{b}=85.09 ; h_{b}=0.71 ; H_{o} / L_{o}=0.030 ;$ $\mathrm{m}=0.033$

$\begin{array}{cccc}\mathrm{H} & \mathrm{X} & \mathrm{h} & \eta \\ 0.45 & 30.00 & 1.26 & -0.008 \\ 0.50 & 80.75 & 0.86 & -0.008 \\ 0.56 & 84.00 & 0.76 & -0.007 \\ 0.49 & 85.90 & 0.69 & -0.007 \\ 0.31 & 89.00 & 0.60 & -0.005 \\ 0.32 & 91.00 & 0.53 & 0.004 \\ 0.19 & 95.00 & 0.41 & 0.021 \\ 0.15 & 100.00 & 0.24 & 0.042\end{array}$


Case 10000: $T=2.49 ; H_{b}=0.42 ; X_{b}=91.68 ; h_{b}=0.49 ; H_{o} / L_{o}=0.009 ;$ $\mathrm{m}=0.033$

$\begin{array}{lrrc}\text { H } & \text { X } & \text { h } & \eta \\ 0.30 & 30.00 & 1.26 & -0.003 \\ 0.34 & 87.70 & 0.63 & -0.006 \\ 0.44 & 91.70 & 0.50 & -0.006 \\ 0.35 & 93.55 & 0.45 & -0.004 \\ 0.22 & 94.95 & 0.41 & -0.003 \\ 0.16 & 96.50 & 0.35 & 0.003 \\ 0.17 & 98.25 & 0.30 & 0.014 \\ 0.08 & 100.00 & 0.24 & 0.023\end{array}$

IRREGULAR TESTS

KEY:

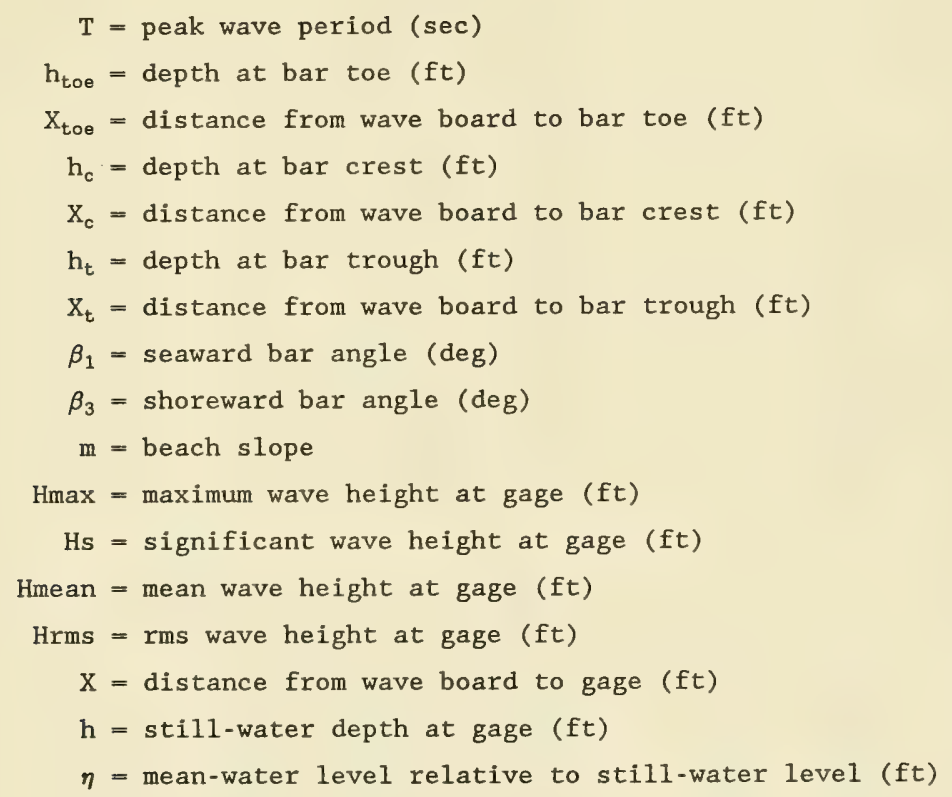


Case R2210: $T=1.00$

$h_{\text {toe }}=0.72 ; x_{\text {toe }}=85.40 ; h_{c}=0.24 ; X_{c}=89.85 ;$

$\mathrm{h}_{\mathrm{t}}=0.56 ; \mathrm{x}_{\mathrm{t}}=90.45 ; \beta_{1}=5 ; \beta_{3}=20$.

\begin{tabular}{lcccccc} 
Hmax & Hs & Hmean & Hrms & $\mathrm{X}$ & $\mathrm{h}$ & \multicolumn{1}{c}{$\eta$} \\
0.49 & 0.37 & 0.25 & 0.27 & 30.00 & 1.25 & -0.004 \\
0.43 & 0.31 & 0.21 & 0.23 & 84.50 & 0.75 & 0.000 \\
0.45 & 0.31 & 0.21 & 0.23 & 85.00 & 0.74 & -0.002 \\
0.38 & 0.30 & 0.21 & 0.23 & 88.00 & 0.40 & -0.004 \\
0.21 & 0.15 & 0.11 & 0.12 & 91.00 & 0.54 & 0.005 \\
0.24 & 0.18 & 0.13 & 0.14 & 93.00 & 0.42 & 0.007 \\
0.23 & 0.17 & 0.13 & 0.14 & 96.00 & 0.38 & 0.008 \\
0.26 & 0.18 & 0.14 & 0.15 & 100.00 & 0.24 & 0.011
\end{tabular}

Case R2220: $\quad T=1.00$

$\mathrm{h}_{\text {toe }}=0.61 ; \mathrm{x}_{\text {toe }}=88.80 ; \mathrm{h}_{\mathrm{c}}=0.23 ; \mathrm{x}_{\mathrm{c}}=90.50 ;$

$\mathrm{h}_{\mathrm{t}}=0.52 ; \mathrm{X}_{\mathrm{t}}=91.08 ; \beta_{1}=10 ; \beta_{3}=20$.

$\begin{array}{lcccccc}\text { Hmax } & \text { Hs } & \text { Hmean } & \text { Hrms } & \mathrm{X} & \mathrm{h} & \eta \\ 0.50 & 0.37 & 0.25 & 0.27 & 30.00 & 1.25 & -0.003 \\ 0.43 & 0.32 & 0.22 & 0.24 & 87.75 & 0.63 & 0.002 \\ 0.41 & 0.31 & 0.21 & 0.23 & 88.25 & 0.63 & 0.001 \\ 0.49 & 0.32 & 0.22 & 0.24 & 89.65 & 0.38 & -0.004 \\ 0.24 & 0.18 & 0.13 & 0.14 & 91.65 & 0.50 & 0.004 \\ 0.25 & 0.18 & 0.14 & 0.15 & 93.00 & 0.47 & 0.006 \\ 0.24 & 0.19 & 0.15 & 0.15 & 96.00 & 0.38 & 0.009 \\ 0.25 & 0.20 & 0.15 & 0.16 & 100.00 & 0.24 & 0.010\end{array}$

Case R2230: $\quad T=1.00$

$h_{\text {toe }}=0.58 ; x_{\text {toe }}=89.60 ; h_{c}=0.23 ; x_{c}=90.66 ;$

$h_{\mathrm{t}}=0.52 ; \mathrm{X}_{\mathrm{t}}=91.30 ; \beta_{1}=10 ; \beta_{3}=20$.

\begin{tabular}{rcccccr} 
Hmax & Hs & Hmean & Hrms & $\mathrm{X}$ & $\mathrm{h}$ & \multicolumn{1}{c}{$\eta$} \\
0.53 & 0.38 & 0.25 & 0.27 & 30.00 & 1.25 & -0.003 \\
0.42 & 0.33 & 0.22 & 0.24 & 88.10 & 0.63 & 0.002 \\
0.41 & 0.30 & 0.20 & 0.22 & 88.60 & 0.62 & -0.001 \\
0.43 & 0.33 & 0.23 & 0.25 & 90.10 & 0.38 & -0.003 \\
0.25 & 0.18 & 0.13 & 0.14 & 91.65 & 0.50 & 0.002 \\
0.25 & 0.19 & 0.14 & 0.15 & 93.00 & 0.47 & 0.005 \\
0.25 & 0.20 & 0.15 & 0.16 & 96.00 & 0.38 & 0.008 \\
0.27 & 0.20 & 0.16 & 0.17 & 100.00 & 0.24 & 0.009
\end{tabular}


Case R6210: $T=1.50$

$\mathrm{h}_{\text {toe }}=0.88 ; \mathrm{x}_{\text {toe }}=80.10 ; \mathrm{h}_{\mathrm{c}}=0.32 ; \mathrm{x}_{\mathrm{c}}=85.75 ;$

$\mathrm{h}_{\mathrm{t}}=0.66 ; \mathrm{x}_{\mathrm{t}}=86.50 ; \beta_{1}=5 ; \beta_{3}=20$.

$\begin{array}{lcccccc}\text { Hmax } & \text { Hs } & \text { Hmean } & \text { Hrms } & \mathrm{X} & \mathrm{h} & \eta \\ 0.70 & 0.46 & 0.45 & 0.35 & 30.00 & 1.25 & -0.009 \\ 0.62 & 0.45 & 0.31 & 0.34 & 79.10 & 0.94 & -0.005 \\ 0.60 & 0.45 & 0.31 & 0.34 & 79.90 & 0.88 & -0.006 \\ 0.52 & 0.41 & 0.30 & 0.32 & 84.50 & 0.42 & -0.009 \\ 0.34 & 0.23 & 0.16 & 0.17 & 87.25 & 0.64 & 0.010 \\ 0.34 & 0.26 & 0.19 & 0.20 & 91.00 & 0.52 & 0.014 \\ 0.32 & 0.26 & 0.20 & 0.21 & 95.00 & 0.41 & 0.014 \\ 0.31 & 0.23 & 0.16 & 0.18 & 100.00 & 0.23 & 0.016\end{array}$

Case R6220: $T=1.50$

$\mathrm{h}_{\text {toe }}=0.78 ; \mathrm{X}_{\text {toe }}=83.45 ; \mathrm{h}_{\mathrm{c}}=0.32 ; \mathrm{X}_{\mathrm{c}}=85.62 ;$

$h_{\mathrm{t}}=0.67 ; \mathrm{x}_{\mathrm{t}}=86.41 ; \beta_{1}=10 ; \beta_{3}=20$.

$\begin{array}{lcccccc}\text { Hmax } & \text { Hs } & \text { Hmean } & \text { Hrms } & \mathrm{X} & \mathrm{h} & \eta \\ 0.73 & 0.47 & 0.33 & 0.35 & 30.00 & 1.25 & -0.008 \\ 0.59 & 0.45 & 0.32 & 0.34 & 82.40 & 0.81 & -0.006 \\ 0.62 & 0.44 & 0.30 & 0.33 & 83.25 & 0.79 & -0.007 \\ 0.58 & 0.48 & 0.35 & 0.38 & 84.85 & 0.47 & -0.011 \\ 0.37 & 0.27 & 0.19 & 0.21 & 87.25 & 0.65 & 0.007 \\ 0.40 & 0.29 & 0.22 & 0.23 & 91.00 & 0.52 & 0.013 \\ 0.33 & 0.27 & 0.21 & 0.22 & 95.00 & 0.41 & 0.014 \\ 0.34 & 0.21 & 0.16 & 0.17 & 100.00 & 0.23 & 0.020\end{array}$

Case R6230: $T=1.50$

$\mathrm{h}_{\text {toe }}=0.79 ; \mathrm{X}_{\text {toe }}=83.51 ; \mathrm{h}_{\mathrm{c}}=0.32 ; \mathrm{X}_{\mathrm{c}}=84.83 ;$

$h_{\mathrm{t}}=0.70 ; \mathrm{x}_{\mathrm{t}}=85.62 ; \beta_{1}=15 ; \beta_{3}=20$.

$\begin{array}{ccccccc}\text { Hmax } & \text { Hs } & \text { Hmean } & \text { Hrms } & \text { X } & \text { h } & \eta \\ 0.72 & 0.48 & 0.33 & 0.36 & 30.00 & 1.25 & -0.006 \\ 0.58 & 0.42 & 0.30 & 0.32 & 82.10 & 0.82 & -0.007 \\ 0.59 & 0.44 & 0.30 & 0.33 & 83.00 & 0.80 & -0.007 \\ 0.67 & 0.52 & 0.37 & 0.40 & 84.35 & 0.46 & -0.015 \\ 0.36 & 0.27 & 0.20 & 0.21 & 87.25 & 0.65 & 0.006 \\ 0.43 & 0.30 & 0.23 & 0.24 & 91.00 & 0.53 & 0.010 \\ 0.34 & 0.27 & 0.22 & 0.23 & 95.00 & 0.41 & 0.012 \\ 0.29 & 0.20 & 0.15 & 0.16 & 100.00 & 0.23 & 0.019\end{array}$


Case R8210: $\quad T=1.75$

$h_{\text {toe }}=0.86 ; x_{\text {toe }}=80.96 ; h_{c}=0.32 ; x_{c}=86.20 ;$

$h_{t}=0.65 ; x_{t}=86.98 ; \beta_{1}=5 ; \beta_{3}=20$.

$\begin{array}{lcccccc}\text { Hmax } & \text { Hs } & \text { Hmean } & \text { Hrms } & \mathrm{X} & \mathrm{h} & \eta \\ 0.66 & 0.45 & 0.29 & 0.33 & 30.00 & 1.25 & -0.006 \\ 0.63 & 0.44 & 0.30 & 0.33 & 79.70 & 0.90 & -0.006 \\ 0.65 & 0.45 & 0.30 & 0.33 & 80.70 & 0.86 & -0.005 \\ 0.63 & 0.44 & 0.30 & 0.33 & 85.00 & 0.44 & -0.010 \\ 0.36 & 0.24 & 0.17 & 0.18 & 88.00 & 0.63 & 0.008 \\ 0.41 & 0.28 & 0.20 & 0.21 & 91.00 & 0.53 & 0.012 \\ 0.35 & 0.27 & 0.20 & 0.21 & 95.00 & 0.41 & 0.012 \\ 0.38 & 0.23 & 0.16 & 0.17 & 100.00 & 0.24 & 0.014\end{array}$

Case R8220: $\quad T=1.75$

$h_{\text {toe }}=0.72 ; x_{\text {toe }}=83.85 ; h_{c}=0.32 ; x_{c}=85.83 ;$

$h_{t}=0.66 ; X_{t}=86.57 ; \beta_{1}=10 ; \beta_{3}=20$.

$\begin{array}{lcccccc}\text { Hmax } & \text { Hs } & \text { Hmean } & \text { Hrms } & \text { X } & \text { h } & \eta \\ 0.66 & 0.44 & 0.29 & 0.32 & 30.00 & 1.25 & -0.006 \\ 0.58 & 0.42 & 0.28 & 0.31 & 82.75 & 0.81 & -0.006 \\ 0.65 & 0.45 & 0.30 & 0.33 & 83.75 & 0.73 & -0.007 \\ 0.62 & 0.47 & 0.33 & 0.36 & 85.10 & 0.47 & -0.012 \\ 0.39 & 0.27 & 0.19 & 0.20 & 88.00 & 0.63 & 0.005 \\ 0.44 & 0.30 & 0.22 & 0.24 & 91.00 & 0.54 & 0.010 \\ 0.34 & 0.27 & 0.21 & 0.22 & 95.00 & 0.41 & 0.011 \\ 0.35 & 0.22 & 0.15 & 0.17 & 100.00 & 0.24 & 0.018\end{array}$

Case R8230: $\quad \mathrm{T}=1.75$

$\mathrm{h}_{\text {toe }}=0.70 ; \mathrm{x}_{\text {toe }}=85.57 ; \mathrm{h}_{\mathrm{c}}=0.32 ; \mathrm{X}_{\mathrm{c}}=86.80 ;$

$h_{\mathrm{t}}=0.64 ; \mathrm{X}_{\mathrm{t}}=87.55 ; \beta_{1}=15 ; \beta_{3}=30$.

$\begin{array}{lcccccc}\text { Hmax } & \text { Hs } & \text { Hmean } & \text { Hrms } & \mathrm{X} & \mathrm{h} & \eta \\ 0.66 & 0.44 & 0.29 & 0.32 & 30.00 & 1.25 & -0.007 \\ 0.60 & 0.42 & 0.29 & 0.31 & 84.15 & 0.76 & -0.005 \\ 0.58 & 0.45 & 0.30 & 0.33 & 85.15 & 0.73 & -0.005 \\ 0.64 & 0.48 & 0.33 & 0.36 & 86.25 & 0.46 & -0.012 \\ 0.41 & 0.28 & 0.20 & 0.21 & 88.50 & 0.62 & 0.002 \\ 0.41 & 0.30 & 0.23 & 0.24 & 91.00 & 0.53 & 0.007 \\ 0.33 & 0.27 & 0.21 & 0.22 & 95.00 & 0.41 & 0.009 \\ 0.34 & 0.21 & 0.15 & 0.16 & 100.00 & 0.24 & 0.183\end{array}$


PLANE SLOPE IRREGULAR TESTS

Case R2000: $\quad T=1.00$

$\mathrm{m}=0.033$

\begin{tabular}{lcccccc} 
Hmax & Hs & Hmean & Hrms & $\mathrm{X}$ & $\mathrm{h}$ & \multicolumn{1}{c}{$\eta$} \\
0.48 & 0.38 & 0.25 & 0.28 & 30.00 & 1.25 & -0.001 \\
0.43 & 0.32 & 0.21 & 0.23 & 84.50 & 0.75 & 0.000 \\
0.43 & 0.31 & 0.21 & 0.23 & 85.00 & 0.73 & -0.003 \\
0.41 & 0.31 & 0.21 & 0.23 & 88.00 & 0.63 & -0.003 \\
0.34 & 0.26 & 0.18 & 0.20 & 91.00 & 0.53 & -0.003 \\
0.37 & 0.28 & 0.20 & 0.21 & 93.00 & 0.46 & -0.002 \\
0.30 & 0.24 & 0.18 & 0.19 & 96.00 & 0.33 & 0.000 \\
0.24 & 0.18 & 0.14 & 0.15 & 100.00 & 0.24 & 0.008
\end{tabular}

Case R6000: $\quad T=1.50$

$\mathrm{m}=0.033$

$\begin{array}{ccccccc}\text { Hmax } & \text { Hs } & \text { Hmean } & \text { Hrms } & \mathrm{X} & \mathrm{h} & \eta \\ 0.69 & 0.43 & 0.33 & 0.35 & 30.00 & 1.25 & -0.004 \\ 0.59 & 0.45 & 0.32 & 0.34 & 79.10 & 0.94 & -0.002 \\ 0.60 & 0.45 & 0.31 & 0.34 & 79.90 & 0.90 & -0.004 \\ 0.56 & 0.45 & 0.31 & 0.34 & 84.50 & 0.75 & -0.006 \\ 0.48 & 0.37 & 0.27 & 0.29 & 87.25 & 0.65 & -0.006 \\ 0.49 & 0.37 & 0.27 & 0.29 & 91.00 & 0.53 & -0.004 \\ 0.35 & 0.28 & 0.22 & 0.23 & 95.00 & 0.41 & 0.004 \\ 0.36 & 0.20 & 0.15 & 0.16 & 100.00 & 0.23 & 0.019\end{array}$

Case R8000: $\quad T=1.75$

$\mathrm{m}=0.033$

$\begin{array}{lcccccc}\text { Hmax } & \text { Hs } & \text { Hmean } & \text { Hrms } & \mathrm{X} & \mathrm{h} & \eta \\ 0.68 & 0.45 & 0.30 & 0.33 & 30.00 & 1.25 & -0.004 \\ 0.62 & 0.45 & 0.30 & 0.33 & 79.70 & 0.90 & -0.005 \\ 0.64 & 0.45 & 0.30 & 0.33 & 80.70 & 0.86 & -0.007 \\ 0.59 & 0.45 & 0.30 & 0.33 & 85.00 & 0.73 & -0.007 \\ 0.49 & 0.37 & 0.26 & 0.28 & 88.00 & 0.63 & -0.006 \\ 0.49 & 0.38 & 0.27 & 0.29 & 91.00 & 0.53 & -0.005 \\ 0.37 & 0.30 & 0.22 & 0.23 & 95.00 & 0.41 & 0.002 \\ 0.35 & 0.22 & 0.14 & 0.16 & 100.00 & 0.23 & 0.016\end{array}$




$$
a(m)
$$

A

$A_{b}$

$A_{s}$

$A_{v}$

$\mathrm{b}(\mathrm{m})$

B

C

C

$\mathrm{C}_{1}$

$\mathrm{C}_{2}$

$\mathrm{C}_{\mathrm{g}}$

$\mathrm{C}_{\mathrm{gb}}^{\prime}$

$\mathrm{C}(\mathrm{m})$

$C\left(B_{1}\right)$

$\mathrm{D}_{1}$

$\mathrm{D}_{2}$

E

$\mathrm{f}_{\mathrm{p}}$

$F$

$\mathrm{f}(\mathrm{m})$

$\mathrm{F}(\mathrm{m})$

$\mathrm{F}_{\mathrm{s}}$

$g$

G

$\mathrm{G}(\mathrm{m})$

$\mathrm{h}$

h'

$\mathrm{h}_{\mathrm{b}}$

$h_{c}$
Empirical coefficient in breaker depth equation, Weggel $(1972)^{*}$ and present study.

Coefficient in breaker height equation (Goda 1975)

Cross-sectional area of reef

Cross-sectional area of surface roller

Cross-sectional area of vortex

Empirical coefficient in breaker depth equation, Weggel (1972) and present study.

Nondimensional energy flux

Coefficient in equation for depth over bar crest

Wave celerity

Coefficient in generic breaker index equations

Coefficient in generic breaker index equations

Group speed of waves

Group speed of waves at breaking in the presence of return flow

Empirical coefficient in breaker height equation

Empirical exponent in breaker height equation for barred profiles

Empirical function for breaker height (Weggel 1972)

Empirical function for breaker height (Weggel 1972)

Wave energy

Spectral peak frequency

Wave energy flux

Function of beach slope

Empirical function in breaker height (Weggel 1972)

Stable wave energy flux

Acceleration due to gravity

Spectral peak enhancement parameter

Empirical function in breaker height (Weggel 1972)

Still-water depth

Water depth in horizontal section of wave tank

Water depth at breaking

Water depth at crest of bar

* See References at the end of the main text. 


\begin{tabular}{|c|c|}
\hline$h_{s}$ & Water depth at reef toe \\
\hline$h_{\mathrm{t}}$ & Water depth at trough of bar \\
\hline $\mathrm{H}$ & Wave height \\
\hline $\mathrm{H}^{\prime}$ & Wave height measured in horizontal section of wave tank \\
\hline $\mathrm{H}_{1 / 3}$ & $\begin{array}{l}\text { Average of the highest one-third wave heights in a } \\
\text { wave record }\end{array}$ \\
\hline $\mathrm{H}_{\mathrm{b}}$ & Breaking wave height \\
\hline $\mathrm{H}_{\mathrm{b}}^{\prime}$ & Breaking wave height in the presence of return flow \\
\hline $\mathrm{H}_{\mathbf{1}}$ & Incident wave height \\
\hline $\mathrm{H}_{\max }$ & Maximum wave height \\
\hline $\mathrm{H}_{\mathrm{o}}$ & Deepwater wave height \\
\hline$\left(\mathrm{H}_{\mathrm{o}}\right)_{\mathrm{m}}$ & Wave height in model \\
\hline$\left(\mathrm{H}_{\mathrm{o}}\right)_{\mathrm{pr}}$ & Wave height in prototype. \\
\hline$\left(\mathrm{H}_{\mathrm{s}}\right)_{0}$ & Significant deepwater wave height \\
\hline $\mathrm{H}_{\mathrm{r}}$ & Reflected wave height \\
\hline $\mathrm{H}_{\mathrm{rms}}$ & Root-mean-square wave height \\
\hline $\mathrm{H}_{\mathbf{s}}$ & Significant wave height \\
\hline $\mathrm{H}_{\mathrm{s}}^{\prime}$ & $\begin{array}{l}\text { Significant wave height measured in horizontal section of } \\
\text { wave tank }\end{array}$ \\
\hline $\mathrm{H}_{0} / \mathrm{L}_{\mathrm{o}}$ & Deepwater wave steepness \\
\hline$\left(\mathrm{H}_{\mathrm{o}} / \mathrm{L}_{\mathrm{o}}\right)_{\mathrm{cr}}$ & Critical wave steepness for reflection (Miche 1951) \\
\hline$\left(\mathrm{H}_{\mathrm{o}} / \mathrm{L}_{\mathrm{o}}\right)_{\mathrm{m}}$ & Model deepwater wave steepness \\
\hline$\left(\mathrm{H}_{\mathrm{o}} / \mathrm{L}_{\mathrm{o}}\right)_{\mathrm{pr}}$ & Prototype deepwater wave steepness \\
\hline $\mathrm{k}$ & Wave number \\
\hline $\mathrm{K}$ & Coefficient in breaker height equation (Goda 1975) \\
\hline $\mathrm{K}$ & $\begin{array}{l}\text { Dimensionless decay coefficient (Dally, Dean, and Dalrymple } \\
\text { 1985a, 1985b) }\end{array}$ \\
\hline $\mathrm{K}_{\mathrm{r}}$ & Reflection coefficient \\
\hline $\mathrm{L}$ & Wavelength \\
\hline$L^{\prime}$ & Wavelength calculated in horizontal section of wave tank \\
\hline $\mathrm{L}_{\mathrm{b}}$ & Wavelength at break point \\
\hline $\mathrm{L}_{\mathrm{o}}$ & Deepwater wavelength \\
\hline$\left(\mathrm{L}_{\mathrm{s}}\right)_{0}$ & Significant deepwater wavelength \\
\hline$\left(L_{p}\right)_{0}$ & Peak deepwater wavelength \\
\hline $\mathrm{L}_{\mathrm{s}}$ & Wavelength at reef toe \\
\hline $\mathrm{m}$ & Beach slope \\
\hline $\mathrm{n}$ & Ratio of group velocity to individual wave velocity \\
\hline
\end{tabular}


Empirical exponent wave decay equation (Smith and Kraus 1988)

Exponent in generic breaker index equations

Exponent in generic breaker index equations

Empirical exponent in breaker height equation for plane slopes

Empirical exponent in breaker height equation for barred profiles

Reef reflection parameter

Runup

Average runup

Two-percent runup

Significant runup

Exponent in breaker height equation (Goda 1975)

Horizontal distance from the bar crest to the seaward toe of the bar

Radiation stress

Wave period

Wave period in model

Peak wave period

Wave period in prototype

Significant wave period

Horizontal velocity component

Horizontal velocity component at break point

Horizontal distance

Coefficient in breaker height equation (Goda 1975)

Plunge distance

Splash distance

Penetration distance

Reef height

Coefficient in hydraulic jump dissipation rate equation

Beach slope angle

Lower (primary) seaward bar angle

Upper (secondary) seaward bar angle

Shoreward bar angle

Empirical stable wave factor equal to ratio of stable wave height to water depth

Ratio of wave height to water depth at breaking

Energy dissipation rate 
Free-surface water elevation

Surf similarity parameter

offshore surf similarity parameter

Inshore surf similarity parameter

Water density

Spectral width parameter

Low-frequency spectral width parameter

High-frequency spectral width parameter

Ratio of breaking wave height to deepwater wave height 




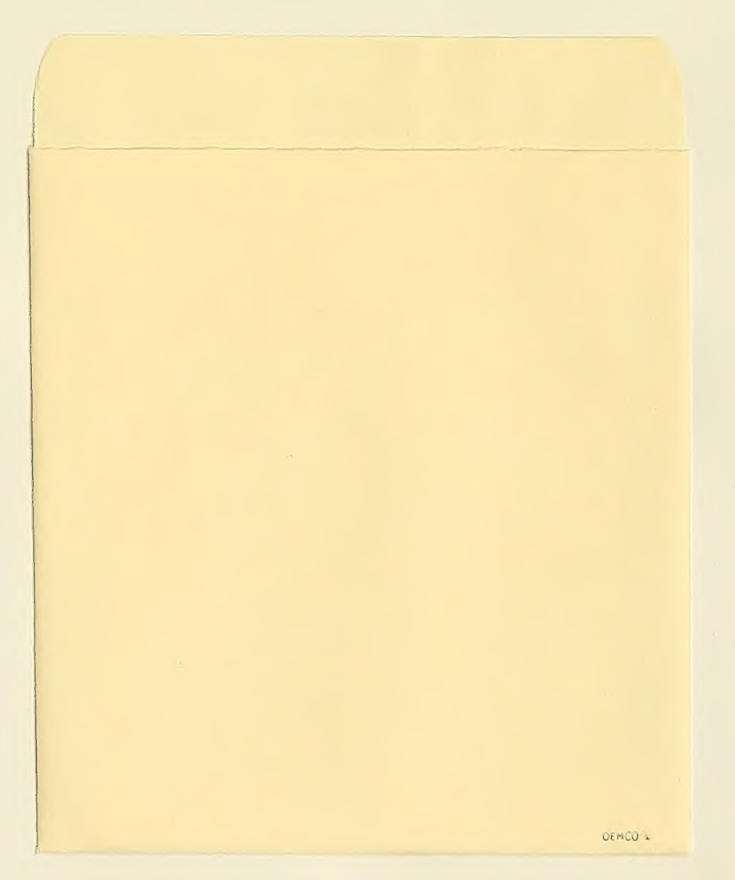




$$
\text { - }
$$

